\title{
The Application of Ecological Resilience to Urban Landscapes
}

By

Emilio Jose Garcia

\begin{abstract}
A thesis
submitted to the Victoria University of Wellington

in fulfilment of the requirements for the degree of

Doctor of Philosophy

in Architecture
\end{abstract}

Victoria University of Wellington

2013 


\begin{abstract}
The understanding of resilience is a key concept for improving the adaptive capacity of cities to deal with and take benefits from unpredictable changes while keeping on evolving. However, in urbanism and architecture, a theoretical framework to use resilience in urban, landscape and architectural design is still needed. This thesis proposes an instrumental theory that can work as a link between the ecological theory of resilience and its utilisation in urban and architectural design. Through the implementation of this new knowledge, it should be possible to provide insight into the structures, dynamics and selforganizing processes that sustain the resilience capacity of cities. The research has developed a methodology for analysing the ecological resilience of urban landscapes using an urban morphological approach. The method has been tested on case studies in Auckland, New Zealand, and Tokyo, Japan, showing it is possible to observe changes in the resilience of the urban fabric.
\end{abstract}




\section{Table of Contents}

The Application of Ecological Resilience to Urban Landscapes ....................... 1

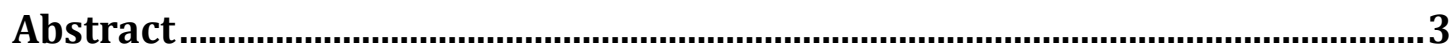

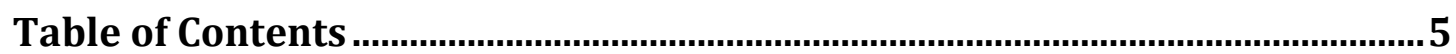

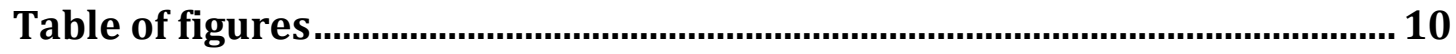

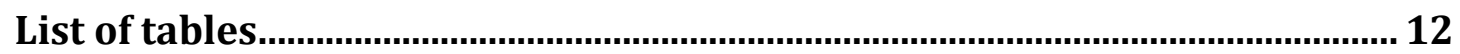

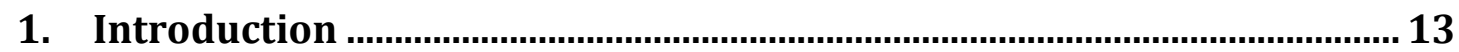

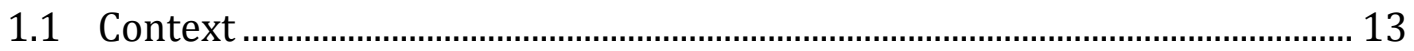

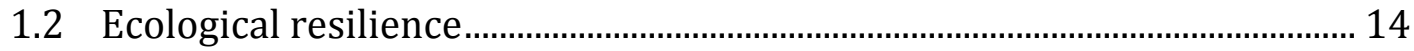

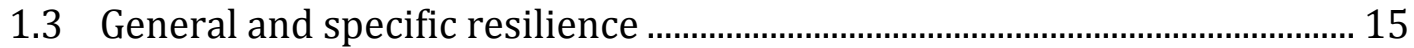

1.4 Ecological resilience and socio-ecological systems ...................................... 15

1.5 From socio-ecological systems to socio-ecological landscapes ................... 16

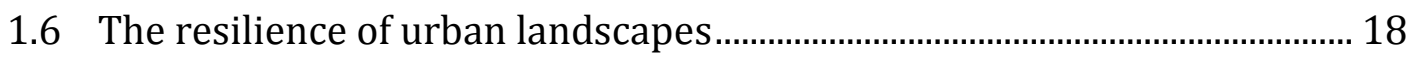

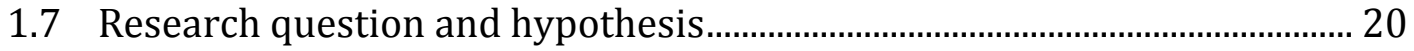

1.8 Purpose and general objectives of the research .............................................. 22

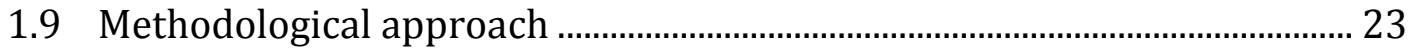

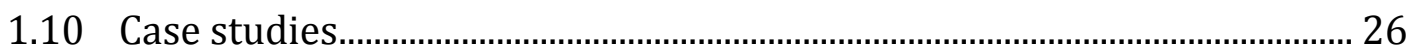

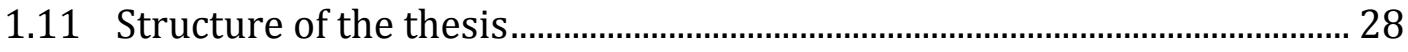

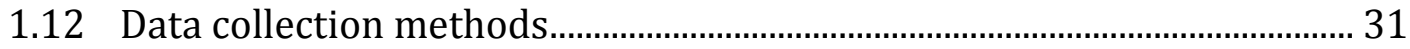

2. Ontology: Ecological resilience, philosophy and urban morphology.... 33

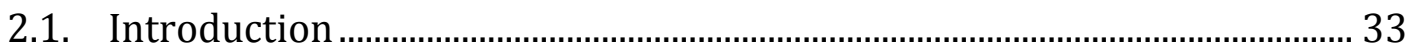

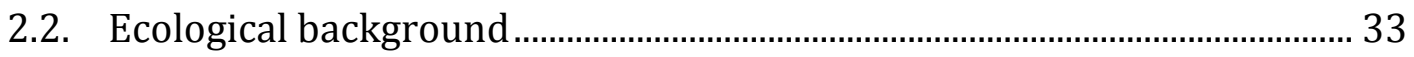

2.2.1 The behaviour of complex adaptive systems …….................................... 34

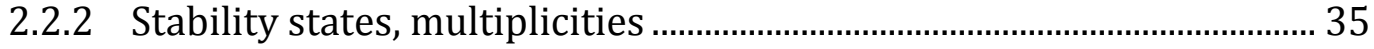

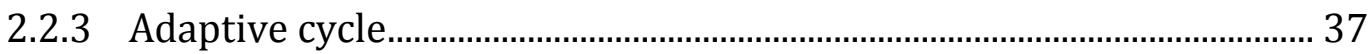

2.2.4 Panarchy: change within and across scales ............................................. 39

2.2.5 The Textural-Discontinuity Hypothesis in ecological resilience ......... 40

2.3. Philosophical approach ................................................................................ 43

2.3.1 A complex approach to the identity of an urban landscape: assemblages and multiplicities................................................................................ 45

2.3.2 Multiple stability states and identities................................................... 47 
2.3.3 Multiples identities assembled in a Panarchy

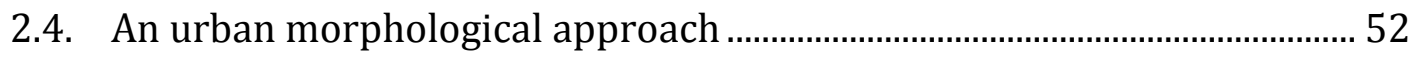

2.4.1 Adaptive cycles in urban landscapes: the burgage cycle ........................ 53

2.4.2 Textural-Discontinuity Hypothesis in urban landscapes ....................... 55

2.4.3 Aggregations and discontinuities in urban landscapes.......................... 58

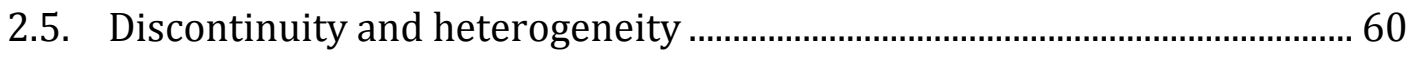

2.5.1 Discontinuities and public-private interfaces........................................ 61

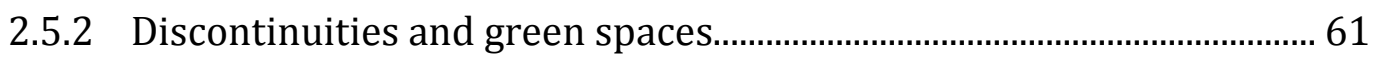

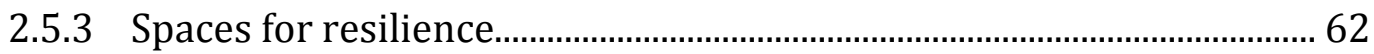

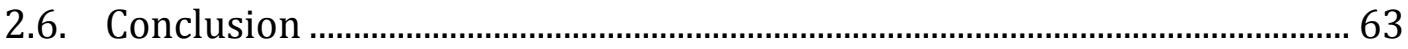

3. Criteria for assessing the ecological resilience of urban landscapes.... 67

3.1 Introduction

3.2 Assessing the dynamics of change in analysing the ecological resilience of urban landscapes 68

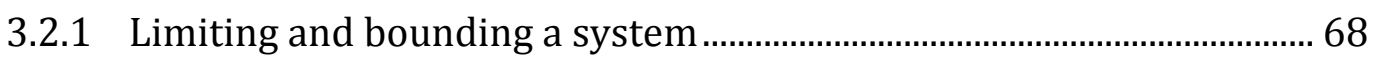

3.2.2 What are the main issues affecting the system? .................................... 69

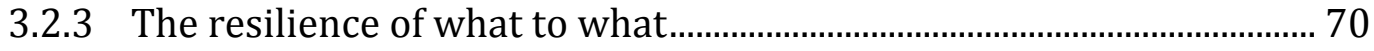

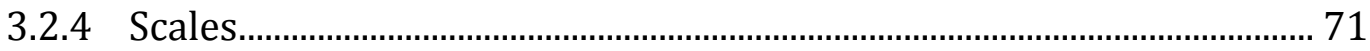

3.2.5 Timeline: assessing change in urban landscapes by identifying cycles

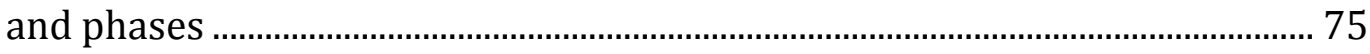

3.2.6 Constructing and evaluating an urban Panarchy....................................... 79

3.3 Assessing resilience through the analysis of discontinuities: the urban

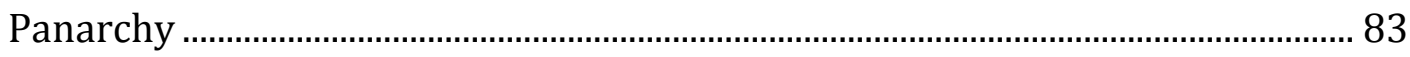

3.4 The use of Panarchy and discontinuities in urban studies ........................... 84

3.5 Finding aggregations and discontinuities in urban landscapes................... 86

3.6 The use of aggregations and discontinuities to assess resilience in urban

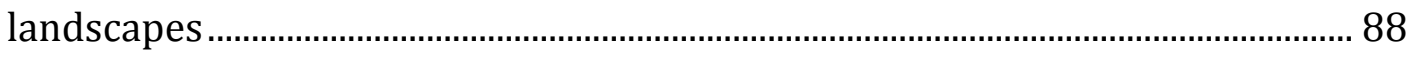

\section{Assessing resilience and the dynamics of change in the urban}

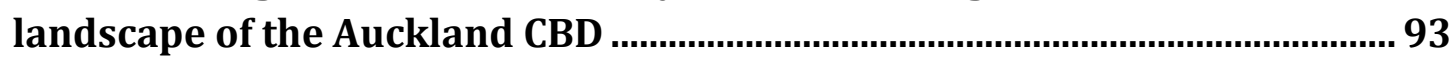

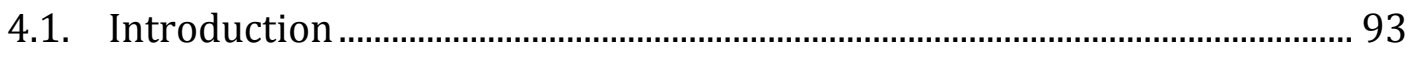

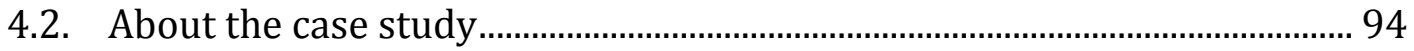

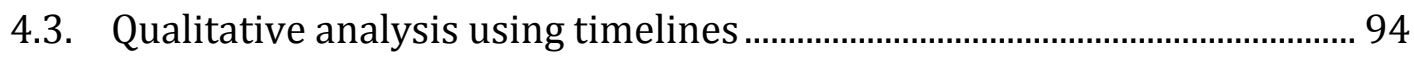

4.3.1 Timeline at the scale of New Zealand........................................................ 96

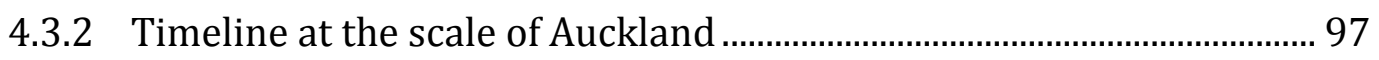


4.3.3 Synthesis of timelines: morphological changes in the urban landscape

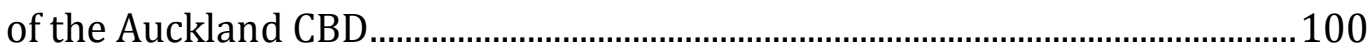

4.3.4 Observations about the qualitative approach to timelines..................103

4.4. Quantitative analysis using timelines ............................................................103

4.4.1 General Observations about change in BF, PL, BL and ST ...................105

4.4.2 Quantity of change in space: percentage of change of BF, PL, BL, and ST 107

4.4.3 Quantity of change in time: average rate of change of BF, PL, BL and ST 110

4.4.4 Synthesis of the quantity and speed of change

4.5. Conclusion

5. Resilient assemblages: identity and resilience

5.1 Introduction

5.2 Structure of the chapter

5.3 Theoretical framework: resilient assemblages

5.4 Nezu and its identity.

5.5 Study 1: the role of the structure of urban landscapes in the resilience capacity of Nezu

5.5.1 Method for measuring morphological change in the urban landscape of Nezu

5.5.2 Results from the analysis of morphological changes in sizes of blocks, streets and plots

5.5.3 Results from the analysis of morphological transformations related with degree of change

5.6 Study 2: the role of interfaces in the resilience capacity of the built environment of Nezu

5.6.1 Method to finding discontinuities and aggregations through cluster analysis.

5.7 Conclusions

6. Assessing and measuring resilience

6.1 Introduction 145

6.2 Theoretical framework: Panarchy and the TDH 146

6.3 Case studies

6.4 Methods

6.5 Qualitative analysis 
6.6.1 Analysis of heterogeneity within scale ................................................158

6.6.2 Analysis of heterogeneity across scales ..................................................160

5.6.2 Evolution of the heterogeneity of Nezu and Auckland GS and BE ....161

6.6.3 Difference in change in the heterogeneity of Nezu and Auckland with and without Green Space

6.6.4 Rate of change in the heterogeneity of Nezu and Auckland with and without Green Spaces.

6.6.5 Synthesis of the evolution of the heterogeneity of the urban landscapes of Nezu and Auckland

6.7 Results

6.8 Conclusion

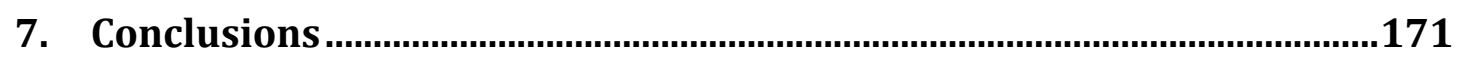

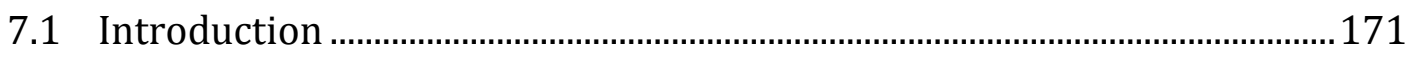

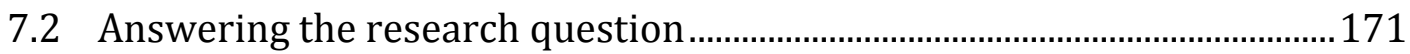

7.3 Theoretical evidence: thinking about how to apply ecological resilience to the analysis of urban landscapes.

7.4 Practical evidence: ways of applying ecological resilience to the analysis of urban landscapes.

7.4.1 Ecological resilience can be applied to the analysis of urban landscapes by assessing the dynamics of change in key elements of the built environment

7.4.2 Ecological resilience can be applied to the analysis of urban landscapes by comparing changes in key elements of the built environment against persistence or shifts in the identity of place.

7.4.3 Ecological resilience can be applied to the analysis of urban landscapes by measuring changes in the heterogeneity of the built environment of urban landscapes

7.5 Weaknesses 180

7.6 Discussion and opportunities for further research 181

8. Bibliography 185

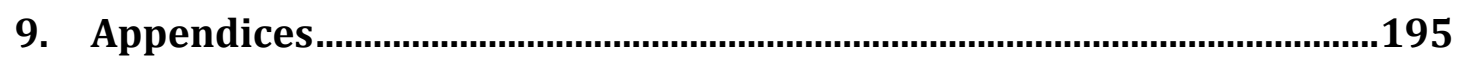

Appendix 1: Timeline for Auckland (chapter 4) ........................................................196

Appendix 2: Maps of blocks in the urban landscape of Auckland CBD..............198

Appendix 3: Maps of plots in the urban landscape of Auckland CBD. 199 
Appendix 4: Maps of streets in the urban landscape of Auckland CBD.............200 Appendix 5: Maps of building footprints in Auckland CBD (chapter 4)............201 Appendix 6: areas and number of features per BF, PL, BL, ST (chapter 4)......202 Appendix 7: Key map with name of elements of the built environment (chapter 5) 203

1. Appendix 8: Degree of change in blocks in Nezu (chapter 5).......................204

2. Appendix 9: Degree of change in plots in Nezu (chapter 5) ..........................205

3. Appendix 10: Degree of change in streets in Nezu (chapter5) ....................206

4. Appendix 11: Areas and clusters in Nezu 1888 (chapter 5 and 6)..............207

5. Appendix 12: Areas and clusters in Nezu 1919 (chapter 5 and 6)..............208

6. Appendix 13: Areas and clusters in Nezu 1932 (chapter 5 and 6)..............209

7. Appendix 14: Areas and clusters in Nezu 1956 (chapter 5 and 6)..............210

8. Appendix 15: BF Areas and clusters in Nezu 2003 (chapter 5 and 6) .......211

9. Appendix 16: Areas and clusters in Nezu 2003 (chapter 5 and 6)..............212

10. Appendix 17: Areas and clusters in Auckland 1882 (chapter 6)...............213

11. Appendix 18: Areas and clusters in Auckland 1908 (chapter 6)...............214

12. Appendix 19: Areas and clusters in Auckland 1931 (chapter 6)...............215

13. Appendix 20: Areas and clusters in Auckland 1966 (chapter 6)...............216

14. Appendix 21: Areas and clusters in Auckland 2008 (chapter 6)...............217 


\section{Table of figures}

Figure 1-1 Socio-ecological systems as socio-ecological landscapes ...................... 17

Figure 1-2. A complex approach to the research .............................................26

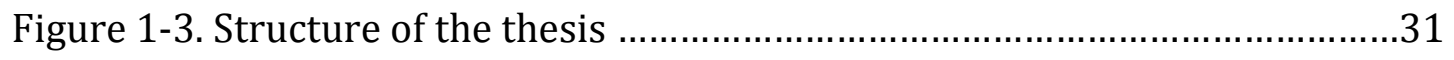

Figure 2-1. The multiple stability states of a landscape based on (Folke et al., 2004) 36

Figure 2-2. Multiple stability states and cross scales dynamics based on (Folke et al., 2004) 37

Figure 2-3. The adaptive cycle based on (L. H. Gunderson \& Holling, 2002) ........ 38

Figure 2-4. A Panarchy based on (L. H. Gunderson \& Holling, 2002) ........................ 40

Figure 2-5. The Textural-Discontinuity Hypothesis in ecosystems ........................ 42

Figure 2-6. Dimensions implied in the proposal for an assembled identity .......... 44

Figure 2-7. Becoming an assembled identity .................................................................. 46

Figure 2-8. Multiplicities and identity …………............................................................ 48

Figure 2-9. Multiplicities and multiple identities across scales................................. 49

Figure 2-10. Multiples identities assembled in a Panarchy....................................... 51

Figure 2-11. Applying the adaptive cycle to the burgage cycle................................. 54

Figure 2-12. Adaptive cycle in the identity of place ..................................................... 55

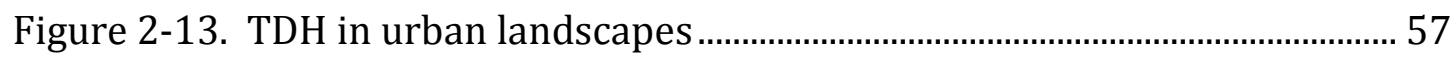

Figure 2-14. Aggregations and discontinuities in urban landscapes....................... 59

Figure 3-1. Scale dimensions based on (Gibson et al., 2000; Wu \& Li, 2006) ....... 72

Figure 3-2. Diagram of the cost-benefits of complexity (Tainter, 2000). B1, B2, C1,

C2, C3 are only references to imply different amounts of complexity and

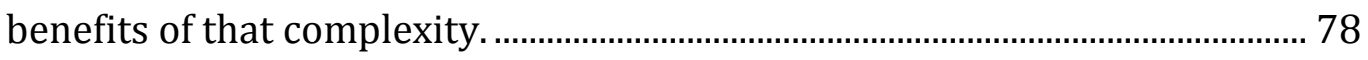

Figure 4-1. Multiple historical phases at different scales..........................................101

Figure 4-2. Timeline..................................................................................................102

Figure 4-3. Evolution of total areas (sum) of BF, PL, BL..........................................106

Figure 4-4. Evolution of number of features per BF, PL, BL, ST..............................106

Figure 4-5. Percentage of change in the sum of BF, PL, BL, ST .................................108

Figure 4-6. Percentage of change in the number of BF, PL, BL, ST .........................108

Figure 4-7. Percentage of change in the mean of BF, PL, BL, ST ..............................109 
Figure 4-8. Sum average rate of change

Figure 4-9. Number of features average rate of change

Figure 4-10. Mean average rate of change

Figure 5-1. Map of Tokyo and Nezu (Muminovic et al., 2012a)

Figure 5-2. Major earthquakes and fires in Tokyo from Edo to Showa periods (1600 to 1945) (Muminovic et al., 2012a)

Figure 5-3. Map of burned areas in 1923 (left) and 1945 (right) (Anonymous, 1988; map, 1990) 125

Figure 5-4. Size classes of blocks, streets and plots (top-bottom) 130

Figure 5-5. Diversity of blocks and streets through time. 131

Figure 5-6. Average degree of change in blocks, streets and plots. 133

Figure 5-7. Intensity of change through time

Figure 5-8. Aggregations and discontinuities in the structure of ST, BL and BF138

Figure 5-9. Clusters of public private interfaces at BF, BL and ST levels 140

Figure 5-10. Clusters of public-private interfaces within and across BF, BL and ST levels

Figure 6-1. The evolution of heterogeneity in the urban landscape of Auckland 151

Figure 6-2. Structures of aggregations and discontinuities in GS, ST, BL, PL in Auckland 152

Figure 6-3. The evolution of heterogeneity in the urban landscape of Nezu ......153

Figure 6-4. Structures of aggregations and discontinuities in GS, ST, BL, PL in Nezu. 154

Figure 6-5.Comparison between the structure of aggregations and discontinuities in the GS and BE (ST, BL, PL) of Auckland and Nezu 156 


\section{List of tables}

Table 2-1. Toward a resilience assemblage approach: comparative view of urban landscapes from assemblage and resilience............................................................. 50

Table 4-3. Quantity and speed of change in BF, PL, BL, ST ...........................................113

Table 4-4. Diagnosis of quantity and speed of change ....................................................114

Table 6-2. Similarities and differences in the behaviour of GS and BE in Auckland and Nezu

Table 6-3. Analysis of the evolution of richness, evenness and diversity in Auckland. 158

Table 6-4. Analysis of the evolution of richness, evenness and diversity in Nezu.159

Table 6-5. Average performance in heterogeneity in GS, ST, PL, BL of Auckland and Nezu

Table 6-6. Heterogeneity of Nezu and Auckland BE and GS. 161

Table 6-7. Heterogeneity of Nezu BE and Auckland BE. 161

Table 6-8. Heterogeneity of Nezu GS and Auckland GS.. 161

Table 6-9. Change difference in Nezu and Auckland BE and GS. 162

Table 6-10. Change difference in Nezu and Auckland BE 162

Table 6-11. Change difference in Nezu and Auckland GS .163

Table 6-12. Rate of change in Nezu and Auckland BE and GS 164

Table 6-13. Rate of change in Nezu and Auckland BE 164

Table 6-14. Rate of change in Nezu and Auckland GS. .164

Table 6-15. Comparison of heterogeneity, rate of change and change difference between Nezu and Auckland .165 


\section{Introduction}

\subsection{Context}

In the last decades humanity has realized that the way in which the resources of the environment are treated is putting at risk the future of the present and next generations (Millennium Ecosystem, 2005; Tainter, 1995). One of the challenges that cities have to face is dealing with unpredictable changes and hazards. Some of the reasons for thinking about and designing more adaptable cities are the non-linear behaviour of an altered natural environment, an unstable cultural environment, and the challenges presented by the rapid land use change of the last few decades (C. Allen \& Holling, 2010).

Resilience studies were born in ecology as a response to a worldwide concern about how to manage resources in systems of people and nature, and especially how to deal with the unpredictable behaviour of complex adaptive systems (B. Walker \& Salt, 2006). Cities, as the built environment of socioecological systems, are also complex adaptive systems (Alberti \& Marzluff, 2004; Garmestani, C. Allen, \& Bessey, 2005; Gunderson \& Holling, 2002) that need to deal with socio-ecological threats. These crises can affect the built environment of urban landscapes producing transformations that may push the urban system to generate more or less unpredictable outcomes that increase the difficulty of managing urban environments. The understanding of resilience hence becomes a key concept for improving the adaptive capacity of the built environment of cities. However, the understanding and assessment of how the dynamics of change in the built environment of cities enhance or decrease the resilience capacity of urban landscapes, at the face of disturbances, is a subject not commonly explored in urban studies. 


\subsection{Ecological resilience}

Etymologically, resilience comes from the Latin resilio, and means to jump back (Klein, Nicholls, \& Thomalla, 2003). In ecology, resilience is understood from two viewpoints, ecological and engineering resilience (Gunderson, 2000). Engineering resilience is related with the recovery time that a system takes before coming back to its stability state (Gunderson, 2000). This position implies that systems work around a single equilibrium state that they lose or recover in more or less time. The faster a system recovers the more resilient it is (Pimm, 1984). However, scholars in ecological resilience (Gunderson, 2000; Holling, 1973; Holling, 1987; Peterson, C. Allen, \& Holling, 1998) have criticized the idea of a system working around a single equilibrium state. The proposal is that as complex adaptive systems change their equilibrium points also change (Folke et al., 2004). Therefore a resilience response to a disturbance is the capacity of the system to maintain interrelationships while absorbing change happening at different scales. Precisely, it is "the persistence of relationships within a system and is a measure of the ability of these systems to absorb changes of state variables, driving variables, and parameters, and still persist" (Holling, 1973, p. 19). From an ecological point of view, complex systems have the capability to work in multiple stability states and to absorb changes before crossing to a different regime (B. Walker \& Meyers, 2004).

Ecological resilience is based upon an evolutionary view that acknowledges that nature is evolving and adapting continuously (Gunderson \& Holling, 2002). A complex system is never in its equilibrium point, but moving in between a set of variables (Genkai-Kato, 2007). Accordingly, with a perspective of evolving nature, resilience can be comprehended as a mechanism of adaptation that complex systems have in order to take part in evolutionary processes (Kauffman, 1993; Levin, 2005). Therefore, a resilience approach is not about defining the right size, final state or best performance of a system but is about finding a way to keep the system evolving while maintaining the social and ecological systems alive. Resilience is not a state to be reached but a sense of emergence that a complex adaptive system relies on when facing a more or less demanding need for transformation. The development of a better understanding of resilience in urban landscapes could help to improve the management and 
assessment of resilience in order to generate criteria related to whether to reduce or increase the resilience capacity of a system.

\subsection{General and specific resilience}

In resilience theory, two scales of analysis have been defined: general and specific resilience (B. Walker, 2007). General resilience gives attention to the resilience of the whole system. It is focused on long terms results, and could easily be weakened if all the attention is given to responding to the resilience of small and short-term issues. Specific resilience is related to targeted alterations at precise scales. It is focused on the relationship between the state of a system and a specific disturbance, in other words the resilience of what to what (Carpenter, Walker, Anderies, \& Abel, 2001). An example would be the resilience of socio-ecological landscapes to the increasing complexity produced by an unsustainable development of socio-ecological systems.

The important point about general and specific resilience is to realize that a system can be resilient at larger scales while not being resilient at smaller scales or vice versa. In consequence the resilience of a system can be something desirable or not. For example, the eradication of violence in neighbourhoods with high rates of crime is highly resilient to strategies that try to fight against these conducts. These maladaptive processes (Gunderson \& Holling, 2002; Rappaport, 1977) show that the resilience of complex adaptive systems needs to be treated as an attribute of the system and not as a category to be reached.

\subsection{Ecological resilience and socio-ecological systems}

Ecological resilience theorists have developed a theoretical framework with which to comprehend and produce knowledge through the assessment of global changes occurring in socio-ecological systems (Gunderson, Garmestani, \& C. Allen, 2009). The integration of social and ecological systems into one dimension permits a broader approach in order to produce a better understanding of the dynamics of change happening globally (Gallopín, 2006; Holling, 2004; Redman \& Kinzig, 2003). Urban landscapes, understood as a subsystem of the man-made physical environment of a socio-ecological system, can benefit from this integrative approach because investigation into their 
dynamics of change will be linked with the state of the whole socio-ecological system.

The analysis of the resilience capacity of a socio-ecological system could produce a better understanding of the unpredictable responses that a complex adaptive system exhibits in its behaviour (Redman \& Kinzig, 2003). This is particularly important when analysing the situation and performance of a system in relationship with the plans and strategies applied for its management (Holling \& Goldberg, 1971). The ecological resilience approach can be important for learning about the long-term dynamics that can delineate the future of a system and using this knowledge for the planning and management of natural and social resources (Grimm, Pickett, \& Redman, 2000). For all these reasons, the resilience understanding of urban landscapes could be essential for providing managers and designers with information about the adaptive capacity of a socioecological system before it faces new, unpredictable changes.

\subsection{From socio-ecological systems to socio-ecological landscapes}

In order to make societies more aware of their habitats and how these are potentially adaptable to both natural and man-made hazards, a broader understanding of socio-ecological systems (Gallopín, 2006) as socio-ecological landscapes (SELs) is necessary. A SEL is the landscape produced by a socioecological system (SES) (B. Walker \& Salt, 2006). Viewing urban systems in this way is a first step in designing them to be more adaptable in the face of external and internal hazards. 


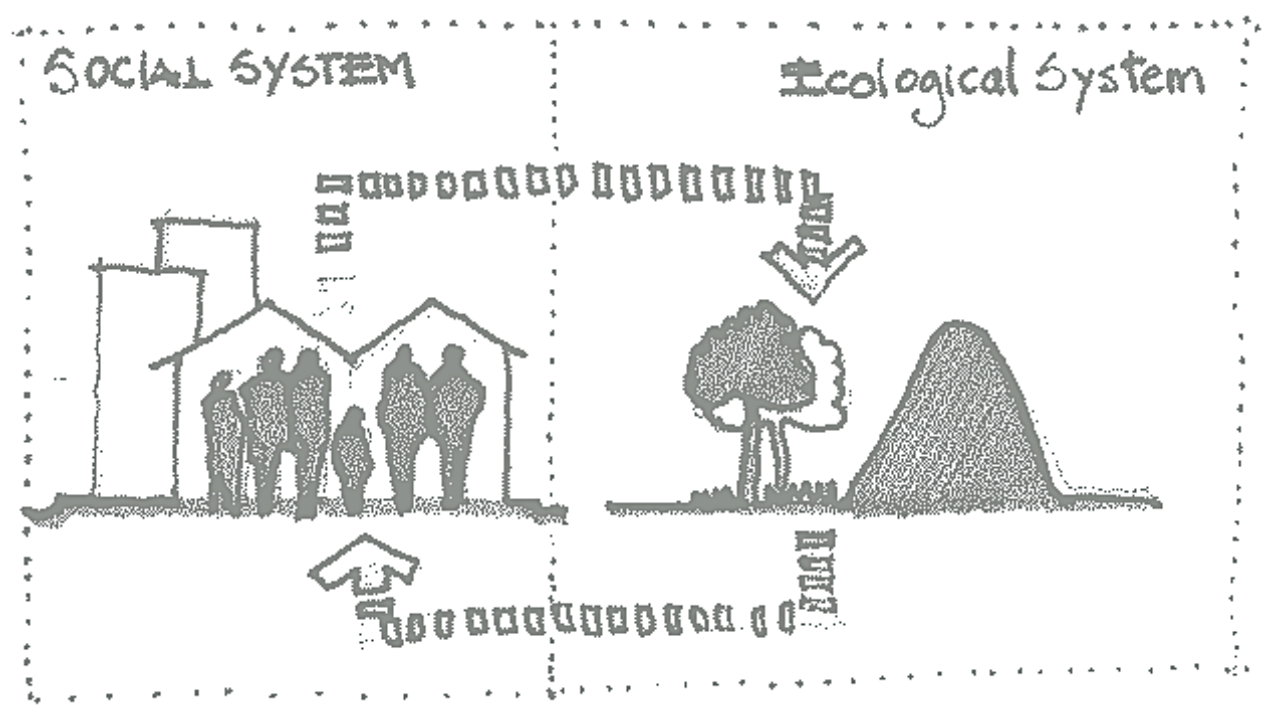

Figure 1-1 Socio-ecological systems as socio-ecological landscapes

The understanding of urban landscapes as a subsystem of a socioecological landscape implies acknowledgement of the importance of land as an ultimate resource that is impacted by the evolutionary process of transformations occurring in the built environment of urban landscapes. The importance of the use and occupation of the territory becomes a key issue for the performance, present and future, of a socio-ecological system. Urban land and space are not often understood and analysed as natural resources that deserve to be managed in a way that is similar to how water or air are treated.

The organization and occupation of land by different urban morphological patterns can imply the presence of discrete intensities that impact on the occupation and exploitation of the territory. One important non-market asset of urban space that is linked with land resources is the capacity that urban systems have to produce spaces to buffer unpredictable changes. Built form patterns when combined with different rates of change, at discrete scales, determine a level of consumption of the land that may impact on the resilience capacity of the urban landscape to deal with unpredictability. The intensity of land exploitation in the built environment of urban landscapes will, in turn, increase or lessen the resilience capacity of a city through the generation of the necessary diversity of land-related opportunities. Understanding the relationship between land 
occupation and the resilience capacity of urban landscapes may be essential for the future of cities in the face of more people and fewer resources.

\subsection{The resilience of urban landscapes}

The importance of ecological resilience as an emergent and growing field of research can also be perceived in urban studies. However the analysis of the resilience capacity of a socio-ecological landscape using the built environment of an urban landscape as the subject of analysis is not very common.

The purpose of this thesis is not to claim that the comparison between urban landscapes and ecosystems is original. The idea of comparing urban systems, cities, and particularly urban landscapes with ecological systems has been consolidated through debate about cities in landscape design, architecture and urbanism. It can be argued that at least since the Renaissance urban environments have been compared to ecosystems. From Francesco Milizia (1785) to Patrick Geddes (1915) and Lewis Mumford (Luccarelli, 1995), cities have been assumed to be natural elements and in several instances nature has been taken as inspiration for solving design issues. In this thesis, the link between urban landscapes and ecosystems is approached from a different perspective. Leaving apart dealing with the organic (nature) in a metaphorical way, this thesis sets out to link natural and social systems since both of them can be analysed as complex adaptive systems.

Despite the fact that designers in urbanism, architecture and landscape architecture work and deal everyday with the built environment, the scientific and methodological knowledge about the dynamics of change in the evolution of cities and urban landscapes remains a largely unexplored field with many opportunities for improving knowledge in this area (Batty, 2010; Wegener, Gnad, \& Vannahme, 1986). Even though important advances have been made in the application of ecological studies to urban systems (Alberti, 2009; Alberti \& Marzluff, 2004; Alberti et al., 2003; Holling \& Goldberg, 1971; Pickett et al., 1997; Pickett et al., 2008) the foundations for performing a resilience analysis in the built environment of urban landscapes are still less developed than the foundations and advances achieved in ecology (Gunderson, C. Allen, \& Holling, 2009). 
Research in ecological resilience has been focused on the impacts of social systems over natural systems, particularly the way in which social systems manage and exploit natural resources (Elmqvist et al., 2004; Folke et al., 2004). In urban studies, most of the resilience literature discusses the impact of natural hazards in urban areas and describes the engineering responses to those situations, targeting a particular aspect of a hazard and its effects, while the study of the dynamics and attributes of the affected system (human habitat) before and after the event, are easily forgotten or not taken into account (Adger, Hughes, Folke, Carpenter, \& Rockstrom, 2005; Evan \& Warren, 2004; Fleischhauer, 2008; Klein et al., 2003; McGlade, McIntosh, \& Jeffrey, 2008). A growing number of publications related with the resilience of the built environment is emerging, but most are basically focused on the responses to disaster situations (Birch \& Wachter, 2006; Chang, Wilkinson, Potangaroa, \& Seville, 2010, 2011; Haigh \& Amaratunga, 2010; L. Vale \& Campanella, 2005). This literature is more focused on the hazards that threaten a system than the resilience state of the urban system itself. In urbanism, there is a lack of development of a knowledge base that would permit the understanding and assessment of the resilience of urban landscapes in a systemic and methodical way. Moreover the gap becomes bigger when trying to find theoretical tools to assist urban, architectural and landscape architecture design processes to involve resilience methodologies. On one hand, engineering investigations can be very precise and specific in their tasks but they have a tendency to forget the context of the system analysed, a factor that is extremely important in a resilience analysis. On the other hand, the use of resilience in just a metaphorical way could be a stimulating starting point for a design process but its results could carry a quantity of mismatches that are difficult to avoid. For example, projects and approaches whose aim is basically to show what resilience looks like could tend to forget how resilience works, transforming good intentions into highly incomplete proposals (ARUP, 2012).

Holling proposed that analogies imply risk when transferring knowledge between fields of study, in this case from ecology to urbanism, and that they should only be made when the structure and behaviour of both systems are similar. Particularly, four similarities between urban and ecological systems are 
emphasized: non-linearity, systemic interactions, historical sequence and spatial relationships (Holling \& Goldberg, 1971, p. 226). Consequently the implementation of ecological resilience to urban landscapes should first investigate and show that the built environment of urban landscapes, in theory and in practice, exhibits most of these characteristics and then try to observe if these four similarities behave in the same way in urban landscapes as they do in ecosystems. Only then can a methodical contribution to the application of ecological resilience to urban landscapes emerge or be logically dismissed.

Generating methodical knowledge that could be both an instrument of design and research could soften mismatches in the translation of ecological knowledge to urban, architectural and landscape design investigations and practices. Therefore, the aim of this thesis is to produce a first set of instrumental knowledge that can work as a link between ecological resilience and its utilisation in urban design. Through the implementation of this new knowledge, it should be possible to provide insight into the structures, dynamics and processes that sustain the resilience capacity of the built environment of urban landscapes. Moreover, the same knowledge could be a tool for further research and for assisting the process of design with applications in urbanism, landscape and architecture. However, before achieving these aims it is necessary to start by following the advice of Holling (1971) and first try to see if the translation and implementation of an ecological resilience analysis to urban landscapes is feasible or not.

\subsection{Research question and hypothesis}

The main research question is:

How is it possible to apply the ecological resilience theoretical framework to the analysis of urban landscapes?

The hypothesis is that if the theoretical framework of ecological resilience can be applied to the analysis of the resilience of urban landscapes it can be tested and observed by assessing the dynamics of change in the morphology of built environments. Particularly, the issue is how the dynamics of change occur 
in the structure of a complex adaptive system and how that structure and changes within it are manifested in a landscape and linked with the resilience capacity of the system. Consequently the hypothesis addresses two points.

The first point is that transformations in the morphology of the urban landscape at one scale might or might not produce structural changes at larger scales. In the face of disturbances happening in the built environment, the resilience capacity of the urban landscape will try to maintain the identity of the system by keeping its structure fairly stable at larger scales while allowing variability and change at smaller scales (Holling, 1987). Chapters 4 and 5 deal with these dynamics of change.

The second point is that the heterogeneity of the landscape of a system, its diversity and its resilience capacity are linked. More diverse systems will tend to be more heterogeneous and to show more resilience capacity than less diverse and less heterogeneous systems. Therefore more heterogeneous urban landscapes should tend to experience fewer structural changes due to their higher resilience capacity. Chapter 6 specifically deals with the relationship between heterogeneity and resilience in urban landscapes.

The hypothesis is based on three concepts that structure the theoretical framework of ecological resilience. First, the adaptive dynamics of change within and across scales generate a dynamic hierarchy or Panarchy that can be observed in the way that elements of a landscape are structured, interact and change. Second, the stability state of system can be linked with the persistence or change of its identity. In this case identity means a particular set of relationships and feedbacks between the system analysed and its context. When events occur, the resilience capacity of the system will try to buffer changes until its threshold is surpassed. The shift to a new stability state will imply a new set of relationships and a new identity. Therefore the persistence of the identity of a system would be link with its resilience capacity to absorb changes. Third, the relationship between the heterogeneity of the urban landscape and its resilience capacity can be assessed by using the Textural-Discontinuity Hypothesis (TDH) (Holling, 1992). 


\subsection{Purpose and general objectives of the research}

The main purpose of this thesis is to investigate how it might be possible to apply key concepts from ecological resilience to the analysis of urban landscapes. Methods and results generated by this thesis can contribute to thinking and researching about the resilience capacity of urban landscapes in a comprehensive way. The expectation is that the analysis and diagnosis of the resilience state of the built environment of an urban landscape will contribute to the definition of its resilience capacity and will allow to having an insight into the resilience of a socio-ecological landscape.

The first general objective of this thesis is to investigate whether it is possible or not to find theoretical evidence in other fields of research that sustain the ideas contained in the theoretical framework of ecological resilience and, therefore, validate its application to the analysis of the resilience of urban landscapes. The expected outcome is to find correlations between the key assumptions made in ecological resilience about the way in which ecosystems are structured, change, and work and assumptions made in philosophy, history, economy, urban studies and urban morphology about the way urban landscapes are structured, change and work.

The second general objective of this thesis is to use the knowledge acquired in the theoretical construct in order to implement the criteria for assessment, through discrete methods, in the measurement of the resilience capacity of the built environment of concrete case studies. In order to accomplish this objective three stages are proposed. The first stage in assessing the resilience of urban landscapes is to investigate how elements of urban landscapes are organized and change. The second stage consists of analysing the relationship between identity, persistence and change in relationship with the resilience capacity of urban landscapes. The third stage is to test the spatial implications of the Textural-Discontinuity Hypothesis (Holling, 1992, p. 449) (see section 2.4) through the use of discontinuities in the analysis of the relative resilience of the built environment of urban landscapes.

The third general objective of this research is to add to the understanding of the mechanism that maintains, enhances or lessens the resilience of urban landscapes, particularly by investigating the complex dynamics of change 
through observations and analysis of transformations in the urban form. In this research it is desired to present a possible interpretation of ecological resilience and a path to its application in the reality of urban landscapes. The hope is the theoretical framework presented here will be a reference that can be used or further developed by managers, urban planners, landscape and architectural designers into a methodology or theoretical design tool, or a new field of research.

All of these objectives represent contributions to filling the gap in knowledge created by suggesting that resilience theory can be applied to socioeconomic landscapes. The additional hope is that the knowledge created can become useful for the practice of architecture, urbanism and landscape design.

\subsection{Methodological approach}

The interdisciplinary nature of this research links different fields of study in order to extrapolate concepts from ecology to urban studies. Therefore, the first challenge was to design a methodology with which to extrapolate concepts from one discipline to another. This methodology is necessary to make possible a comparison between the two systems and also to abstract and isolate the resilience dynamics of change. The foundation for extrapolating concepts from one field to another by using different fields of study is based on the theory of incompleteness as posited by Gödel (Chen, 2004). This philosopher argued that there is no single system of logic that is able to prove its own consistency (Catedra Manteola, 2006) therefore every translation will always be incomplete. A possible way of reducing the margin of incompleteness could be through increasing complexity and redundancy by having multiple viewpoints. Consequently, in order to transfer information from one discipline to another, it is necessary to have a complex approach in which a second body of knowledge acts as an interface, in this case an interface between ecology and urbanism. The challenge is to find the course in this approach that will minimize mismatches.

As Edgar Morin (2001) has proposed, in order to tackle complexity, in this case by reducing the margin of incompleteness in the extrapolation of concepts from ecology to urban studies, the design of a complex approach is needed. The complex approach proposed here is a way of assessing the complexity of 
complex adaptive systems in a non-linear way. Complexity here means understanding the simple and the complex, qualitative and quantitative, linear and non-linear, big steps and small steps, all at the same time.

The complexity of the methodology must be suitable so as to avoid simplifications that will reduce the richness and potential of the resilience thinking. For this reason, instead of making the analysis of only one case study with one method, using a single specific variable, in this thesis resilience thinking was used to inspire the design of a methodology that could tackle the complexity and adaptability of the systems analysed. The complexity of the methodology can be observed in the consideration of multiple disciplines, case studies and methods. A complex methodology is based on the interrelationship between multiple theories assembled in a single theoretical core that is tested in discrete case studies, using different methods based on alternative assumptions that are linked with the concepts and hypothesis stated. This approach can generate diverse results with the potential to enrich the theory at different levels and from more than one viewpoint. A complex methodology implies conceiving of the design process of the methodology of the thesis as a research project. From this point of view, the design of a methodological approach could be understood as an anticipatory design (Healey, 2007). This epistemological knowledge could then become the foundation for producing a theoretical framework for analysing and diagnosing the resilience of SELs in design.

The methodology is based on a set of theoretical cores (cores 1, 2 and 3 in fig. 1-2) that are linked to the main theoretical background of the thesis (context, ontology and philosophical approach in fig. 1-2). The theoretical background provides the foundation and criteria for assessment that will be used throughout the thesis to tackle different subjects concerning the application of ecological resilience in the analysis of the resilience capacity of urban landscapes. It is the conceptualization of a context in which the study of the resilience capacity of urban landscapes is the main subject. At the same time it is the bridge that links concepts with their application in a context. The theoretical background uses contributions from economics, ecology, philosophy, history, urbanism, architecture and landscape design studies in order to validate and support the application of ecological resilience to urban landscapes. The theoretical 
background is constituted by the context, ontology and philosophical approach of the thesis. It is a production of knowledge that implies a way of seeing the world, an understanding of reality (Sarquis, 2003a) and a philosophical approach that becomes an interface, and an instrumental theory (Sarquis, $2003 \mathrm{~b}$ ). The pursuit of the theoretical background is made in order to produce a way of thinking about resilience in urban landscapes and also about the criteria for assessment.

In order to test the interpretation of the key points of the theoretical framework of ecological resilience, and the internal validity of the hypothesis, two case studies are used (section 1.10). The aim is to test and to develop different methods in order to find evidence in support of the assumption it is possible to assess the resilience capacity of urban landscapes. For this reason, methods and results are produced in order to analyse a particular subject (related with a specific set of concepts derived from the theoretical background of the thesis) in each case study (cores 1, 2, 3 in fig. 1-2). These, in turn, become evidence to support and exemplify how to apply the ecological theory in urban landscapes.

The complex approach designed emphasizes the generation of multiple methods, and multiple theoretical cores that are created in order to research each subject within its case study. Each theoretical core deals with one key aspect of the resilience theory, an urban landscape, a method conceived to analyse a selected set of concepts, and a group of results obtained from the analysis. Therefore, each case study interacts with the theoretical background of the thesis by providing feedback through the methods, and results produced in each research sub-study. The set of feedbacks provided in the conclusion of each case study will contribute to enhancing the theory and practice of the resilience capacity of urban landscapes by feeding back into the theoretical background of the thesis. Consequently, every analysis, and particularly the methods and results obtained, become evidence of the feasibility, limitations and potential of the ideas and criteria for assessment proposed. 


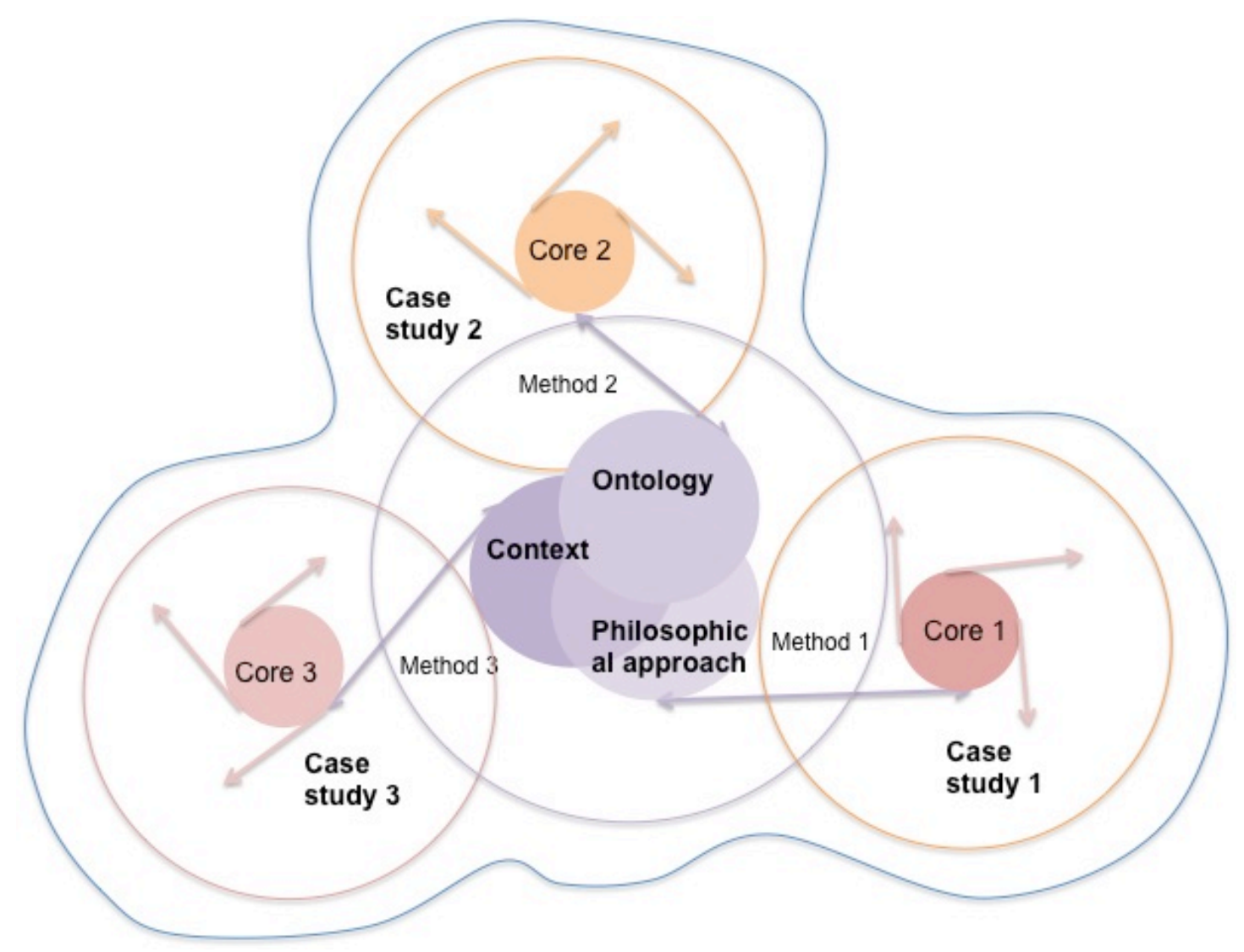

Figure 1-2. A complex approach to the research

\subsection{Case studies}

In the context of the complex and non-linear methodological approach implemented, case studies are used as the means to create the encounter between the theoretical background of the thesis and its possible implementation in concrete scenarios. The objective is to use case studies as part of an iterative process where key parts of the theoretical background are explored, thereby producing different methods and results in each case. As a result of this iterative process, every case study helps to explore the research question through different points of view.

If the proposed way of thinking about urban resilience is to be applied in case studies, it should be tested and compared in different cities, with discrete histories and cultural backgrounds. Thus, two case studies were selected: the east side of Auckland CBD in New Zealand and the neighbourhood of Nezu in Tokyo, Japan. It is important for the theoretical background to analyse, characterize, and compare the dynamics of change, and identity of a young city 
like Auckland with another urban landscapes that has passed through a longer process of development, such as Nezu in Tokyo.

The first area of study, the urban landscape of the east side of Auckland CBD, was chosen as a case study because Auckland is the biggest and most populated city in New Zealand and therefore it is an urban referent for the country. At the same time there is sufficient accessibility to archives and information. The methods used in the analysis of the morphology of Auckland demand much documentation, particularly maps with building footprints, plots, and streets in different periods. Due to its importance, the history of the urban landscape of Auckland is better documented than other cities in New Zealand, such as Wellington. Another reason for choosing Auckland as a case study is the fact that it is not an old city. This characteristic makes its urban landscape a good sample of a contemporary built environment in which the transformative process has taken effect in a shorter period of time in comparison with other European, Latin American or Asian cities.

The east side of the CBD of Auckland was selected as an area of study because its urban landscape is linked with the origin of the city, consequently it can be followed and assessed at different periods. Moreover, the east side of the CBD contains in a small area a good and diverse sample of spaces that reflect different periods of the history of the city.

The second area of study, the urban landscape of Nezu, a neighbourhood of Tokyo in Japan, was chosen in order to contrast directly with the characteristics of the east part of the CBD of Auckland. While the urban landscape of Auckland is young, modern and linked with an Anglo-Saxon culture, the urban landscape of Nezu is old with a strong identity that forms the focus of attraction of the place. The possibility of access to maps and information about the evolution of the urban landscape of Nezu created a perfect opportunity for analysing a case study that is the opposite to the area selected in Auckland. At the same time the urban landscape of Nezu is an appropriate sample for analysing resilience capacity in terms of the identity of a place. Nezu has suffered many fires and times of destruction in its built environment but stills holds its shitamachi identity. Therefore its urban landscape seems to show a high resilience capacity to buffer change and still persist. Another interesting 
characteristic of the urban landscape of Nezu is that it does not contain public open green spaces or sidewalks, both of which occupy a significant area in the urban landscape of Auckland.

The contrasting reality and characteristics of the urban landscape of the east side of Auckland CBD and Nezu offer a good opportunity to observe different points of the theoretical background of the thesis and also to make a comparative analysis of the resilience capacity of both urban landscapes.

\subsection{Structure of the thesis}

The content of thesis is divided into three parts and seven chapters that correspond to the three general objectives of the thesis, assessing the theory, practice and contributing to the understanding of the resilience of urban landscapes (Fig 1-3).

The first part of thesis is its theoretical background that is discussed through chapters one, two and three. The three chapters set the baseline and try to expose the foundation of how to think about the assessment of the ecological resilience of urban landscapes. The theoretical background of the thesis looks for evidence in other fields of study, apart from ecological resilience and urbanism, to validate the idea of extending the theoretical framework of ecological resilience to urban studies. The aim of the three chapters is the generation of the criteria for assessment that will be used to assess the application of ecological resilience to urban landscapes. The final aim is to show that, in theory, the hypothesis is valid and achievable. This theoretical core has three main components: the context and purpose of the thesis (chapter 1), the ontology (chapter 2) and the philosophical approach of the research (chapter 3).

Chapter one sets the context, importance and scope of the study. It describes the development of the argument and description of the manner and progress of the thesis. The aim of chapter one is the produce the research question and hypothesis that will guide the research.

Chapter two deals with the fundamental theory that tries to build the ontology of the thesis. It establishes a set a common ground between different subjects of study and disciplines. The ontology of the thesis was produced by assembling subjects of study related to evolutionary processes in social and 
natural systems that have spatial implications in cities, urban landscapes and particularly the built environment. Using this approach, a strong intersection was established between ecological resilience, assemblage theory (De Landa, 2006) and morphogenetics (Whitehand, 1981).

Chapter three describes the philosophical approach of the thesis. It connects a set of concepts, derived from the subjects of study assembled in the ontology, and the way in which they can be linked to analyse the resilience of the built environment of urban landscapes. The aim of the chapter is to link key concepts that set a common ground between ecological resilience, assemblage theory and morphogenetics, in order to define the criteria for assessment.

The second part of the thesis, chapters four, five and six, corresponds to the practical dimensions of the application of ecological resilience to the analysis of urban landscapes. This second part describes, through the development of different methods and case studies, how the ontology and philosophical approach of the thesis can be applied to urban landscapes. Therefore the aim of this part is to show evidence of how to apply key concepts of the ecological resilience theoretical framework to the study of urban landscapes. Each chapter (chapters 4, 5, and 6) investigates and discusses the assessment of ecological resilience in urban landscapes in accordance with different key topics discussed in the ontology and using the set of criteria developed in the philosophical approach.

In chapter four, the subject is the understanding of the resilience capacity of urban landscapes by assessing the dynamics of change in key elements of the built environment. The method used is based on the production of timelines. The aim is to illustrate the heterogeneity of the built environment, its multiplicity; and the different dynamics of change happening at different scales. This set of topics is related with the understanding of Panarchy in urban landscapes. The case study used is the east part of the urban landscape of Auckland CBD.

In chapter five, the subject is the relationship between the resilience of the built environment and the identity of the place. The concept of identity is used as a metaphor to characterize the ecological concept of a stability state in the built environment of an urban landscape. The aim of the chapter is to find 
evidence that the resilience capacity of an urban landscape can be observed and assessed by measuring changes in particular elements of the built environment (building footprint BF, plot PL, and block BL) against changes in the whole structure of the system (shifts in the stability state/identity of an urban landscape). The method developed is a comparative analysis using percentage of change difference before and after events. The case study analysed is Nezu, a neighbourhood in Tokyo, Japan. This part of the study was done in collaboration with Milica Muminovic from Keio University of Tokyo, Japan.

In chapter six, the subject of study is the relationship between the heterogeneity of the urban landscape and its resilience capacity. The topic is related with the observation and assessment of the Textural Discontinuities Hypothesis, a key point in the theoretical background of the thesis. The aim is to measure the relative resilience by quantifying and comparing changes in the structure and heterogeneity of two built environments. The method produced is based on the use of discontinuities to assess relative resilience. The chapter compares the dynamics of change in one sector of Nezu (Japan) with another sector in the east side of Auckland CBD. The chapter synthesizes in one method a way to measure and to compare resilience dynamics.

Finally, chapter seven reflects and discusses findings and conclusions from each part of the thesis. The aim of this chapter is to collect and to summarize the results produced by discrete methods in different subjects and to interpret them using the theoretical background of the thesis. At the same time it describes the advances produced in the theory and practice of the analysis of the resilience capacity of urban landscapes. The chapter ends with descriptions of the weaknesses inherent in the methods implemented and highlights the potential of the research to be developed in future projects. 


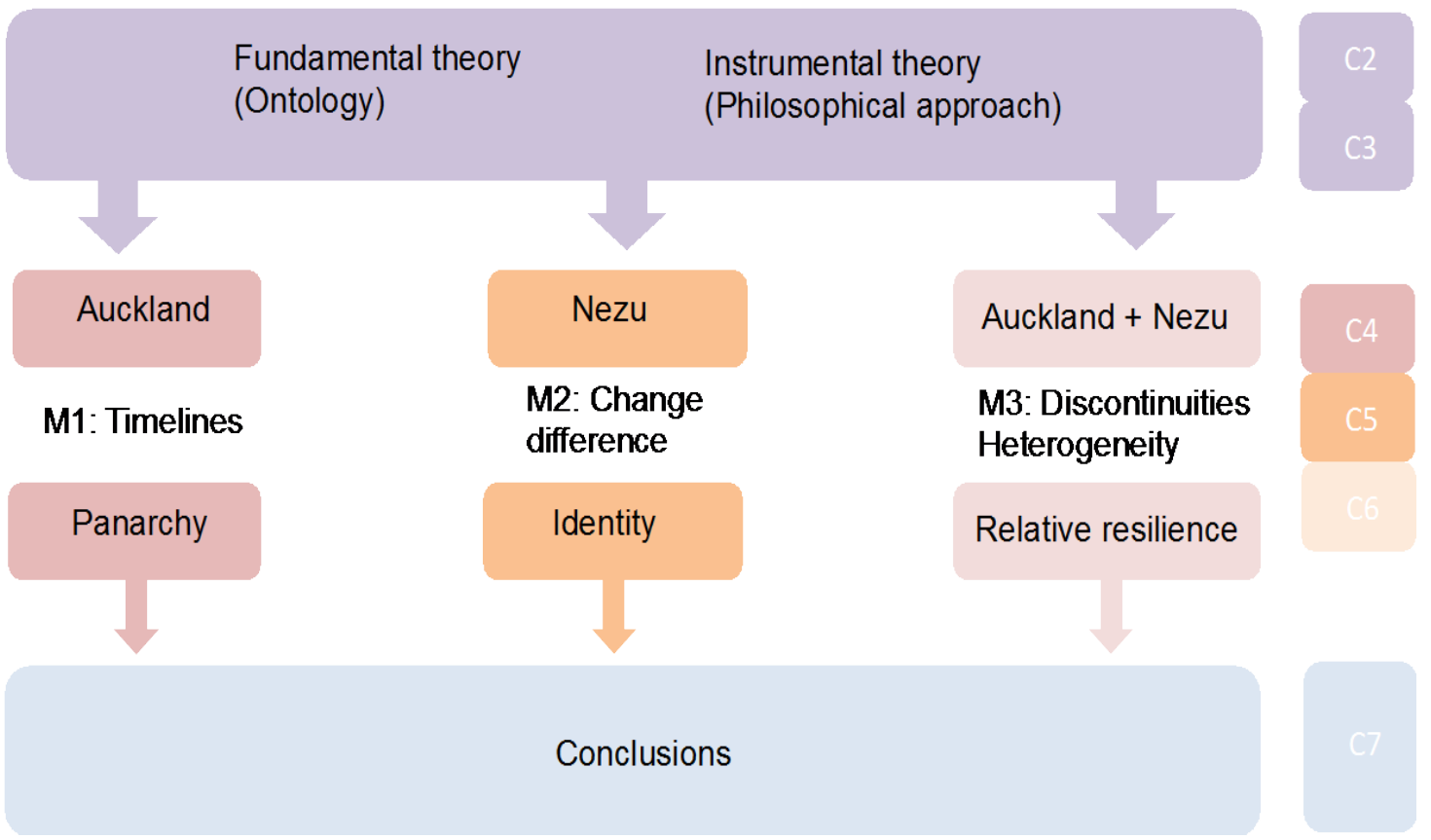

Figure 1-3. Structure of the thesis

\subsection{Data collection methods}

The methods used in this thesis were based on the analysis of the morphogenesis of urban landscapes. The necessary information to perform these morphological observations is linked with the data collected from historical maps of the urban landscape that are then analyzed. The first step is linked with the research, identification and selection of the appropriate maps. The criterion for selection is to look for historical maps that contain the following categories of information: streets, plots, blocks and building footprints (in the same map). When a map lacks one category of information (usually building footprints) it was considered as a reference but not all such maps are used in the analysis. Aerial pictures were not collected due to the lack of precision they offer when redrawing them as maps in two dimensions. All the maps have been previously scanned in colour and with high resolution (300 dpi). Only digitized copies were used in the data collection. 
The second step in the data collection consists on redrawing the maps. Maps were redrawn, using software packages focused on the management of geographic information, especially QGIS (in the case study of Auckland) and ARCGIS (in the case study of Nezu). Firstly scanned maps were geo-referenced to the contemporary map of each area. In the case study of Nezu, data from the Center for Spatial Information Science (The University of Tokyo) was used. Secondly, maps were redrawn using vectorization tools contained in QGIS and ARCGIS. Buildings footprints, plots, blocks and streets were polygonized in different layers. Each layer has an attribute table that provides information about the number, name and area $\left(\mathrm{m}^{2}\right)$ of each feature (i.e. every block, plot, block or street). Snapping options were used to give more accuracy to the drawing of polygons when they were close to each other. Buildings footprints were draw one by one trying to be as accurate as possible. Blocks were drawn including sidewalks, where they exist. Streets were also redrawn as lines taking the centre of the road as a reference. Thirdly, all the information was exported to Microsoft Excel or to Weka software (in the case of cluster analysis) to make the necessary calculations. Every layer (plots, blocks, buildings footprints and streets) was exported one by one and year per year to the software used for making the relevant calculations. When it was necessary, the importation that was processed in other software was imported to QGIS and ARCGIS in order to be joined as a new column in the attribute table of each layer. In this way final maps were produced depending on the method, aim and analysis performed. 


\section{Ontology: Ecological resilience, philosophy and urban morphology}

\subsection{Introduction}

This chapter aims to be an active integration of concepts from different fields instead of a literature review of ecological resilience. Connections in this way will produce new opportunities for analysis in further research. The epistemological approach proposed in this ontology maps the overlaps between assemblage theory, complex adaptive systems, morphology and other urban studies using as a framework the set of key concepts derived from ecological resilience studies. The result is an episteme that reveals a set of connections and bridges between theory of place, assemblage, urban morphology and resilience. It finds evidence that resilience thinking can be used to observe the evolution of the built environment in order to measure the dynamics of change in an urban landscape. At the same time, it opens new areas of research that result from the intersection of the assembled fields.

The holistic direction taken here explains key concepts of ecological resilience with parallel concepts in other fields. The key concepts from the ecological resilience theoretical framework discussed in this chapter explain how complex adaptive systems change and organize themselves in a Panarchy. This chapter proposes an epistemological approach that shows that the concepts and hypothesis generated in ecological resilience around the dynamics of change of complex adaptive systems could be suitable for use in the analysis of urban landscapes because both systems are complex and exhibit adaptive behaviour in the face of change (see section 2.2.1).

\subsection{Ecological background}

Resilience is a recognised property of eco-systems (Folke et al., 2004; Gunderson, 2000) and it has been proposed, but not yet fully proven, that human urban systems behave in a similar way (Alberti, 2009; Garmestani, C. Allen, \& Gallagher, 2008). Here, resilience is defined as the capacity of a system to absorb disturbance and reorganize while undergoing change, so as to retain essentially 
the same function, structure, identity, and feedbacks (B. Walker \& Meyers, 2004). This way of thinking about ecological systems (B. Walker \& Salt, 2006) can make a solid contribution to the analysis and understanding of how cities can be prepared for and mitigate unpredictable changes while continuing to evolve in such a way that their development does not compromise their resilience.

The objects of study of ecological resilience are socio-ecological systems (SES), which are systems that are constituted by the interaction of human beings and nature (B. Walker \& Salt, 2006), like ecosystems and cities. Resilience is trying to understand changes in socio-ecological systems from the perspective of a nature evolving (Gunderson \& Holling, 2002). Moreover it can be said that ecological resilience is trying to generate a hypothesis about the way in which ecosystems are structured and how they work.

The theoretical framework of ecological resilience explains change and structures in ecosystems through a set of key concepts: multiple equilibrium states, adaptive and cyclical change (adaptive cycle), nested set of adaptive cycles (Panarchy) and the production of a complex landscape when heterogeneity is exposed in the discontinuous texture of the landscape. These concepts will be developed in section 2.2 and then compared with similar concepts in philosophy (see section 2.3) and urban morphology (see section 2.4).The behaviour of complex adaptive systems

\subsubsection{The behaviour of complex adaptive systems}

In order to investigate the possibility for using the assumptions and discoveries made in ecology and other fields of study about the resilience of the built environment of urban landscapes, it is necessary to observe first if the behaviour of the built environment of urban landscapes is similar to a complex adaptive system (CAS) (A. Holland, 1980; J. Holland, 1992).

The study of CAS in ecology and urban landscapes is relevant to understanding how structures work, particularly structures that have emerged from the interaction of patterns. Levin (1998) and Holling (1971) highlighted some of the characteristics of a CAS in ecology. These are aggregation, or how elements are grouped into bigger structures (individuals into a population), nonlinearity (rules of interaction change as the system evolves producing 
unexpected results), diversity (more diverse systems are more robust and better prepared to buffer disturbances) and flow (interchanges between elements in a system). All these characteristics of a CAS are implicit in the processes of change described by the theoretical framework of ecological resilience.

This understanding is essential when making measurement of the dynamics of change of urban landscapes because it permits having criteria to define whether a system is behaving in a complex adaptive way or not. If these complex adaptive behaviours are found in the processes of change related with the built environment of urban landscapes it could be evidence for applying the theoretical framework of ecological resilience to urban landscapes.

\subsubsection{Stability states, multiplicities}

Holling defined the behaviour of ecosystems as the result of two properties: resilience and stability.

"Resilience determines the persistence of relationships within a system and is a measure of the ability of these systems to absorb changes of state variables, driving variables, and parameters, and still persist. (...) Stability, on the other hand, is the ability of a system to return to an equilibrium state after a temporary disturbance. The more rapidly it returns, and with the least fluctuation, the more stable it is." (Holling, 1973, p. 19)

The implications of this definition are related with the idea that ecosystems can work in more than one stability state (Gunderson, C. Allen, \& Holling, 2009; Holling, 1973). The persistence in time and space of a set of relationships between a system and its environment defines a type of equilibrium state. The equilibrium point of a system can be illustrated as the result of a continuous resolution between internal and external opposite forces that define a shifting balance (Folke et al., 2004). 

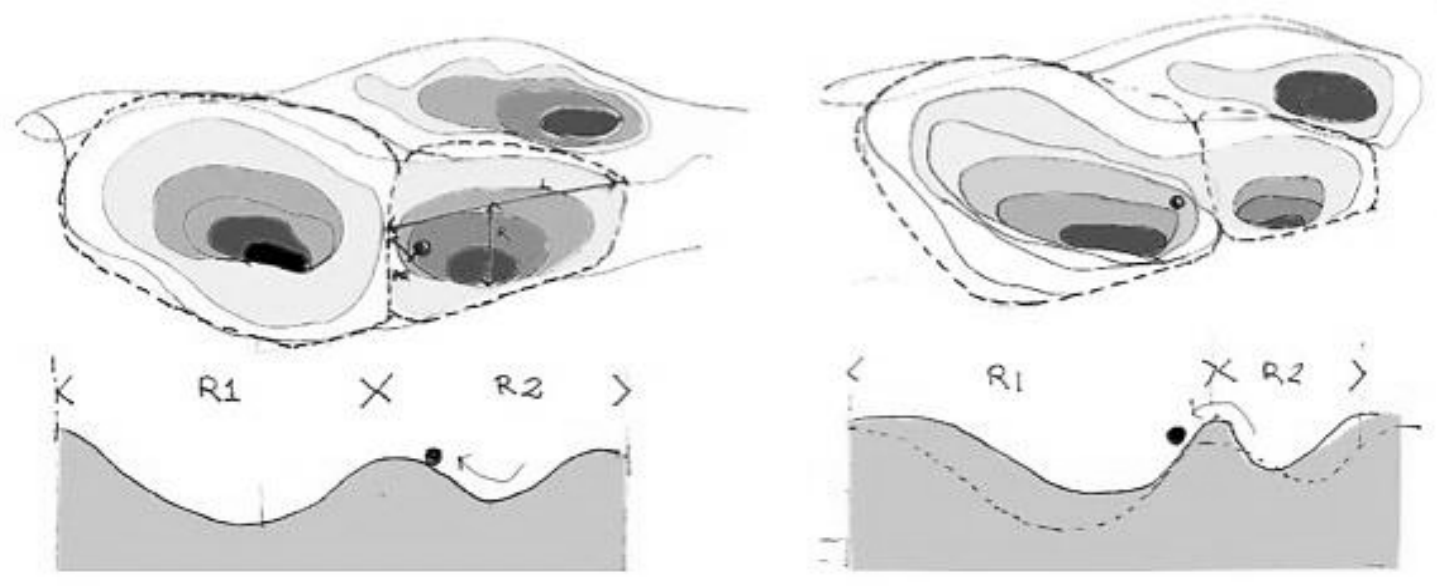

Figure 2-1. The multiple stability states of a landscape based on (Folke et al., 2004)

According to ecological resilience, every system is working in an environment around an equilibrium point situation that is defined as a domain of attraction. In the context of the system there exist multiple equilibrium points, each one with its own domain of attraction (Fig. 2-1) (Peterson, C. Allen, \& Holling, 1998). A system can be working within a familiar environment and suddenly shift to a different one if its resilience capacity is exceeded. Therefore, the resilience capacity is what keeps a system within the thresholds of an equilibrium point when disturbances try to make it shift. A shift to a new stability state can be something desirable or not (B. Walker \& Meyers, 2004). 


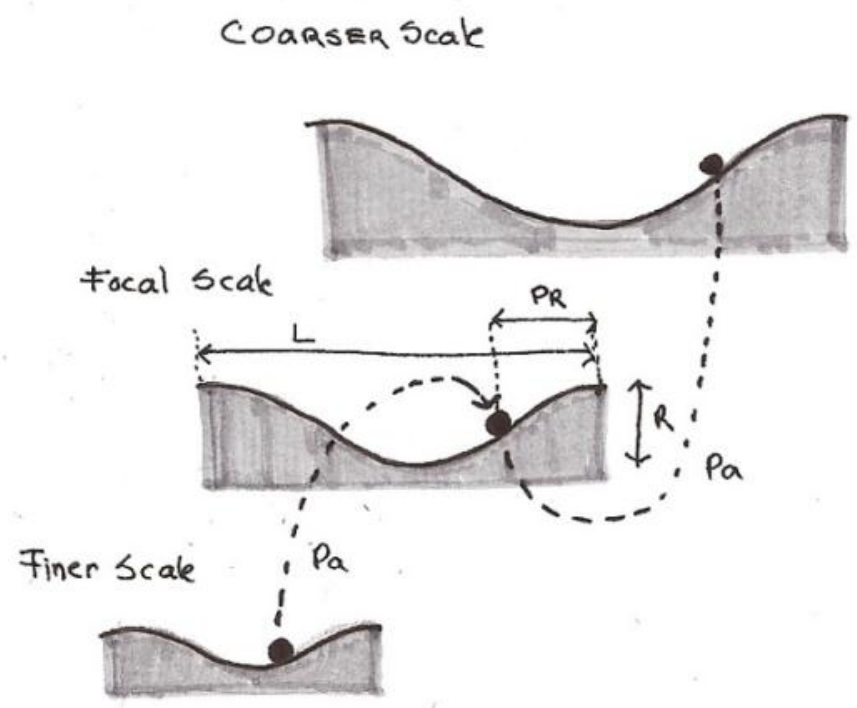

Figure 2-2. Multiple stability states and cross scales dynamics based on (Folke et al., 2004)

Resilience and stability are opposite and complementary properties that characterize the adaptation process of complex systems (Gunderson, 2000). While stability is trying to fix and control the feedbacks of a system, it is also making their links more rigid and by the same reason less resilient to unpredictable changes (B. Walker \& Salt, 2006). A complex system is hardly in a single equilibrium point for long. So if the stability is interrupted two things could happen. First, the resilience of the system allows it to keep on working in the same stability state by making some adjustments in its performance; second, the resilience of the system is exceeded and the system shifts to a different stability state (Gunderson \& Holling, 2002). This is termed a regime shift (Folke et al., 2004) (Fig. 2-2). In this situation, the predictability is very low, the opportunities unknown and as a result, the system becomes unstable. The resilience attributes of a system have to be evaluated in a wider perspective, always considering the importance of the internal dynamics of a system in relationship to external influences from the environment of the system (Resilience Alliance, 2007b).

\subsubsection{Adaptive cycle}

Ecological resilience explains that change in ecosystems happens in cycles that are linked with non-linear processes of development and decay (Gunderson 
\& Holling, 2002). The metaphor of the adaptive cycle is used by the resilience theoretical framework to illustrate in four phases the implications of change at discrete spatio-temporal scales (Holling, 1992). In the adaptive cycle (Fig. 2-3) the first phase (exploitation) is a period of rapid development of accumulated resources. A system is not very stable but highly resilient. The second phase (conservation) is characterized by an accumulation of potential and complexity that a system gains due to its progressive connectivity and functional specialization. Consequently the system acquires more stability but also more rigidity; this is a situation that lowers its resilience capacity to the point that small surprising events can cause huge problems. As a result, the system becomes fragile and any unpredictable change can make it collapse. The third phase (release) is characterized by an important loss of connectivity and potential in a short period of time. After the system collapses it could either restart a new cycle through a reorganization process (reorganization) or shift to a different stability state (Gunderson \& Holling, 2002, pp. 40-47).

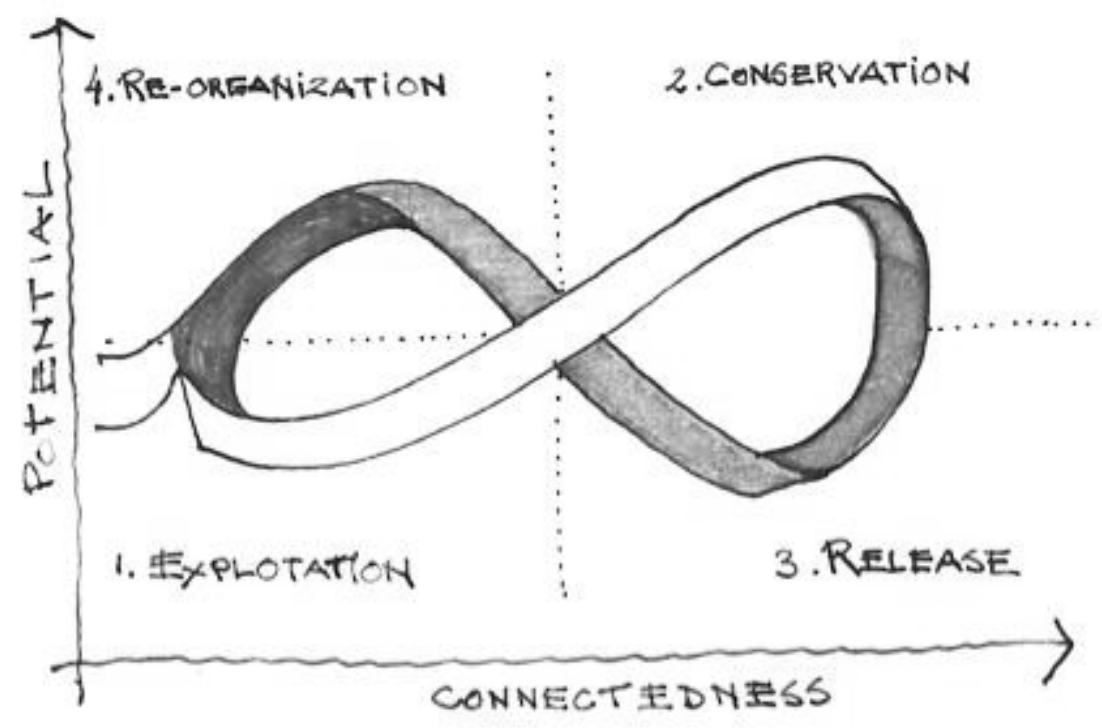

Figure 2-3. The adaptive cycle based on (L. H. Gunderson \& Holling, 2002)

The adaptive cycle can be synthesized into two trends: the "front loop and back loop" (Gunderson \& Holling, 2002, p. 47). The front loop, (white band in Fig. 2-3), consists of the phases from exploitation to conservation, and could be exemplified by the accumulation of capital, slow incremental growth, 
predictability and stability needed for the identity of a place to be consolidated. The back loop, (dark grey band in Fig. 2-3), consists of the phases from release to reorganization (Gunderson \& Holling, 2002), and is the dynamic that produces opportunities from a crisis (Schumpeter, 1994). In urban landscapes it can be related with what enables the identity of place to evolve. In the same way as for the processes of territorialization and deterritorialization in assemblage (see section 2.3.1), in the adaptive cycle, both loops cannot be maximized at the same time, they occur in sequence. While complexity and stability increases, the same processes make its identity more rigid, unstable and unpredictable, reducing its resilience capacity (Holling, Walker, Carpenter, \& Kinzig, 2004). This is the resilience problematic, which lies between the front and back loops, between persistence and renewal.

\subsubsection{Panarchy: change within and across scales}

In ecological resilience every adaptive cycle is aligned to one spatiotemporal scale. In ecology, the concept of scale is different from that of geography or architecture because it describes "the spatial extent of a structure or process of interest and its temporal frequency" (C. Allen \& Holling, 2010, p. 1), implying that a scale defines not only the extent of an entity in space but also the frequency of processes related with that entity. For example, a leaf occupies a small space, has a short life cycle but appears frequently in a landscape; however storms happen less frequently but affect more space. Complex systems are constituted by more than one scale, each one belonging to a distinct adaptive cycle. A nested set of adaptive cycles constitutes a Panarchy (Gunderson, Garmestani, \& C. Allen, 2009, p. 1). In ecological resilience, Panarchy helps to conceptualize the structure of ecosystems as a dynamic hierarchy where adaptation occurs not only within one scale of an adaptive cycle but also across scales (Fig 2-4). At large scales, changes are slow and not so frequent because their processes are mainly managed by slow variables; while at small scales, changes occur more often and they happen faster due to the predominance of fast variables (Gunderson \& Holling, 2002, pp. 69-72). 


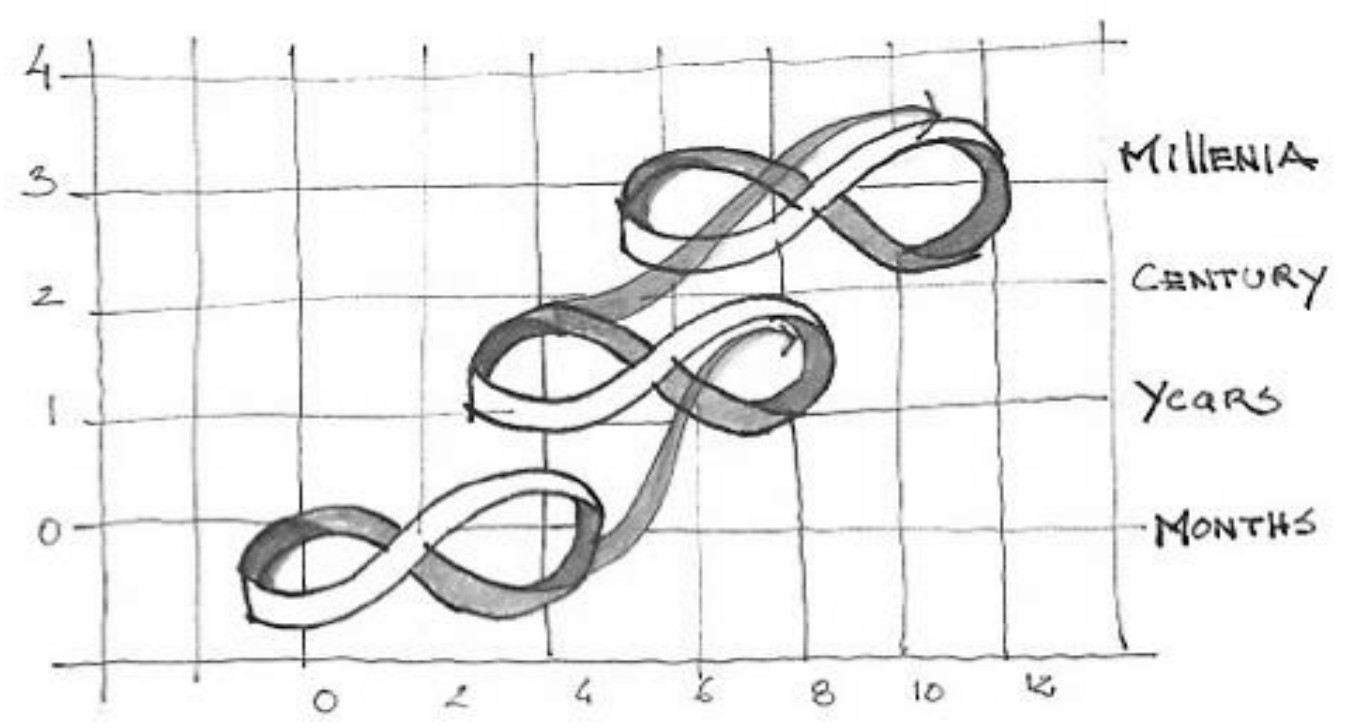

Figure 2-4. A Panarchy based on (L. H. Gunderson \& Holling, 2002)

All these interactions produce emergent changes, which help the system to self-organize and to learn. In a Panarchy changes across scales happen due to two processes known as "revolve" and "remember" (Gunderson \& Holling, 2002, pp. 75-76). Revolve is a bottom-up process that starts at lower scales in the Panarchy with the possibility of cascading up and having an impact at bigger scales, a situation that does not occur frequently. In contrast, remember is the opposite force, a top-down process that tries to keep the system stable and is driven by slow variables. Systems that do not allow revolves can stagnate and become rigid because of a progressive loss of resilience. Systems where revolves are frequent are not stable enough to sustain a development. Consequently, a Panarchy demands a broad understanding of the dynamics of change within and across scales, which means that the general resilience of a system is as important as the comprehension of the specific resilience of the system at focal scales (Gunderson \& Holling, 2002).

\subsubsection{The Textural-Discontinuity Hypothesis in ecological resilience}

The evolutionary processes in a socio-ecological system are adaptive, thus implying that they are not linear and continuous but that they are non-linear and discontinuous in time and space; consequently they produce a multi scale dynamic hierarchy that is named a Panarchy (Levin, 2005). The theoretical 
similarities found between dynamics of change in ecosystems and urban systems permits the inference that all discontinuous processes generated by adaptive cycles and cross-scale interactions in a Panarchy will not only reflect the discontinuous processes and structure of a socio-ecological system (Gunderson \& Holling, 2002) but will also produce a discontinuous socio-ecological landscape. All assumptions made about the possibility of having multiple stability states in a system, adaptive cycles, and the production of a dynamic hierarchy like Panarchy, need to be proved and justified in order to constitute a theory.

In ecology attempts to prove the existence of SESs organized by a Panarchy are being made through the "Textural-Discontinuity Hypothesis" (TDH) (Holling, 1992, p. 1). This hypothesis states that discontinuities in the size of animals should reflect the discontinuous processes that structure the landscape. If a landscape and the organisms living in that landscape are organized at different scales and they have interactions between scales, the result of all these processes will be a "lumpy landscape" (Holling, 1992, p. 78; Krugman, 1996, pp. 44-45). In this way a relationship between the heterogeneity of a landscape and the processes that produce that heterogeneity are established (Gunderson \& Holling, 2002). The ecological resilience approach states that the heterogeneity of a landscape is not a casual event, it is the product of multiple and heterogeneous processes that occur as described in the adaptive cycle and Panarchy. Moreover, scientists in this field (Gunderson \& Holling, 2002; Krugman, 1996; Levin, 2005) propose that textures produced in a heterogeneous landscape reflect internal processes, and for this reason they reveal information about the performance, organization and structure of the system. It is thus possible to infer that the analysis of the heterogeneity of a socio-ecological landscape should give information about the state of the socio-ecological system.

According to the TDH, animal body sizes are related to behavioural choices and availability of landscape resources at specific search scales. Species of similar sizes might exploit resources at similar scales in a landscape. If landscapes are heterogeneous or lumpy, their discontinuous resource distribution will tend to cluster animals of similar sizes in lumps, at different scales of space and time. Bigger organisms will tend to occupy bigger spaces but 
will also be less numerous than smaller organisms that will forage in smaller spaces but to be more numerous in the landscape. At the same time processes of change in elements of the landscape at small scales tend to occur faster than processes of change transforming bigger elements at bigger scales of the landscape (Fig. 2-5). The heterogeneity of the landscape will, in this case, be a cause for clustering more organisms in richer zones, where the stability is high and resources abundant, than in transition areas where resources are scarce and variability high. The product of that uneven occupancy and exploitation of resources in a landscape will make the heterogeneity of the landscape even more discontinuous. The TDH proposes that there should be a geometrical correlation between sizes of the organism that exploit one parcel of a landscape and the texture of that landscape (Holling, 1992).

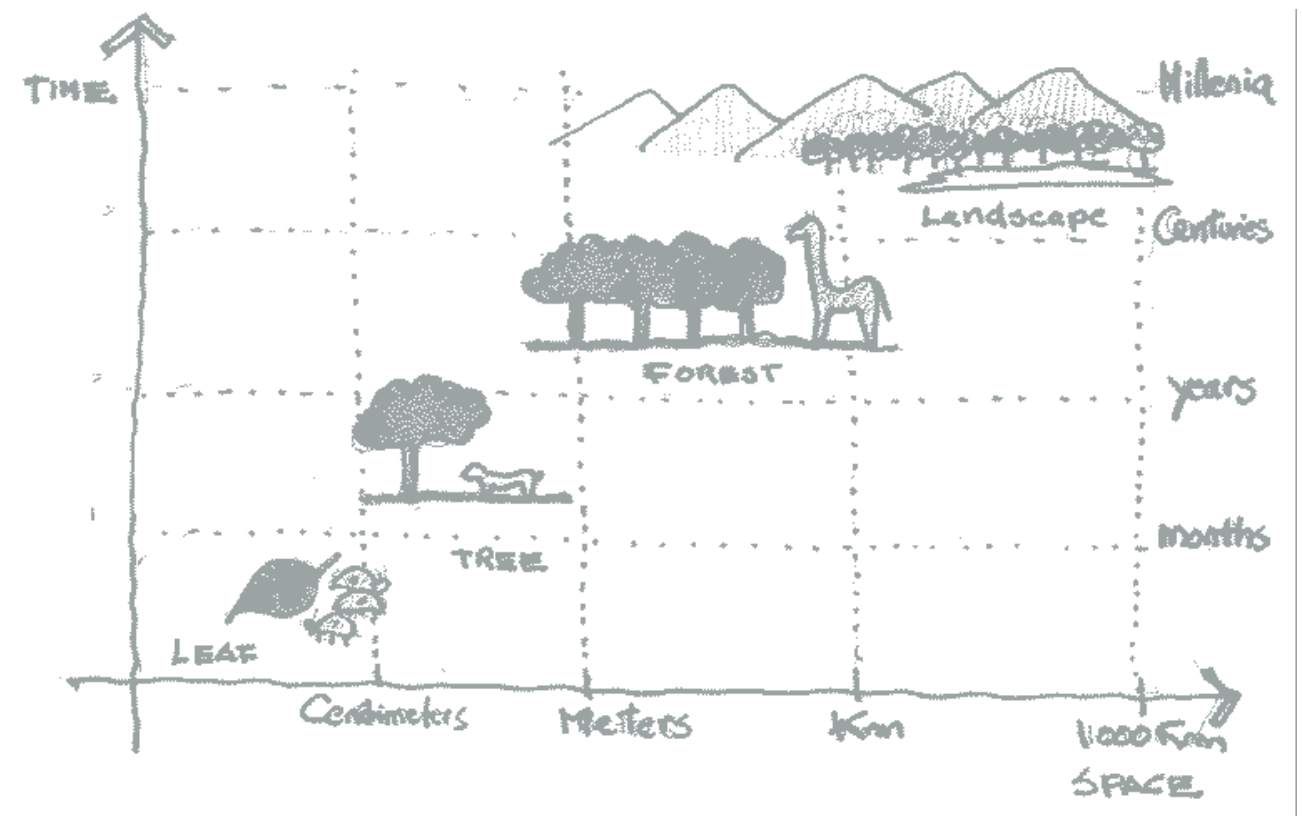

Figure 2-5. The Textural-Discontinuity Hypothesis in ecosystems

As a result of these processes of entrainment (Fig. 2-5), animals tend to be organized in aggregations of species with a similar body-mass that forage at the same scale (Holling, 1992). When anthropogenic perturbations, for example, affect key processes they could have an impact on the Panarchy and consequently on the distribution of resources in the landscape (C. Allen, Forys, \& Holling, 1999). Changes in the availability of resources will produce changes in 
the composition of the clusters. For this reason, the stability of a landscape should affect the morphology and functional diversity of its constituent elements. The pattern of aggregations in the landscape should change affecting the size of the animals that inhabit the landscape. Therefore by measuring transformations in the body-mass size and distribution of animal communities it should be possible to track structural changes that are related with the present and future resilience of the system (Holling, 1992). The analysis of size changes and functional diversity, at each scale of the landscape, should reflect the dynamics of adaptation of the communities that inhabit a landscape. An understanding of how a lumpy landscape behaves at different scales of space and time, allows these to become key variables to test the resilience capacity of a landscape in the presence of unpredictable changes (P. Allen, 1997). In this way, the TDH could help in improving the urban understanding of how the variability of resources in a landscape produces similar discontinuities in the morphology and richness of its elements.

\subsection{Philosophical approach}

This approach aims to find analogies between assemblage theory and the

resilience of complex adaptive systems through analysing the evolution of the built environment (Fig. 2-6). Assemblage theory and the concept of ecological resilience are merged together creating common ground in order to enhance the discourse on identity in the stability of urban landscapes. The objective is to find parallels between socio-ecological systems and assemblages in socio-ecological landscapes in order to describe the built environment of urban landscapes as a complex adaptive system with a resilience capacity. 


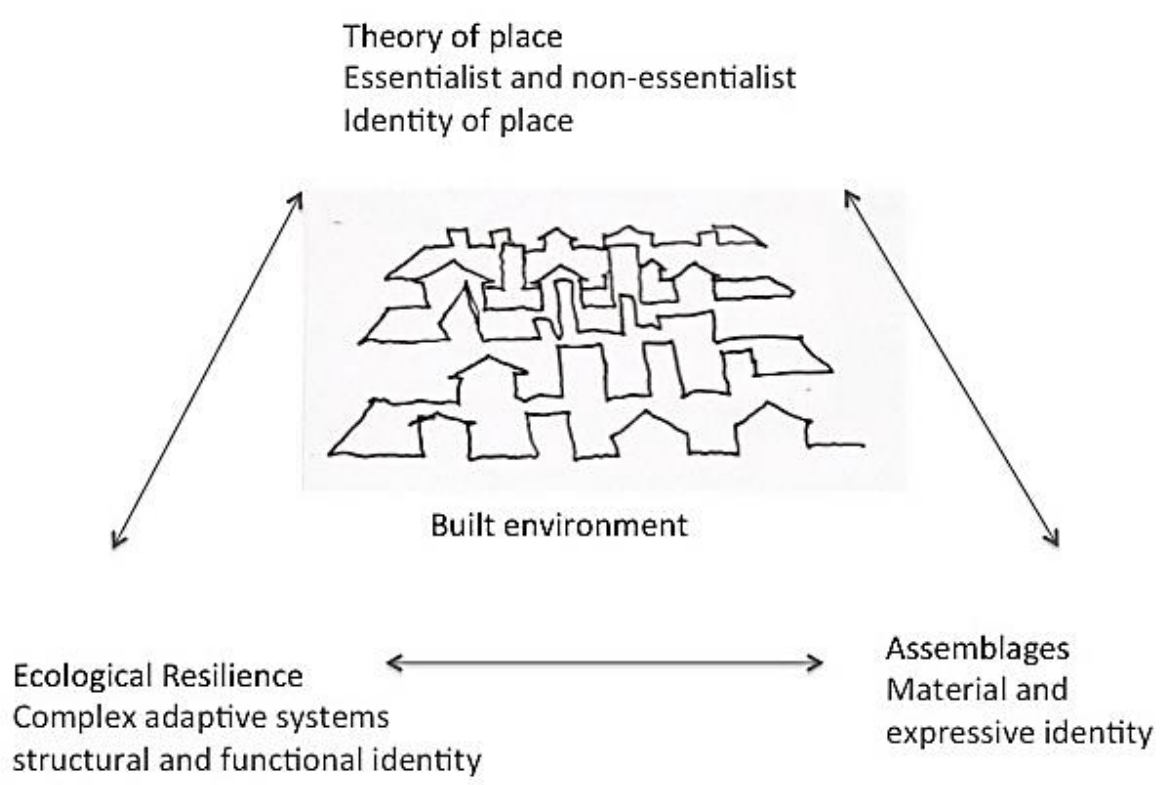

Figure 2-6. Dimensions implied in the proposal for an assembled identity

In urban and architectural studies, particularly in place theory, the idea of the persistence of an essence has traditionally been one principle used to define identity. It has partially succeeded in explaining the role of change in the maintenance of the identity of an urban landscape, a point that is essential in ecological resilience dynamics. However the introduction of the concept of assemblage theory (De Landa, 2006) into place theory leads to consideration of the dichotomy between persistence and change and describes transformations in a way that makes possible a parallelism with the processes of change in an ecological resilience theoretical framework. In an assemblage all entities represent assemblages of smaller elements, "whose properties emerge from the interaction between parts" (De Landa, 2006, p. 5). Societies are assemblages of people and neighbourhoods are assemblages of certain groups of people, houses, streets and so forth. In ecological resilience socio-ecological systems are also considered assemblages of social structures and natural systems, and therefore they can be considered as dynamic, rhyzomatic structures of people and environment, in which place is both material and experiential (De Landa, 2006). 
The theory of assemblage indicates possible viewpoints of the identity of place that can be used in the analysis of the identity of urban landscapes, and particularly in the analysis of the resilience of their built environment. However, the processes that depict multiplicities, interactions of elements of assemblage, and the way the identity of an urban landscape operates in relation to change in its built environments are not explicitly defined in assemblage theory. The ways in which the built environment absorbs change and the ways in which this influences its identity and stability are still to be described. For this reason the use of ecological resilience in the analysis of dynamics of change in urban landscapes can give the opportunity to merge the philosophical concepts with ecological methodologies. This will permit an assessment and understanding of how to make the use of ecological resilience in the analysis of change in the built environment of urban landscapes effective. Finally, a philosophical approach can give more internal validation to the research, to prove that concepts in ecological resilience can be shared with other disciplines.

\subsubsection{A complex approach to the identity of an urban landscape: assemblages and multiplicities}

In the definition of ecological resilience, the concept of identity is an important attribute (see 2.21). The persistence of an identity is a condition that a system has to exhibit in order to prove that its resilience capacity was effective in absorbing changes (Holling, 1973). In the same way that identity is an important concept in ecological resilience, it is important in the definition of place given in assemblage theory. In this definition of identity, assemblage theory works as a link between philosophy and urban landscapes through the concept of place. Many of the concepts used by De Landa are based on previous works done by Deleuze and Guattari (1987). However only De Landa is directly cited in this chapter because this thesis uses his assemblage theory in order to define identity instead of using the entire theoretical construct of Deleuze and Guattari. Nonetheless, a philosophical analysis of Deleuze and Guattari might provide new contributions that can be exploited in a further research.

In assemblage theory the identity of a system is defined within the morphogenetic processes that structured the system (De Landa, 2002). Identity 
does not rely on one essential element but on the multiple relationships and elements of a system. The identity of a system emerges from changes happening through time and it is defined progressively. The concept of multiplicities offers a dynamic definition of identity that permits understanding of the change to elements and the persistence of an identity at the same time (Fig. 2-8).

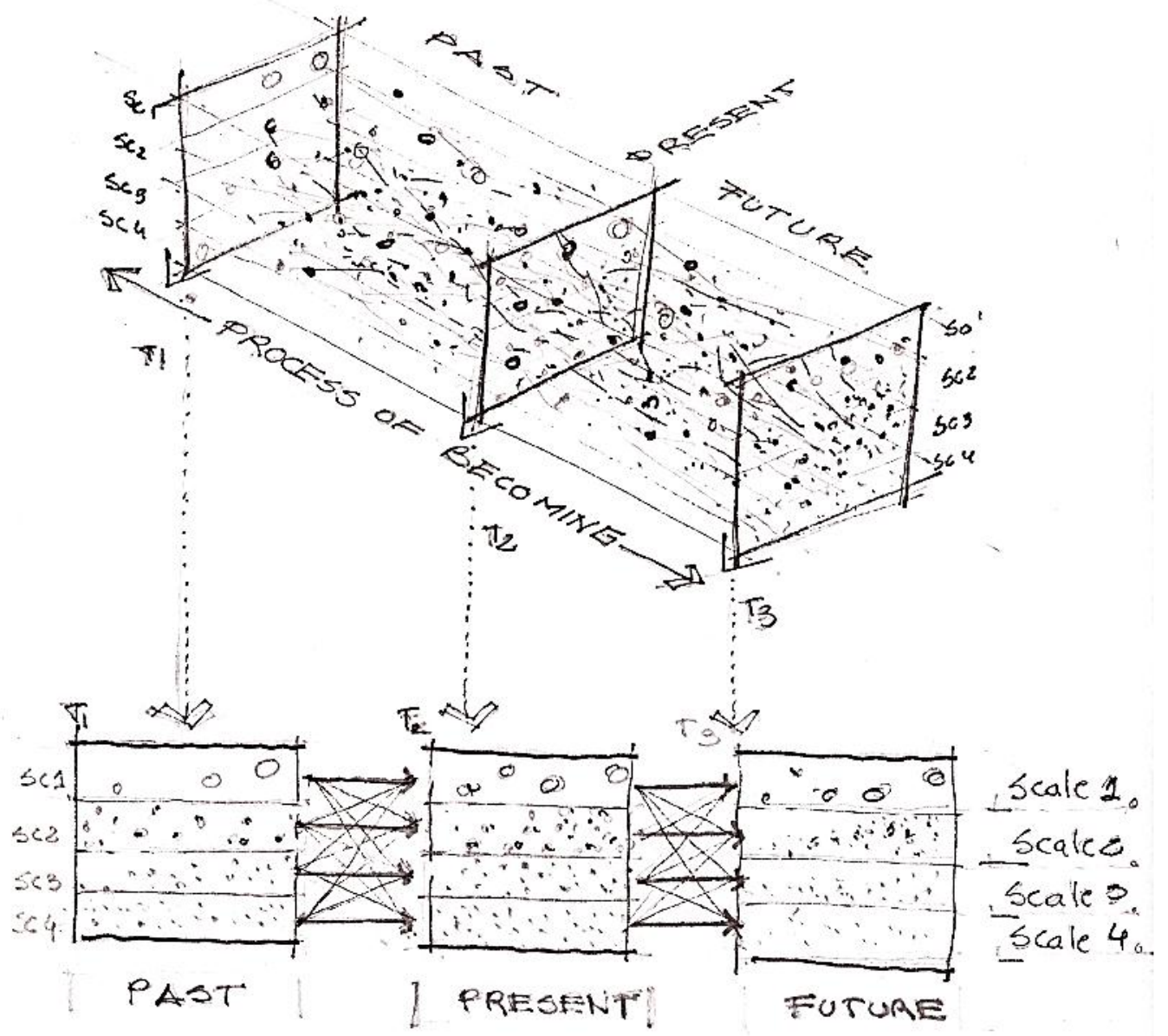

Figure 2-7. Becoming an assembled identity

Identity is an integral element of place, and as such, it is always in the process of becoming rather than a definite product (Dovey, 2010). De Landa (2006) emphasizes that identity is based upon multiplicity: "a nested set of vector fields related to each other by symmetry-breaking bifurcations, together with the distributions of attractors which define each of its embedded levels" (De Landa, 2006, p. 32). The potential of multiplicity lies in the idea that place can change and its identity can still persist. This is achieved by defining the place as assemblage; its identity is then emerging in interrelations of its parts, and appears only when the assemblage is observed as a whole. The appearance of 
identity is defined in two dimensions, material (the physical characteristics) and expressive (the qualitative characteristics and meanings), and two processes, territorialization and deterritorialization. All assemblages are a mixture of different levels of material and expressive roles. The processes of territorialization are linked with stability and homogenization. On the other side, the processes of deterritorialization imply instability and heterogenization (De Landa, 2006). Consequently, while some processes try to stabilize an identity at one level, parallel processes work in the opposite way, creating a precarious stability that comes about from the interplay of both processes (De Landa, 2006). From this point of view, the stability of an identity is related to the level of homogeneity or uniformity. In conclusion, identity always emerges from a mixture of paradoxical processes changing within and across levels (Fig. 2-8).

The concept of identity could permit characterization of a set of feedbacks among different functions and structures in urban landscapes. It can represent a way to describe how elements and structures interact in a complex system and at the same time it can be used to define how transformations affect the persistence of some elements and structures and the change of others at different levels (Garcia, Muminovic, Vale, \& Radovic, 2012).

\subsubsection{Multiple stability states and identities}

In order to explain how assemblage theory can be helpful for understanding the similarities between the dynamics of change of social systems and the complex adaptive behaviour of ecosystems (Levin, 1998) the concept of multiple stability states is explained in relationship with the concept of identity as understood in assemblage theory. This represents an approach to translating the concept of stability and multiple equilibrium points from an ecological realm to the concept of identity in a philosophical realm. The idea is to link the resilience capacity inherent in the complexity of the built environment with the dynamic of persistence and change of identity of urban landscapes, because identity represents a key point in the definition of resilience (see 2.2.1).

Change in the stability of the identity of urban landscapes and the possibility for an urban landscape to work in more than one stability state can be linked with the concept of multiplicities, as developed in assemblage, where this 
represents a number of possibilities. The concept of multiplicity is defined in assemblage theory as a number of points of attractions (De Landa, 2006) that can be directly related to the multiple stability states of complex adaptive systems (CAS). In the same way that the concept of multiplicities in assemblage theory implies different levels, in resilience thinking the identity of an urban landscape, also implies different levels of identities (Garcia et al., 2012). For example, at one particular scale, a set of relationships among structural elements defines one identity (Fig. 2-9), while at a different scale (Fig 2-10), different elements and interrelationships between them can generate a different identity (Muminovic, Garcia, Vale, \& Radovic, 2012). Even though the idea of understanding the identity of place as composite was also treated by Michel de Certeau (1984), the idea of linking the identity of place with multiple scales in space and time was not as prominent in the work of Certeau as it is in assemblage theory. In this way, identity, as shown in assemblage theory, can be understood as multiple, interacting and changing phenomena that also occur at different scales.

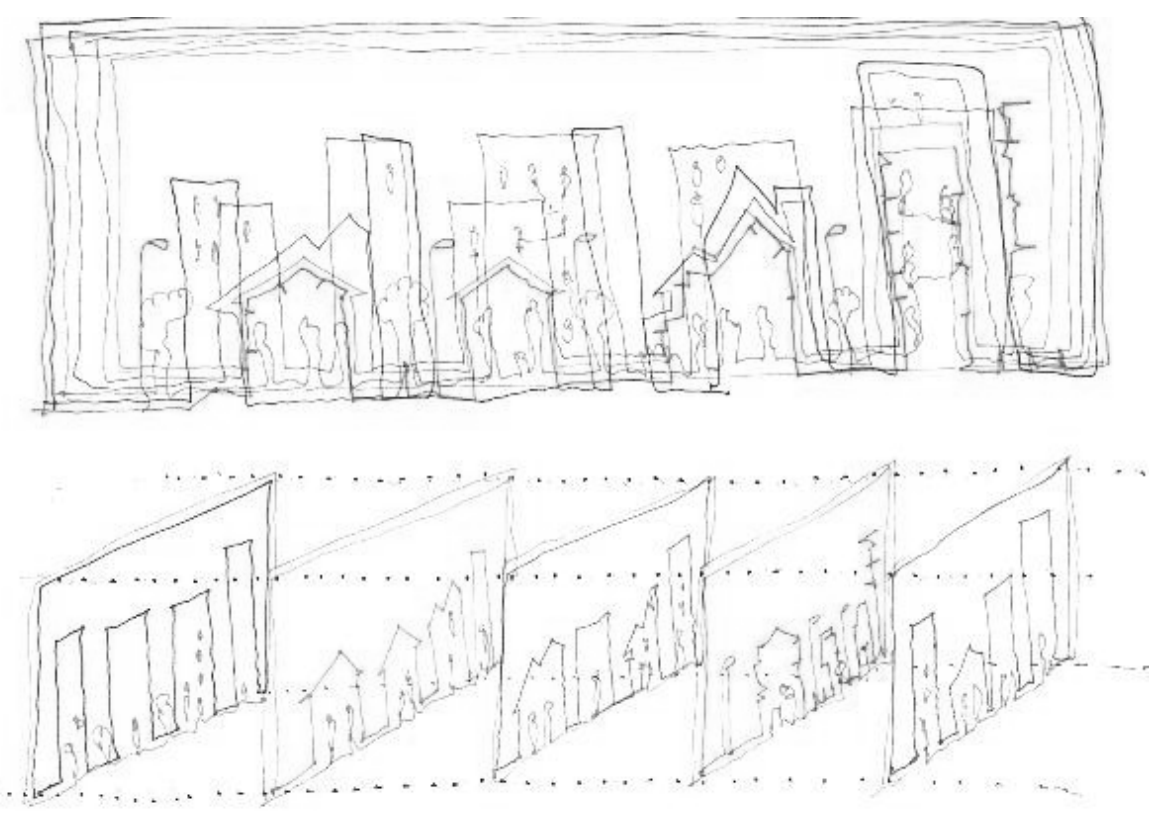

Figure 2-8. Multiplicities and identity

In the case of SES, multiplicities are related with the multiple stability states that a system can acquire at different scales (Garcia et al., 2012; 
Muminovic et al., 2012). A multiple stability state point of view implies that the set of relationships that sustain an identity are dynamic, moving and changing at different scales. This idea can be applied to the analysis of transformations in the built environment. The identity of one street, for example, could be the result of the interrelationship between buildings patterns, plot patterns, and street patterns as well as the functions and forms of these elements: "the connections between them that makes it an assemblage or a place” (Dovey, 2010, p. 16).

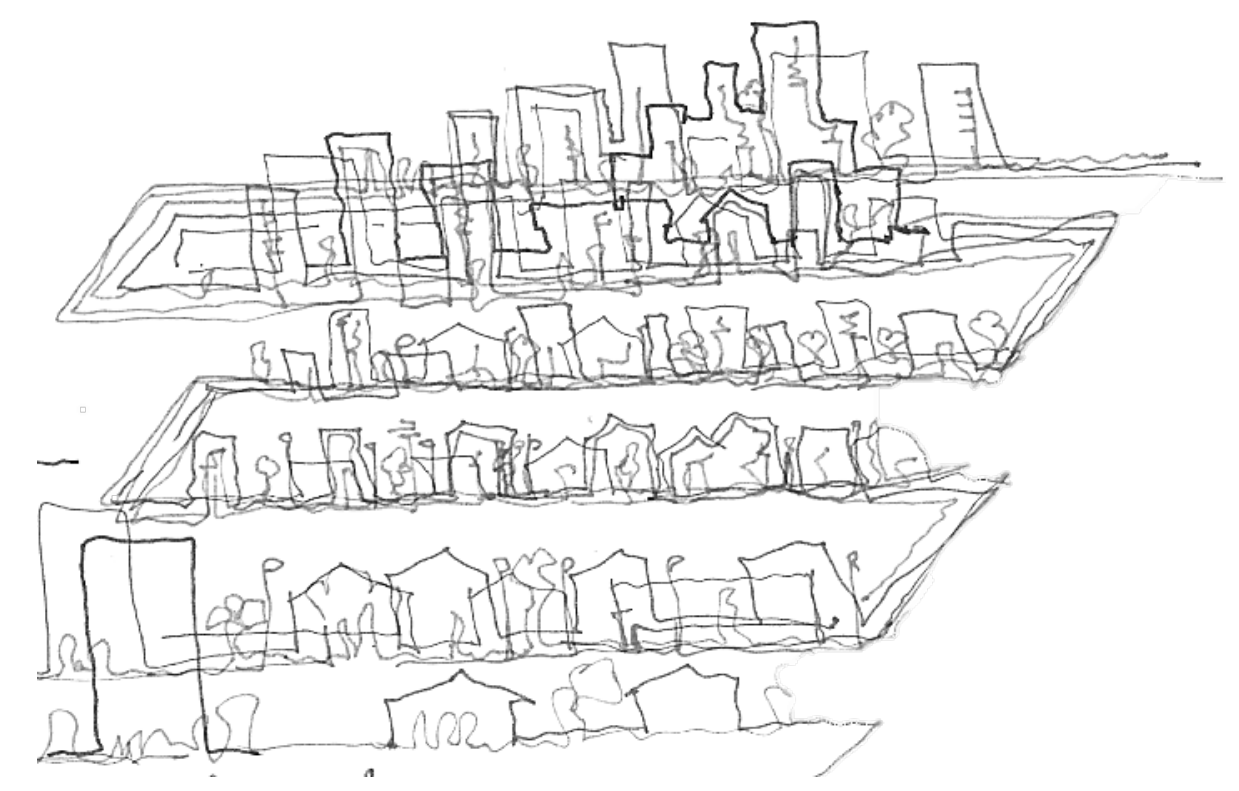

Figure 2-9. Multiplicities and multiple identities across scales

The elements can change or disappear but if the patterns that connect the elements of an urban landscape are fairly stable it will be possible to identify the street in a similar way. The identity will depend more on the evolution of the interrelationships among elements and structures in a system than transformations in particular elements by themselves (Fig. 2-10). Moreover, the persistence of an identity should imply a historical development that is not necessarily stable but is dynamic, and rarely static.

By understanding changes as symptoms of the performance and stability of an urban landscape, the analysis of morphological changes becomes important for urban design. The idea of multiple stability states challenges paradigms and notions of conservative approaches to the preservation of the identity of certain places. Urban development oriented to protecting the identity of particular 
urban landscapes can be reconsidered if they are analysed as resilient assemblages with the capacity to adapt within the inevitable forces of evolution. If it is assumed that identity is like an instant picture that describes the interplay of forces between elements and structures of the built environment of urban landscapes, within and across scales, at one moment (Fig 2-9, Fig. 2-10), the analysis of the dynamics of change becomes crucial for understanding the state of an urban system through making a diagnosis of its identity. In this manner, the understanding of the role of change in resilient assemblages, what is changing, and how it is changing, is key to enhancing or lessening the resilience capacity of built environments and to evolving the identity of an urban landscape (Table 21).

Table 2-1. Toward a resilience assemblage approach: comparative view of urban landscapes from assemblage and resilience

\begin{tabular}{|l|l|l|}
\hline & Urban landscapes as assemblage & Urban landscapes as SEL \\
\hline Organization & $\begin{array}{l}\text { Relations between sub-elements. } \\
\text { Rhizomatic and tree-like systems }\end{array}$ & $\begin{array}{l}\text { Dynamic hierarchy. Nested set of adaptive } \\
\text { cycles. }\end{array}$ \\
\hline Scale & Operate at different scales & Operate at different scales \\
\hline Causality & Non-linear & Complex and non-linear. \\
\hline Connections & Exterior and interior & Subsystems and larger systems \\
\hline Emergence & Becoming & Resilience \\
\hline Multiplicity & Set of vectors and attractions & Stability states (attractor and thresholds) \\
\hline Change & Irreversible evolutionary process & Non-linear and cyclical \\
\hline
\end{tabular}

The analysis of change, persistence, stability and resilience reveals part of the complexity involved in the investigation of urban landscapes (Garcia et al., 2012). As Morin (2001) proposed, complexity presents the challenge of thinking in a complex way in order to embrace concurrently a multidimensional understanding of the phenomena of complex and simple, and singular and general. A complex viewpoint of the identity of a built environment proposes that "its unity is not uniformity but is coherence and diversity admixed in collision" (Frazer, 1995, p. 7). The study of the complexity of urban landscapes is a requirement for research and design strategies intended to cope with the complexity that they impose. 


\subsubsection{Multiples identities assembled in a Panarchy}

Panarchy is a metaphor that synthesizes the concept of multiplicities, adaptive cycles and transformations within and across scales in such a way that is possible to understand how an identity, and therefore the equilibrium of a socio-ecological landscape, is generated in the built environment of an urban landscape in a non-linear way (Fig. 2-11). Socio-ecological systems like cities have processes happening at different scales in the landscape, affecting different quantities of space and linking with discrete temporal frequencies of change. From the system of decision in the cell of a family that is located in the building footprint of a plot and happens every day, to the groups of beliefs and traditions that were accumulated for centuries in order to consolidate the culture of a country, each sub-system has its own rate of change that affects the equilibrium and resilience capacity of the built environment at all levels. Change at the large scale is thus very slow. At the small scale of the block, as observed in the burgage cycle (see section 2.4.1), change happens much more rapidly in response to things like social forces (population increase) or socio-economic forces (increase in price of land).

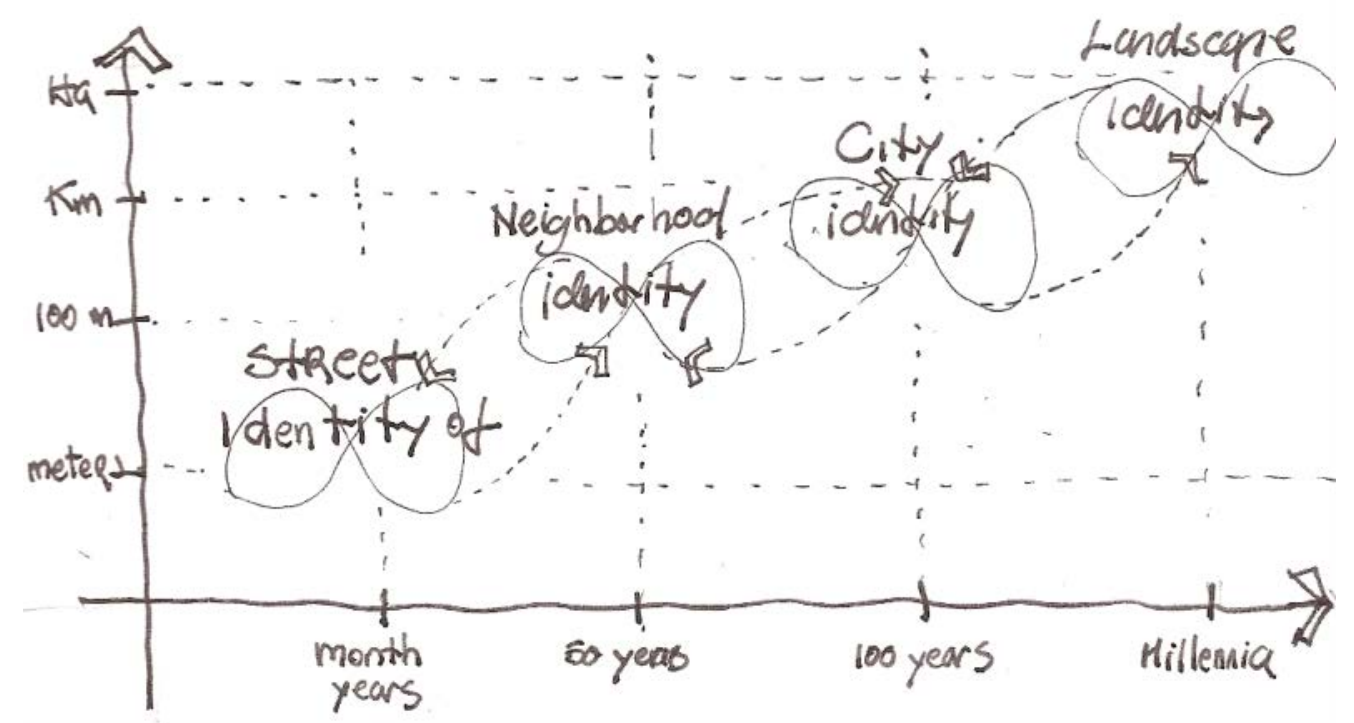

Figure 2-10. Multiples identities assembled in a Panarchy

If every scale of an urban landscape has built its own identity and this represents the equilibrium of the system, change in the identity at every scale of a system can, therefore, be linked with the loss of resilience capacities at a focal 
scale or at the level of the whole system. A resilient assemblage viewpoint using the dynamic hierarchy implied in a Panarchy can enrich the understanding of how urban landscapes change and persist by operating at different scales. Moreover, Panarchy introduces the importance of adaptive change in order to evolve and can help to explain how a system can change without losing its identity, therefore bringing essential information to the analysis of resilience dynamics. For example, the identity of a built environment that does not allow revolve can stagnate and become rigid because of a progressive loss of resilience. Systems where revolves are frequent are not stable enough to sustain an identity and therefore their equilibrium becomes more difficult to manage.

\subsection{An urban morphological approach}

If ecosystems and urban landscapes are complex systems and their adaptive processes share similar attributes, such as having a resilience capacity, both complex adaptive systems can be conceptualized and analysed using a similar theoretical approach. The theoretical framework of ecological resilience provides a methodology for analysing the evolution and the present state of an urban system while assemblage permits an understanding of similar behaviour in social systems (De Landa, 2006).

Nonetheless, the object of study is still the built environment, which means the application of ecological resilience and assemblage to urban landscapes needs a supplementary theoretical tool with which to measure change in their built environments. Because urban morphology is linked with the study of the form and shape of urban settlement (Moudon, 1997), and it is the field specialized in the understanding of changes in the urban landscape (Larkham \& Slater, 1995; Whitehand, 1981; Whitehand, 1977), it is the appropriate bridge between ecological and urban studies. In the same way that ecological resilience is trying to generate a hypothesis about the way in which ecosystems are structured and how they work, morphogenetics aims to comprehend how the urban landscape of cities originated and has evolved using town plan analysis (Whitehand, 1981). The approach is also related with the identification of key elements that structure the urban form and the contextual processes that shape urban landscapes in time and space (Whitehand, 2007). 
Because human urban systems are a complex mix of many sub-systems (Bessey, 2002), here it is argued that by looking for changes in one subsystem, the built environment, information can be revealed about the socio-ecological landscape that contains the urban landscape being analysed and in this way it can be possible to have a panorama that concerns the whole socio-ecological system.

One of the most significant works within the morphogenetic tradition was developed by the German geographer M.R.G. Conzen (1960). Conzen's philosophical approach is based in the ideal of urban landscapes co-evolving with societies and for this reason they express the development of their culture in the urban fabric (Kropf, 1993). Consequently, the analysis of the built environment allows an understanding of the uncovered processes that have shaped the urban landscape, and for this reason it is a tool for landscape management (Conzen, 1962). The underlying idea of this approach is that by mapping changes in an urban area against its ability to exhibit resilience, in other words cope with pressure without changing state, it will be possible to identify the spaces in the urban fabric that make this possible. With this knowledge and an understanding of each urban system, it should be possible to enhance or decrease the resilience capacity of socio-ecological systems to deal with unpredictable changes.

\subsubsection{Adaptive cycles in urban landscapes: the burgage cycle}

In the burgage cycle (Conzen, 1962; Whitehand, 2007), Conzen proposed a method for understanding and quantifying the evolution of urban landscapes. In the burgage cycle, the process of building in-filling in the backyards of a medieval burgage was measured at different morphological periods. Conzen found that the gradual filling up of the burgage was cyclical and that it followed different phases in relationship with discrete responses to socio-economic forces. The burgage cycle proves that the urban landscape behaves as a complex adaptive system as it follows the phases of the adaptive cycle, described above, of exploitation, conservation, release (the fallow period) and reorganisation (Conzen, 1962, p. 105). Like an ecosystem, it is also composed of discrete layers these being the sub-systems of plots, streets, and buildings. From an ecological resilience perspective it is proposed here that these sub-systems, as key 
elements of the landscape, are carrying self-organization processes in order to adapt to external demands, and therefore they behave like a complex adaptive system. The fallow periods that are produced as a result of a process of exploitation of land availability, as illustrated in the burgage cycle, could imply, from a resilience perspective, that the built environment's capacity for absorbing transformations has reached its maximum, leading to a change in state. Instead of staying in a steady saturated state, the built environment on its own and in an autonomous way is producing new spaces. It is precisely these fallow periods where the opportunities for future interventions and the possibility for further developments lie. The contention is that urban, landscape and architectural designers oriented to the prevention and mitigation of natural as well as cultural hazards have to acknowledge the assessment of these potential spaces as real opportunities to buffer massive and critical disturbances. Figure 2-12 sets out a diagrammatic representation of the burgage cycle (at the scale of the plot). It shows the need to think about urban design not as a static goal but as part of the natural evolution of urban systems. Each city is a constant series of changes happening at different scales, leading to the discussion of scales below.

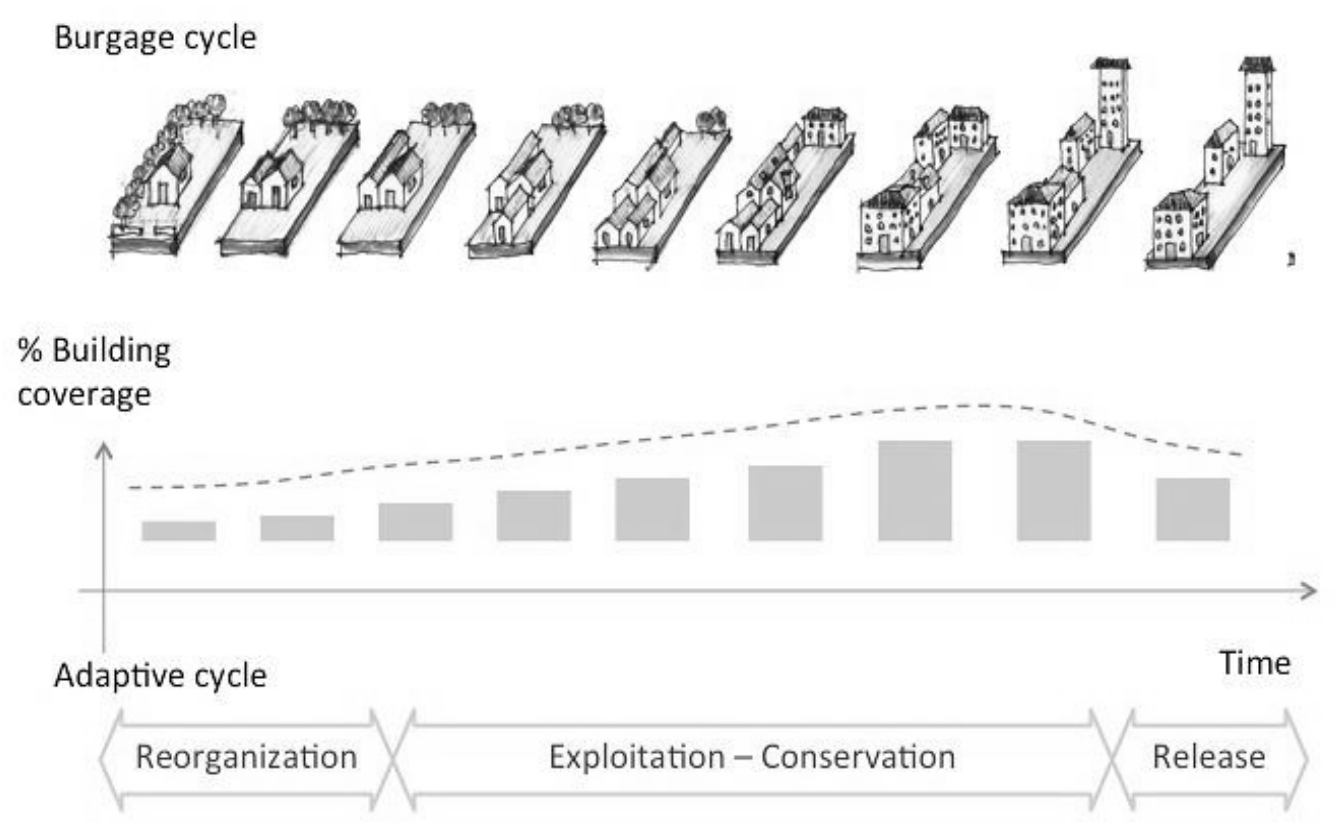

Figure 2-11. Applying the adaptive cycle to the burgage cycle 
However, there are still differences between the perspective of Conzen and a resilient assemblage approach. The Conzenian analysis implies that the urban landscape after surpassing different phases of an adaptive cycle restarts a new cycle. In the work of Conzen there is no direct consideration of the equilibrium conditions of the urban landscape as it is analysed in ecological resilience. Urban landscapes seem to work only in one possible stability state, a position that can be linked with traditional viewpoints about identity that are challenged in this research with the resilient assemblage approach (see sections 5.2 and 5.3).

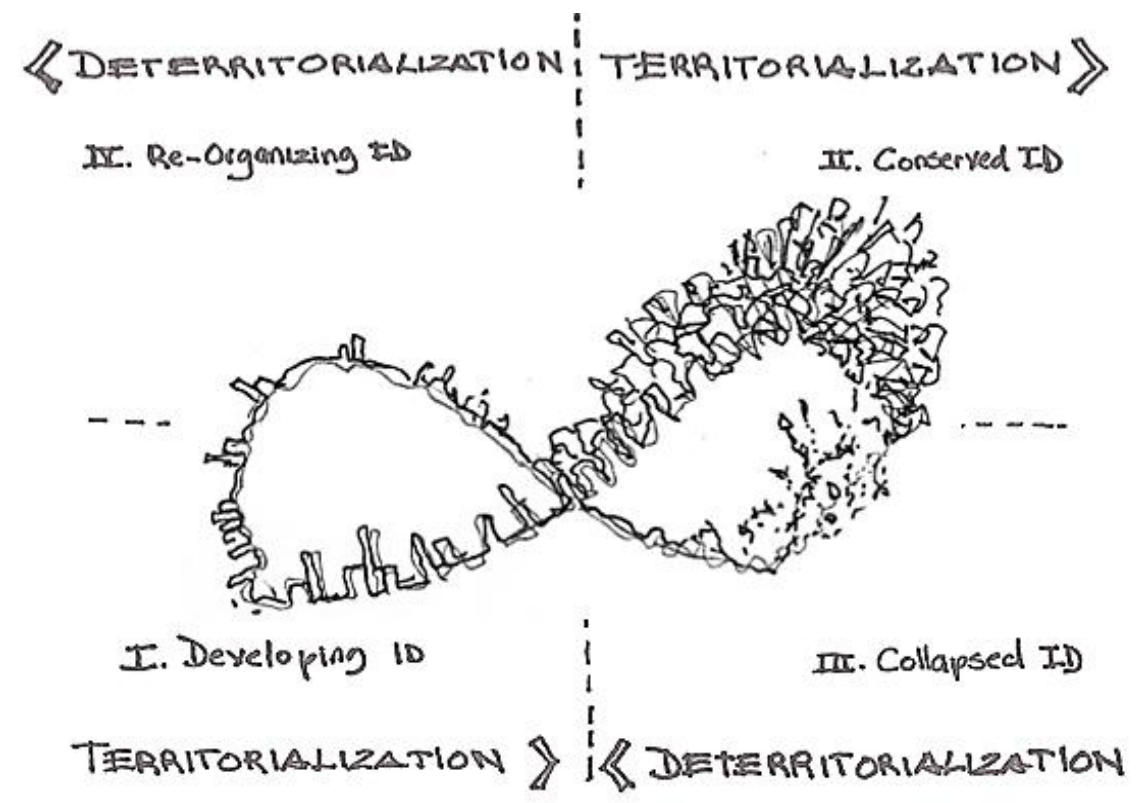

Figure 2-12. Adaptive cycle in the identity of place

Transformations in adaptive cycles could reinforce the idea that the identity of the built environment of urban landscapes is a resilient assemblage that is produced by the enduring layers of evolutionary processes that are neither linear nor totally chaotic. The identity of a complex system is pushed to adapt continuously, until the point of release of some of its elements in order to persist (Fig. 2-13).

\subsubsection{Textural-Discontinuity Hypothesis in urban landscapes}

In urbanism it is possible to reformulate this hypothesis by stating that discontinuous distribution and attributes of the elements of a built environment 
should demonstrate the existence of a hierarchical structure, which produces a discontinuous texture in the urban landscape (Holling, 1992). Discontinuities in the urban morphological and functional dimension of a landscape should produce a discontinuous SEL. If this hypothesis can be proved it would imply that complex adaptive systems, like cities, are organized in a dynamic hierarchy that can be assessed with the concepts and ideas describe in Panarchy.

The infrastructure and spaces produced at every scale of the resilient assemblage of an urban landscape should prove that elements of a socioecological landscape are not uniformly distributed but scale dependent (Bessey, 2002). Following the resilience approach, discontinuities and aggregations in the elements of an urban landscape will show the complexity of the built environment while giving the possibility of studying its adaptive qualities (see section 5.1). By analysing these discontinuities and aggregations it should be possible to show that urban landscapes are complex adaptive systems and that they are organized in a dynamic hierarchy that can be understood as a resilient assemblage. In consequence, if the TDH can be proved and consequently all the resilience dynamics of change, its use in the analysis of urban landscapes would form a first step toward the proposition of a theory of the evolution of urban form. 


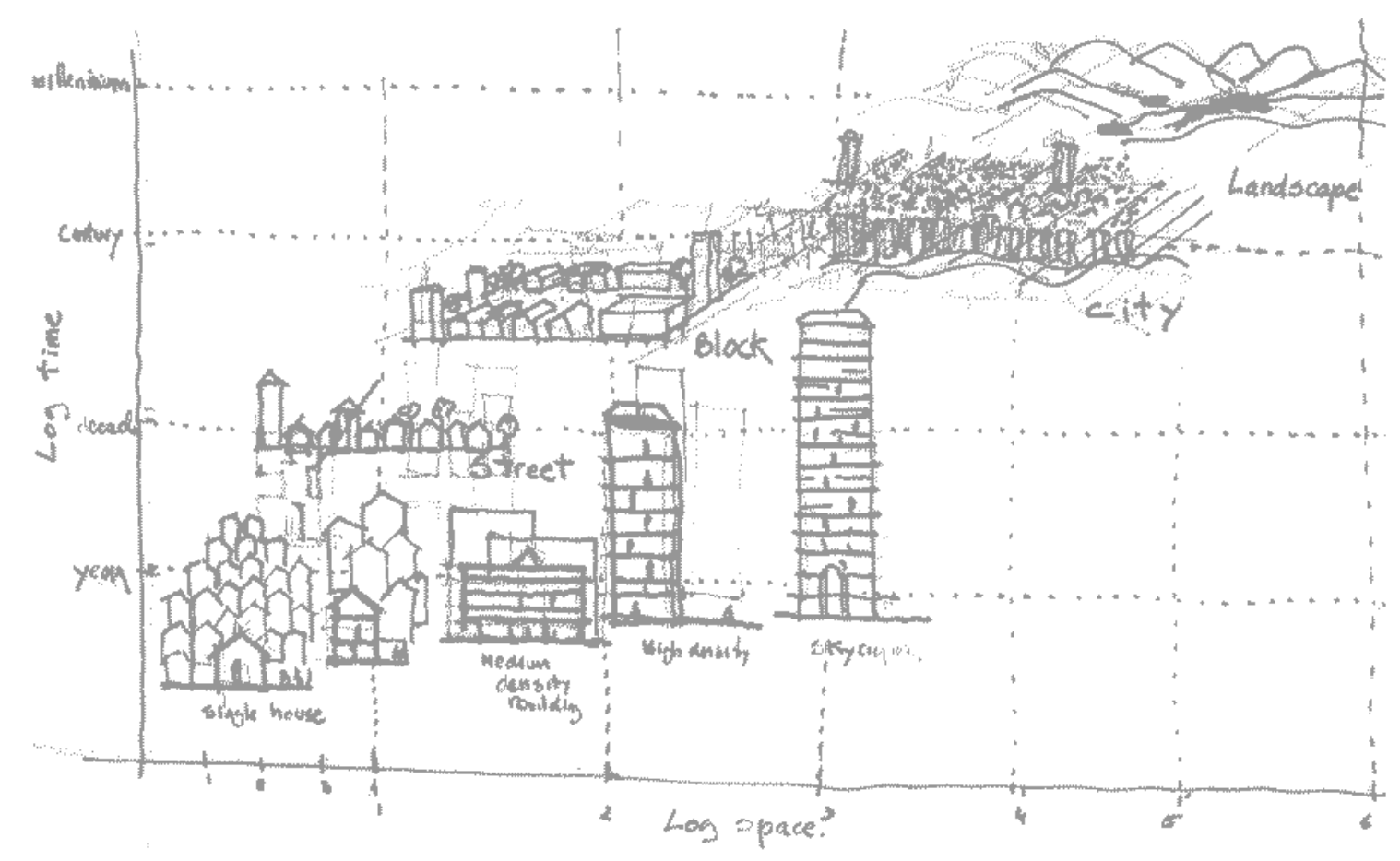

Figure 2-13. TDH in urban landscapes

SELs are landscapes hierarchically organized and discontinuous like the Panarchy described in resilience. Every structuring process of a SES (family, community, institutions, culture) produces a structuring process in a SEL (buildings, blocks, city, landscape) at different scales. The scales are defined in log time and log number of people. These habitats belong to different scales of time and space. Communities are going to produce structures at a different scale from those of families. The structures produced by communities can entrain processes from streets, neighbourhoods and cities, to regions and countries. Institutions have an impact over a wide range of scales, from the normalizations of the density and land use in one street to worldwide impact (Fig. 2-14).

The importance of the TDH for urban morphological studies lies in its potential for predicting transformations in urban landscapes by analysing changes in the geometry of the elements of the built landscape. Using morphogenetics it should be possible to aggregate mathematically key elements of the urban landscape (for example, plots, streets, buildings plans) in order to discriminate different types. For example, by grouping similar sizes of plots and buildings plans, aggregations can be produced. The result of this process will be a conceptualization of the structure of the urban landscape in terms of patterns 
of aggregations and discontinuities. These patterns will reflect the processes that originated the structure of the landscape and for this reason they can be used to compare different stability states of the system before and after a shock.

\subsubsection{Aggregations and discontinuities in urban landscapes}

Discontinuities in a database can be understood as breaks in the continuity of a sequence of variables, like missing positions in a ranking. A simple arithmetical demonstration is shown in the following example: $1,2,3,4, \ldots, 7,8,9,10$. There is a gap between the numbers 4 and 7 that discontinues the series. It is possible to aggregate a group of numbers by its similarity or its distance to a variable. In the example, the group of numbers are aggregations and the gap is a discontinuity. Aggregations are found by clustering geometric properties of different elements of an urban landscape. In urban studies the concept of aggregations has already been used in morphological analysis but only to denominate a series of building (Caniggia, 2001).

A set of aggregations and discontinuities defines a structural pattern (group of grey bars and blank spaces in Fig. 2-15). The resilience theoretical framework interprets these patterns as scales or levels of a Panarchy, where every scale is a domain of attraction within an adaptive cycle that contains information about the stability and resilience of the landscape.

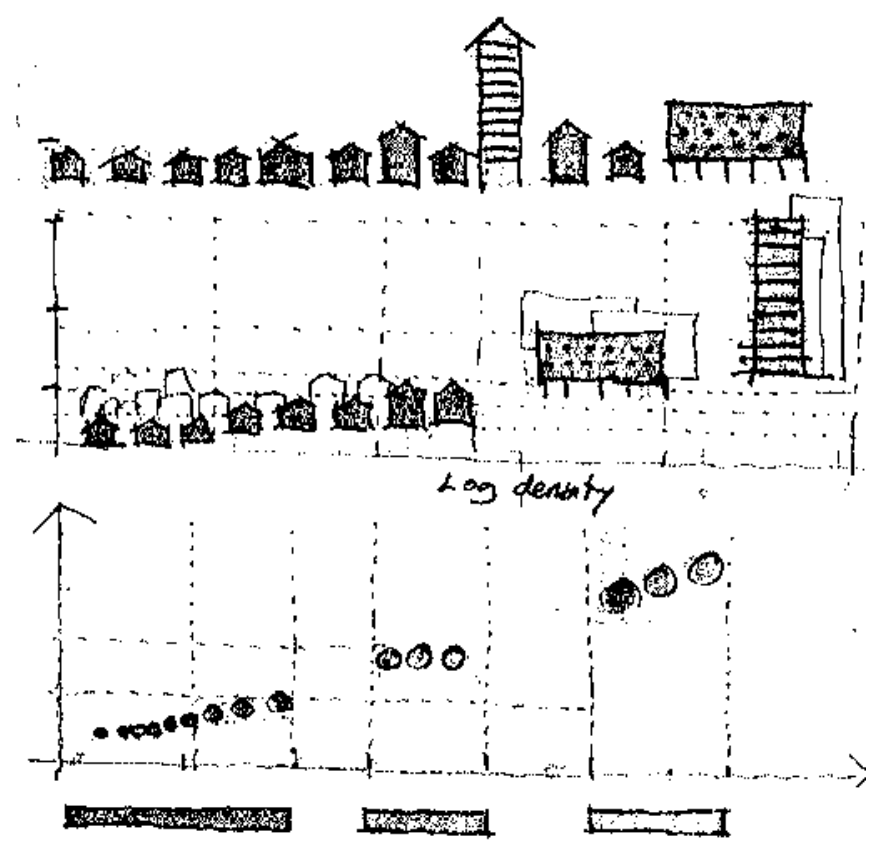


Figure 2-14. Aggregations and discontinuities in urban landscapes

Figure 2-15 illustrates the process of identification of discontinuities and aggregations using a morphological analysis. In this example, elements of the built environment of a SEL are grouped into typologies and densities. As a result it is possible to obtain different aggregations separated by discontinuities between scales. The final result is a pattern of three aggregations (grey bars) and two discontinuities (blank space between grey bars). In urban studies the idea of discontinuities can be used in many different ways because it can be linked with the lack of continuity, the non-linearity and the intervals contained in every single social or natural process in a city.

Discontinuities can be linked with interruptions in the continuity of a variable, these being, for example, the absence of elements in landscape. For example, blocks are not normally the size of a house (building footprint). The absence of blocks of house size causes a discontinuity between the group of elements that are the size of a house, and that conform to an aggregation of house sizes, and the ones with the size of blocks, that generate an aggregation of elements of the size of a block. In this case a discontinuity implies a change of scale between the aggregation of building footprints (the size of a house) and the aggregation of the areas of blocks.

Discontinuities can be related in a metaphorical way with other concepts like "terrain vagues", "loose spaces" or "interstitial spaces" (Lévesque, 2009; SolàMorales i Rubió, 1995). The definition of terrain vague given by Sola-Morales (1995) alludes to an empty and abandoned space. The architect declares that these spaces are a sort of negative space. They are elements that generate the systems of folded and interstitial spaces creating the "residual city" (Solà-Morales i Rubió, 1995, pp. 120-121). Levesque (2009) analyses the concept of terrain vague as an interstitial space that represents the contradictions between modern society and its environment. From this point of view, the terrain vague becomes a material with which to build an open city, characterized by the amalgam of heterogeneous components. The approach of looking at the terrain vague as interstitial spaces or fragments that result from the clash between the 
developments of social systems within a natural system is an idea that can be directly linked with the dynamics of interaction in socio-ecological landscapes as described in the theoretical framework of ecological resilience. The implication of the thoughts of Levesque is that the analysis of the origin of interstitial spaces is connected with the comprehension of societies and the environment as a single system.

The difference between the concept of discontinuities in ecological resilience (C. Allen \& Holling, 2008) and these in-between spaces is that discontinuities in a resilience approach are not necessarily associated with a kind of space but with the lack of intensity in a particular morphological process. Discontinuities in the built environment of a SEL can also be understood as the result of the complexity and heterogeneity of a resilient assemblage. Discontinuities are the product of the process of transformations generated by the evolution of the morphology of a built environment.

\subsection{Discontinuity and heterogeneity}

In ecological resilience a Panarchy produces dynamic and hierarchical landscapes that are self-organized by clustering resources discontinuously (C. Allen \& Holling, 2008; Holling, 1992). As a result a lumpy landscape is formed by aggregations and discontinuities. In urban landscapes it could be possible to think about the heterogeneity of a built environment as a variable that can be explained in relationship with the complex dynamics of change happening in a Panarchy. If urban landscapes are organized as a Panarchy, as proposed in the hypothesis, then it could be possible to think that the heterogeneity of an urban landscape is linked with the complexity, diversity and resilience capacity of the system.

In order to analyse the heterogeneity and discontinuity of urban landscapes it is necessary to consider not only elements that are built, but also those that are not built. The reason for considering un-built spaces as important elements in the analysis of the resilience of urban landscapes is that they constitute physical discontinuities in the built environment per se. They can be understood as a reserve of available space and therefore a resource for the future of urban landscapes. From this point of view, un-built spaces could be 
playing an important role in the control and self-organization process of urban landscapes. Moreover, a network of un-built spaces may be related with the buffering processes of change happening at interfaces in the built landscape.

\subsubsection{Discontinuities and public-private interfaces.}

The analysis of interfaces in urban landscapes is important for the recognition of identity and complexity. Interfaces are areas for encounters between systems, where the major quantity of information and interchanges take place (Pesci, 2007). In ecology and ecological resilience, interfaces can be considered as areas of transition that contribute to the diversity, adaptability, and evolution of complex systems (C. Allen \& Holling, 2010; Lewin, 1999; Turner, Davidson-Hunt, \& O'Flaherty, 2003). Interfaces in complex adaptive landscapes are areas at the edge between order and chaos (Kauffman, 1993) where resources are volatile and species have to develop the adaptive capacity to cope with unpredictable changes and scarcity of resources (C. Allen et al., 1999). Therefore, interfaces become excellent places to test the resilience of a system because they are linked with discontinuities in the urban landscape and, perhaps, therefore form a path to explore the idea of spaces for resilience. In this thesis interfaces were linked with two kinds of spaces: private-public interfaces and green spaces.

Public private interfaces were chosen as a subject of study with the possibility of linking these with interfaces, loose spaces and spaces for resilience due to their importance as transitions in the urban landscape. Public-private interfaces represent intersections between the public and the private realm in an urban landscape. Sidewalks or streets can be understood as zones that represent discontinuities in the built environment. In consequence their analysis is linked with the diversity and complexity that together may enhance the resilience of urban landscapes.

\subsubsection{Discontinuities and green spaces.}

The importance of and necessity for green spaces in cities is a fact that has been extensively researched in urban sustainability, landscape ecology and architecture (Higueras, 2006; Leal del Castillo, 2004; Moisset \& Paris, 2005; Tzoulas et al., 2007). Most literature about green spaces in urban landscapes is 
mainly focused on the impact that urbanization has had in the fragmentation and depletion of green spaces (L. Walker, 1999). Although investigations have been conducted to analyse the importance of the general role of green spaces in providing opportunities and adaptability in urban landscapes (Colding, 2007; Elmqvist et al., 2004) and landscape architecture (Allan \& Bryant, 2011), analysis and measurement of the role of green spaces in the resilience of the built environment of urban landscapes has not been fully developed.

It is possible to think that green spaces are not just the reverse of the built environment but that they have their own structures and produce their own adaptive capacities. In this way they should provide diversity for the whole structure of an urban landscape while exhibiting a resilience capacity to changes in its built environment. It is believed that green spaces contribute to the diversity and discontinuity of urban landscapes because they physically represent interfaces between layers (scales) within the built environment. In this way, green spaces can be understood as the zone of encounters between natural and social systems in a city. Even though green spaces in a city are under the dominion and control of the social part of a system, they still represent the natural system and a reserve of land resources that can be used in the case of an emergency. Hence, the history of green spaces could have the potential to describe the quantity of interactions between elements of the assemblage in an urban landscape. If it is possible to understand green spaces as part of a network of inter-spaces that are associated with discontinuities, they should be related with the production of diversity within and across scales in a system. The analysis of the role of green space in the dynamics of change of the built environment can, therefore, produce important information about the way built environments change, adapt and use their resilience capacities to keep on evolving while maintaining their structures in a fairly stable condition.

\subsubsection{Spaces for resilience}

The dynamics of change and adaptation processes between built and nonbuilt spaces presented in interfaces is an excellent opportunity for the development of a research path towards the discovery and definition of a present and future network of spaces for resilience in urban landscapes. Spaces 
for resilience are opportunities created within the evolution of urban landscapes. They are the system of spaces that emerges from the interaction of all the past and present interventions in the urban landscape within and across different scales in the urban system. Basically what are being identified are spaces of opportunity, these being a potential of the urban landscape that needs to be considered in the present to make development in the future possible. Such spaces are not only the interstitial group of spaces suggested by Sola-Morales (1995) or Gilles Clement (2007), but also the opportunities these spaces generate when they interact with the pre-existing landscape.

Rather than seeing spaces for resilience as a theoretical construct, this thesis argues that they emerge naturally as the non-linear response of the urban system, but that their form is dependent on the structure of the particular urban system. In this manner they become an essential element of the urban landscape that can be better observed in the evolution of the particular landscape. This leaves the question as to whether it is possible, through identification of spaces for resilience, to be able to design for them as part of increasing the resilience of the socio-ecological system and its urban landscape. For this reason it is important to investigate this set of emergent spaces as well as their interaction with the entire socio-ecological system using resilience thinking (Garcia \& Vale, 2012). The importance of this issue for a city and particularly for architects, landscape architects and urban designers, is that the existence and emergence of a set of autonomous spaces in the built environment can be associated with the appearance of opportunities to receive new uses and to provide alternative solutions in the face of present and future threats.

\subsection{Conclusion}

Ecological Resilience can offer a comprehensive approach for investigating changes in urban landscapes in order to understand their capacity to adapt and to deal with unexpected disturbances.

The evolutionary process presented in the behaviour of ecosystems can be compared with the dynamics of change presented in urban landscapes because both are complex adaptive systems. As was shown in this chapter, the theoretical framework of ecological resilience can be a suitable way to analyse 
and to rethink cities in order to produce a better understanding of the resilience and stability of urban landscapes. This knowledge can generate an alternative way to analyse and comprehend the design of urban landscapes. The benefits that can be obtained from a resilience approach can be synthesized in the following points:

1) Stability and resilience are attributes of complex systems, like ecosystems and cities, which define their capacity to adapt and to evolve.

2) The understanding of the reality and quality of urban landscapes can be achieved in relationship with an understanding of equilibrium in which multiple stability states are possible.

3) The resilience of an urban landscape depends on general and specific processes and responses that occur in the system, within and across scales.

The present epistemological approach produces an interface of knowledge that makes every key concept more accessible for its application in urban studies.

The morphogenetics of Conzen provides the syntaxes of the urban landscape. Some of the findings in this chapter are related with the common fields and possible future contributions between ecological resilience and urban morphology as well as with the possibility of assessing the resilience of urban landscapes using morphogenetics. As was shown in the burgage cycle, the built environment of a SEL is not just a consumer of socio-ecological services but also a generator of spaces that can serve for buffering change. For this reason the built environment becomes an essential element in analysing the resilience of cities.

Ecological resilience explains that social and ecological systems are in constant interchange. In an evolutionary approach, change is neither good nor bad per se. Change has to be evaluated in a wider perspective, considering the specific and general performance of a system within and across scales, before and after shocks.

Ecological resilience and morphogenetics are concerned with the evolution of socio-ecological systems and landscapes. The understanding of cyclical change as a pattern of evolutionary processes is another common subject. However, in ecological resilience processes are non-linear and the cycles 
can be affected by shifts. Shifts introduce an alternative understanding of urban landscapes in terms of different levels of stability co-existing and interacting.

Resilience and an assemblage viewpoint highlight the importance of analysing the situation of an urban landscape not only in terms of aesthetic attributes, historical heritage or social interaction, but also in terms of the probabilities of its adapting to new challenges while continuously evolving. Resilience introduces the importance of designing urban strategies with an emphasis on producing the necessary adjustments that an urban landscape needs in order to survive and persist.

Ecological resilience offers a theoretical framework which can be used to assess change as a way of understanding how a system adapts in order to evolve. Through the analysis and comprehension of change it is possible to assess the adaptive capacity of a system. The analysis of the quality and quantity of these changes is linked with the stability and resilience of a system to face disturbances. From this point of view, a retrospective analysis of the evolution of transformations in a built environment can be utilized to infer the present adaptive capacity of an urban landscape, and it can also be used to create alternative scenarios for the landscape in order for it to confront future hazards.

The analysis of change, persistence, stability and resilience reveals part of the complexity involved in the investigation of urban landscapes (Garcia et al., 2012). As Morin (2001) proposed, complexity presents the challenge of thinking in a complex way in order to embrace concurrently a multidimensional understanding of the phenomena of complex and simple, and singular and general. A complex viewpoint of the identity of a built environment proposes that "its unity is not uniformity but is coherence and diversity admixed in collision" (Frazer, 1995, p. 7). The study of the complexity of urban landscapes is a requirement for research and design strategies intended to cope with the complexity that they impose.

The structural and functional diversity of an urban landscape could help to produce and maintain discontinuities that represent opportunities when the system has to face unpredictable disturbances. 


\section{Criteria for assessing the ecological resilience of urban landscapes}

\subsection{Introduction}

The aim of this chapter is to describe the most important references that inspire and make possible the translation and application of concepts from ecology to the morphology of urban landscapes. The chapter has three elements: methodologies, applications in urban studies, and the generation of an episteme that permits the methodology to be applicable to urban landscapes. The content is arranged in two groups. The first group deals with principles and criteria with which to assess the dynamics of change in urban landscapes. The second group is focused on explaining how to use discontinuities and aggregations to assess resilience in urban landscapes.

According to the theoretical framework of ecological resilience the adaptive capacity of an ecological landscape can be assessed by analysing the geometric (Holling, 1992) and functional (Peterson et al., 1998) characteristics of elements present in the texture of that landscape (see section 2.4). If similar criteria are applied to urban landscapes, it should be possible to quantify changes in the morphology of the built environment as a way of assessing its resilience and stability (Garcia, Vale, \& Allan, 2011). The assumption made is that the form, rate and speed of changes in the built environment produce urban landscapes that exhibit a particular structure and stability. The stability state and performance of the urban landscape are linked with the resilience of the system (E. Garcia et al., 2012). Therefore alterations in the stability of an urban landscape can be used to measure resilience in urban landscapes.

The Resilience Alliance (2007a) has developed an integrative methodology with which to assess the resilience of socio-ecological systems. The specific aim of this methodology is the recognition of thresholds and the measuring of changes in relationship with those thresholds. In this chapter the methodology of the Resilience Alliance is supplemented with the analysis and measurement of the relative resilience of systems by using the assessment of 
discontinuities in order to measure the functional richness, heterogeneity and diversity of a system (C. Allen, Gunderson, \& Johnson, 2005). This analysis is important because "distribution of functional diversity within and across scales enables regeneration and renewal to occur following ecological disruption over a wide range of scales" (Peterson et al., 1998, p. 1). What is at stake in the analysis of socio-ecological landscapes is the capacity that urban landscapes, and especially their built environments, have to produce more or fewer opportunities for present and future developments in the same urban landscape.

\subsection{Assessing the dynamics of change in analysing the ecological resilience of urban landscapes}

\subsubsection{Limiting and bounding a system}

In order to analyse the evolution of urban landscapes, the Resilience Alliance in their methodology suggest first defining the system that will be analysed and also its context. A system in this case could be the urban landscape of a city, a neighbourhood, or just one block, and the relationship of each one with the whole system. Considering that in resilience thinking (Walker \& Salt, 2006) it is essential to be aware of the multiple equilibrium behaviour of a system, the boundaries of the system to be analysed along with its context need to be studied as moving variables in a process of change, or as variables in a process of becoming (Garcia et al., 2012). In the theoretical framework of ecological resilience (Resilience Alliance, 2007a) the limits of a system are defined in relationship to scales of time and space, along with the domains of each subsystem involved in the process of change being analysed (Holling, 1992). The boundary of a system is therefore related with the scale of the issue affecting the system.

In urban landscapes, issues are multiples and they are all linked with multiple scales, a factor that increases the complexity of defining the scale of the issue affecting particular urban processes of change. The boundary of a contextual issue in the built environment of an urban landscape can be approached by analysing the history of its economic, cultural or environmental development, but this will not provide the same quantity and quality of information that can be found in ecological studies, where a system can be 
bounded and analysed by considering fewer subsystems. It is in this step of trying to bound a system where the important concept of identity and the observation of changes in the identity of an urban landscape can be used to describe the situation of a system. The concept of identity when it is linked with the stability state of an urban landscape can be a parameter for inferring that a system is still keeping the same set of relationship with its context (see section 2.3.2). Therefore the absence of changes in the identity of an urban landscape can be related with the persistence of the same boundary and the relevance of the same issues.

\subsubsection{What are the main issues affecting the system?}

The resilience theoretical framework suggests analysing the key components of the context of a system, such as the economic, institutional, ecological and social domains (Resilience Alliance, 2007a). The reasons for choosing and creating only these subsystems are not specifically sustained in the methodology of the Resilience Alliance and there has been criticism of this (Hornborg, 2009; Jerneck \& Olsson, 2008). However, if the analysis of urban landscapes were done considering the variables proposed in table 3-1, it would still be a very challenging task. The amount of data achieved will be very complex to manage and sometimes it will be hard to find the amount and diversity of information required for different scales and different periods. However, despite these criticisms, the framework provided by the Resilience Alliance is still a good starting point that can be supplemented or changed, depending on the type of analysis to be performed.

Table 3-1. Possible domains of analysis in ecological resilience (Resiliance Alliance, 2007)

\begin{tabular}{|l|l|l|l|l|}
\hline $\begin{array}{l}\text { The natural } \\
\text { resources }\end{array}$ & $\begin{array}{l}\text { Institutional } \\
\text { charge of the } \\
\text { preservation or } \\
\text { conservation } \\
\text { uses of the } \\
\text { natural } \\
\text { resource } \\
\text { analysed. }\end{array}$ & $\begin{array}{l}\text { Social } \\
\text { (community/society). } \\
\text { Community viability } \\
\text { is a variable of } \\
\text { concern in such an } \\
\text { SES, influenced by } \\
\text { what is happening in } \\
\text { the biophysical part of } \\
\text { the system. }\end{array}$ & $\begin{array}{l}\text { Ecological } \\
\text { ecosystem goods } \\
\text { and services. For } \\
\text { example, clean } \\
\text { water and soil } \\
\text { fertility } \\
\text { maintenance. }\end{array}$ & $\begin{array}{l}\text { Ecosystem goods } \\
\text { and services that } \\
\text { are directly used. } \\
\text { Economic } \\
\text { (commercial } \\
\text { crops, timber, } \\
\text { tourism, etc.), } \\
\text { subsistence } \\
\text { recreational, } \\
\text { aesthetic, cultural } \\
\text { (tradition, ritual), } \\
\text { conservation. }\end{array}$ \\
& & & & \\
\end{tabular}




\begin{tabular}{|l|l|l|l|l|}
\hline The people & $\begin{array}{l}\text { Individuals and } \\
\text { organizations } \\
\text { that manage } \\
\text { and use } \\
\text { resources. }\end{array}$ & $\begin{array}{l}\text { Conflicts between and } \\
\text { points of agreement } \\
\text { between groups. }\end{array}$ & $\begin{array}{l}\text { Values to manage } \\
\text { natural resources. } \\
\text { Learning and } \\
\text { innovation. }\end{array}$ & $\begin{array}{l}\text { Economic status of } \\
\text { people. } \\
\text { Wealthy/poor. } \\
\text { Limitations for } \\
\text { lack of financial } \\
\text { resources. }\end{array}$ \\
\hline Governance & $\begin{array}{l}\text { Key policies } \\
\text { laws and } \\
\text { regulations. }\end{array}$ & $\begin{array}{l}\text { Local norms and } \\
\text { taboos. }\end{array}$ & $\begin{array}{l}\text { Organizations that } \\
\text { control the } \\
\text { resources. }\end{array}$ & $\begin{array}{l}\text { Property rights. } \\
\text { Public land, } \\
\text { private land, } \\
\text { common land or } \\
\text { other form of } \\
\text { property. }\end{array}$ \\
\hline
\end{tabular}

The economic, institutional, social, philosophical, cultural or political components of the context of systems not only produce the situation and boundaries of a system but they are also changing and this affects their rules, demands, viewpoints, and management. The present research assumes that socio-ecological landscapes are created by the interaction of all social and ecological systems. Urban landscapes are one subsystem contained in a socioecological landscape. Consequently, it will be possible to state that the main issues affecting the dynamics of change of urban landscapes are all subsystems of a socio-ecological system.

\subsubsection{The resilience of what to what}

In order to define which subsystem of an urban landscape and which dimension of its context are to be analysed it is important to define "the resilience of what to what" (Carpenter et al., 2001) is being analysed; in other words, what variables are going to be compared? This comparison could be made in order to understand the resilience of a system before and after a disturbance or in order to analyse its overall adaptability through a period of time. An example of bounding a system using this approach could be the resilience of the density of a neighbourhood to changes in its land prices.

The idea behind the logic of the resilience of what to what is being analysed is that the adaptive capacity of a system is linked with being adaptable to something else. For this reason the assessment of the resilience of a system has to be carefully attended by limiting its scope in order to clarify the resilience of what to what is being analysed. A resilience analysis will always imply a comparison between a system that is analysed and its context. In urban studies, the analysis of the resilience of what to what can be very broad and susceptible 
to different interpretations, depending on the focus of analysis chosen by the scholar. For example, what is at stake in the description of the work of Conzen (see section 2.3.5) can be formulated as the resilience capacity of the built environment of urban landscapes to fluctuations of cultural context, in space and time, and more specifically the resilience of the town plan elements of building footprints, plots, and streets to fluctuations in socio-economic factors.

\subsubsection{Scales}

In ecological resilience, the concept of scale is a central topic that is linked with the way of bounding a system and defining which domains and issues should be considered at which level of resolution (Resiliance Alliance, 2007a). " $A$ domain of scale is the spatial extent of a structure or process of interest and its temporal frequency" (C. Allen \& Holling, 2010, p. 1). By framing key processes from a group of previously selected domains (such as ecology, social, institutional) and questioning the resilience of what to what is being analysed, it is possible to narrow down the research regarding urban processes of interest and urban structures using a resilience approach. However one challenge that the ecological concept of scale presents when trying to apply it in the study of urban landscapes is the understanding of the frequency of change of each urban process or structure. This knowledge involves research into a deeper understanding of scale and an investigation about the rate of change in time and space of every urban process or structure and its extent and resolution in terms of space.

The concept of scale has a different meaning and implications depending on the field of research that uses it. Scales are essential in urban investigations that aim to analyse the relationship between patterns, like urban types, tissues or frames, and processes, for example processes of change in the built environment. Scale is defined in this thesis as an analytical dimension (spatial, temporal) that is required to assess or to investigate a phenomenon. It is possible to distinguish three primary dimensions of scale: space, time, and organizational level. The extent, size of the dimension analysed and its grain, the unity of the measurement, are important concepts that can be used as tools to circumscribe and define the scale of an urban phenomenon. Finally, it is possible 
to conceive that different extents in space and time are linked at different levels, forming different types of hierarchies (Gibson, Ostrom, \& Ahn, 2000; Wu \& Li, 2006). All these concepts can help the analysis to be more accurate when scale framing, and using concepts from morphogentics (see section 2.2.3) in the analysis of the resilience of urban landscapes.

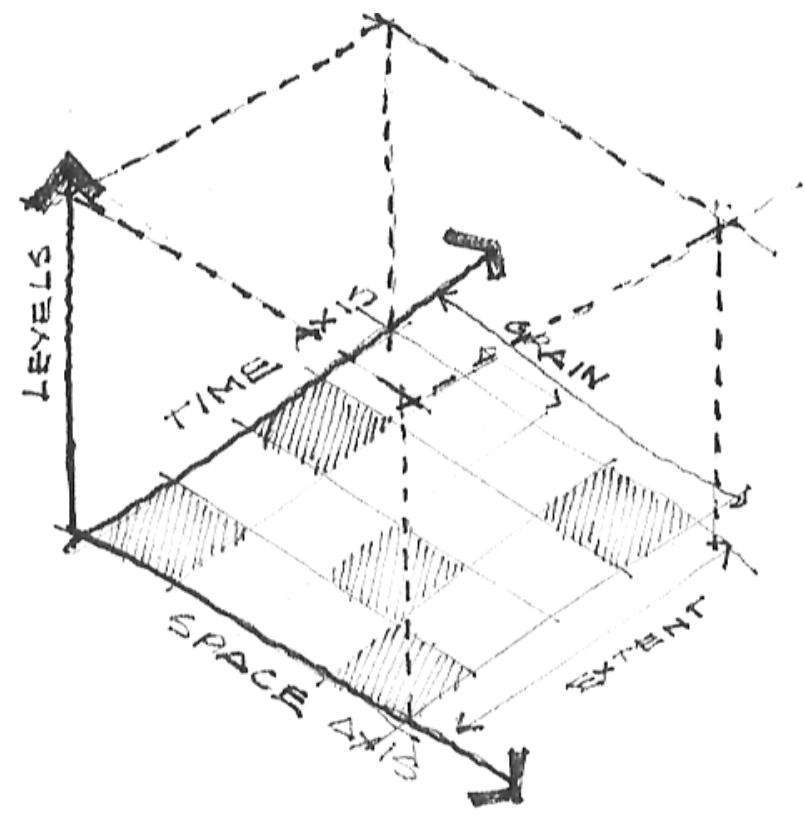

Figure 3-1. Scale dimensions based on (Gibson et al., 2000; Wu \& Li, 2006)

\subsubsection{Scale mismatches and scale framing}

The concept of scale in urban systems presents a challenge due to its complexity. From a resilience viewpoint, socio-ecological landscapes are the assemblage of multiple processes in social and ecological dimensions. Socioecological systems have different frequencies of change, at different scales, and dynamics that produce different boundaries in relationship with the discrete scales in the system. The overlap between different processes and boundaries at discrete scales produces mismatches that complicate the definition of the boundaries of each subsystem (Gibson et al., 2000). For example, the definition of the geo-political boundaries for a territory may not match the conservation area set up to protect certain ecological processes. In order to try to transcend these mismatches in the analysis of socio-ecological landscapes, the concept of scale framing can be used. Scale framing is a theoretical approach that highlights 
one aspect of a reality in order to circumscribe a problematic that needs to be analyzed (van Lieshout M., Dewulf, \& Aarts, 2011). The concept of scale becomes broader allowing linkage between different processes and subsystems, like identity frames or power frames. Hence, scales prove to be a theoretical tool for analysing complex systems and increasing the understanding of how they work and are organized. In the study of the resilience of urban landscapes, all concepts related with scales and scale framing have to be developed at a different level in order to assemble interdisciplinary fields, at different levels, while establishing connections with the processes of change in the built environment occurring at discrete extents and grains in the urban landscape. But, which criteria can be used to frame the scale of processes occurring in urban landscapes?

\subsubsection{Multiple scales}

The work of Braudel $(1969,1977,1980)$ is important in finding a set of principles to frame events and processes at different scales. The ideas proposed by the French historian (Braudel, 1980, p. 4) will be used as a reference to define temporal frequencies in urban landscapes. Particularly, this will help in defining the scales of analysis suitable for analysing social and ecological systems; what should be the content and extent of every scale and what events and processes have to be placed at which scales in socio-ecological systems?

The global understanding of history developed by the French scholar produced a collision between social sciences and history, generating a social history of nature, or a history of the appropriation of nature by different societies. This work was a significant contribution to the theoretical foundations of environmental history (Padua, 2010). Braudel argues in favour of recognizing and understanding the evolution of social systems in relationship with ecological systems (Hexter, 1979). The resilience concept of socio-ecological systems is based on a similar principle which links socio and ecological systems (see section 1.4).

The methodology that Braudel used to construct a global vision of history relies on a multi-scale analysis of the history of a territory. The milestone of his work is the emphasis on the history of a problem as the unit of analysis instead of a region. The application of the theoretical framework of ecological resilience 
to the analysis of urban landscapes also deals with the issue of linking problems and regions coherently. This approach can be linked with the idea of scale framing already mentioned.

Braudel proposed three scales based on the duration of historical processes: the longue (long), moyenne (intermediate), and court (short) duree. These speeds are related with three conceptions of time: a "geographical time, a social time, and an individual time" (Braudel, 1980, p. 4). The geographical time tells the history of man and the relationships with his environment. It is a history of slow changes that take place in cycles, where structures are produced as well as trends that exceed the control of human beings, for example, long-term variants in climate. In urban studies this scale could be linked with slow processes of big transformations at the level of landscapes and regions. Its measurement could be expressed in thousands of years and thousands of kilometres. The social time is the history of social realities, which is constituted by "all major forms of collective life" (Braudel, 1980, p. 11) like economies, societies, and demographic changes that follow cycles of ten, twenty-five, or at the limit fifty years. This scale can be approached in urban studies by measuring its processes in decades or hundreds of years (Wegener et al., 1986). Finally, the history of events is the history of individuals. This history is characterized by fast and powerful changes, and is a micro-history. This history could be measured in days, weeks, or years (Wegener et al., 1986).

Braudel defines three scales of time that represent three histories that are independent but interconnected at the same time, as in the concept of Panarchy in ecological resilience or in the concept of constitutive hierarchy. By using the concept of duree he additionally sketches boundaries for problems based on the frequency of change of those processes. The next challenge is to link these discontinuities and multiplicities in time with correlative processes in space, and particularly in the built environment of urban landscapes, as proposed in Panarchy. 


\subsubsection{Timeline: assessing change in urban landscapes by identifying cycles and phases}

A plausible step in assessing the complexity of city systems and urban systems is found in the analysis of their evolutionary processes. Analysis of the evolution of urban systems permits the mapping of the history of their adaptations. The potential contribution of this approach is an increased chance their management will be more successful leading to the improvement of cities.

A timeline should provide information about events and critical breakpoints that have shocked a system as well as information about the possible reasons that triggered the change. Radical changes, collapses, and crises, can be symptoms of a break in the capacity of a system to withstand internal or external pressures. A timeline will help in describing the performance and evolution of a system and in defining parameters for setting the thresholds of systems, which means stating how much is too much for that system. Therefore the resilience analysis of the evolution of urban landscapes through timelines can reveal how social priorities and ecological systems vary through time. This method allows the discovery of which processes and elements were driving and defining the character of a system through time.

\subsubsection{Method for producing timelines}

In order to generate a timeline for understanding resilience dynamics it is first important to identify the scale of analysis and domains, as explained previously. The theoretical framework of ecological resilience suggests analysing key variables at 3 to 5 different scales of time and space (Resilience Alliance, 2007a; Holling, 1992). The first step to producing a timeline consists of creating parallel lines, where each one is documenting the evolution of discrete domains (economic, ecological) at different scales. The second step consists of placing along each timeline major events that have perturbed the system. It is recommended the temptation of trying to fill in all the boxes is avoided (Resilience Alliance, 2007a). The idea is just to outline the break points and try to make connections between domains and scales. The third step consists of characterizing periods before and after a disturbance, for example by conceptualizing every era with different names. The investigation requires 
listing possible causes that have made a system vulnerable to a triggering event. The last step consists of looking for possible patterns of change, like the frequency of disturbances, scales and domain in which they have happened with more intensity, and diffusion of their impacts, which means identifying the direction of the responses, for example bottom up or top down. The output of this examination should be the definition of the critical domains of the system and its patterns within and across scales.

\subsubsection{Understanding disturbance and collapse in cyclical change}

In ecological resilience the different phases of an adaptive cycle (see section 2.3.4) are described in relationship to fluctuations observed in the capital and connectivity of a system. Variations in capital and connectivity are related with the stability and resilience of the system. The breaking point of the metaphor is the release phase, which represents the collapse of the variables analysed and the starting point of a new loop in the system. Therefore the definition of shocks, crises, collapse or releases becomes important for situating a system in its cycle of adaptations and also to understanding either alterations or shifts to a different stability state. In the methodology of the Resilience Alliance (2007a), a disturbance is defined as any alteration to a system. Natural disasters, economic crises, and revolutions are all examples of disturbances. However the concept of collapse is not clearly defined.

In order to assess cycles of change in urban processes one challenge is to define and differentiate when a system is experiencing a disturbance and when it has collapsed. For these purposes the work of Tainter (1988) will be used. Also a historian, Tainter developed a general explanation of collapse based on his analysis of the downfall of complex societies. He defines the collapse of a society as a socio-political matter with an impact on multiple subsystems. A collapse is "a rapid, significant loss of an established level of sociopolitical complexity" (Tainter, 1988, p. 4). In this definition complexity implies not only the quantity of variables, function and interactions observed in a system but also their interrelationships with two qualities: inequality and heterogeneity. Inequality is associated with the difference in levels of a social structure where the lowest levels have less access to resources. Heterogeneity is linked with the variety and 
diversity in a society. Tainter describes the level of complexity of a society using as an indicator the level of complexity that the political state of a society has achieved. Even though it would be interesting to investigate if complex states produce more complex urban landscapes this is not the goal of this research. However it could be possible to use the concept of changes in the complexity of a system, for example change in the complexity of the built environment of urban landscapes, to discover thresholds and to measure the resilience of a system in relationship to those limits. Making a parallel with the definition of Tainter, a socio-ecological system might absorb as many unpredictable changes as its socio-political structure might tolerate.

Tainter, by using the concept of heterogeneity and inequity, explains that a society evolves from being less complex to being more complex when its growth produces a differentiation and specialization of its parts while multiplying its levels of control, with the consequence of providing less equal access to resources. The hypothesis of Tainter is that societies in order to keep on growing become very efficient in the development of problem solving systems that can absorb the deficit that they produce in their processes of growth. A collapse emerges when the difference between the cost of becoming more complex and the cost of maintaining the complexity already acquired arrives at a breaking point where negative marginal returns cannot be handled (Fig. 3-2). The result is the loss of complexity of the system. This impacts through the atomization of the structure, provoking less centralized control, diminishing specialization, less density, and fragmentation. 


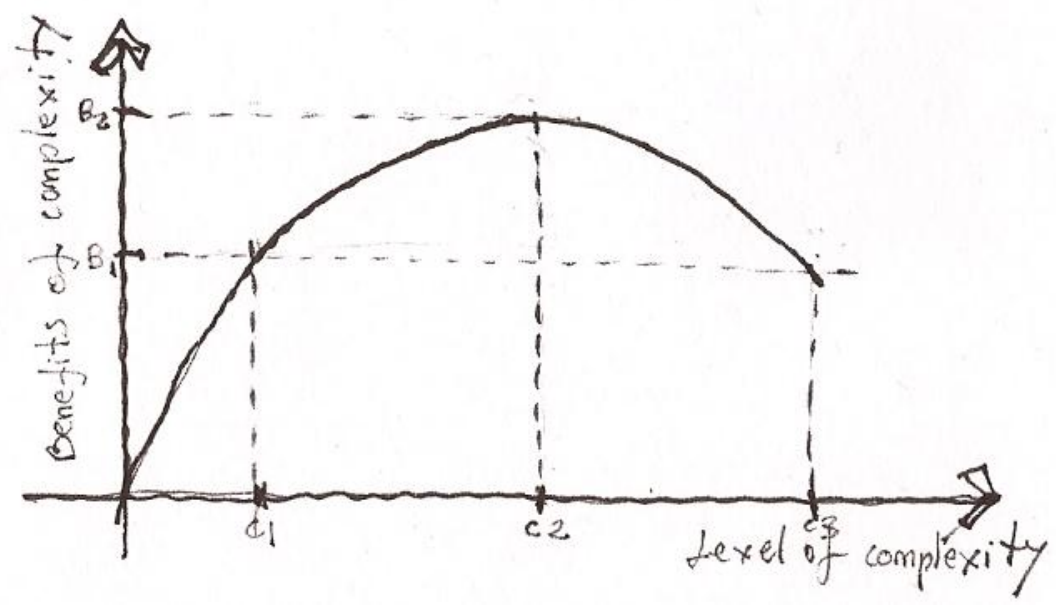

Figure 3-2. Diagram of the cost-benefits of complexity (Tainter, 2000). B1, B2, C1, C2, C3 are only references to imply different amounts of complexity and benefits of that complexity.

It is possible to link the definition of collapse from Tainter with the processes of change in the resilience theory. The dynamics of change proposed in the metaphor of the adaptive cycle states that the pass from an exploitation phase to a conservation phase, where a system becomes more complex before it collapses, is characterized by specialization, more control of resource consumption and the distribution of capital between fewer groups. This reflects the way that complex societies, or ecosystems after the release phase or collapse, reduce complexity leading to the capital being redistributed more evenly.

In the analysis of socio-ecological systems it would be possible to say that Tainter's analysis could be useful for identifying when a system has collapsed and differentiating this point from a disturbance. The challenge then is to find a way to describe and quantify the degrees of complexity in a system, particularly in the built environment of urban landscapes, the object of study of this thesis. Another important criterion to be developed is the assessment of how fast a change means a rapid loss, and how much is a significant diminishment in any level of complexity. Measuring fluctuations of capital can assess disturbances and collapses. In any case, the dynamics of change of elements of an urban system in space and time will be linked with the capacity of a complex system to selforganize in such a way that the levels of complexity achieved do not change dramatically after disturbances, particularly within its diversity and heterogeneity. 


\subsubsection{Constructing and evaluating an urban Panarchy}

Urban designers, managers and researchers deal with great level of complexity when trying to assess the dynamics of change in urban landscapes due to the multiple scales involved in all urban processes (Gibson et al., 2000). The way in which every scale of the system works and how larger scales affect smaller scales and vice versa, becomes a compulsory step in the analysis of the built environment of urban landscapes. In ecosystems, larger processes are slow and smaller ones are fast. However in an urban system this rationality is challenged. Small elements of an urban system, like houses, that affect small quantities of territory can change less frequently than governments or other forms of management, that affect larger extents at different scales through their top down decisions.

The construction of a hypothetical urban Panarchy is carried out by applying the analysis and diagnosis described in the methodology. The benefit of building a Panarchy is the understanding of the overall performance of a system. This performance can be assessed by comparing change in the structure and function of an urban landscape at different scales in space and time. The evaluation of the behaviour of the Panarchy of an urban landscape can basically produce two scenarios. In the first, due to its resilience capacity, the system is adapting by buffering changes within and across scales; in the second, the resilience capacity of the system to buffer disturbances within and across scales has been exceeded, therefore the system has collapsed and it is starting a new

phase in a different stability state (B. Walker \& Meyers, 2004). The diagram presented in Fig. 3-3 is a synthesis of these concepts. 


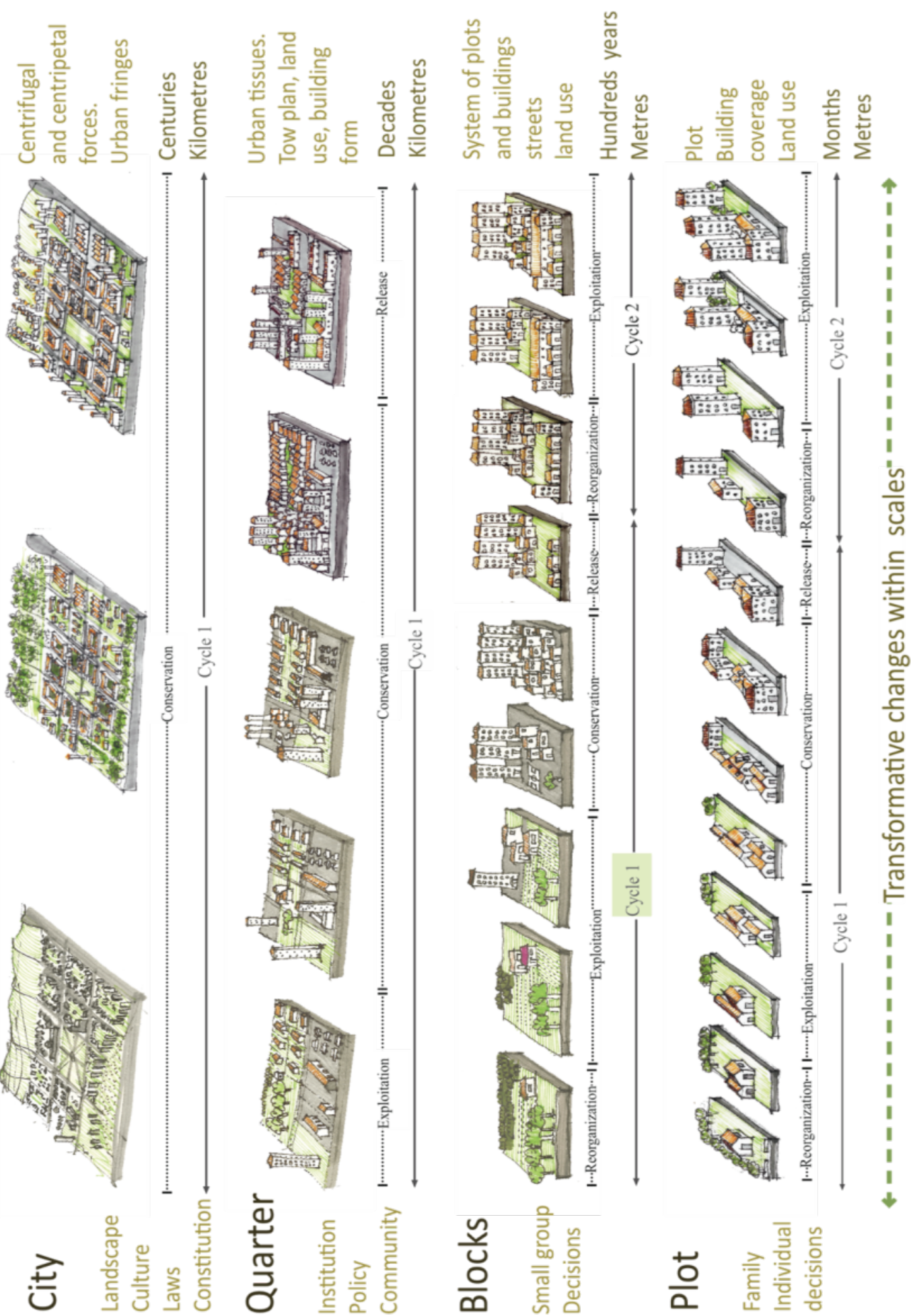

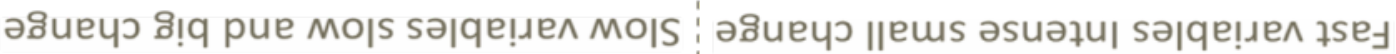

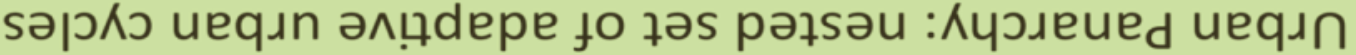

Figure 3-3. A hypothetical urban Panarchy 


\subsubsection{From urban morphology to an urban Panarchy}

Cities are very conservative structures that do not change much at the large scale but that do exhibit a diversity of speed of change at smaller scales. The resilience approach to these results is that the dynamism of a multi-scale structure along with the difference in speed of change is precisely what gives resilience and stability to the urban landscape at bigger scales (Bessey, 2002; Garmestani et al., 2005). One important difference between urban models that are more or less dynamic (Wegener et al., 1986) and the knowledge developed in this research is that in a resilience approach, processes, people, physical structure, and nature are linked and assembled in one socio-ecological landscape and are part of one urban Panarchy (see section 2.3.6). In this way, a Braudelian approach seems to be important because it links societies and nature in one analysis. The construction of an urban Panarchy can contribute to the production of a theoretical framework to be used or developed in future urban models.

In urban morphology the complexity of the morphology of the built environment is crucial and helpful for designers, however the concept of scale is still fuzzy. The morphogenetics of Conzen (see section 2.2.3) in contradiction with the work of Braudel, used geographical units that arose from the town plan as different levels of resolution and grain and that helped in analysing change in urban landscapes. The concept of scale, not generally discussed by Conzen, could be linked with the level of resolution at which change in space and time of elements is presented in the urban landscape. However, it can also be understood as the extent of the structures created by these elements when they are grouped at different levels. In one way or another, the result is that the urban landscape is a whole constituted by an arrangement of parts (Kropf, 1993) with three dimensions, namely town plan, land utilization and building fabric. Conzen's focus is on the analysis of town plans, where he identified three key elements: street, plots and block plan. Each element when clustered produces a subsystem called a complex, namely street systems, plot patterns, and building patterns. Combinations of these complexes of elements produce plan-units, aggregations of plan units, and generate plan-divisions. The assembled town plan, land utilization, and building fabric patterns generate a morphogenetic region. The cross scale relationships between all units of the urban landscape, at 
each historical socio-economic stage of development, produce morphological periods (Kropf, 1993).

A reasonable criticism of Conzen from a resilience approach could be that these morphological periods are linked with traditional essentialist viewpoints of identity, linear ways of understanding change, and the prominence of socioeconomic factors as drivers of change but without considering the noncoherences, produced by bottom-up forces and self-organization processes. These also produce changes not necessarily aligned with historical periods and are therefore difficult to put boundaries around. From a resilient assemblage approach, and using the elements defined by Conzen, it is possible to propose that the social and ecological processes happening in streets, blocks, and buildings create links within and across scales in time and space that establish more or less temporary structures. The difference between both philosophical lines is the way of understanding and characterizing urban landscapes. In morphogenetics, urban landscapes are a collection of final forms, for example morphological periods are final forms resulting from a particular historical period. In a resilient assemblage strategic view urban landscapes are complex systems, an assemblage of micro and macro structures where connections and elements are still in constant evolution. A Conzenian vision characterizes final forms in urban landscapes while a resilient assemblage approach temporarily assesses structures of the urban landscape as diagrams.

A resilience approach to the analysis of urban landscapes should consider an intersection between Braudel and Conzen in order to rethink scales in a sufficiently complex way to allow the integration of the social and ecological realms, towards an understanding of urban landscapes as socio-ecological landscapes. Conzen proposed that town plan, buildings, and land use have different resistances to change; for example, land use and building fabric are more susceptible to change than town plans, and street systems are more resistant to change than plots and building plans. These sets of relationships between form and time permit the inference of rates of change that are very important when proposing complexes of elements as spatio-temporal scales of the landscape. By scale framing social and ecological processes within the extent of the plots, streets, and building footprint patterns it will be possible to 
associate socio-ecological processes with different spatio-temporal scales, simplifying the process of choosing the right scale or morphological period for analysis. This idea was not explicit in the work of Conzen; however, it could be a very useful complement to his work by introducing the scale factor independently of the extent and dominion of morphological regions.

In an urban Panarchy, this hierarchy of discrete resistances to change can be related with different spatio-temporal scales in relationship to slow and fast variables. In this case, streets are slow variables that contribute to the stability of the landscape while land use is a fast variable that leaves room for the introduction of opportunities.

\subsection{Assessing resilience through the analysis of discontinuities: the urban Panarchy}

The identification and characterization of phases, disturbances and collapse are essentials steps in assessing adaptive cycles and therefore in having a better understanding of the dynamics of change of the system. The next step is to analyse what is changing or has changed in the structure of a system, at precise scales, before and after a disturbance. This kind of analysis will give more information about the relative resilience of the urban system. In order to do this it is useful to describe the stability of the system before and after a shock.

Assuming that ecosystems are structured in a Panarchy, driven by a few sets of processes working at different scales and producing discontinuities in structures and landscapes, the persistence or change of these discontinuities will reveal changes in the structure of the system. In this case discontinuities are expressed in scale breaks that constitute the different levels of the Panarchy and also in the internal constitution of every scale. If the structure of an urban landscape is characterized by different levels, namely, building footprints, blocks, plots and streets and after a disturbance streets disappear, the structure of the system will experience a loss of one level in the structure of its urban Panarchy, lessening its complexity and changing its organization. In the example the loss of one discontinuity in the scale organization implies that the system could not buffer the loss of streets, therefore, it is possible to infer that the resilience capacity of the built environment was surpassed, due to the lack of self- 
organization or richness, ending in a shift in the identity of the urban landscape. Consequently, the generation and persistence of discontinuities and aggregations determine the way in which a system is self-organized (C. Allen et al., 2005). The self-organizing dynamics of complex systems will try to support the location of discontinuities even though some elements of the subsystems disappear. The Panarchy will always try to maintain the system in a fairly stable situation unless the resilience of the system is exceeded. For all these reasons, the persistence of discontinuities might be a characteristic of the resilience of complex systems. A resilient system would be the one that can maintain a pattern of functions even though some elements of the systems change or get lost (C. Allen et al., 2005). Ecological studies confirm the importance of functional richness within and across scales for the resilience of a system. These studies show that resilience was higher in ecosystems that maintain redundancy of function within and across scales (Peterson et al., 1998). Even though the observations were made in ecosystems, the conclusions that they obtained should be applicable to the structures of all complex adaptive systems, like cities (P. Allen, 1997).

According to all these facts, the conclusion is that measurement of resilience can be assessed by analysing change in the organization of discontinuities and aggregations. Changes in patterns of aggregations and discontinuities, within and across scales, can be used to quantify the resilience of complex adaptive systems (C. Allen et al., 2005), like the built environment of urban landscapes.

\subsection{The use of Panarchy and discontinuities in urban studies}

An interesting example of the importance of using discontinuities for measuring functional richness in order to understand resilience was performed in economics. The research makes a parallel between the functional richness of species in ecosystems with the necessity for achieving functional richness across firms in urban systems. The research observed how employment volatility in industries varies in relationship to their functional richness. Results found that measurements of the volatility of employment in firms can be used to assess the resilience capacity of an economic system. Industries with a diverse functional richness across and within scales experience lower rates of turnover and more 
resilience than industries with less functional richness (Garmestani, C. Allen, Mittelstaedt, Stow, \& Ward, 2006).

Bessey (2002) proposes that there are similarities between landscapes produced by ecosystems and those produced by urban systems. Ecological and urban landscapes show spatial patterns hierarchically structured and scale dependent. He suggests that these patterns reveal structural resilience because they can keep their structure and function after disturbances, due to buffering dynamics across scales (Bessey, 2002). Bessey affirms that urban systems present temporal and spatial discontinuities in their configurations, for example, in discrete city growth rates and clustering in city size distribution. The analysis of regional urban systems in the USA shows that the frequency of growth rates at a city scale does not define the path of growth at a national or regional level. At big scales, regional urban systems tend to concentrate population in larger cities. Meanwhile, at smaller scales the evolution of cities happens in intervals of stability and instability which are entrained by regional and national processes (Bessey, 2002). This is basically the same dynamic of change already explained in the adaptive cycles and Panarchy. The whole urban system has the capacity to absorb some degree of disturbances originating at lower scales, while remaining fairly stable at overall scales (Bessey, 2002).

The hypothesis and results obtained by Bessey were used in other investigations related to the use of discontinuities and time series analysis in order to determine city size distributions (Garmestani et al., 2005; Garmestani et al., 2008). These studies were also based on the idea that urban systems are discontinuous and hierarchically structured as explained in the proposal of an urban Panarchy. The result is that the production of discontinuities between city size classes is a demonstration of the existence of discrete scales. The structuring processes at each scale entrain city size classes with similar characteristics into groups or aggregations. These city size classes exhibit a stability state at their respective scales. Different city size classes produce different scales in the systems, an observation which proves that the urban system can hold multiple stability states (Garmestani et al., 2008). The results suggest that cities operate like complex adaptive systems and that resilience theory might be a useful tool in the study of urban systems. 
All these studies were done analysing urban systems at city scales but there is still a gap in the translation of ecological resilience to its application in the scale where changes in the built environment matter. For architects, urban and landscape designers the information is useful but is still far from the scale of analysis related with everyday life and the scale where transformations in the built environment are critical.

Considering the previous research discussed here it should be possible to propose that the stability state of an urban landscape can be assessed by observing changes in its structure and functional diversity using discontinuities and aggregations as objects of analysis. From this point of view, the structure of an urban landscape can be abstracted into a set of aggregations and discontinuities.

\subsection{Finding aggregations and discontinuities in urban landscapes}

In an urban morphological approach, aggregations can be found in the different levels that the morphology of the built environment contains, for example the different scales constituted by buildings plans, plots, and streets. The objective of aggregating values is to find the quantity of groups of information with similar sizes existing in a database. The idea is to simplify the management of large databases by reducing the whole information into a few groups, for example groups of big, medium and small elements. Detailed mathematical explanations are not the purpose of this thesis considering that there is a variety of applications that deal with calculations to perform multivariate analysis, cluster analysis, and data mining processes, like Weka (Hall et al., 2009; Witten, Frank, \& Hall, 2011), Orange (Curk et al., 2005), or R (Hornik, 2012). However a description of the criteria chosen to make the cluster analysis in this thesis is described below.

Cluster analyses in this thesis were performed in chapters 5 and 6 using Weka (Waikato Environment for Knowledge Analysis) version 3.7.7. Calculations were done using the "explorer" interface because it was the more appropriate for non-experts. The data introduced was imported from corresponding excel tables. The data was collected from digitisations of buildings footprints, plots, blocks and streets done in QGIS and ArcGIS, as explained in the data collection section 
(see section 1.12). The data introduced were areas $\left(\mathrm{m}^{2}\right)$ of building footprints, plots and blocks. The data was imported to Weka from the excel tables using a csv format (comma separated values). Each table or data set contains all features corresponding to building footprints, plots or blocks areas in a certain year. Then the data is processed by using a selected clusterer method. Clusterer options were tested until results satisfactory for the purpose of the research were achieved.

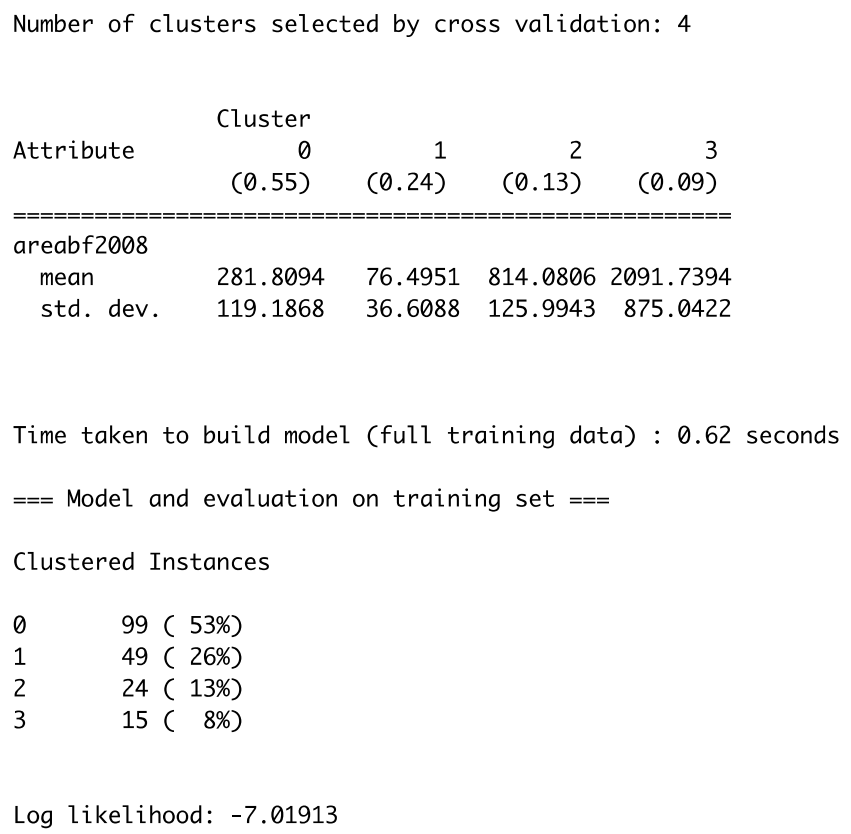

\section{Figure 3-4. Typical cluster analysis report}

In figure 3-4 the cluster analysis was done using the data of all the building footprints areas from 2008 in the east side of Auckland CBD. Calculations were done using the training set option in Weka with the simple EM (expected maximization) class EM -I 100 -N -1 -M 1.0E-6 $-\mathrm{S}$ 100, where maximum alterations equal 100, min. standard deviation equals $1.0 \mathrm{E}-6$, the number of cluster equals -1 , and the seed equals 100 . Then clusterer outputs were collected and visualized. In figure 3-5 the visualization of the example in figure 3-4 is presented. Every cluster found has a different colour that identifies the elements belonging to its cluster class. 


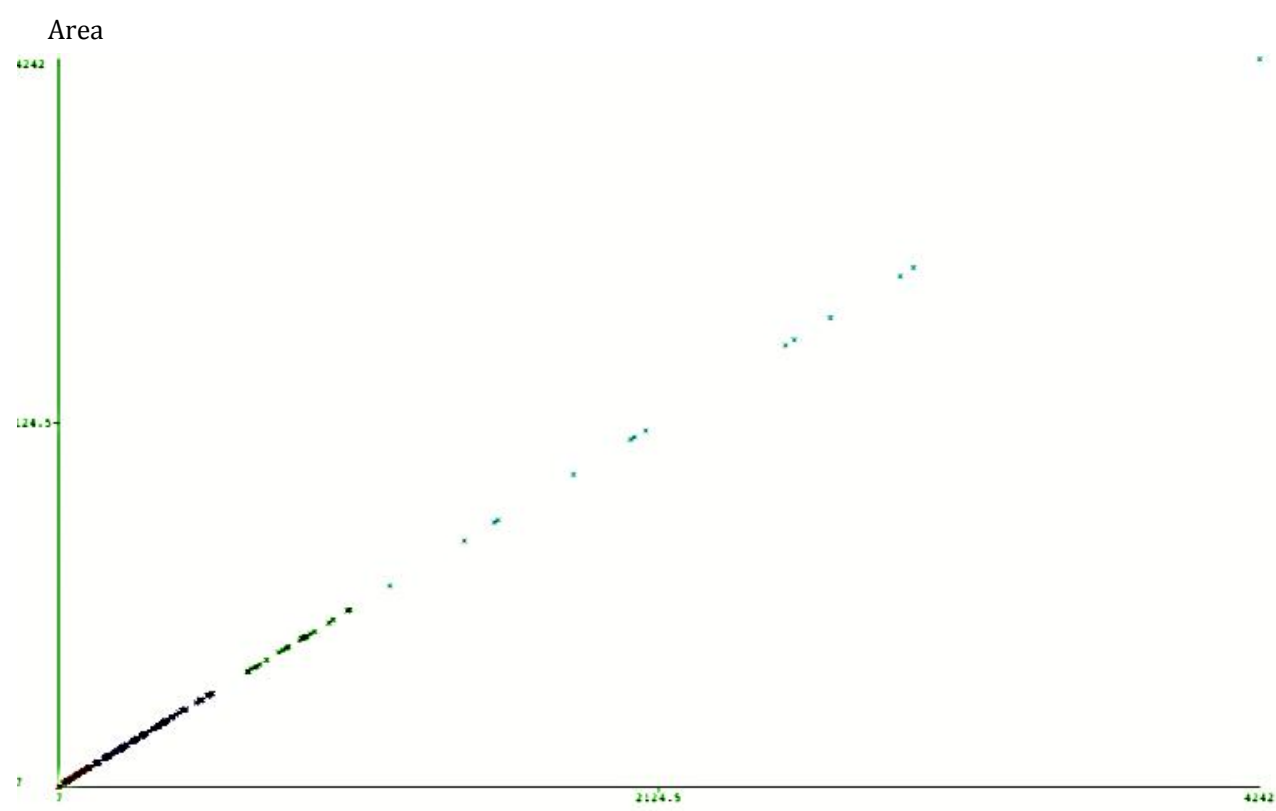

Figure 3-5. Visualization of cluster analysis

The processed data can be exported from Weka to excel. The final output is a table with the data originally introduced but categorized and divided into clusters. Every area analyzed has one identity and belongs to one cluster (cluster $1,2,3$, etc.). This means that the area of every feature (BL, PL, BF) is linked to the cluster number to which it belongs (cluster 1, 2, 3, etc.). Examples of the final results after cluster analysis has been performed can be seen in appendices 11 to 21

\subsection{The use of aggregations and discontinuities to assess resilience in urban landscapes}

In Fig. 3-6 two systems are analysed in terms of the organization of the structure of each. If the same example is used in the analysis of the built environment it will possible to imagine that the constitution of building footprints, for example, is being analysed. A group of buildings from two different places was studied. Results show different structural patterns. The yaxis represents rank size and the x-axis size of element. In the left chart in Fig. 34 the system shows only four aggregations (groups of dots) while in the right chart the system shows six aggregations. The comparison denotes that the structure of the second system is more complex and therefore it has a higher resilience capacity than the first system (C. Allen et al., 2005). 

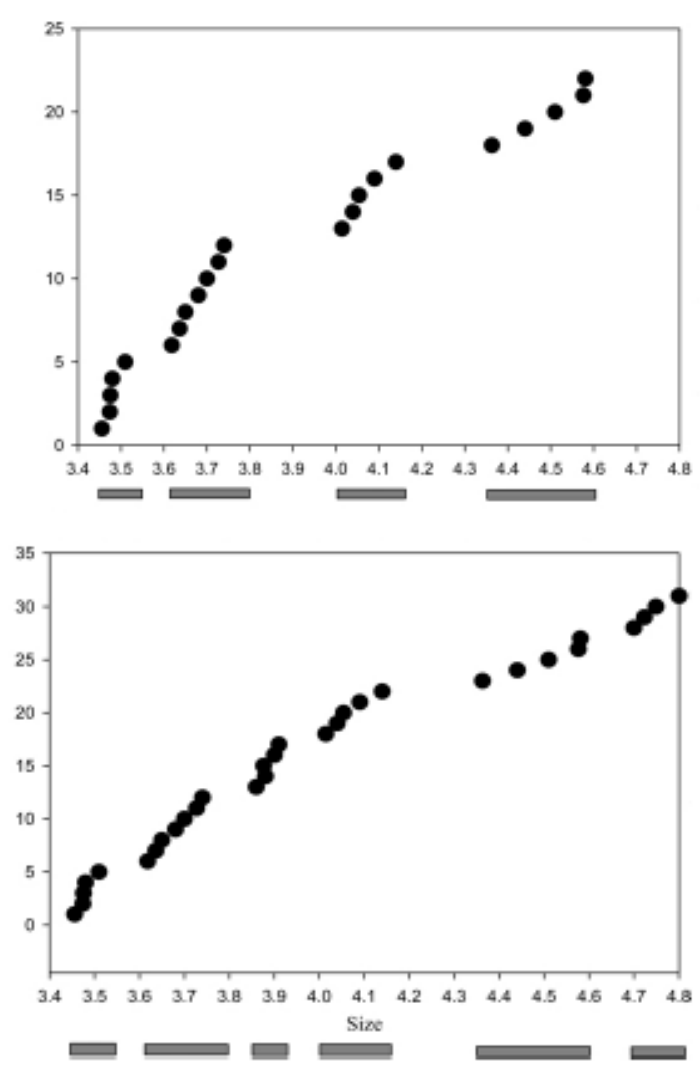

Figure 3-6. Distributions of aggregations and discontinuities in two hypothetical systems

Continuing with the example, the structure of a plot, block, or street at a precise time and scale will be linked with the number of aggregations found at each level. The number of aggregations found in the structure of an urban landscape will be connected with its richness. Diversity and heterogeneity can be calculated by using different indices for their analysis. According to ecological resilience richer systems tend to be more complex and therefore they have more chances to deal with unpredictable change while remaining stable. The final purpose is to characterize the richness, diversity and heterogeneity of a system by analysing the distribution of its information within and across scales.

The analysis of the discontinuities and aggregations in the internal structure of a system can be supplemented with the analysis of the function that elements play within each aggregation and across levels. Functional richness can be measured within and across scales (C. Allen et al., 2005). Within scales, it is possible to evaluate the richness (quantity of functional groups), evenness and 
diversity of each aggregation. The results can be averaged to compare the dynamics between aggregations. Across scales, the functional redundancy defines a parameter for comparing dynamics between scales (C. Allen et al., 2005). Using this framework it might be possible to analyse changes in the resilience of complex systems over a period of time, or between two different systems. For example one that has collapsed can be compared with another system that has overcome similar disturbances. In the built environment this analysis can be very useful, however it demands having information on the function of every element analysed as well as a record of changes in the evolution of those functions.

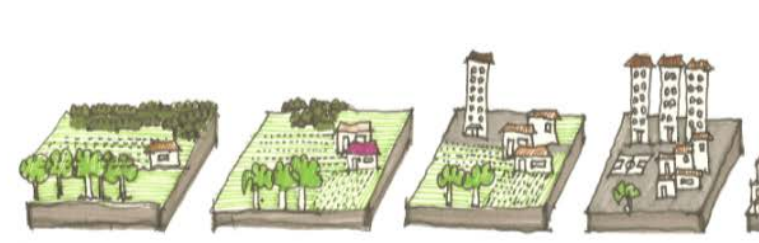

Structure before an event
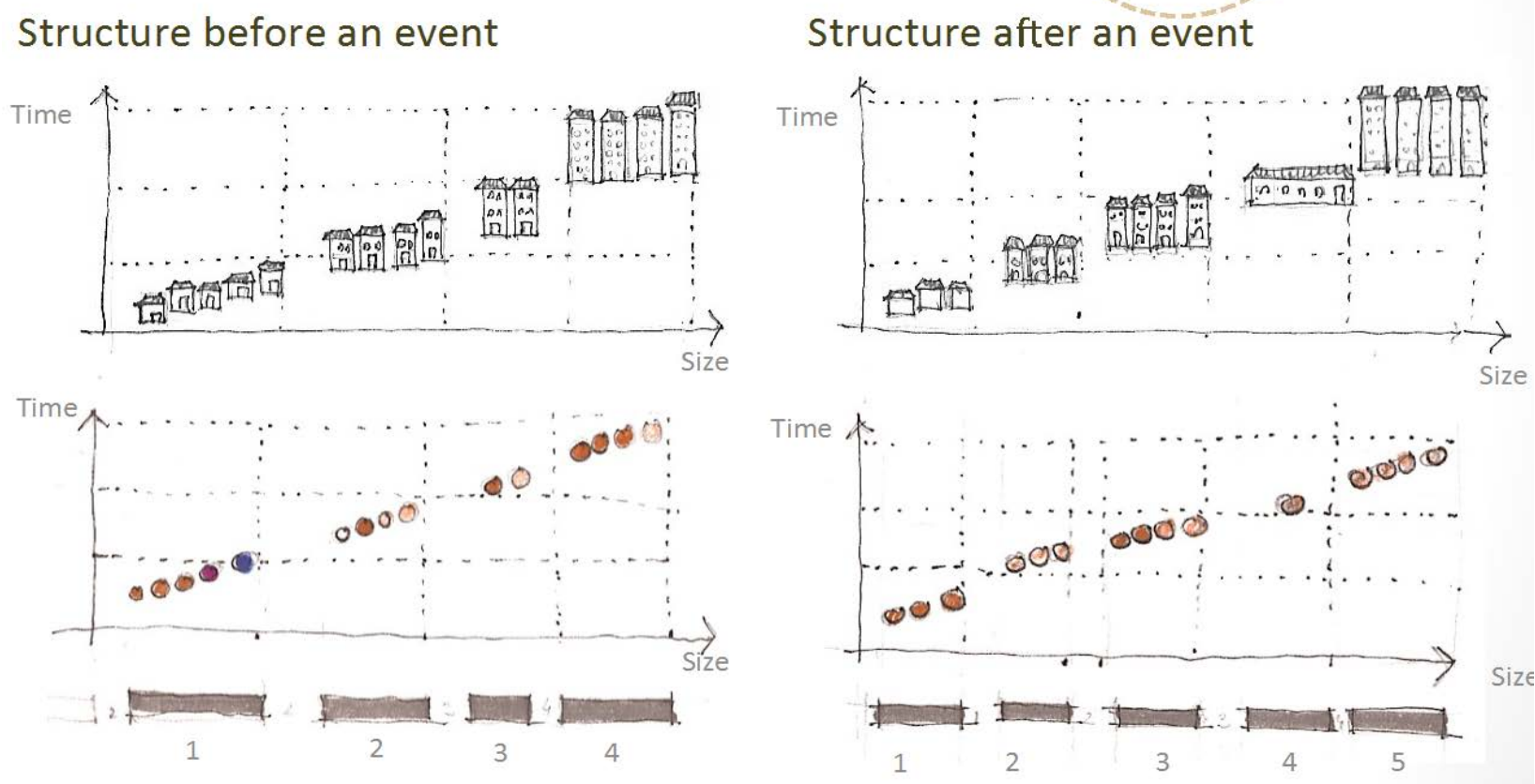

Figure 3-7. Example of the use of discontinuities to assess resilience

A hypothetical example is illustrated (Fig. 3-7) in order to explain the ideas around the use of discontinuities and the measurement of functional richness, diversity, evenness and heterogeneity in the built environment of an urban landscape. In the example, the scale of analysis can be framed at a block 
level. The timeline represents the evolution of one block. The analysis aims to understand the resilience capacity of the urban landscape (block level of resolution) to changes in the density of the built environment. The relative resilience is measured at one particular point, before and after a disturbance, for example the disappearance of houses due to a fire. Charts in the middle of the picture represent the clustering process where buildings are grouped considering how type and density have influenced change in time and rank size. Before the event the urban landscape had four aggregations of building types. After the disturbance, the urban landscape has five groups of building type aggregations. The two charts at the bottom of Fig. 3-5, with coloured spheres show the instance before and after a crisis and exhibit how the distribution of the functional richness has changed between periods. In the stage before the disturbance the structure of the system had four different functions, represented as beige, orange, red, and blue, changing to only two after the event. These results show that the disturbance generated opportunities for new types to be introduced in the urban landscape; therefore it encouraged opportunities for novelties to appear and make the system more complex. However, the functional richness and diversity within and across aggregations has diminished after the fire, implying that the functional heterogeneity of the systems was critically reduced. In conclusion it is possible to infer from the analysis that the urban landscape will show a higher resilience capacity to buffer changes in its density than changes in its functional configuration.

The following three chapters draw together these theoretical discussions by applying the various approaches to selected case study urban areas. 


\section{Assessing resilience and the dynamics of change in the urban landscape of the Auckland CBD}

\subsection{Introduction}

In order to have an overview of the evolutionary changes produced in the built environment of an urban landscape, the development of a timeline is a critical step for characterizing the behaviour of a system at different scales of space and time (see section 3.2.5). This chapter is particularly focused on testing the implementation of a method in which timelines are merged with town-plan analysis, and the observation of the dynamics of change of urban landscapes. Observations will contribute to finding evidence of how to apply the theoretical background of ecological resilience to the analysis of urban landscapes.

The aim of the chapter is to observe if the elements of the urban landscape tend to create a dynamic structure as described in Panarchy. If an urban landscape can be understood as a Panarchy, its elements should be organized in a structure that shows scale breaks in relationship with the different rates of change and size of its elements. Every scale of the Panarchy should exhibit adaptive cycles that can be related with other scales but that are not necessarily dependent on these. In the structure, smaller elements should tend to be grouped at smaller scales and they should tend to change more and faster than bigger elements that should be grouped at bigger scales. If the behaviour described is observed, it can contribute to sustaining the hypothesis that the resilience of urban landscapes can be observed through the analysis of the morphology of the built environment and also that the method proposed indicates a way of applying the ecological resilience theoretical framework to the analysis of the resilience of urban landscapes.

The study can provide important information about the understanding of the historical sequence in complex adaptive systems like socio-ecological landscapes (see section 2.2.1). Furthermore, at the same time the aim to see if it is possible to highlight events and disturbances in order to investigate patterns of changes, discriminate periods, and classify internal crisis and events. 
The first part of the chapter is a qualitative analysis that uses timelines as a method. The timeline is a reference for comparing the evolution of the built environment with external disturbances that have been originated in the context. The second part of the chapter is a quantitative assessment of the timelines that describes phases in the evolution of the built environment by quantifying change at different periods. The outcome is to try to observe if the built environment of the urban landscape can be analysed as an urban Panarchy in space and time. The final aim of the study is to find more information about the dynamics of change of urban landscapes and to test the potential of the resilience theory to be applicable to the analysis of urban landscapes.

\subsection{About the case study}

With the aim of making a bridge between the morphological and ecological viewpoints the present study developed a methodology and methods for making a historical qualitative and quantitative analysis of the urban landscape of the east side of Auckland CBD. In this chapter the case study is used only to test the theoretical background developed. The subject of analysis can be any city in the world, and without it exhibiting any particularity. The fact that Auckland was used as a case study represents an excuse to create open debate in New Zealand about urban resilience. The east section of Auckland is analysed because the place contains a palimpsest of different historical periods (from the origins of the city until today), is still part of the CBD of the city, contains a variety of elements of the urban landscape, and the scale and amount of information presented in the urban landscape of the sector is manageable for analysis using the approach developed.

\subsection{Qualitative analysis using timelines}

The theoretical foundations for making a timeline (see section 3.2.5) are based in studies done in morphology (Slater, 1990; Whitehand, 2007), in ecological resilience (Resilience Alliance, 2007a), and that are also sustained in a philosophical approach (De Landa, 2006). The methodology mixes a multiple scale approach (see section 3.2.6) and the analysis of cyclical change derived from ecological resilience with the measurement of transformations in built 
environments from morphogenetics. The method consists of overlapping general historical periods from the available sources and inferred breakpoints or shifts of state.

In morphogenetics, the investigations have shown that the morphogenesis of the city is strongly linked with economic and social forces that become drivers of the urban landscape, and that these define phases of change. In this approach phases are inferred from the external and contextual forces that transform the urban landscape (Conzen, 1969; Slater, 1990). The first section of the qualitative analysis was done following similar criteria. Nevertheless, in ecological resilience phases in the process of adaption of a system are defined by analysing disturbances and shifts of state at different scales (Folke et al., 2004) and domains in time and space (Resilience Alliance, 2007a; Redman \& Kinzig, 2003). The history of a system is told as an analysis of the performance and behaviour of particular issues in the face of change. In consequence, phases of change in the situation of a system are deduced as a synthesis of the interplay between internal and external forces. The definition of domains, drivers of change, and scales becomes key information because the analysis of their behaviours is linked with future changes in the system (see section 3.2.1). The second part of the qualitative observations is focused on this approach having the urban landscape of Auckland as the focal system and different domains as its context.

The method consists of collecting and grouping different historical narratives from discrete viewpoints. The idea is to describe "grosso modo" the most relevant event and processes that occurred in the context of the urban system and also in the morphology of the urban landscape. In the timeline, events are highlights in a timeline that by themselves establish breaking points (black dots in fig 4-2). Processes are a set of actions and tendencies that develop over longer periods of time. Following the ecological resilience theoretical framework, the main periods in economics, society and politics are included as a reference. Events and processes were included considering the possible importance of each for the general history of the system or for its impact on the transformation of the urban landscape at different scales. The last condition is very subjective because some processes and events are easier to track and to 
relate than others. The intention was not to link all events and processes but to build a 'cloud' with them, where new connections can be discovered.

The aim is to produce a panorama of the different phases in the evolution of the identity of urban landscapes as resilient assemblages constituted by multiple scales of space and time (see section 2.3) that work in a complex and non-linear way. This analysis can provide evidence that the built environment of urban landscapes produces patterns and interrelationship that are non-linear.

\subsubsection{Timeline at the scale of New Zealand}

It is difficult to find consensus in ways of dividing the history of New Zealand because every author implements a particular approach and criteria with which to characterize historical periods, and sometime these are not very well defined. However, it is possible to find similarities in the quantity and number of periods found, and consequently these have formed the criteria used in this section. The literature reviewed (Hodgson, 1990; King, 2003; McLauchlan, 2005; Oliver, 1981) that deals with the history of New Zealand tends to settle, generally, on between four and six named periods.

The first period, before European arrival (until 1840), is usually dedicated to analysis of the Polynesian culture and the first settlement of New Zealand before the European arrival (McLauchlan, 2005). The second period (18411900) deals with land wars and the beginning of a colonial economy (McLauchlan, 2005). The birth of a colonial economy (Hodgson, 1990) characterizes the end of this period of growth and violence (Oliver, 1981). The third period (1901-1952) usually deals with the "transition" (Oliver, 1981) from the colonial to the modern New Zealand. It includes the world wars, depressions, and political changes until the end of the Second World War. The third period (1953-2011) is focused on the development and globalization of New Zealand from the Second World War up to the present (Oliver, 1981). This period can be subdivided in two phases: before and after the 1970s. Before the 1970s, the country was described as experiencing a period of consolidation and also prosperity (McLauchlan, 2005) after the difficult times of the wars and depression. After the 1970s, the country is dealing with its insertion into a globalized world. 


\subsubsection{Timeline at the scale of Auckland}

The bibliography that deals with the history of Auckland is neither diverse nor extensive. Bush (Bush \& Council., 1971; McLauchlan, 2008) divides the history of Auckland into five periods:

1) The government of Auckland before 1871,

2) Establishment of the pattern of council activity (1871-1918).

3) The modernizing city (1919-1945).

4) Polis into metropolis (1945-1971).

5) The first century surveyed (1971).

In 1998, Bush in a condensed summary included two new periods:

6) Progressing towards abolition (1971-1989).

7) Write large: the 'new' City Council from (1990-1998).

However the criteria that define each period are not clearly stated.

Nonetheless, the socio-economic definition of historical phases can differ from the characterization of the different stages in the evolution of Auckland from an urban approach. As explained in section 2.3, another possible way of defining the phases of evolution in an urban landscape system could be achieved by defining changes in the identity of a place. From this point of view, the definition of the identity of an urban landscape can be used as a metaphor through which to comprehend critical changes and to delimitate phases in the adaptive cycle of a socio-ecological system. The following characterization was done based in a report (Section, 1969) and adapted using the viewpoints discussed in chapters 2 and 3. Periods were renamed in terms of adaptive cycle phases.

\section{Exploitation (1840-1850)}

The first settlement of Auckland was shaped by social and ecological factors as well as top-down and bottom-up decisional systems that created a hybrid image of the city. The general character of Auckland in this first period was "scruffy" (Section, 1969, p. 15). Its inhabitants were focused on surviving and subsisting. Consequently, the buildings were precarious, being made of timber and surrounded by ferns. Both the economy and survival depended on the food that the Maori supplied by canoe. Shortland Crescent was the most important 
street. It linked the port with Britomart Point, the hill where the few government and institutional buildings were settled (now Emily Place). Here there was the main church, St Pauls, which was the most emblematic building of the landscape and also Fort Britomart, Government House and the Albert Barracks. Princes Street was the place where all the elite were housed. Official Bay received its name because it was the place where the elite of the officers lived, an area well known by its exclusivity. Mechanics Bay and Freemans Bay were the places where the working class lived. The infrastructure of the settlement was insignificant. The movements in the settlement were done by walking and by water transport.

\section{Conservation (1850-1870)}

The population, urban and agricultural land expansions that started around 1850 helped to consolidate the city. The character of Auckland was a mix between a "garrison town" (McLauchlan, 2008, p. 31) and a "watering place" (Section, 1969, p. 23), where soldiers could rest after a military campaign against the Maori. The permanent community remained compact around Queen Street valley. The identity of the urban form of Auckland resided in the interrelationship between the harbour, and the land and city form. The extension of Commercial Bay and the prolongation of Queen Street out into the sea generated a commercial pier that was a key social and commercial point, the icon of the city. Buildings were positioned in ridge locations around the Queen Street valley, highlighting the shape of the landscape. In this period Queen Street became the most important street establishing a change of direction in the growth of the urban landscape. Fires in Shortland Crescent and available land in Queen Street made the shift possible. In this case, fires represent socio-ecological threats that combined and exposed the cultural and ecological weakness of the system.

When the capital of New Zealand was moved to Wellington, the economy of Auckland that was based on tertiary services faced big challenges. One of these challenges for Auckland was the development of its agricultural activities in order to encourage the necessary diversity to overcome the disturbance caused by the breaking of the dependence on the government and military institutions. 


\section{Collapse (1870-1915)}

The depression of Auckland was faced by developing economic activities through the exploitation of farm, forest, coalmines, goldfields and gum fields along with the consolidation of population growth and the intense trade between Sydney, Auckland and San Francisco.

Innovations in the transportation system produced a rapid extension of the urban areas incorporating new neighbourhoods in the urban fringe. This factor strengthened the centrality and importance of the Queen Street valley, with it becoming the node of transportation, commercial and industrial activities. The demolition of Britomart Point (early 1880s) and the Albert Barracks were breaking points in the urban landscape form. The development of the industrial belt along with the railway infrastructure cut the link between city and sea that characterized the settlement in the past. At the street scale these changes produced the opportunity to develop Custom Street East, which created a new façade for the city, leaving Shortland Street (formerly Shortland Crescent) as a second façade. In an adaptive cycle it could be possible to say that this period is the transition from the collapse of a conservation period to the reorganization of a new cycle.

\section{Reorganization (1915-1969)}

This is a period of a rapid peripheral growth. Industrial settlements and suburban developments impact with the loss of a traditional centrality in the developing of a metropolitan Auckland. Highways catalysed the utilization of motor transport, increasing the flow of goods that were carried for storage and processing around the harbour. By expanding wharves, harbour reclamation reached 400 ha in 1966. The industrial development produced a population growth. Suburban areas absorbed this population growth while the urban centre experienced an inverse trend. The land use in the central area exhibited some changes, for example in the occupation of old industrial places by warehousing activities behind Queen Street, mainly in 1930. The impact of the motorcar in the urban landscape can be observed in the proliferation of car servicing shops, garages (Albert Street, Shortland Street, Chancery Street and the Market Square), and also in the quality of the streets. The emergence of the High Street-Lorne 
Street system illustrates a tendency for having smaller pedestrian streets that appeared after World War II. The other important impact in the urban landscape was the extension of the university in Princes Street.

\section{Conservation (1970-2012)}

Broad economic deregulation, intense change, the processes of entering into a global economy, population growth and pressures on transport, housing and infrastructure are characteristics that have had an impact on the urban form of Auckland in the last 40 years.

\subsubsection{Synthesis of timelines: morphological changes in the urban landscape of the Auckland CBD}

This historical approach is aiming to be a first step in trying to build a timeline of the key events and processes that have characterized the evolution of the context of Auckland. The analysis is based on the April 2010 report prepared by a team for the Auckland Regional Council (Council \& team, 2010). The report describes the evolution of the urban form of the metropolis from colonial times up to the present day. The review is chronological and makes a parallel between the key drivers of the context and their influence on the configuration of the urban form of the region (see Appendix 1).

In ecological resilience the dynamics of change in ecosystems are controlled and driven by a few sets of key structuring processes and variables that attract and define the path for all the rest. In this framework, structuring processes are defined that generate and are impacted by the structures they produced (Holling, 1992). This timeline (Fig. 4-2) was constructed for Auckland following the same criteria. Structuring processes are the drivers of change for each period, and structuring variables are concrete outputs generated by structuring processes that will have an impact on the morphology of the urban landscape.

The objective of this timeline is to map the group of processes and events of the entire socio-ecological system that contains the urban landscape of Auckland, at discrete scales (New Zealand, Auckland and Auckland CBD scale) and through different domains, (economic, politic, social, technological) in order 
to try to establish connections between these events and processes and their impacts on the morphology of the urban landscape. Processes are represented by horizontal lines that cover the time they were around and are based in the structuring processes. Events are dots placed at the moment when they emerged and are based on the structuring variables.

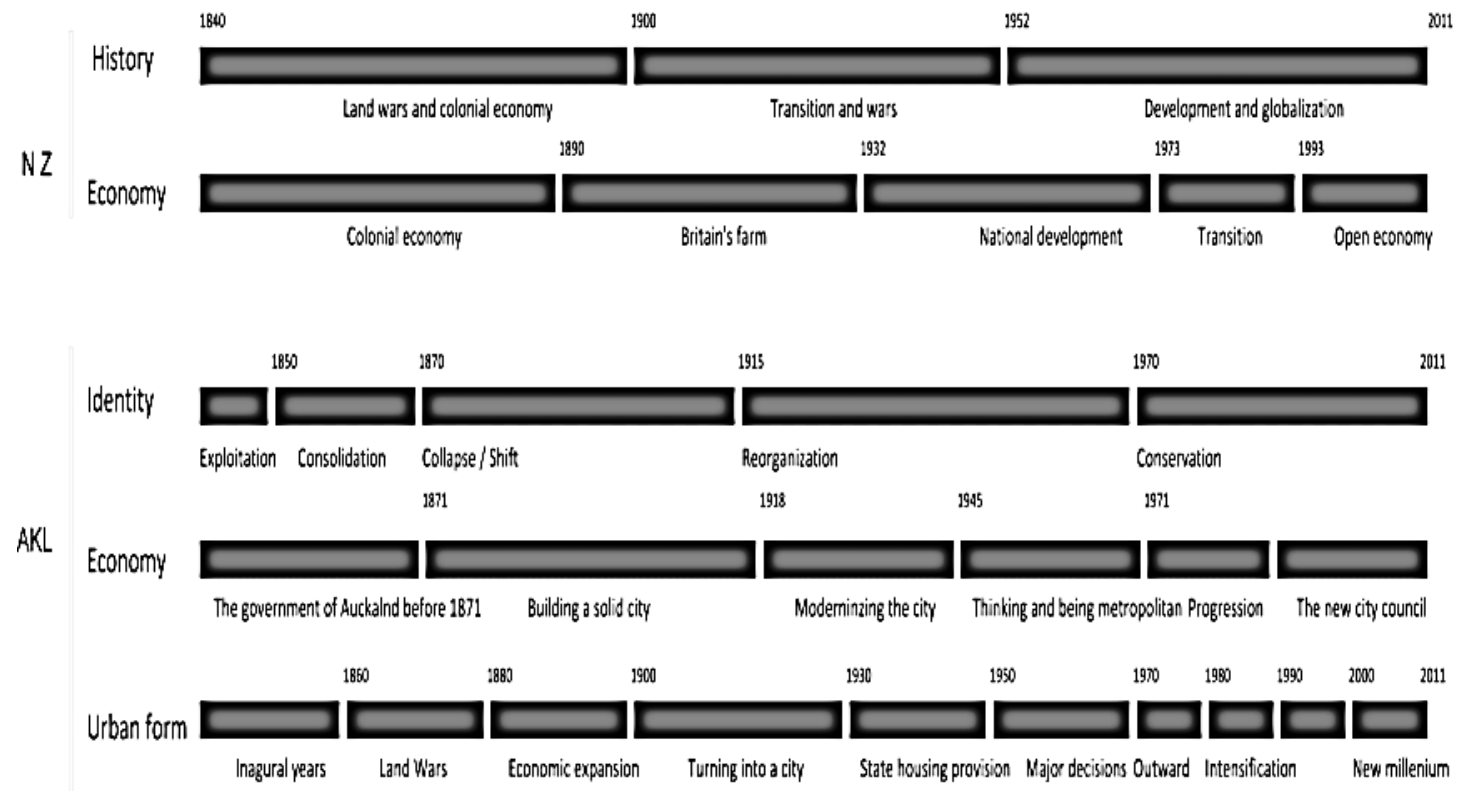

Figure 4-1. Multiple historical phases at different scales 


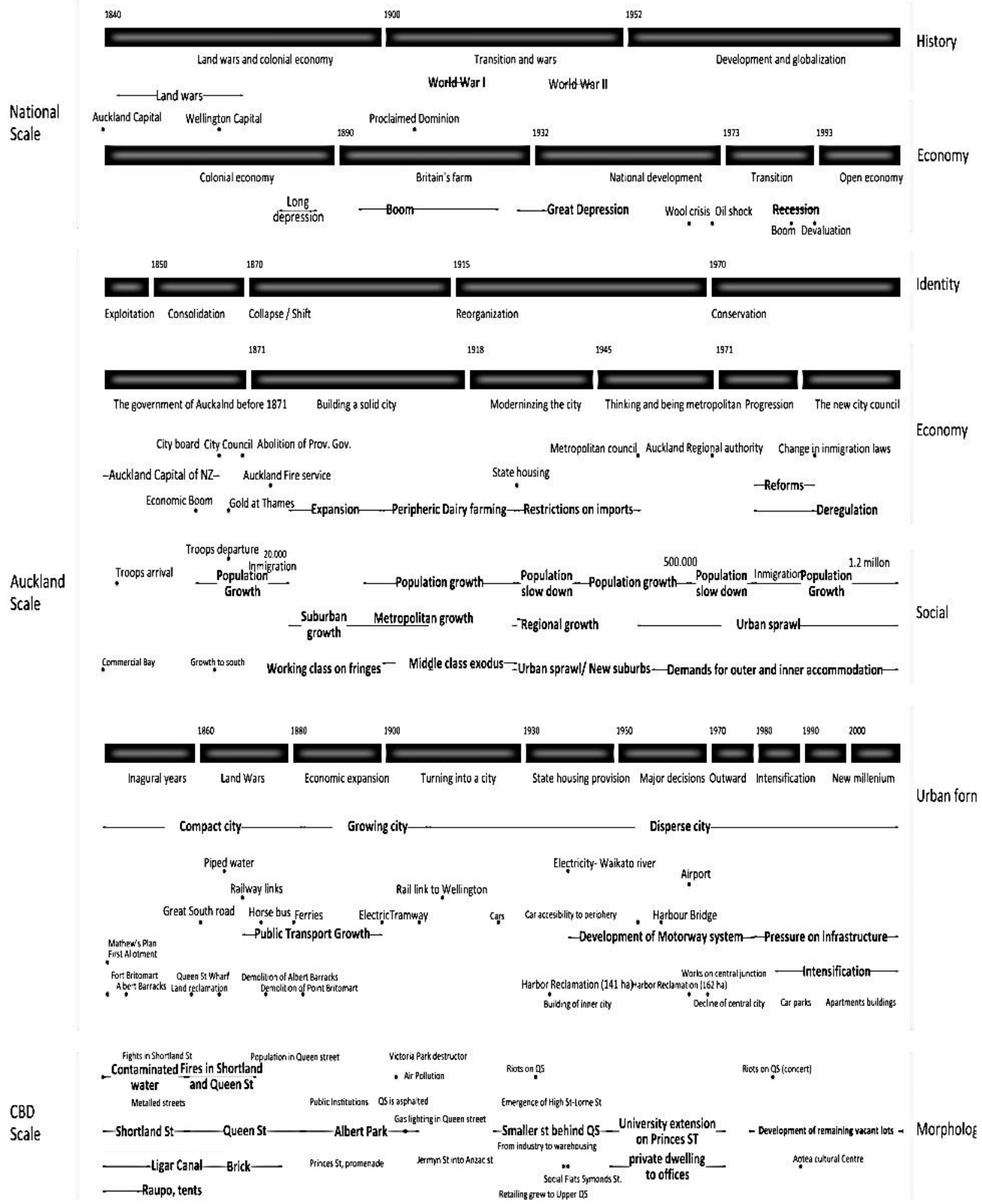

Figure 4-2. Timeline 


\subsubsection{Observations about the qualitative approach to timelines}

The timeline gives the possibility of linking processes and events across scales, producing the emergence of structures and paths that were not clear before. Historical periods at bigger scales tend to be longer and fewer than periods at smaller scales that are more numerous and therefore more frequent. Historical periods that synthesize more than one domain are broader but help to group and frame others that are more specific and related with one particular domain. Periods tend to be shorter at all scales from the 1950s, probably as a symptom of the frequent crises that have characterized the unstable identity of the contemporary urban landscape of Auckland (Fig. 4-1, Fig. 4-2).

The time line reveals that the sequence of events and processes at bigger scales does not always produce an impact at lower scales. Therefore, not all the transformations at CBD scale are produced by top down decisions neither can they be totally explained as a linear consequence of processes and events that occurred at bigger scales. The possibility of inferring connections between events and processes across scales does not represent a linear cause and effect chain of event and processes, but reveals the conformation of structures that show that the clustering of events and processes is more intense in some parts of the timeline than others. This fact can be evidence for the aggregations of patterns that occur in complex adaptive systems, and that emerge when processes are linked within and across scales (Fig. 4-2).

\subsection{Quantitative analysis using timelines}

The present study is focused on the quantity of change that the built environment of the urban landscape of the east side of Auckland CBD has produced, by analysing its main structural elements. The present analysis could help to support or contest the assumption that the observation of the dynamics of change within and across scales in the elements of an urban landscape can be compared with the dynamics of change described in Panarchy. The presence of such a Panarchy would contribute to providing information about the resilience attributes of an urban system. Basically the urban Panarchy will expose two points: that urban systems are complex and that this complexity can be shown in 
their organization and dynamics. In turn, this can help to illustrate how adaptive processes actually work in space and time.

The quantitative analysis measures the rhythm of change in key elements of the built environment of the east side of Auckland CBD, these being building footprints (BF), plots (PL), blocks (BL) and streets (ST). The variables analysed were considered, theoretically, as key elements (species) and drivers of the structure of urban landscapes (see section 2.5.2).

A complex approach to measuring the evolution of the urban landscape in question relies on measuring the evolution of the system in space and time (computed areas for nine periods), at four different levels (BF, PL, BL, ST), using three variables (sum, number, mean) for each level of the urban landscape. By using this approach it is possible to tackle one phenomenon from multiple dimensions.

The investigation was approached in three stages. First was making a general assessment of the overall evolution of the urban landscape in order to have an overview of the performance of the system. The second stage was to analyse in depth the evolution of each variable and the relationships of the variables within and across scales. The analysis was done by assessing change in the variables BF, PL, BL and ST in terms of their quantities and rate of change. The third part of the study was the diagnosis of both quantities and rates of change. The objective was to synthesize the magnitudes analysed in order to define possible phases in the evolution of the built environment.

The analysis of the evolution of the urban landscape of the east part of the Auckland CBD was carried out using historical maps that contain at least plots, blocks and streets and by redrawing the maps in QGIS (see section 1.12).

The quantity of change in space was assessed by measuring the difference in change as a percentage of change. By using the percentage of change it was possible to measure and compare the quantity of change in simple terms, such as whether change is big or small. The percentage of change represents the difference between the value of a variable in one period and the new value of that variable in the next period, multiplied by 100. In order to measure the percentage of change the absolute difference, that is always positive, was used. The database was built by taken the area $\left(\mathrm{m}^{2}\right)$ of BF, PL and BL, for the historical 
periods previously chosen. In the case of ST, the length in linear m was used as a reference. Three variables were implemented in the measurement of BF, BL, PL, ST: the sum, the number of features, and the mean value. The sum was relevant to understanding the total amount of change in space, the number of features was more relevant for variables that do not change in their areas but that do in their composition, and finally the mean was implemented because it represents the relationship between the sum and the number of features, thus giving information about the proportion of the growth in a space. Consequently, the amount of change was measured by assessing the sum, number and mean difference for BF, PL, BL and ST between the chosen start and end dates.

The quantity of change in time was measured by using the average rate of change of the sum, number and mean values of BF, PL, BL and ST for the selected periods. The average rate of change was calculated by using the percentage of change of the sums, number and mean values at each level divided by the number of years in each period (see appendix 4).

\subsubsection{General Observations about change in BF, PL, BL and ST}

When observing the evolution of the elements of the urban landscape (Fig. 4-3) there are two clear observations: firstly, every element has its own domain and range in the chart; secondly none of the elements has evolved in the same way. They are neither equal to one another nor linear in their developments. There is no intersection between the ranges that every element holds; therefore, it is possible to affirm that every element has its own domain. This clear distinction of ranges, where blocks represent the biggest quantities, plots the second largest and building footprints the smallest, emphasizes the fact that each one of them works at a different level in the same urban landscape. This is clear evidence for the complexity of urban landscapes and also exposes part of the challenge of analysing them. 


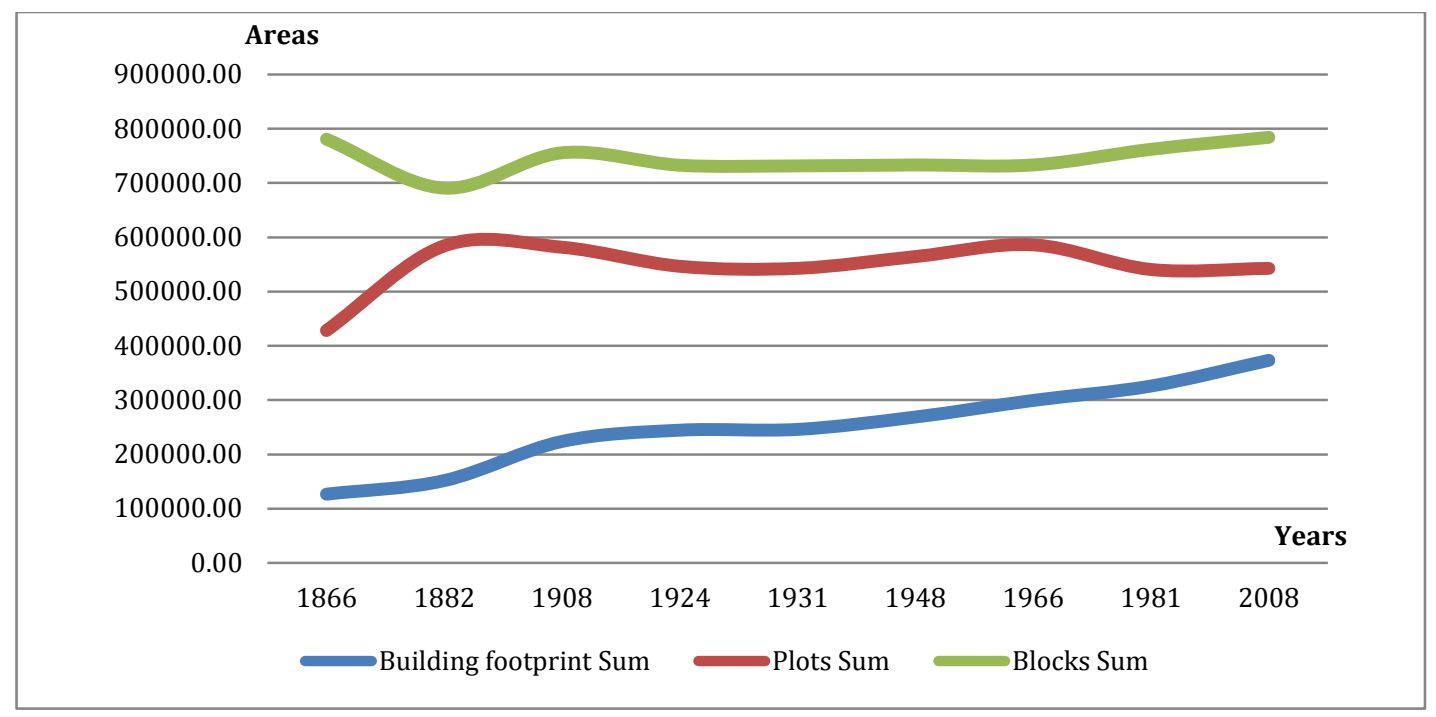

Figure 4-3. Evolution of total areas (sum) of BF, PL, BL

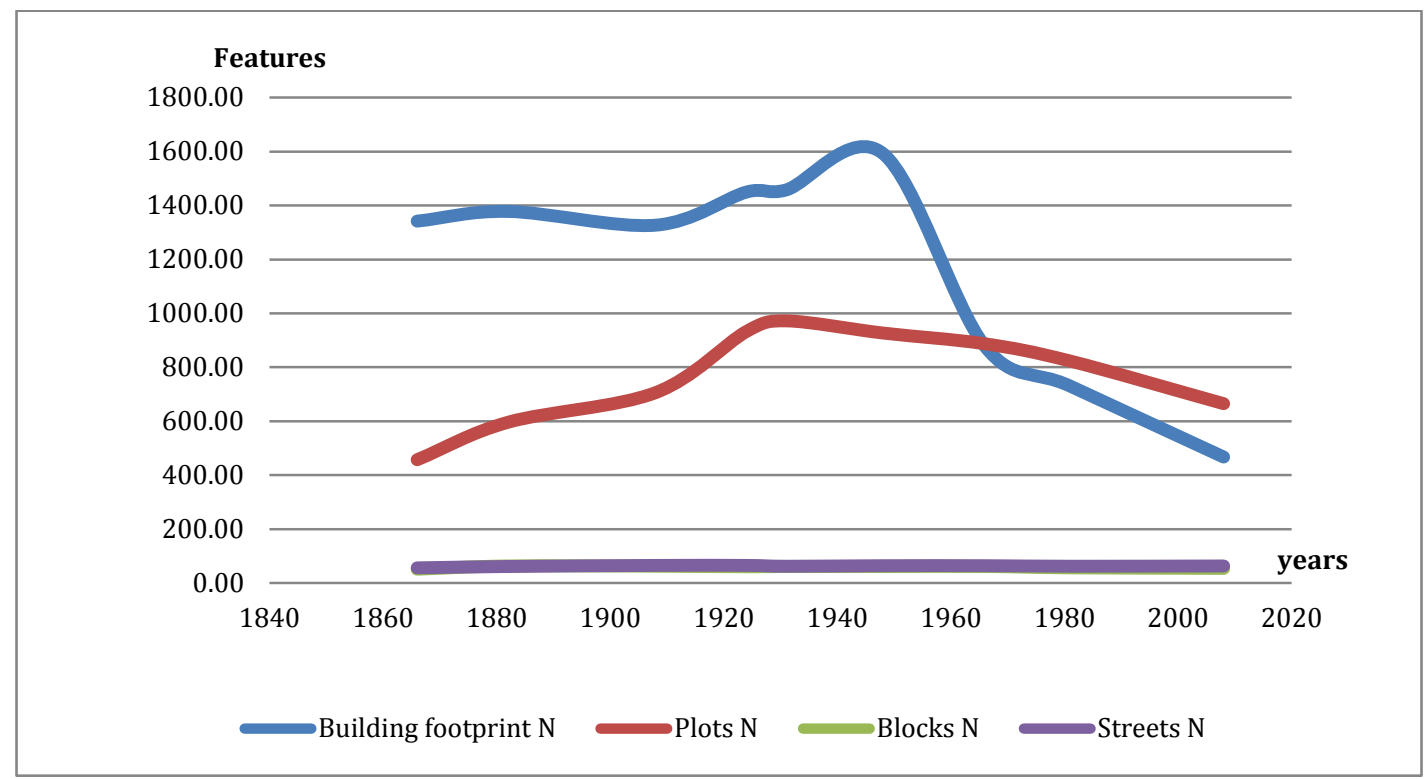

Figure 4-4. Evolution of number of features per BF, PL, BL, ST

Through the observation of the evolution of the sum areas, it is possible to recognize that building footprint is the only variable to exhibit a constant increment in its sum and it is also the most changeable variable. One reason for this is that the growth of plots and blocks outside the boundaries of the case study are not considered. The only exception in the evolution of the total areas in the rest of the levels can be observed in plots during the first interval 1866-1882. The cause of this alteration was probably the demolition of the Albert Barracks. Because green open public areas are not considered as plots in this research, 
changes from private to public areas are susceptible to changing the plots sum value. Streets were not considered in the first chart (Fig. 4.4) because they are quantified in linear metres not by area.

When looking at the evolution through the quantification of the number of elements at each level, BF and PL exhibit an evolution with different phases while BL and ST seem to be very constant. BF has a peak in the 1940s, which divides the curve into two parts, one of incremental change (1860-1940) and the other one of decline (1940-2008). At the same time, the incremental part could be divided in two phases: small change from 1866 to 1908 and increasing change from 1908 to 1948. The second phase has an important decrease until 1966 and a less critical one until 2008. Plots show a less pronounced curve with a higher point in the 1930s. BF and PL curves intersect in 1966. From that moment they follow similar slopes until 2008. Fig.4-4 shows the number of small elements, like BF and PL, changes by bigger amounts and more frequently than the number of bigger elements, like ST and BL. The measurement of all these variables in their absolute quantities helps to show what their changes mean to the whole system; however it does not clearly expose how much they are changing within their own scales, especially in the case of BL and ST. For this reason the assessment of the quantity of change was performed by analyzing the percentage of change between start and end dates.

\subsubsection{Quantity of change in space: percentage of change of BF, PL, BL, and ST}

It can be observed that there are two meaningful points where all variables intersect, these being 1931 and 1981 (Fig. 4-6). Taking these points as references, they define three moments, however if taking into account the rhythm of change of each variable it is possible to synthetize the whole chart into two parts, before and after 1924. The first phase from 1866 to 1924 is characterized by big changes in BF, PL, ST and a small change in blocks. BF is the variable that changed the most, followed by PL. In this phase it is possible to observe the difference in the frequency of change between variables. While ST and BL have only one decreasing phase, BF and PL have two phases, with increasing and decreasing values. In the second phase PL, BL, ST are changing in 
a range of around $10 \%$ while $\mathrm{BF}$ is a little above them. A pattern is recognized after 1981, where all variables with the exception of BF follow similar trends.

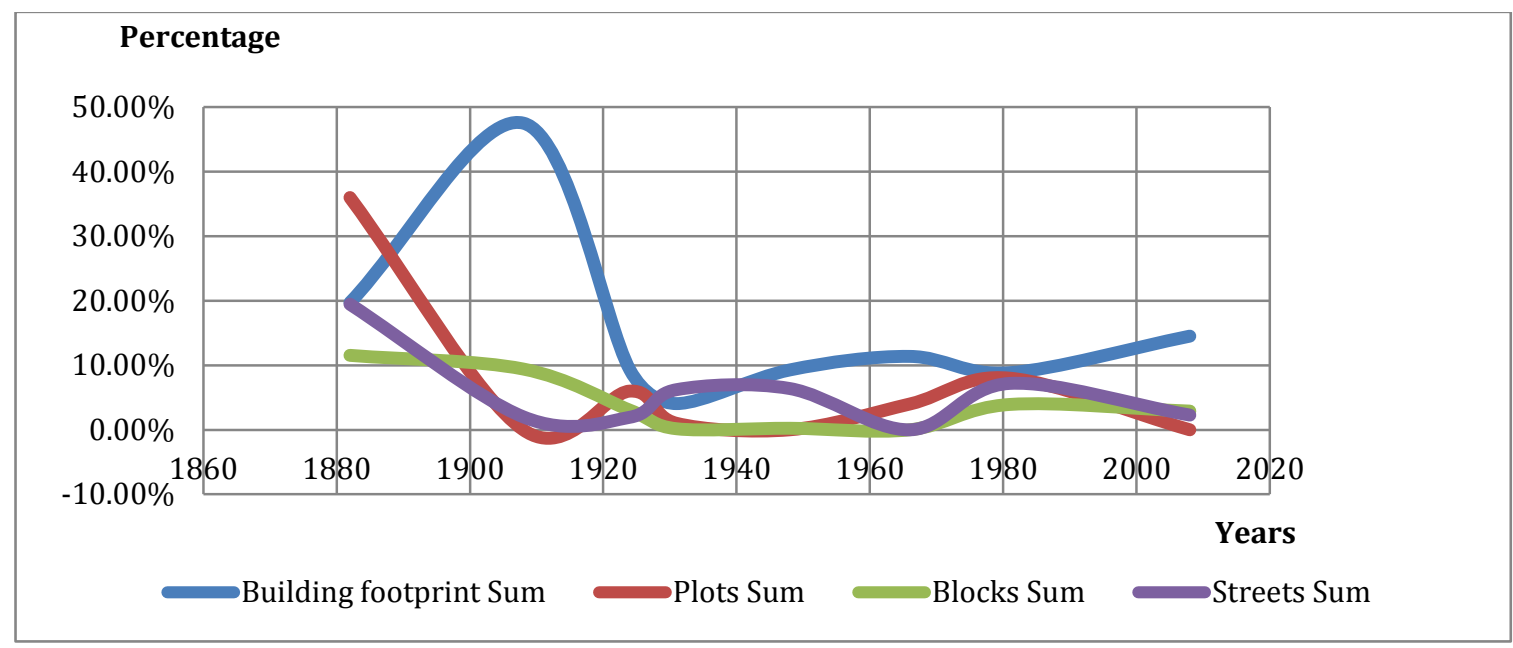

Figure 4-5. Percentage of change in the sum of BF, PL, BL, ST

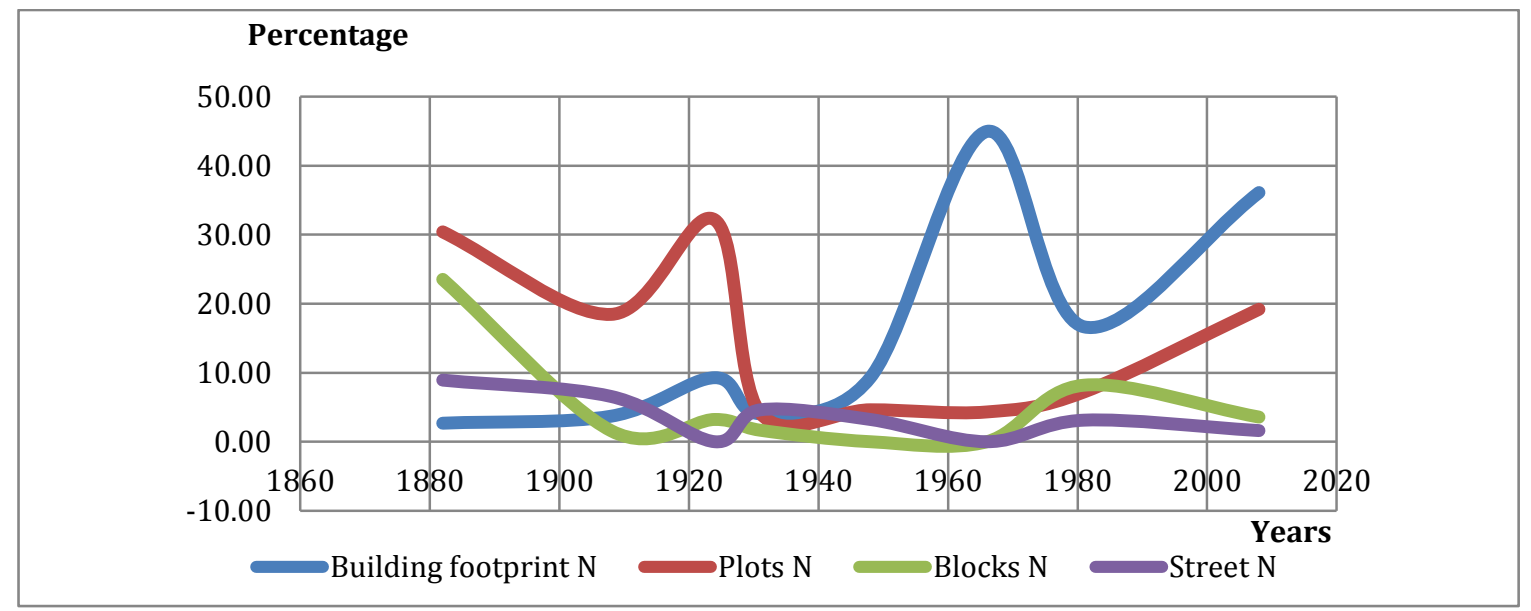

Figure 4-6. Percentage of change in the number of BF, PL, BL, ST 


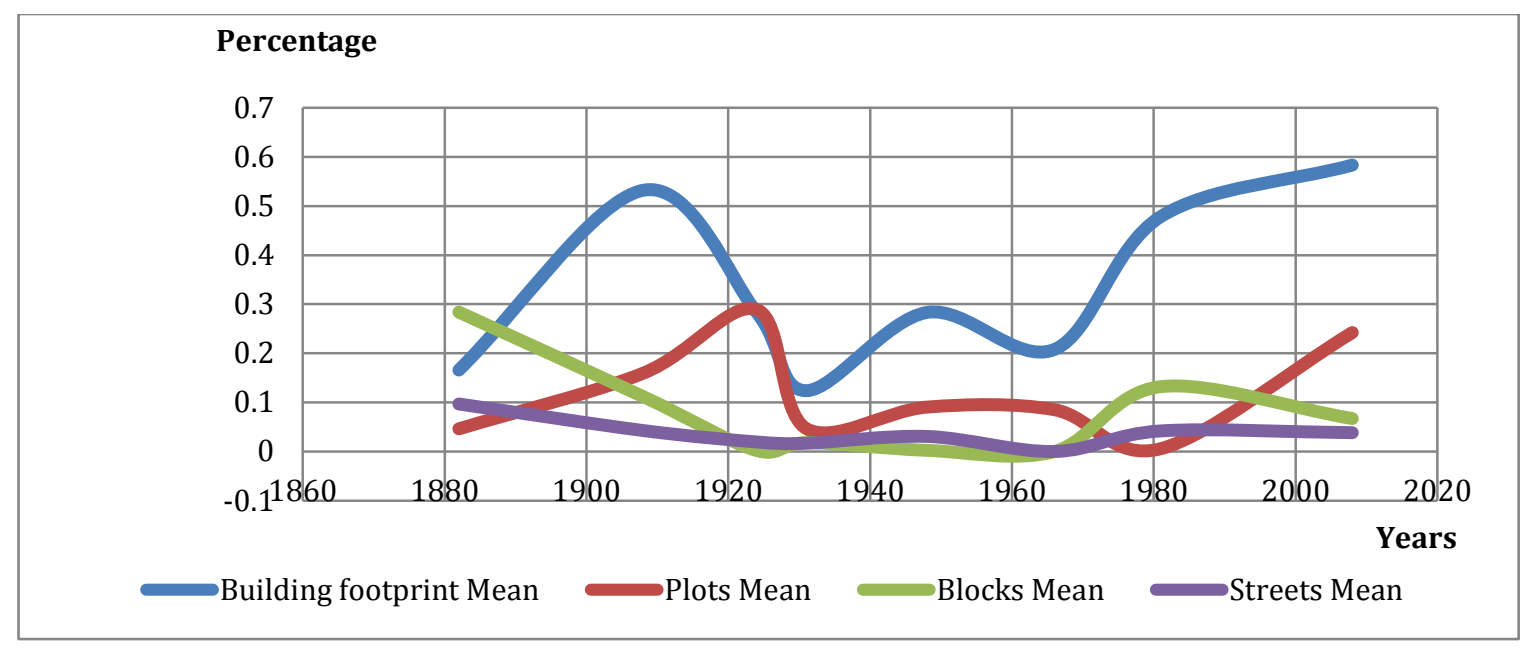

Figure 4-7. Percentage of change in the mean of BF, PL, BL, ST

\section{Categorization of the quantity of change in space}

In order to try to define discrete phases in the evolution of the urban landscape, the results obtained in the measurement of each period were averaged within and across levels, i.e. in each category (BF, PL, BL and ST) and per period across categories. This average becomes a self-reference to define the magnitude of the change at every period. Following the criteria discussed in chapter 3 with which to approach history, breakpoints were examined. Values across categories that are above the average enter in the category of big change while values under the average join the category of small change. The grey boxes in table 4-1 highlight times with values above average. Because absolute values were used only positive numbers are obtained, so this can represent both increasing and decreasing amounts of change. By using this method it is possible to picture how much change is too much or too little.

Table 4-1. Average rate of change in BF, PL, BL, ST (space)

\begin{tabular}{|l|l|l|l|l|l|l|l|l|l|l|}
\hline & & $1860-$ & $1882-$ & $1908-$ & $1924-$ & $1931-$ & $1948-$ & $1966-$ & $1981-$ & \\
\hline \multirow{5}{*}{ BL } & 1882 & 1908 & 1924 & 1931 & 1948 & 1966 & 1981 & 2008 & Average \\
\cline { 2 - 11 } & Mean & 16.59 & 53.20 & 28.32 & 12.39 & 28.32 & 20.79 & 47.85 & 58.33 & 33.22 \\
\cline { 2 - 11 } & Sum & 19.71 & 47.53 & 9.28 & 4.06 & 9.28 & 11.38 & 8.85 & 14.52 & 15.58 \\
\cline { 2 - 11 } & N & 2.68 & 3.70 & 9.28 & 4.06 & 9.28 & 44.98 & 16.65 & 36.11 & 15.84 \\
\hline & Average & 12.99 & 34.81 & 15.63 & 6.84 & 15.63 & 25.72 & 24.45 & 36.32 & 21.55 \\
\hline \multirow{3}{*}{ PL } & Mean & 4.61 & 15.95 & 28.90 & 4.67 & 9.04 & 8.51 & 0.58 & 24.23 & 12.06 \\
\cline { 2 - 11 } & Sum & 36.00 & 0.00 & 6.00 & 1.00 & 0.00 & 4.00 & 8.00 & 0.00 & 6.88 \\
\cline { 2 - 11 } & N & 30.42 & 18.46 & 32.15 & 4.18 & 4.63 & 4.31 & 7.22 & 19.20 & 15.07 \\
\hline
\end{tabular}




\begin{tabular}{|l|l|l|l|l|l|l|l|l|l|l|}
\hline & Average & 23.68 & 11.47 & 22.35 & 3.28 & 4.56 & 5.61 & 5.26 & 14.47 & 11.34 \\
\hline \multirow{4}{*}{ BL } & Mean & 28.39 & 11.18 & 0.20 & 1.80 & 0.24 & 0.00 & 13.21 & 6.71 & 7.72 \\
\cline { 2 - 11 } & Sum & 11.54 & 9.42 & 3.03 & 0.16 & 0.24 & 0.00 & 3.93 & 2.90 & 3.90 \\
\cline { 2 - 11 } & $\mathrm{N}$ & 23.53 & 1.59 & 3.23 & 1.67 & 0.00 & 0.00 & 8.20 & 3.57 & 5.22 \\
\cline { 2 - 11 } & Average & 21.15 & 7.40 & 2.15 & 1.21 & 0.16 & 0.00 & 8.44 & 4.39 & 5.61 \\
\hline \multirow{5}{*}{ ST } & Mean & 9.67 & 4.27 & 1.94 & 1.68 & 3.07 & 0.00 & 4.20 & 3.83 & 3.58 \\
\cline { 2 - 11 } & Sum & 19.47 & 2.01 & 1.94 & 6.22 & 6.39 & 0.00 & 7.19 & 2.28 & 5.69 \\
\cline { 2 - 11 } & N & 8.93 & 6.56 & 0.00 & 4.62 & 3.23 & 0.00 & 3.13 & 1.61 & 3.51 \\
\cline { 2 - 10 } & Average & 12.69 & 4.28 & 1.30 & 4.17 & 4.23 & 0.00 & 4.84 & 2.57 & 4.26 \\
\hline
\end{tabular}

Results show that $\mathrm{BF}$ is the variable with the highest average value, almost double that of PL, which is also twice as big as the results exhibited by BL and ST. These averages prove that BF is experiencing the biggest amount of change, followed by PL, BL and ST respectively. Consequently, BF and PL show more peaks (grey boxes in table 4-1) than BL and ST that both tend to change by smaller quantities and less frequently.

\subsubsection{Quantity of change in time: average rate of change of $B F, P L, B L$ and $S T$}

The objective of measuring the average rate of change in the elements of the urban landscape is to characterize discrete phases of spatial change.

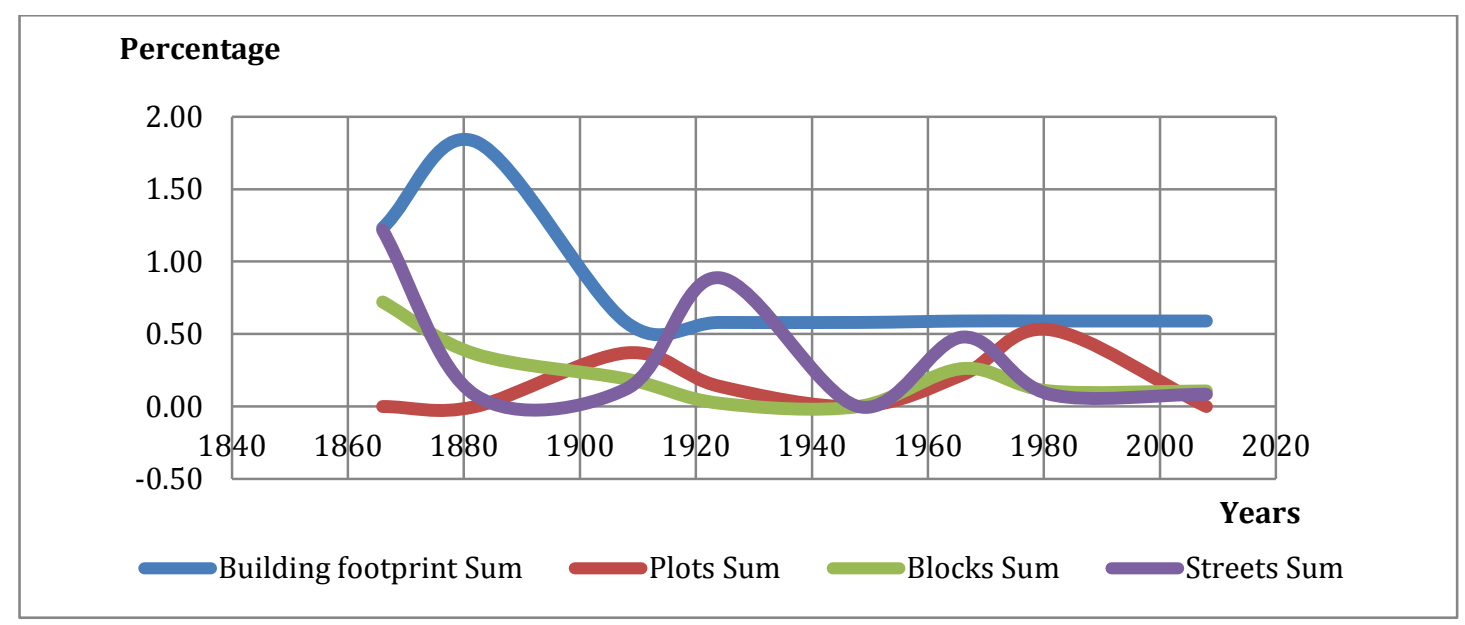

Figure 4-8. Sum average rate of change 


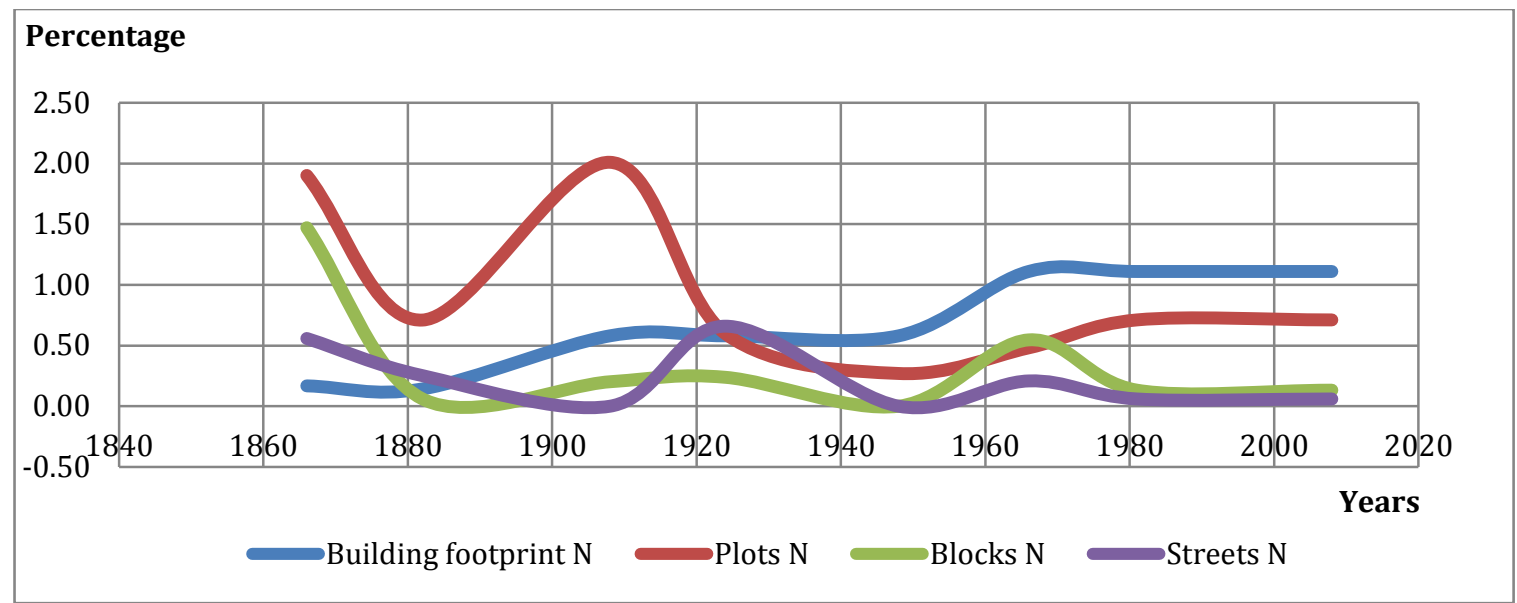

Figure 4-9. Number of features average rate of change

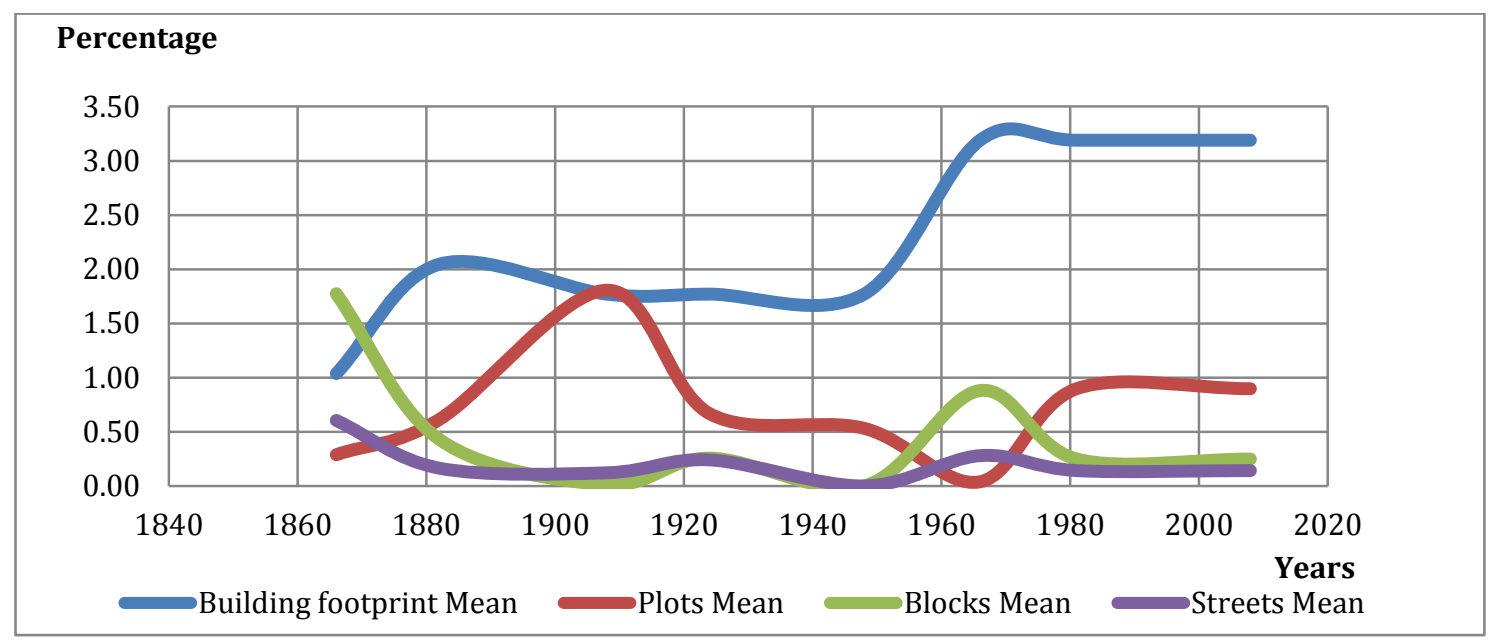

Figure 4-10. Mean average rate of change

Categorization of the quantity of change in time

Measurement of the different phases that characterize the speed of growth in the evolution of the urban landscape of Auckland CBD were established by defining the points where the average values within and across levels surpass the total average. The averages obtained in each period are based on the calculation of the average rate of change between periods. The rate of change shown is the average value of the period (Table 4-2). 
Table 4-2. Average rate of change in BF, PL, BL, ST (Time)

\begin{tabular}{|c|c|c|c|c|c|c|c|c|c|c|}
\hline & & $\begin{array}{l}1860- \\
1882\end{array}$ & $\begin{array}{l}1882- \\
1908\end{array}$ & $\begin{array}{l}1908- \\
1924 \\
\end{array}$ & $\begin{array}{l}1924- \\
1931 \\
\end{array}$ & $\begin{array}{l}\text { 1931- } \\
1948\end{array}$ & $\begin{array}{l}1948- \\
1966\end{array}$ & $\begin{array}{l}1966- \\
1981\end{array}$ & $\begin{array}{l}\text { 1981- } \\
2008\end{array}$ & Subt \\
\hline \multirow{4}{*}{$\mathrm{BF}$} & Mean & 1.04 & 2.05 & 1.77 & 1.77 & 1.77 & 3.19 & 3.19 & 3.19 & 2.25 \\
\hline & Sum & 1.23 & 1.83 & 0.58 & 0.58 & 0.58 & 0.59 & 0.59 & 0.59 & 0.82 \\
\hline & $\mathrm{N}$ & 0.17 & 0.14 & 0.58 & 0.58 & 0.58 & 1.11 & 1.11 & 1.11 & 0.67 \\
\hline & Aver & 0.81 & 1.34 & 0.98 & 0.98 & 0.98 & 1.63 & 1.63 & 1.63 & 1.25 \\
\hline \multirow{4}{*}{ PL } & Mean & 0.29 & 0.61 & 1.81 & 0.67 & 0.53 & 0.04 & 0.90 & 0.90 & 0.72 \\
\hline & $\mathrm{N}$ & 1.90 & 0.71 & 2.01 & 0.60 & 0.27 & 0.48 & 0.71 & 0.71 & 0.92 \\
\hline & Sum & 0.00 & 0.00 & 0.37 & 0.14 & 0.00 & 0.22 & 0.53 & 0.00 & 0.16 \\
\hline & Aver & 0.73 & 0.44 & 1.40 & 0.47 & 0.27 & 0.25 & 0.71 & 0.54 & 0.60 \\
\hline \multirow{4}{*}{ BL } & Mean & 1.77 & 0.43 & 0.01 & 0.26 & 0.00 & 0.88 & 0.25 & 0.25 & 0.48 \\
\hline & Sum & 0.72 & 0.36 & 0.19 & 0.02 & 0.00 & 0.26 & 0.11 & 0.11 & 0.22 \\
\hline & $\mathrm{N}$ & 1.47 & 0.06 & 0.20 & 0.24 & 0.00 & 0.55 & 0.13 & 0.13 & 0.35 \\
\hline & Aver & 1.32 & 0.28 & 0.13 & 0.17 & 0.00 & 0.56 & 0.16 & 0.16 & 0.35 \\
\hline \multirow{4}{*}{ ST } & Mean & 0.60 & 0.16 & 0.12 & 0.24 & 0.00 & 0.28 & 0.14 & 0.14 & 0.21 \\
\hline & Sum & 1.22 & 0.08 & 0.12 & 0.89 & 0.00 & 0.48 & 0.08 & 0.08 & 0.37 \\
\hline & $\mathrm{N}$ & 0.56 & 0.25 & 0.00 & 0.66 & 0.00 & 0.21 & 0.06 & 0.06 & 0.22 \\
\hline & Aver & 0.79 & 0.16 & 0.08 & 0.60 & 0.00 & 0.32 & 0.10 & 0.10 & 0.27 \\
\hline
\end{tabular}

Every level changes at its own rate and has its own higher and lower points that emerge at different moments in the evolution, with neither coincident periods nor constant or regular values across scales. Looking at the dynamics across scales, the values in the period from 1931 to 1948 do not show any value that surpasses the average for any level or variable, meanwhile the periods from 1860 to1882 and from 1948 to 1966 are both periods with a greater number of higher speeds of change at different levels. The remaining periods contain only one peak across levels. For the dynamics within scales, in relationship to the frequency of higher speeds of change, BF shows a major number of periods with values above average (3), implying that the frequency of change is faster at this level. This fact can be corroborated in the total average of the level. The results demonstrate that PL change faster than BL and ST (Table 4-2). At the same time BL change faster than ST, however they experience a lesser quantity of fast periods but more intense change, especially in the period from 1860 to 1882 . Basically, BL and ST performed in a similar way, with an initial period of fast change followed by a second period of constant speed (Table 4-2). This behaviour can be linked with changes in the structure of the landscape when Auckland passed from being a walkable city to a motorized city (see Appendix 1). 


\subsubsection{Synthesis of the quantity and speed of change}

In order to make a diagnosis of the quantity of change in the evolution of $\mathrm{BF}, \mathrm{BL}, \mathrm{PL}$ and ST, the information obtained in the analysis of the quantity of change in space and time was joined together in one table. The objective was to test if it is possible to merge change in time and space in a coherent phase. The goal was achieved by superimposing parallel timelines with the global averages of each dimension and by grouping and discriminating phases with big and small values. A pertinent observation is that $\mathrm{BF}$ has more coincident phases because some of the periods were averaged due to the lack of information in the available maps.

Table 4-3. Quantity and speed of change in BF, PL, BL, ST

$\begin{array}{lllllllll}1866 & 1882 & 1908 & 1924 & 1931 & 1948 & 1966 & 1981 & 2008\end{array}$

\begin{tabular}{|c|c|c|c|c|c|c|c|c|}
\hline \multirow[b]{4}{*}{$\mathrm{BF}$} & \multicolumn{2}{|l|}{ P1 big change } & \multicolumn{2}{|c|}{ P2 small change } & \multicolumn{4}{|c|}{ P3 big change } \\
\hline & 12.99 & 34.81 & 15.63 & 6.84 & 15.63 & $\begin{array}{l}25.7 \\
2 \\
\end{array}$ & 24.45 & 36.32 \\
\hline & \multicolumn{2}{|l|}{ P1 fast change } & \multicolumn{2}{|c|}{ P2 slow change } & \multicolumn{4}{|c|}{ P3 fast change } \\
\hline & 0.81 & 1.34 & 0.98 & 0.98 & 0.98 & 1.63 & 1.63 & 1.63 \\
\hline \multirow[b]{4}{*}{ PL } & \multicolumn{3}{|l|}{ P1 big change } & \multicolumn{3}{|c|}{ P2 small change } & \multicolumn{2}{|c|}{ P3 big change } \\
\hline & 23.68 & 11.47 & $\begin{array}{l}22.3 \\
5\end{array}$ & 3.28 & 4.56 & 5.61 & 5.26 & 14.47 \\
\hline & \multicolumn{3}{|l|}{ P1 fast change } & \multicolumn{2}{|c|}{ P2 slow change } & \multicolumn{2}{|c|}{$\begin{array}{ll}\text { P3 fast } \\
\text { change }\end{array}$} & P4 slow change \\
\hline & 0.73 & 0.44 & 1.40 & 0.47 & 0.27 & 0.25 & 0.71 & 0.54 \\
\hline \multirow[b]{4}{*}{$\mathrm{BL}$} & \multicolumn{2}{|l|}{ P1 big change } & \multicolumn{3}{|c|}{ P2 small change } & \multicolumn{2}{|c|}{$\begin{array}{ll}\text { P3 big } \\
\text { change }\end{array}$} & P4 small change \\
\hline & 21.15 & 7.40 & 2.15 & 1.21 & 0.16 & 0.00 & 8.44 & 4.39 \\
\hline & P1 fast change & \multicolumn{3}{|c|}{ P2 slow change } & \multicolumn{2}{|c|}{$\begin{array}{ll}\text { P3 fast } \\
\text { change }\end{array}$} & \multicolumn{2}{|c|}{ P4 slow change } \\
\hline & 1.32 & 0.28 & 0.13 & 0.17 & 0.00 & 0.56 & 0.16 & 0.16 \\
\hline \multirow[b]{4}{*}{ ST } & \multicolumn{2}{|l|}{ P1 big change } & \multicolumn{3}{|c|}{ P2 small change } & \multicolumn{2}{|c|}{$\begin{array}{ll}\begin{array}{l}\text { P3 big } \\
\text { change }\end{array} & \\
\end{array}$} & P4 small change \\
\hline & 12.69 & 4.28 & 1.30 & 4.17 & 4.23 & 0.00 & 4.84 & 2.57 \\
\hline & P1 fast change & $\begin{array}{l}\text { P2 slow } \\
\text { change }\end{array}$ & \multicolumn{4}{|c|}{ P3 fast change } & \multicolumn{2}{|c|}{ P4 slow change } \\
\hline & 0.79 & 0.16 & 0.08 & 0.60 & 0.00 & 0.32 & 0.10 & 0.10 \\
\hline
\end{tabular}

A pattern that can be inferred from the table is that the parallel between changes in time and space tends to be less coherent at levels where both changes in time and space are less intense. In consequence the characterization of phases in BF is more consistent than the description of phases in BL and ST. 
Table 4-4. Diagnosis of quantity and speed of change

1866

1908

1948

1981

2008

BF

P1 big/fast change

P2 small/slow change

P3 big/fast change

PL

P1 big/fast change

P2 small / slow-fast change

P3 big/ fast-slow change

BL

\begin{tabular}{|l|l|l|l|}
\hline P1 big/ fast-slow change & P2 small/slow change & P3 big / fast-slow change & $\begin{array}{l}\text { P4 small/slow } \\
\text { change }\end{array}$ \\
\hline
\end{tabular}

ST

\section{P1 big/ fast-slow
.5. Conclusion}

Every element of the urban landscape evolves in a discrete way, an affirmation that can be checked by looking at the amount of change that every element has exhibited in time and space. Therefore, the analysis of only one element of the structure of the urban landscape does not describe the dynamics of change of the whole system, proving that urban landscapes are complex entities.

The evolution of BF, BL, PL and ST is characterized by discrete rates of change in quantity and speed that are linked with their sizes. Small elements tend to change more and faster than big elements that change less and slowly. BF and PL tend to experience bigger and faster change than BL and ST. This relationship can be checked through the average change in sum and number and also in the average rate of change, where BF always has the biggest values and PL the second largest, followed by BL and ST. As a corollary, the number of features in each element is reversibly proportional to its size (Fig. 4-2). For this reason, the quantity of big elements present in the urban landscape is less than the quantity of smaller elements. This relationship between size and number of BF, PL, BL and ST within the texture of the built environment reveals the presence of a hierarchy of elements in the urban landscape that can be compared with the Panarchy (see section 2.3.6) and understood as an evidence for the existence of an urban Panarchy (see section 3.3). 

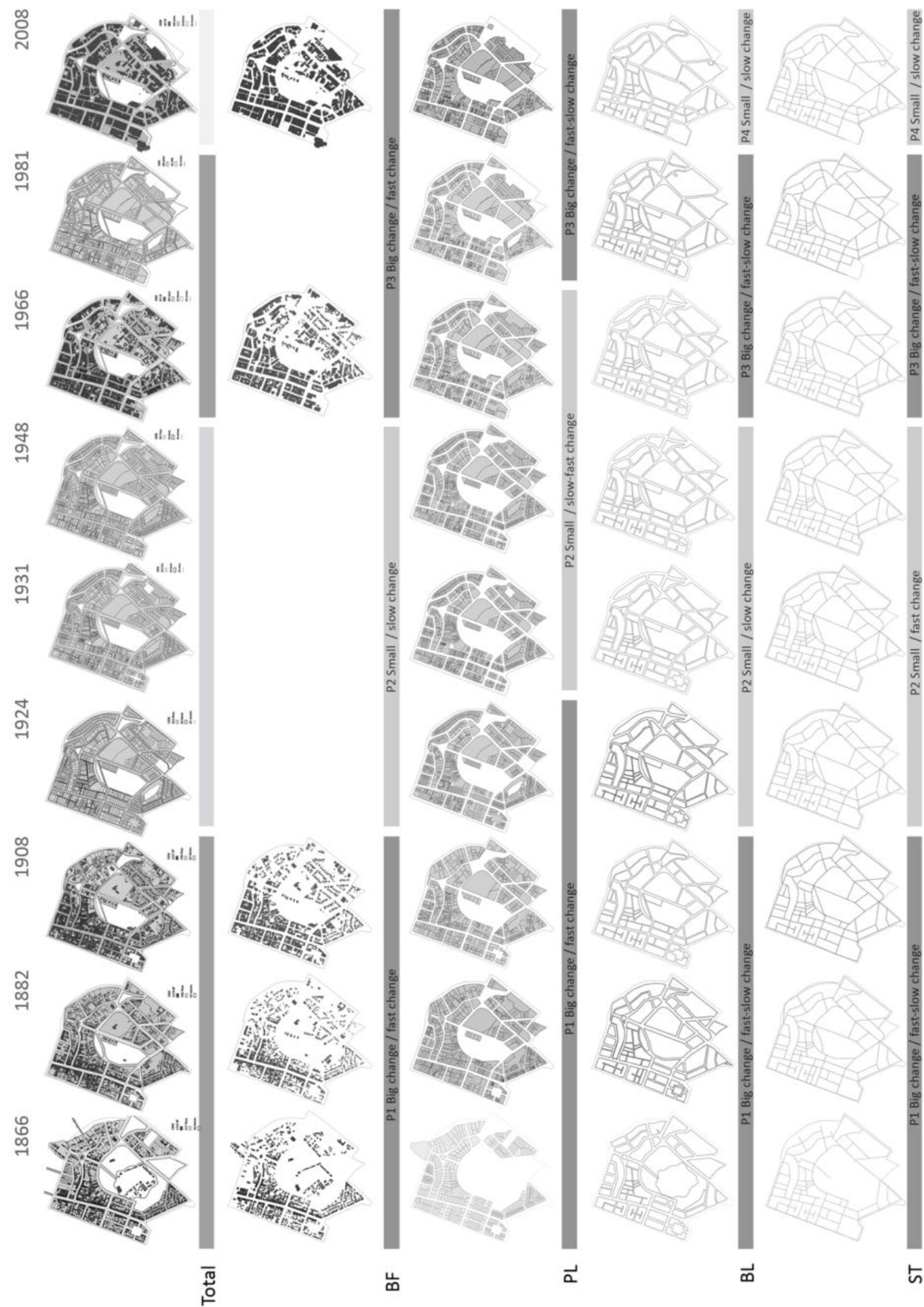

偻

岗
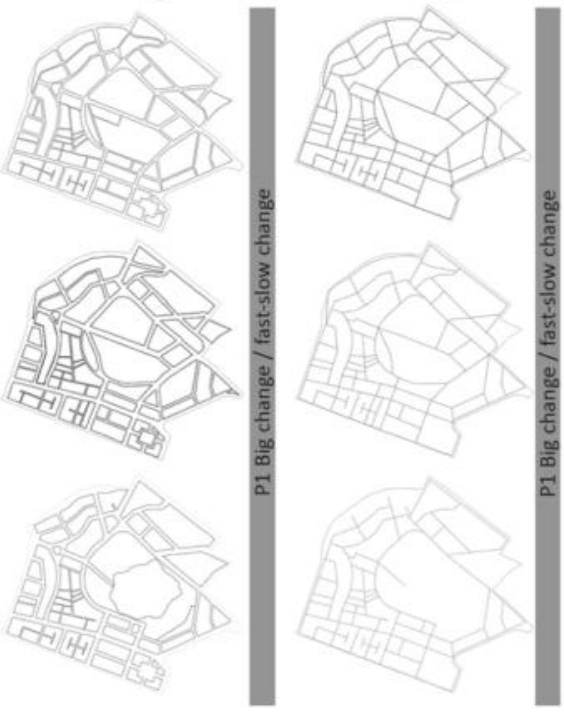

$\vec{\omega}$

เ 
The importance of developing a quantitative historical analysis of an urban landscape is that it permits the building of a framework and provides a reference for the future management of the system by evaluating the same system. The idea of understanding the structure of urban landscapes as a Panarchy is useful for measuring the evolution of urban landscapes and for making analyses at different scales.

Phases obtained in the analysis reveal an alternative history of the urban landscape, one that is told from the internal evolution of its structural elements. The kind of history that a Panarchy reveals is multiple and complex because it involves different scales or levels, discrete variables, and alternative domains of space and time.

This history of the urban landscape of the east side of Auckland CBD, when it is analysed as a Panarchy, shows that events and processes tend to affect the elements of the urban landscape in discrete ways. Consequently, a non-linear performance at every level of the structure (BF, PL, BL, ST) arises. The non-linear performance can be corroborated by observing the cyclical behaviour that every level shows through different phases.

By assessing evolutionary dynamics in an urban landscape it is possible to understand the rhythm and cycles of change in a built environment, a factor that is essential in the theoretical framework of ecological resilience when trying to frame the analysis of change through the adaptive cycle metaphor.

The method of analysing the evolution of an urban landscape by measuring change in its townscape through historical maps is time consuming work. This factor needs to be taken into account before starting any urban morphological analysis. From the research of historical maps to the digitalization process of the information and its later development, the analysis should be by a group of people instead of only one researcher.

The accuracy of the information can be improved by finding more maps at different periods so the breaks in information can be overcome. Another way of improving the precision is investing more time and resources in the drawing process. The quality of the maps also plays an important factor but even in these conditions, the act of bounding and defining the perimeter of BF and PL limits in each period is hard without having the certainty contained in original documents 
of each property. Finally, the analysis is only focussed on the two-dimensional properties of urban landscapes, ignoring townscape, and therefore it does not represent the whole three-dimensional urban landscape.

The definition of the scales was theoretically framed in relationship with the resistance to change that every element of the urban landscape exhibits but the level of resolution of the analysis remains constant. However it could be possible to use the same method of analysis by defining discrete geographical scales within the boundaries of a case study, for example, measuring the dynamics of change in one street, then in a neighbourhood, and at the end in the whole or part of the city. Another possibility is to apply a Conzenian approach to defining different levels of complexities in one case study by analyzing morphological units, plan units and regions.

One thing that was not observed in the present analysis is the theoretical assumption taken in ecological resilience where slow variables entrain fast variables in a slaving process, implying that small change in slow variables will provoke big change in fast variables. It could be interesting to investigate if the relationship between size, speed and quantity of change can be also observed within the scale of every element of the urban landscape.

Another important research investigation could be conducted by analyzing if changes in slow variables like ST or BL, entrain fast variables like BF and PL. 


\section{Resilient assemblages: identity and resilience}

\subsection{Introduction}

This thesis uses the concept of ecological resilience as the capacity of complex adaptive systems, like ecosystems or cities, to adapt to unpredictable change without losing their identity (Alberti \& Marzluff, 2004). Identity in this context refers to a threshold in the equilibrium state of a system. The persistence of the identity of a system implies that its structure (Panarchy) has buffered changes in order to stay in the same equilibrium state. If the theoretical framework of ecological resilience can be applicable to the analysis of urban landscapes, the identity of a place should behave in a similar way when changes affect the stability of the elements of its built environment.

The research investigates the resilience capacity of Nezu to two major fires and an earthquake in Tokyo: the Great Kanto earthquake and subsequent conflagrations (1923) and the World War II fire bombings (1945). The methodology uses the theoretical framework of ecological resilience and morphogenetics to measure change in the built environment of the chosen precincts before and after a crisis. The purpose is to analyze transformations in the built environment and their impact on the change or persistence of the identity of shitamachi areas.

The intention of this chapter is to test whether the concept of identity can be used in the analysis of the built environment as a reference to measure change in the stability of urban landscapes. In order to accomplish this goal it is proposed to measure the relationship between the persistence of an identity in a specific built environment, in this case the shitamachi 'description', and change in the elements of the urban landscape of Nezu.

If the identity of place can be used in urban landscapes to analyze the stability states of a system, it follows that changes in the elements of the built environment of Nezu (BF, BL, PL, ST) may not necessarily produce the same changes in the structure of its urban landscape. This means that the elements of the built environment of Nezu (BF, BL, PL, ST) can change through time, before 
and after crises (fires in the case of Nezu), but a robust resilience capacity will tend to keep the identity of the place fairly stable.

Results of the analysis will contribute to confirming or questioning the hypothesis about the dynamics of change of urban landscapes as set out in chapter 1 (see section 1.7), particularly the proposal that at the face of disturbances happening in the built environment, the resilience capacity of the urban landscape will try to maintain the identity of the system by keeping its structure fairly stable at larger scales while allowing variability and change at smaller scales (Holling, 1987). By doing this, the chapter tries to show evidence of how to apply ecological resilience to the analysis of urban landscapes.

\subsection{Structure of the chapter}

The chapter is divided into two studies linking changes in the built environment with persistence of identity.

The first study compares persistence in the identity of a built environment (Nezu) against change in the elements of the urban landscape. The objective is to show what the role of the urban landscape in the persistence of the identity of Nezu is, and particularly how the complexity of the urban landscape, through its multiple scales, is linked with its resilience capacity. The analysis is carried out by measuring morphological changes in sizes of blocks, streets and plots.

The second study observes changes in a characteristic system of spaces in the urban landscape of Nezu, the public-private interfaces, against change in the structure of its urban landscape. The objective is to show that the complexity of the urban landscape builds a quality that enhances the resilience of the built environment to changes in its urban landscape. According to the theoretical frameworks of assemblage and ecological resilience, the complexity, multiplicity and non-linearity of the system processes and dynamics should be reflected in the heterogeneity and complexity of the landscape. Therefore, public-private interfaces should be discontinuous, multiple and diverse to add complexity and resilience to the built environment of Nezu.

In both cases the objective is to describe changes in the stability state of urban landscapes. The use of identity as an instrument to observe and to 
measure the resilience of an urban landscape to changes in the equilibrium state of its built environment is tested. The two studies used in this chapter could help to find evidence that urban landscapes produce patterns of aggregations and discontinuities that are related with the structure described in the urban Panarchy (see chapter 4). Moreover, it is argued that these patterns are linked with qualities of the built environment that can be observed in changes or persistence in the identity of the place.

\subsection{Theoretical framework: resilient assemblages}

The complex dynamics of change in a built environment have an impact on the persistence of identity of place. Traditional philosophical approaches to identity of place focus only on elements of the urban landscape that linger, neglecting the processes of change that facilitate the persistence of some elements and the extinction of others. From this point of view every time that major changes affects the persistence of particular elements in built environment, this will be reflected in a change in the identity of the place. However, the same dynamics of change can be questioned if assemblage and resilience theory are acknowledged.

A complex approach to urban landscapes is developed by analysing the identity of place as a resilient assemblage. The methodology uses the philosophical approach of assemblage to link theoretical backgrounds from philosophy, ecology and urban studies (see section 2.2.2). By using this approach places are analysed as complex adaptive systems (Levin, 1998). Identity is based upon multiplicity and is always present in a process of becoming (Dovey, 2010). In a similar manner to stability states implying a relationship between a system and its context, the concept of identity of place links individual elements of built environments and the development of the totality of an urban landscape. Identity is described in this research as a relational phenomenon that implies relationships between dynamics of change in individual objects and the whole system at the same time. The difference with traditional philosophical approaches is that changes in individual elements of a built environment will not necessarily produce the same quantity of changes in the identity of the place. 
Ecological resilience offers a theoretical framework for investigating the dynamics of change in systems and persistence at different scales and across scales (Gunderson \& Holling, 2002). The idea is that a complex system has the capacity to adapt to hazards because it operates by changing and adapting within and across scales. Therefore by reproducing the dynamics of change in the urban landscape of Nezu, within and across scales, it is possible to describe the influence of change in the persistence of the shitamachi identity (see section 5.3).

\subsection{Nezu and its identity}

Nezu is located in Bunkyo ward, one of the 23 central wards of Tokyo (Fig. 5-1). Nezu is dominantly a low rise, high-density residential area with ubiquitous greenery, and small local shops and galleries. It is located in a valley and is characterized as shitamachi (Low City). From its founding Edo has been divided in two regions: yamanote, the high city, and shitamachi, the low city. The shitamachi was characterized through the "mood of the Low City" (Seidensticker, 1983, p. 86) with its rows of wooden buildings and sense of neighbourhood community. The low city's grid was rigid, right angled, and small, tight, and cozy (Seidensticker, 1983). The intriguing fact about the evolution of the built environment of Nezu is that few buildings are preserved from the Edo period but its shitamachi character persists. One possible explanation is that the identity of the built environment of Nezu, based upon its shitamachi character, might be linked with the structure of the elements of the urban landscape and not only with its buildings (Muminovic et al., 2012a). 


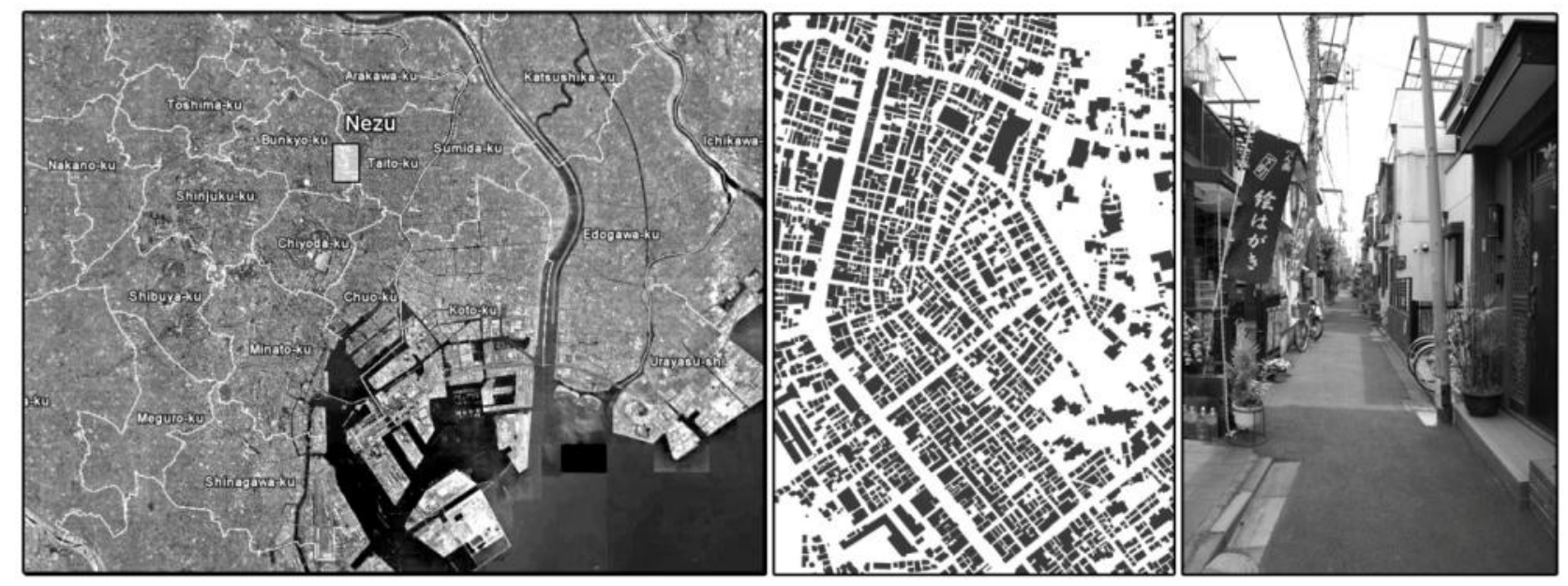

Figure 5-1. Map of Tokyo and Nezu (Muminovic et al., 2012a)

Nezu in Tokyo offers an opportunity to analyse the relationship between change and persistence in a built environment that has been evolving through time by changing and adapting its physical characteristics while conserving its identity. The fires are chosen because of their precise time and high degree of destruction as well as the possibilities they create for change in the physical setting of the place. One of the key characteristics of Tokyo lies in the numerous fires that have destroyed the city from the Edo period to modern Tokyo period (Seidensticker, 1983). Demonstrating the resilience capacity of the built environment to fires is an important factor in the persistence of the identity of place in Tokyo. The aim here is to analyse and develop an alternative understanding of the relationship between persistence of identity and change in the physical setting of a place by using a resilience and assemblage approach. Contrary to the timelines in chapter 4 that tend to map the general resilience of the system, here the timeline was used to map changes in relationship with only one threat, fires. In this way the study is narrowed to the analysis of the specific resilience of the system (B. Walker, 2007). 


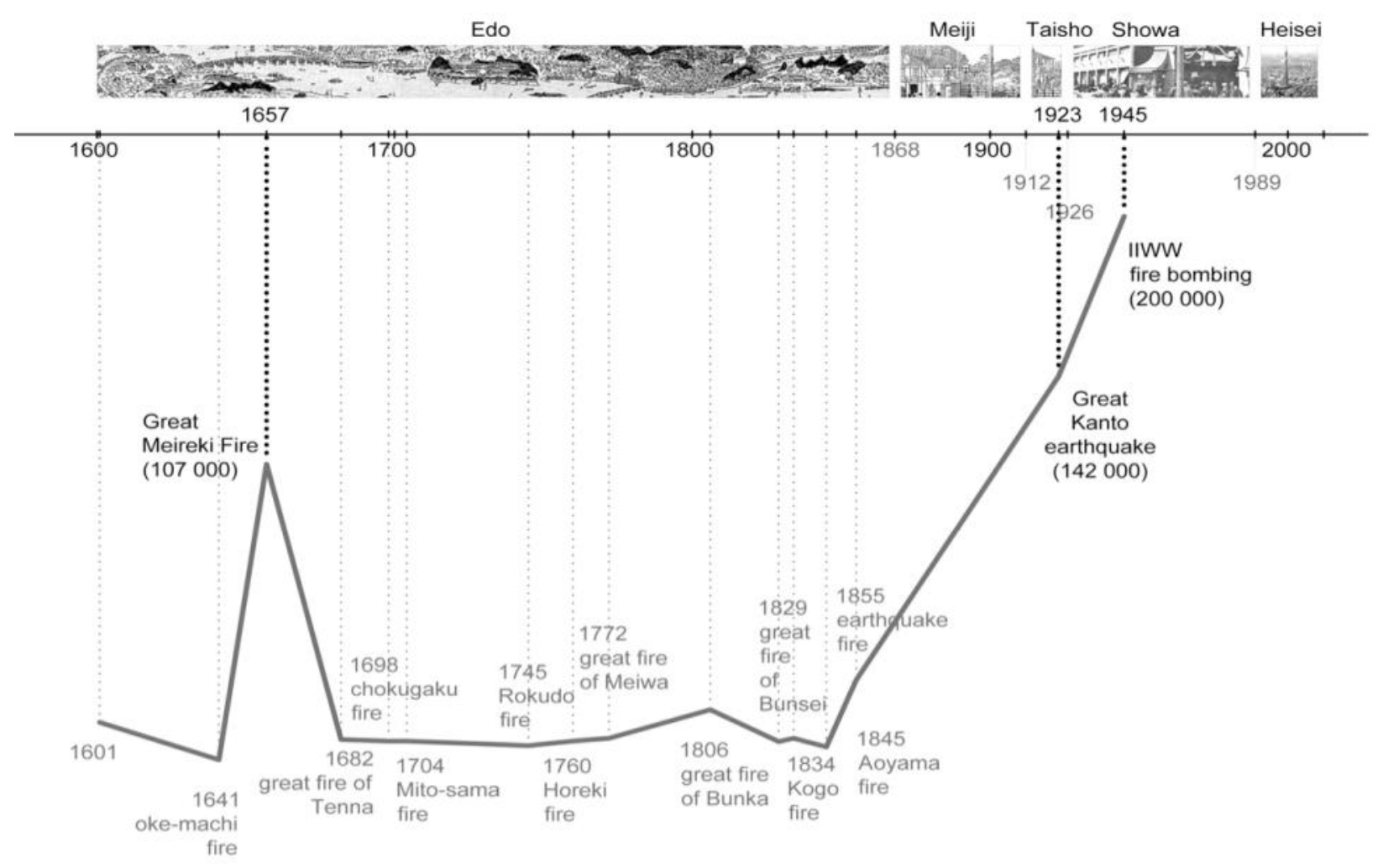

Figure 5-2. Major earthquakes and fires in Tokyo from Edo to Showa periods (1600 to 1945) (Muminovic et al., 2012a)

The timeline shows that earthquakes and fires are recurrent hazards in the system (Fig. 5-2). The Great Kanto earthquake in 1923 and the fire-bombing in 1945 are the most dramatic events with respective losses of 142,000 and up to 200,000 lives (Sorensen, 2002). The timeline highlights (continuous lines represents the quantity of lives lost in each fire) that fires were more common during the Edo period but less intense: on average there were major conflagrations every six years (McClain, Merriman, \& Ugawa, 1994). The end of the long Edo period almost matches the change in the frequency and intensity of fires. After Edo, hazards became less frequent but more intense and destructive. One interesting aspect of fires is that they are excellent examples of socioecological hazards, because they mix natural and social components in one event. 

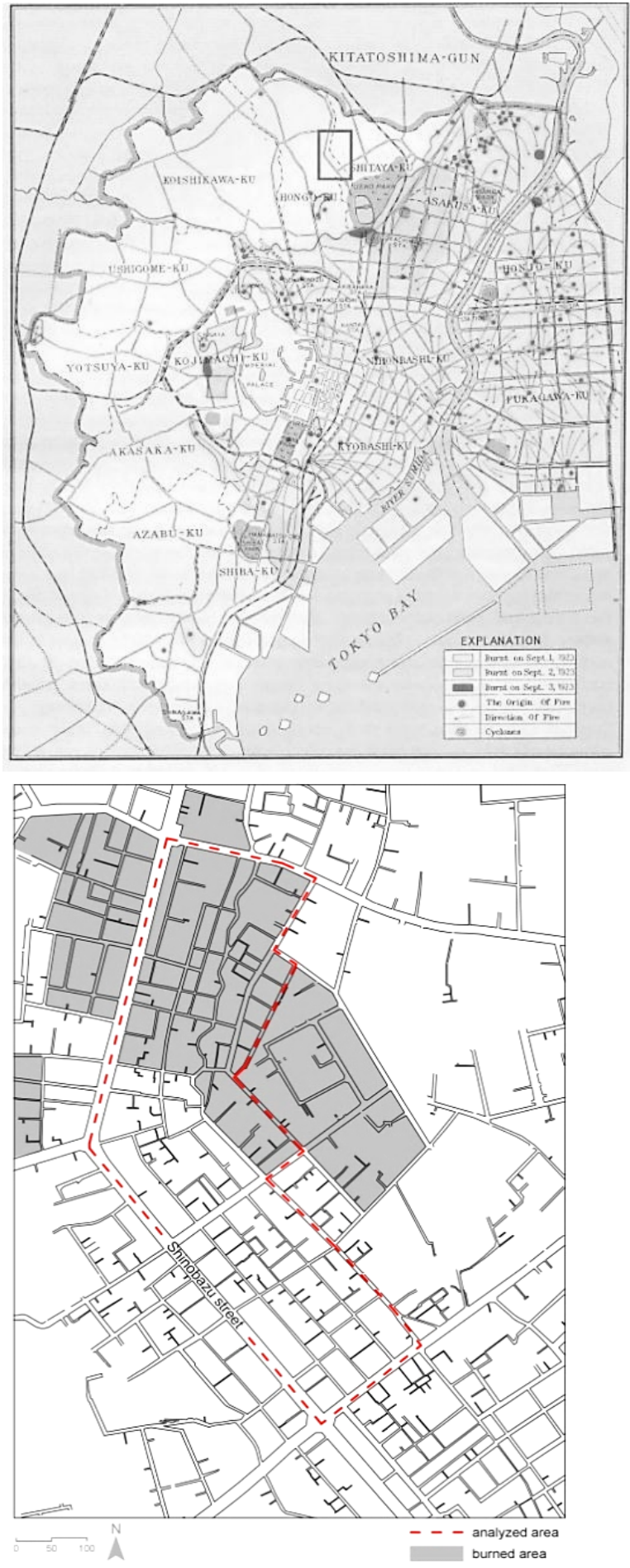

Figure 5-3. Map of burned areas in 1923 (left) and 1945 (right) (Anonymous, 1988; map, 1990)

The study area was chosen because its identity lingers from the past. It was not very affected by the conflagrations after the Great Kanto earthquake (1923) (in the map on the left in Fig. 5-3, Nezu is a rectangle that is outside the affected area) but was partly burned during the fire bombings of 1945 (Fig.5-3 
map on the left, burnt areas are in grey). For this analysis the chosen area has two discrete zones (Fig.5-3, red dotted lines). The northern part was burnt and highly impacted as a result (Area II) while the southern zone was not affected (Area I) (Fig. 5-3). Even though both zones have been impacted at different levels they maintain and share the same identity.

\subsection{Study 1: the role of the structure of urban landscapes in the resilience capacity of Nezu}

The initial assumption was that the persistence of the identity of the built environment of Nezu was enhanced by the resilience capacity of the structuring elements of its urban landscape, particularly the capability of the street system, block system and plot system to work at multiple equilibrium states, within and across scales, in order to buffer critical changes. In this research the interpretation of working in multiple stability states probably means that pressures and situations in the context of an urban landscape can change. As part of this some PL, BL, ST can be transformed or disappear, producing a disturbance in the equilibrium of the built environment, but the whole system will be able to absorb these changes by keeping the identity of the relationships between elements of the urban landscape more or less stable. Therefore the first step was directed to finding a way to characterize the interrelationships between BL, PL, ST, such that observations can be made by comparing alterations in structures and elements at the same time.

\subsubsection{Method for measuring morphological change in the urban landscape of Nezu}

For the purpose of this analysis the gathered data were old historical maps of this area from four different periods, giving information before and after the observed hazards. These maps belong to the period from 1888 until 1956. The maps are dated in four periods: 1888, 1919, 1923, and 1956. The maps used were at 1: 5000 scale. Maps are deriving from: cadastral maps (Uchiyama, 2011) maps of Edo to Tokyo periods (Takahashi, 1988) as well as Edit Committee documentation compilation maps. Collected maps (1888-1956) were scanned and redrawn as vector data using Auto Cad 2010 software. Moreover, in order to achieve a higher level of precision all maps were geo-referenced to the 
contemporary map of the same area using the ArcGIS 10 program and the data from Center for Spatial Information Science, The University of Tokyo. The following steps were then undertaken.

Morphological changes in blocks, streets and plots were quantified based on the size of each element of the built environment and calculated for each year and each group of elements (ST, BL, PL, BF) separately. Each element was named (see appendix 7) and quantified in terms of its respective size (area for BL, PL and BF and length for ST). Furthermore, standard deviation was calculated for each group of the elements and for each year separately in order to generate size classes. The calculation was conducted using Arc GIS 10 software and the number depends on the variability of the sizes of ST, BL, PL, and BF. The standard deviation was used as a parameter because it allows recognition of how far different groups of elements are from the average size of a variable (mean PL, BL, and ST). The predefined number of size classes was established as four because this was the minimum value always found in each BL, PL, and ST after different trials with alternative numbers of predefined outputs.

The degree of change was measured as the difference of size of each individual element of the built environment between two successive periods. These morphological changes were quantified as the percentage of the difference of the same element in two successive periods. The analysis showed that different complexities of data require different approaches in calculations of change.

The level of change for the blocks was based on the ratio of the area of one block to the whole area. The percentage of change was based on the ratio that one block has to the whole area of the same period (see appendix 8). In the case of subdivision of a block, the percentage of change was calculated for each subdivided block based on the whole size of the previous period. In the case of merging blocks the level of change was measured based on the ratio between the block with more similar characteristics of size to the new block. In the cases of similar sizes the block located at the centre of a new-formed block was chosen as the reference for change.

The degree of change of the streets was calculated based on their length. The degree of change of length is calculated based on the mid line of the streets. 
Streets were named and traced in all maps (see appendix 10). The change to the street is calculated based on the longer length without distinction of the nature of change, and whether the street is growing or shrinking. In the case of the appearance of a new street, the change was calculated as $100 \%$. In the case of the disappearance of a street, the street change is also calculated as $100 \%$ and shown on the map with a dashed line.

The calculation of level of change of the plots demanded a different approach. Due to the number and fact that plots are changing at a higher rate than blocks and streets the plots did not follow the same method of calculation of change of the area. Thus the degree of change was not calculated separately for each plot (see appendix 9). The first two periods were analyzed based on the data of the plots and the second two periods based on the analysis of building footprint. The first two periods define the change of the plots based on the size of all preserved plots in one block, represented at the level of block. Degree of change is defined as the relationship between sums of area of plots preserved in the block and the whole area of that block and is shown as a percentage. Blocks that are differently divided into plots are considered to have a degree of change of $100 \%$. In the third period (1932-1956) the degree of change was calculated based on the building footprint due to the lack of information about the plots. Degree of change was calculated using the existing information about the plots (map from 1932) and building footprint (map from 1956). In order to the define degree of change of each plot these two maps were overlapped and building footprint in 1956 was used to identify the plots that had not changed from 1932 The degree of change was calculated as the relationship between the sum of area of preserved plots within one block and the area of block. Degree of change was calculated as a percentage. Standard deviation is used in all periods in order to define the groups of different degree and diversity of change.

\subsubsection{Results from the analysis of morphological changes in sizes of blocks, streets and plots}

The first approach was characterized by mapping change in the size of BL, PL, and ST in order to observe relationships between bigger and smaller elements in each period and how they characterized different areas. 
The number of size-classes of BL was continuously increasing from 1888 to 1956. Areas I and II show a parallel phenomenon of intense subdivision of land from 1888 to 1919 along with a clustering in two areas. A re-organization in 1932 shows BL sizes more averaged and homogeneous. In 1956 the BL structure restarted a process of intense subdivision. Area II evolves from an organic distribution of BL to a more regular distribution pattern with a higher number of small size BL than Area I, which evolved by consolidating an orthogonal grid (Fig. 5-4).

The length of the ST from 1888 to 1956 shows a similar process of dispersion. The diversity of the street system increased from 1888 to 1919 and was characterized by the appearance of a network of medium and large size ST. The diversity achieved by the urban landscape is conserved until 1932. From 1932 to 1956 the size classes diminished and the street network became more homogeneous around medium and small sizes. The evolution of the street system in Area II experienced a change from a disconnected distribution in 1888 to a dense and clustered network in 1919. The network exhibits an agglomeration in its east side around 1956. In Area I the evolution took two paths: from 1888 to 1919 it developed in a north-south direction; from 1932 to 1956 new small ST increased the connectivity between west and east sides. The novelty is small dead end streets that appear in both areas (Fig. 5-4).

The plot evolution reveals a continuous process of subdivision of bigger PL into smaller ones in two phases: the first from 1888 to 1919, and the second from 1932 to 1956. Classes of size of PL are in all periods homogeneously dispersed in both Areas I and II. The map from 1956 shows higher diversity of classes of size at the level of BL, and less at the level of ST and PL. The maps from 1956 do not show the higher disturbance in the size classes of BL, ST and PL (Fig. 5-4). 


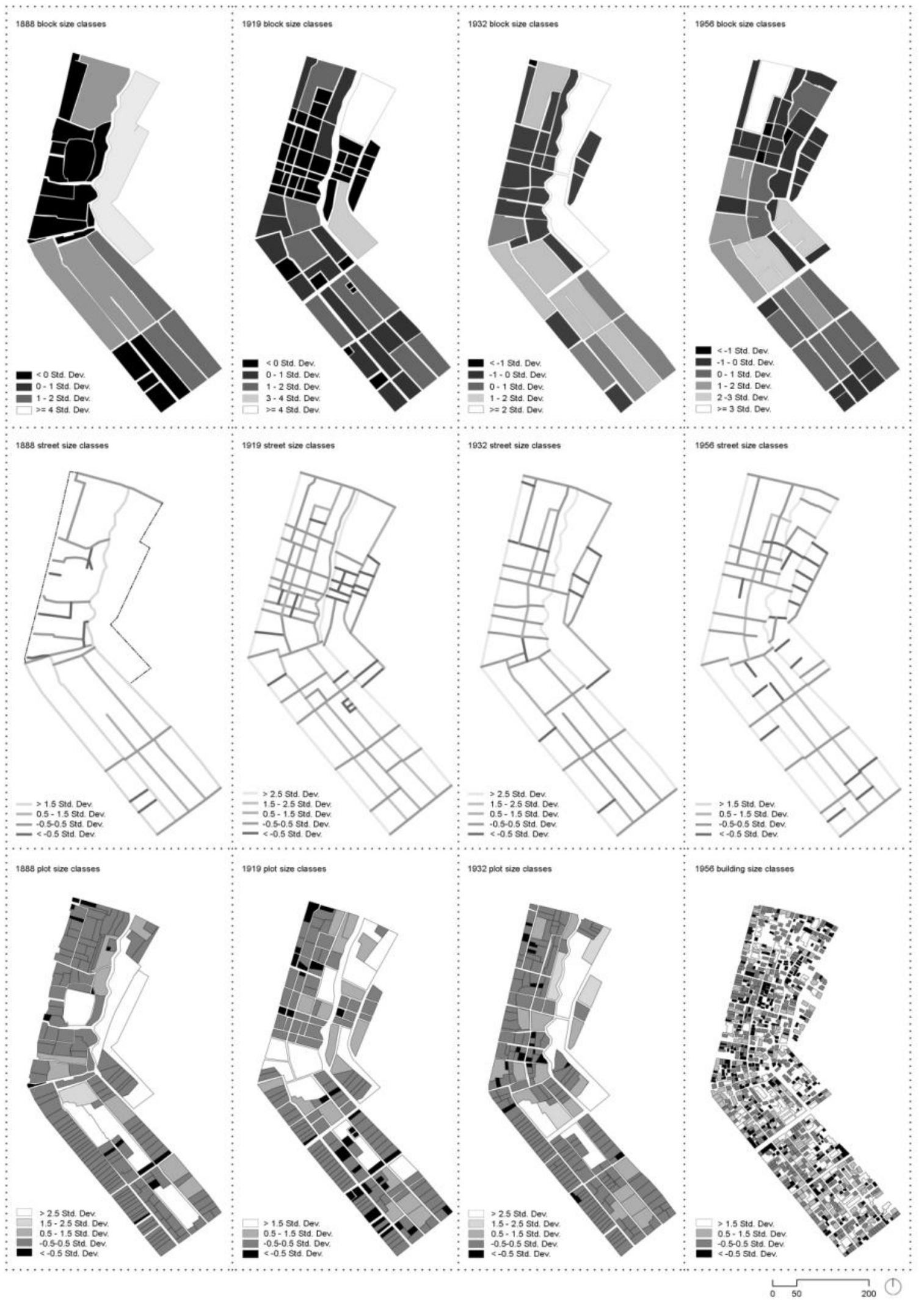

Figure 5-4. Size classes of blocks, streets and plots (top-bottom) 


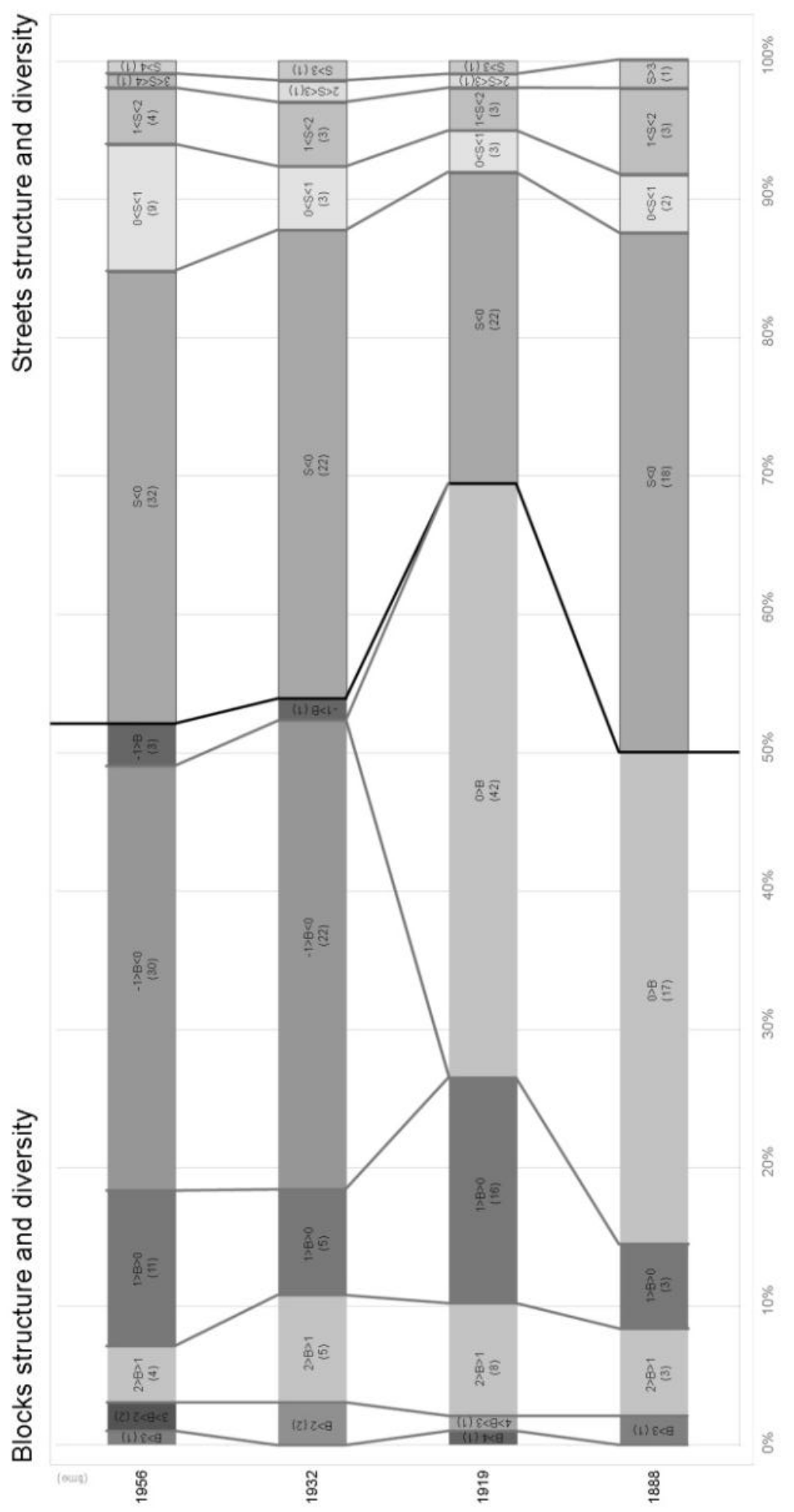

Figure 5-5. Diversity of blocks and streets through time 
The diagram of the evolution of different size-classes in BL and ST (Fig. 55) presents four horizontal bars corresponding to each period of the analysis. Each bar contains two elements, namely BL and ST that occupy one half of the diagram each and that are subdivided into a series of segments that represent size-classes in each category. In every segment the size range and the numbers of elements contained are described. The magnitude of every segment is in relationship to the percentage of elements confined for each size-class. In this way it is possible to have a panorama of the evolution of BL and ST and also observe how groups of similar BL and ST sizes were changing between periods, while inferring possible relationships between them.

During all periods the number of smaller elements in the size-classes of blocks and streets is high (Fig. 5-5). The diagram shows that the dynamic of change of BL and ST depends on the behaviour of every size class and the interaction between them. Change is produced in an independent way not only in the quantity of size classes in BL and ST but also in the percentage of elements contained in each size class. These dynamics expose non-linear behaviour and systemic interrelationships, both characteristics of complex systems (see section 2.2.1). The shifting diversity of size classes and elements in different periods could be evidence of adaptive processes.

\subsubsection{Results from the analysis of morphological transformations related with degree of change}

Area I shows a constant decrease in degree of change at the level of BL (from 60\% in 1919 to 34\% in 1956) (Fig.5-6). Area II demonstrates discontinuity in degree of change at the level of blocks (79\% in first period, 36\% in second period and $53 \%$ in third period). The degree of change of blocks shows constant decrease through time (Fig. 5-6). 


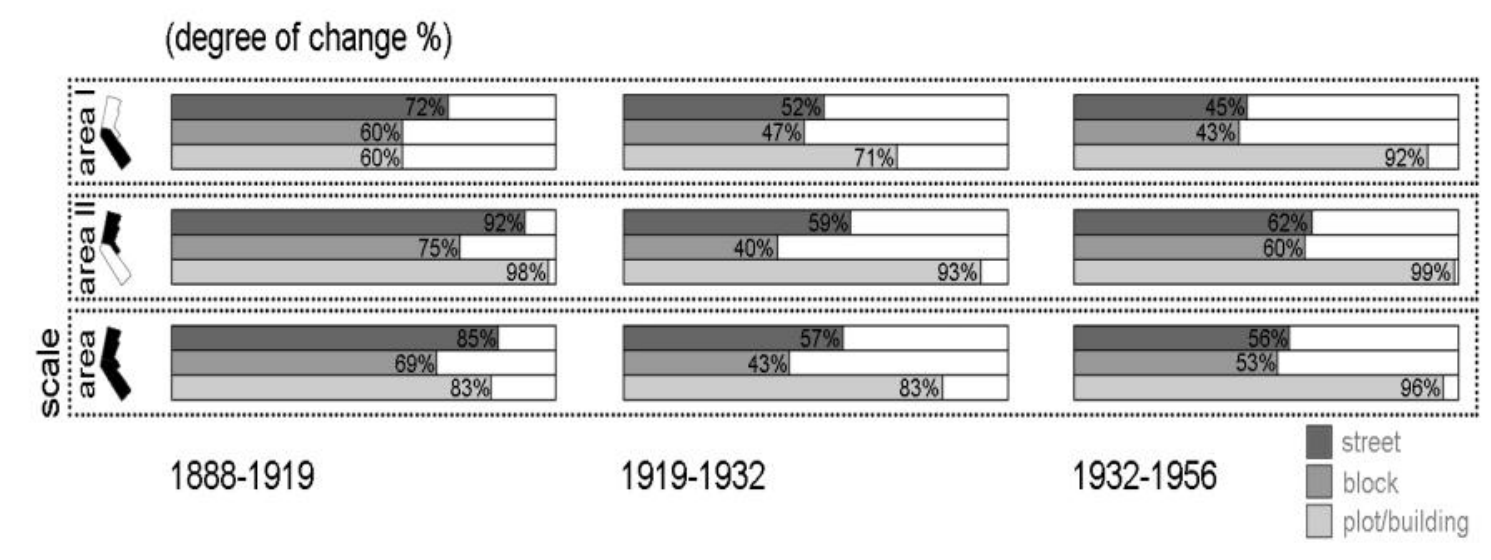

Figure 5-6. Average degree of change in blocks, streets and plots

Streets in Area I and the whole area show a decrease in the degree of change through time. The difference is high for the first two periods and low for the second showing the system is stabilizing (Fig 5-6). High intensity of change at the level of BL shows a tendency for clustering in Area II in the first period (1888-1919). In the same period Area I shows a more homogenized distribution of different intensities of change. Over time high intensity of change is changing its position and tends to be less clustered in both areas.

The degree of change in plots (Fig. 5-7) increases in areas where the subdivision of blocks and the appearance of new streets are also intense. The evolution of the intensity of change in PL shows a progressive expansion from the west to the east side in Area II, and a process of homogenization affecting all of Area II. The smaller BL, ST and PL are more numerous and frequent than their bigger counterparts. Based on this it is observed that ST and PL show a higher intensity of change than BL. The intensity of change of PL increases through time at all scales. The major intensity of change is linked with the areas where smaller sizes of BL cluster, while less intensity of change is linked with areas where the biggest sizes of BL are clustered (Fig. 5-7). 


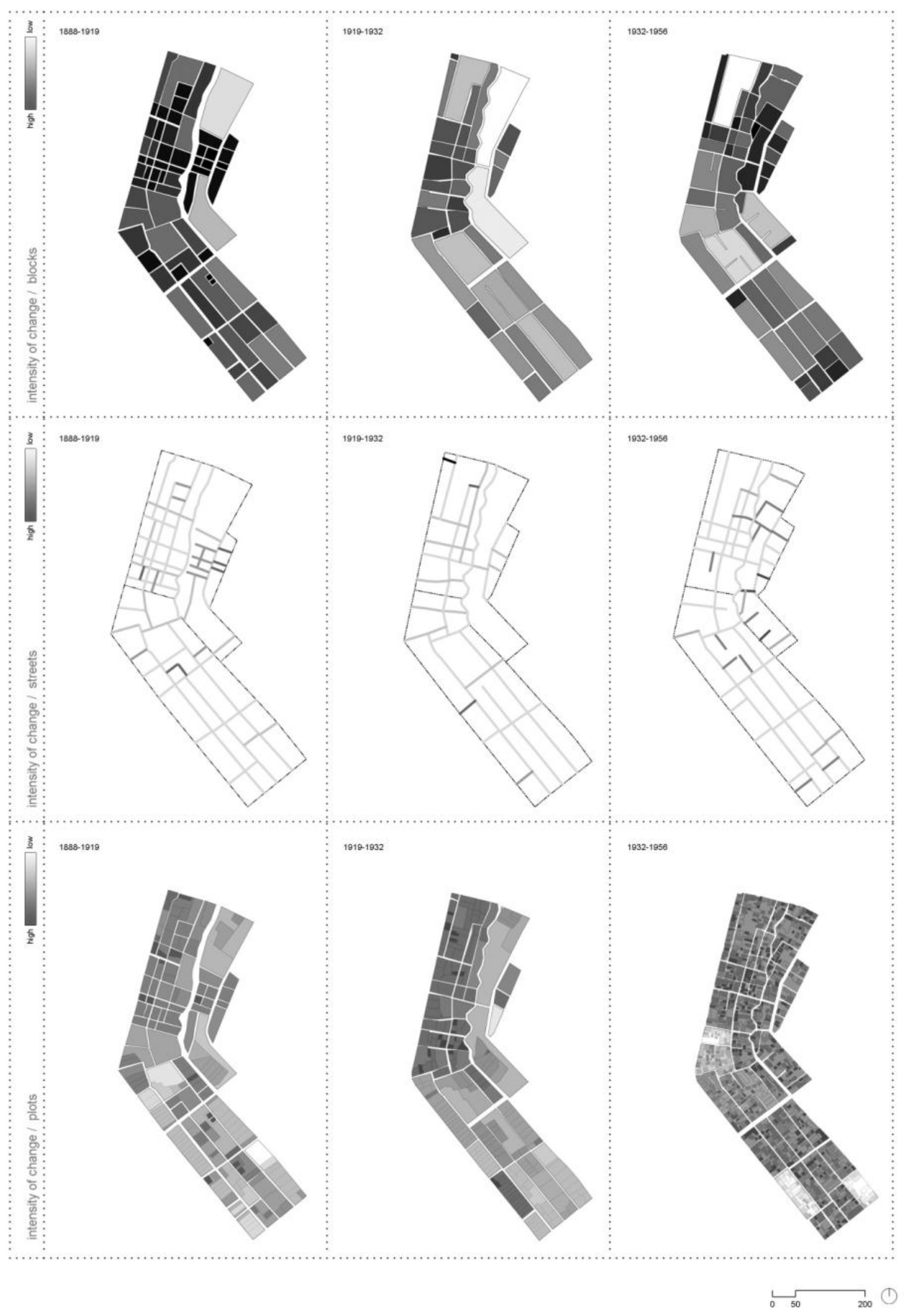

Figure 5-7. Intensity of change through time

134 
Results confirmed that Nezu showed resilience to the destruction caused by fire bombings after WWII. The persistence of the identity of shitamachi in $\mathrm{Nezu}$ is linked with the resilience of its physical setting, particularly the resilience of the structure of the urban landscape. The lingering of the character of the physical setting of Nezu was not provided by the permanence of particular buildings instead it was supported by the adaptive capacity of the inner structure of the urban landscape. The resilience capacity provided by the system of plots, blocks and streets demonstrates the potential of the urban landscape to be a complex adaptive system that behaves and responds at discrete rates of change within and across scales.

The adaptive capacity of the urban landscape is built upon the complexity of the place understood as an assemblage. This assemblage produces a discontinuous landscape. Discontinuities can be observed in the size and intensity of change of the main components (BL, PL, and ST) of the urban landscape of Nezu. The discontinuities in its landscape contribute to its resilience capacity. The results support the importance of relationships between elements of assemblage. The change in one group of elements is buffered within scales and different elements supporting the overall equilibrium of the urban landscape. The persistence of the identity of the urban landscape depends on a continuous process of change in order to adapt to external and internal demands on the systems.

\subsection{Study 2: the role of interfaces in the resilience capacity of the built environment of Nezu}

The second analysis explores the resilience of the identity of the urban landscape of Nezu to changes in its built environment and it was based on the idea that public-private interfaces can be key elements of the resilience of urban landscapes (see section 2.6).

Public-private interfaces in Japanese urban landscapes have been defined as soft boundaries that have contributed to the consolidation of the identity of old towns (Nakagawa, 2005). Shelton (1999) explains that the size and structure of the spaces between the private realm of the house and public realm of the street in shitamachi creates soft boundaries that are vibrant spaces. At the same time, 
the dynamic between public-private interfaces across scales defines new characteristics at each scale. One street can be considered as a public space at a small scale but as private at a larger scale (Shelton, 1999; Sorensen, 2002). This fluidity of public-private interfaces continues at the scale of the house (Daniels, 2010; Nakajima, 1996) and their connections and structures have been highlighted as characteristics that contribute to the creation of the identity of Tokyo (Kitayama, Tsukamoto, \& Nishizawa, 2010). Furthermore, Jinnai (1995) highlights the importance of the structure and size of public-private interfaces for the preservation of the character of some neighbourhoods in Tokyo. Another set of characteristics that contribute to the identity of shitamachi is related to the size of elements in the urban landscape. The smallness of all built environment elements represents a quality that has produced identity (Seidensticker, 1983). That smallness is widely stressed as a distinct character of precincts in contemporary Tokyo, having an important role in the creation of their identities (Radovic, 2008).

For all these reasons it is proposed that the analysis of public-private interfaces as discontinuities in the built environment of Nezu will provide important information about the complexity and resilience of its identity. This becomes the question of how the relationships between public-private interfaces within and across scales contribute to the complexity of the built environment of Nezu and at the same time to the resilience of its shitamachi identity.

\subsubsection{Method to finding discontinuities and aggregations through cluster analysis}

Following a morphogenetic approach, the elements of the urban landscape chosen for analysis are building footprints, blocks and streets (Conzen, 1960). By scale framing these elements (van Lieshout et al., 2011) into different units of analysis, three levels of spatio-temporal resolution were created (Cash et al., 2006; Wu \& Li, 2006): street (ST), block (BL) and building footprint (BF). Consequently, public-private interfaces are measured and grouped following these three scales.

Boundaries of public-private interfaces were defined using different criteria at each level. The building level uses the area between the street facade 
of the building and the street margin. These areas encompass space from the single step between the front door of a house and its street to larger transitions between buildings and streets. Even though these areas seem to be insignificant at city level, they are essentials at building level, becoming particularly important when considering that Nezu does not have sidewalks in its urban landscape. The block level uses the area that comprises all public-private interfaces of buildings within a block and its internal streets. At this level the universe of all small transitions builds a whole system that includes a set of internal streets whose level of privacy varies, and that characterize the urban landscape of Nezu. Finally, at the street level, public-private interfaces are defined by the area of each street along with the public-private interfaces of buildings within the street.

In order to investigate the presence of discontinuities in the urban landscape cluster analysis was conducted (Garmestani et al., 2005) using a Weka software data mining process (Witten et al., 2011). The data analysed are the area of buildings footprints, blocks, streets and their respective public-private interfaces (ppi) measured in $\mathrm{m}^{2}$. The cluster analysis was done using a training set option with the simple EM (expected maximization) class EM -I $100-\mathrm{N}-1$-M 1.0E-6 -S 100, where max. alterations equal to 100 , min. standard deviation equals to $1.0 \mathrm{E}-6$, the number of cluster equals to -1 , and the seed equals to 100 .

For the purpose of illustrating the interfaces between public and private within and across block, buildings and street levels, only the extreme values (minimum and maximum areas) for each cluster of each element were analysed. With the aim of showing interactions across levels, clusters were re-layered to identify spatial aggregations between them. 


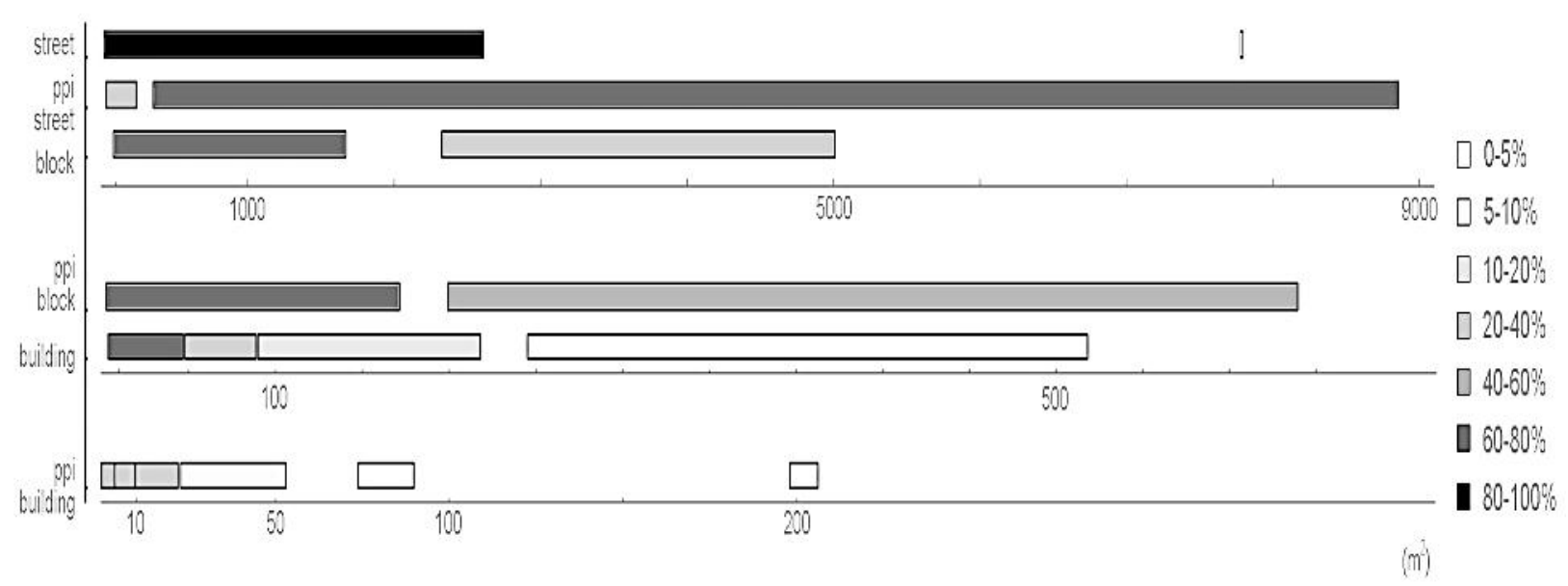

Figure 5-8. Aggregations and discontinuities in the structure of ST, BL and BF

The characteristics of two variables, public-private interface (ppi) and elements of the built environment (B, PL, and ST) show the existence of different levels and clusters within and across levels. The analysis of sizes of elements and public-private interfaces shows different numbers of clusters (bars in discrete percentage of dark in Fig. 5-8). Public-private interfaces show six clusters at the building level. Each level of block and street has two clusters. Elements of the built environment also show different numbers of clusters at different levels. The level of building has four clusters and each level of blocks and streets has two clusters. The clusters of smallest elements at each level show the higher numbers of elements. The numbers of clusters show that the smallest elements of both public-private interface and buildings have the largest impact on discontinuities and therefore on diversity of the built environment in Nezu. However, the results also show that sizes of discontinuities (gaps between grey bars in Fig. 5-8) are larger for bigger elements at each level and at higher levels (levels of blocks and streets). Larger discontinuities have significant influence on the diversity and complexity of a built environment. In these discontinuities, juxtaposed with the smallest elements, Deleuzian difference (Deleuze, 1994) becomes more palpable and therefore plays an important role in the complex identity of Nezu.

The aggregations of sizes of buildings and their public-private interfaces show a tendency to create clusters in the built landscape (Fig 5-9). The clusters in space become territorialized, having clear boundaries, when observed through relationships between building size and public-private interface. Namely, the 
clusters of sizes at the building level of both variables do not show any significant aggregation in space. However, overlapping of extreme values of public-private interface and size of elements of the built environment shows the creation of clusters in space (Fig. 5-9). These clusters have larger intensities of smallness and bigness and create discontinuities in the built environment of Nezu. 


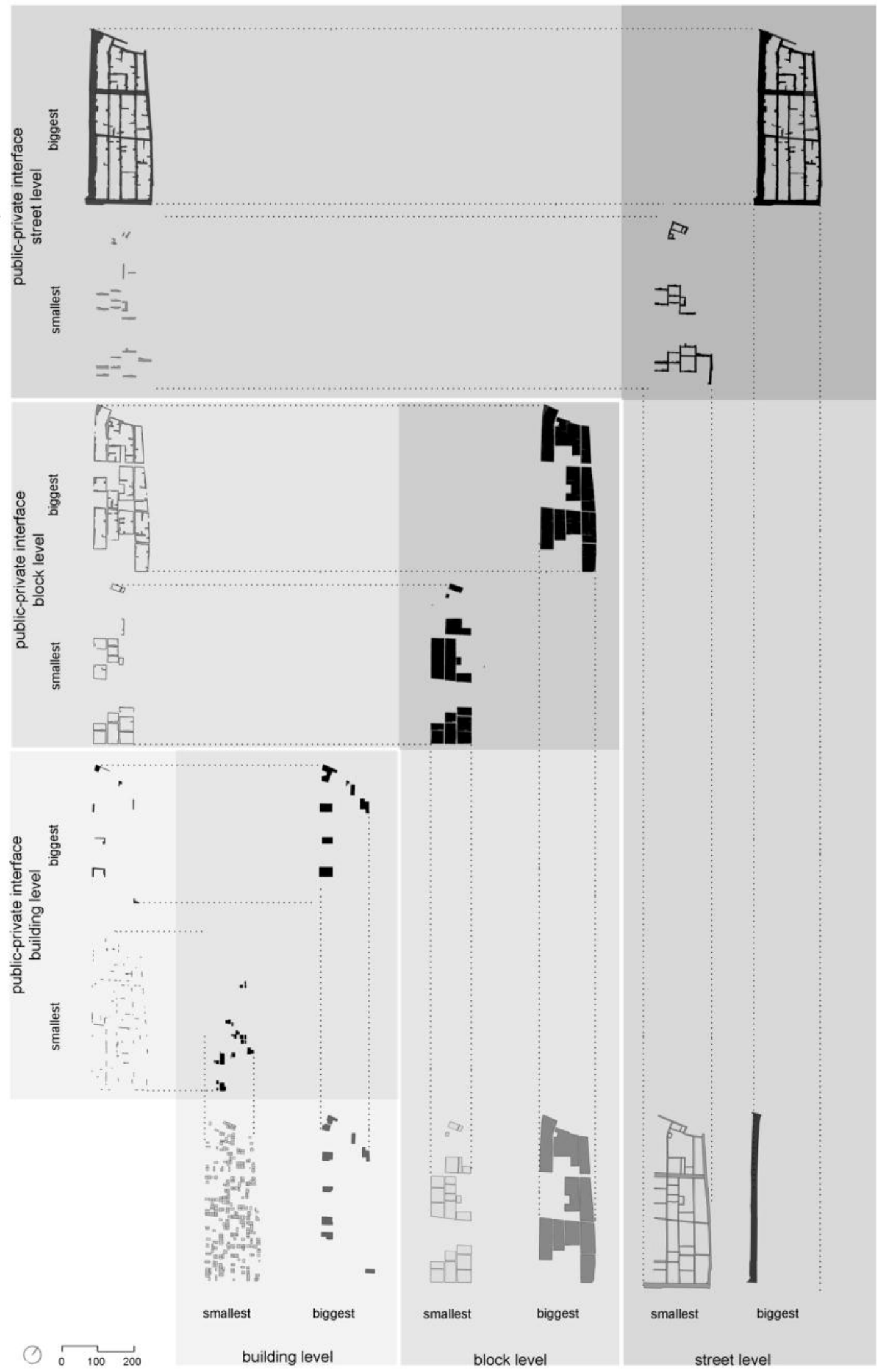

Figure 5-9. Clusters of public private interfaces at BF, BL and ST levels 
Aggregations and discontinuities of similar sizes of elements at different levels in the urban landscape create different qualities in the built environment landscape at each level, but a third kind of space also emerges when small clusters of elements are entrained by bigger clusters at a different level (Fig. 510). This characteristic, the diversity produced within each level and across levels contributes to the complexity of the built environment in Nezu.
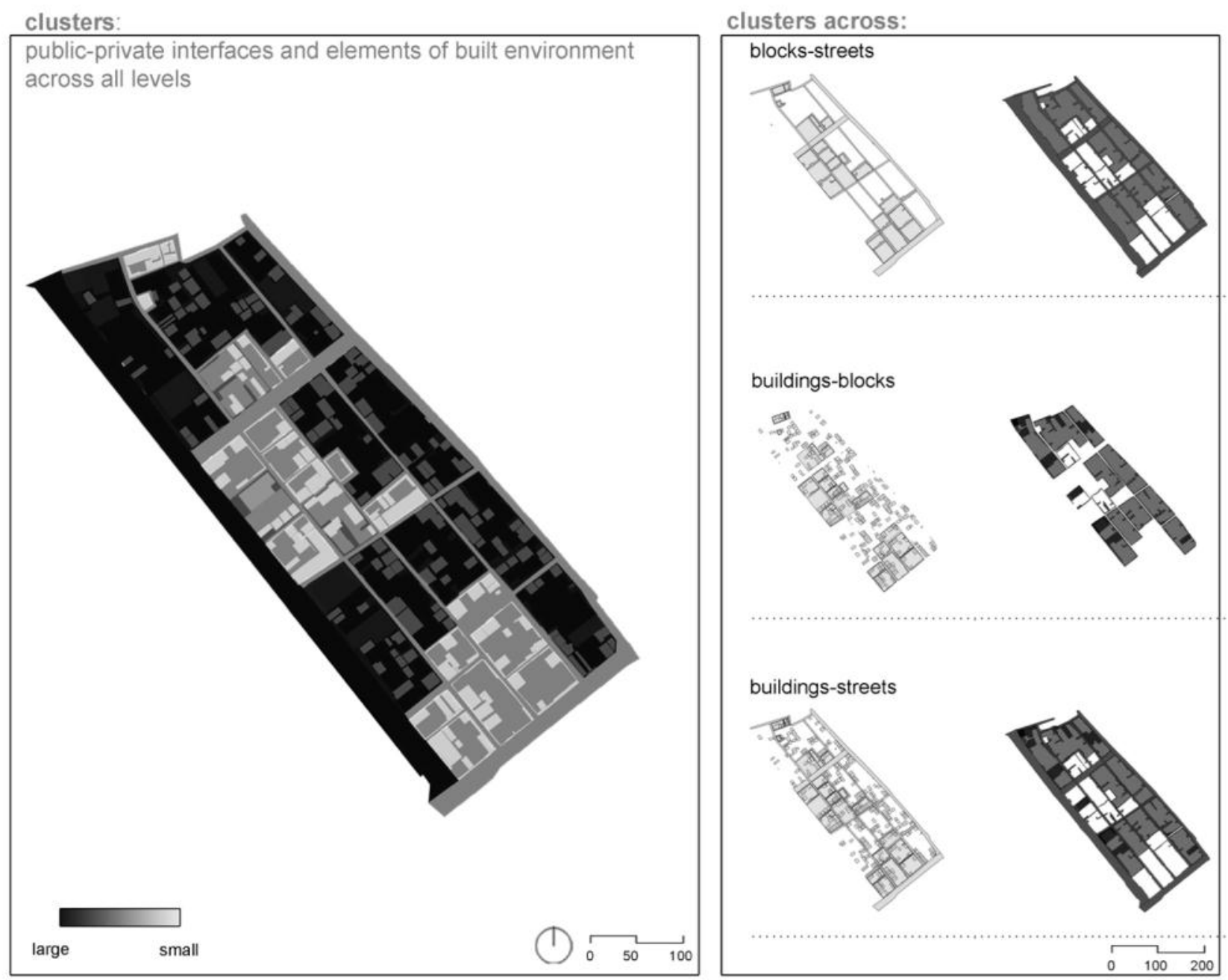

Figure 5-10. Clusters of public-private interfaces within and across BF, BL and ST levels

\subsection{Conclusions}

The analysis of the resilience of the identity of Nezu shows how the theoretical framework of ecological resilience can be applied to an analysis of the evolution of the built environment, in this case through the analysis of the 
stability state of the identity of a place. The analysis of the identity of Nezu using a resilient assemblage approach demonstrates that the use of the methodology and method proposed generate quantitative and qualitative information with which to understand the resilience of the built environment of urban landscapes in relationship to their complexity and adaptability.

The study shows that the persistence of the identity of a place can be linked with the resilience capacity of the structure of its urban landscape. Results have shown how different degrees of change have impact on the different elements of the urban landscape. However these changes did not produce critical changes in the identity of the place.

The example of Nezu shows that identity does not appear only as a product of otherness or difference defined as an external category. It is also produced through complex relationships within the built environment of place, in its discontinuity, diversity, multiplicity, or in other words, its complexity and adaptability.

The results show that complexity of the built environment in Nezu occurs at the smallest levels and in its smallest elements (Fig. 5-9). When connections between elements with the same characteristics happen within and across levels in Nezu, they produce a heterogeneous landscape (Fig. 5-10). The heterogeneity of the built environment of Nezu is supported by the presence of discontinuities that can be recognized in the appearance of levels and aggregations within each level (Fig. 5-8, Fig. 5-9). These discontinuities in the system and in the landscape have produced smallness at different levels (Fig. 5-9). This heterogeneity is expressed through zones of different intensities of smallness (Fig. 5-10). Since, the smallness is directly linked with the identity of Nezu, the results prove that complexity is an important state of its resilience. Furthermore, the analysis shows that relationships between elements of the built environment and publicprivate interfaces contribute to territorialisation of clusters and to intensification of the characteristics of smallness and bigness, creating the diverse urban landscape of Nezu. In addition, the results show that the presence of large elements represents an important part of complexity (Fig. 5-9). 
The resilience theory can be used to observe the evolution of the built environment in order to measure the performance of societies in an ecosystem. At the same time it provides a theoretical framework to illustrate the potential of assembling the equilibrium situation of a landscape with change and persistence in the identity of a place.

The analysis of Nezu was based on the fact that the place has managed to preserve its identity by changes in its built environment. Consequently, it can be inferred the place has held a certain resilience capacity. In order to have a comprehensive understanding of how certain characteristics of the built environment can contribute to the resilience capacity of the urban landscape it would be necessary to examine a place that has lost its identity in further research.

The challenge that resilience poses to understanding the identity of a place is the necessity to include the concept of reorganization as a dynamic of change in maintaining an identity by making it more adaptive. The analysis of the resilience of the identity of a built environment will open up new opportunities for comprehending how urban landscapes change in order to adapt and evolve.

Future studies with a resilient assemblage approach can be utilized to confirm whether the persistence of the identity of a built environment is linked with its high resilience capacity, or with its resistance to change. Another potential of the resilient assemblage approach for urban designers is the possibility of assessing the resilience of urban landscapes by observing changes in the identity of a place. The identity of a place can be used as a reference in order to analyse the stability and resilience of a built environment; therefore, identity should be rethought as an important concept for having insight into the evolutionary dynamics of urban landscapes. 


\section{Assessing and measuring resilience}

\subsection{Introduction}

The purpose of this chapter is to measure the relative resilience of two urban landscapes through the analysis of change in the heterogeneity of their built environments. This is felt to be useful not only for assessing the resilience capacity of two urban landscapes but also to try to see if certain kind of spaces, like green spaces, can be linked with these resilience dynamics of change. The analysis of interrelationships between the evolution of built environments and green spaces can produce knowledge about the resilience dynamics that make cities more adaptable to unpredictable change.

According to the hypothesis of this thesis, if the resilience theory can be applied to the study of urban landscapes, more heterogeneous built environments should have more resilience capacity to adapt to change, therefore they will change less over time. Following the initial hypothesis changes in the structure and diversity of the most heterogeneous urban landscape should not produce critical transformations in the structure of its green or built environment until its resilience capacity is surpassed. More heterogeneous urban landscapes should contains more heterogeneous built environments that at the same time should exhibit a higher resilience capacity, showing less quantity of change through time. Results from this chapter will contribute to finding more evidence about the resilience dynamics of change of urban landscapes and to showing a possible way of applying the resilience theoretical framework to the analysis of urban landscapes.

The chapter presents a comparative study of the resilience of the urban landscapes of the Auckland CBD in New Zealand and Nezu in Tokyo. The contrast between different evolutionary processes in each case study gives the possibility of testing the methodology and method applied in order to achieve its further external validation. The method integrates theoretical concepts from ecological resilience, assemblage and urban morphology (see section 2.2), and particularly a Conzenian approach (Whitehand, 2007). The method proposed assesses the persistence and change of aggregations and discontinuities in the structure of an 
urban landscape through time. Results should provide clues about the stability of the built environment and its resilience capacity.

The final aim of this chapter is to find evidence for applying the ecological resilience theoretical framework to the measurement of the resilience capacity of urban landscapes. This aligns with the goal of this research to propose a methodology and a method to assess the resilience of the built environment of urban landscapes while offering a theoretical framework for making a comparative analysis.

\subsection{Theoretical framework: Panarchy and the TDH}

The theoretical framework of ecological resilience proposed that complex adaptive systems, like ecosystems or urban landscapes, are driven by a small set of processes happening within and across scales in time and space (Gunderson \& Holling, 2002). Processes happening at the same scale of space and time tend to be clustered within the same scale and entrained by other processes at bigger scales. As a result, complex systems are organized in a dynamic hierarchy. When a system is at the face of unpredictable changes, the resilience of the hierarchy will tend to maintain patterns of distribution and organization of its elements, despite the loss of some of them (Gunderson \& Holling, 2002). If urban landscapes, as complex adaptive systems, are organized in the same way, elements of the built environment of Auckland and Nezu should be aggregated discontinuously in a dynamic hierarchy, generating different levels and processes within and across scales that will provide resilience to the system.

The theoretical framework of ecological resilience suggests that discontinuities in the hierarchy and dynamics of a complex system should generate a heterogeneous landscape (Holling, 1992). The heterogeneity in question is manifested through the existence of discontinuities produced in the texture of the landscape. In this research the heterogeneity of a built environment will be tested as the interrelationship between richness, diversity and evenness.

The resilience theory, through the TDH, explains that discontinuities in the landscape occur because elements of similar sizes tend to be aggregated so as to use resources at a similar scale of time and space. The quantity of land that is 
used for blocks differs from the quantity of land that a house uses. At the same time blocks and building footprints also change at different rates in time (see chapter 4). There is a relationship between the scale of aggregations and sizes of elements (see chapter 5). Self-organization dynamics produce clustering processes of elements of discrete sizes that result in the generation of aggregations and discontinuities (Gunderson et al., 2009).

Aggregations can be found quantitatively by clustering data, like similar areas of blocks or plots that can constitute a group of similar sizes. For example, it is possible to find aggregations of big, medium and small blocks. In this case the structure has three aggregations (big, medium and small blocks) and two discontinuities, one between big and medium sizes and the other one between medium and small blocks sizes. The structure of aggregations and discontinuities, a gaps assemblage, describes the composition of the texture of a landscape that can be understood as the structure of its morphology. Discontinuities in the structure of an urban landscape define the level of difference between clusters. Difference represents an important characteristic that enables diversity. More diverse systems should be linked with more heterogeneous landscapes. A large number and size of discontinuities within an assemblage would imply a richer and more diverse organization that should be reflected in a higher resilience capacity for the system (C. Allen et al., 2005). An urban landscape with high resilience capacity is the product of the richness and diversity of its urban Panarchy. This structure will try to maintain its heterogeneity in a fairly stable way in the face of unpredictable change through time. Accordingly, with this assumption urban landscapes that are more heterogeneous will change less in their structures than urban landscapes with a lesser heterogeneity. Moreover, this would imply that the complexity, heterogeneity and diversity presented in the built environment of an urban landscape are produced from the adaptability provided by its resilience capacity and linked with its discontinuity.

\subsection{Case studies}

The analysis compares changes in the diversity of the structure of two urban landscapes: the neighbourhood of Nezu in Tokyo, Japan and one area of 
the city business district of Auckland, New Zealand. The boundaries of the area of study of the east side of Auckland CBD, used in chapter 4, were adjusted in order to create an area similar to that of Nezu. Both areas represent a mixture between residential and commercial uses, and both were developing through time and showing resilience to the different historical events that could provoke change. However, historical events and processes were shaping these areas in a different manner. Furthermore, the case study area in Auckland has an important public green space and the area in Tokyo has only private green spaces. Both differences and similarities between these two case studies are considered important for comparison. The motive for comparing the dynamics of change in two urban landscapes is to try to find similarities in their behaviours while acknowledging their differences, in other words to find some external validation for the research.

For the purpose of this analysis five historical periods were chosen for both case studies. These periods follow the main historical phases in the evolution of Auckland and Tokyo. The chosen periods for Tokyo are: 1888 to 1919, 1919 to 1932, 1932 to 1956 and 1956 to 2003. Periods in Auckland are: 1882 to 1908,1908 to 1931,1931 to 1966 and 1966 to 2006.

\subsection{Methods}

The overall method is based on use of discontinuities to determine the relative resilience of ecosystems (C. Allen et al., 2005). The analysis was carried out in two urban landscapes, these being Nezu and the traditional centre of the Auckland CBD. The elements of their built environments were mapped and quantified through all periods. The analysis was conducted by measuring two group of variables: elements of the built environment (BF, PL, BL and ST) and green spaces (GS). A resilient assemblage approach (Garcia et al., 2012; Muminovic et al., 2012a) understands green spaces assembled within the built environment. In order to observe the interactions between green spaces and the built environment, green spaces were isolated and compared in order to see how they are tied to resilience dynamics (see section 2.7).

The elements of the built environment were analysed based on their areas measured in $\mathrm{m}^{2}$. Green spaces comprise both public and private areas and 
were measured at the level of blocks, meaning that they were defined as the area of the block minus building footprint areas. Private areas correspond with domestic green spaces (Gaston, Warren, Thompson, \& Smith, 2005). Public green spaces were only found in Auckland and are represented by parks. In accordance with the theoretical assumptions of this research, green public spaces were counted as extensions of every plot, consequently the total area of parks was divided proportionally.

The method has two phases: a qualitative and a quantitative analysis. The objective of the qualitative analysis is the definition of the structure of the urban landscape. and mapping how these aggregations are clustered in space. The objective is to discover and define the number of aggregations (groups of more characteristic sizes) and discontinuities (the distance between aggregations that separate groups from each other) that is present in each variable of the built environment, describing in this way their structures. Change in the size of each variable was measured for the periods set out above. The objective was to measure the quantity of change (change difference) and the speed of change (rate of change) of PL, BL, ST in order to have two dimensions assessed-change in time and change in space. Alteration in the size of PL, BL, ST and green spaces (BF were not considered because the information was incomplete in Nezu) between periods was defined as change. Change was measured as change difference and as rate of change. The latter is the area difference between two successive periods divided by number of years between periods.

The quantitative analysis, focuses on the measurement of change in the different structures that emerged from the first analysis. The aim is to define how heterogeneous each structure is and also in which way it is changing in order to test the resilience capacity of Nezu and Auckland through changes in the heterogeneity of their built environments. Heterogeneity in this research is defined as an average between the values of richness, evenness and diversity related with the set of aggregations and discontinuities discovered in the structure of PL, BL, ST and GS per year. The analysis of discontinuities and aggregations used as a reference the method described by Allen in the assessment of specific resilience in ecosystems but adapted to the measurement of urban landscapes (C. Allen et al., 2005). 


\subsection{Qualitative analysis}

The structure of each element of the urban landscape (BF, PL, BL, ST) was defined as the order of aggregations and discontinuities based on the size of elements. In order to investigate the presence of discontinuities in the urban landscape a cluster analysis was conducted (Garmestani et al., 2005) using Weka software that characterizes and defines groups of variables that cluster around a few points in the database (Witten et al., 2011). The cluster analysis was done using EM (expected maximization) -I 100 -N -1 -M 1.0E-6 -S 100, where max. alterations equal to 100 , min. standard deviation equals to $1.0 \mathrm{E}-6$, the number of cluster equals to -1 , and the seed equals to 100 (see section 3.5). The discontinuities and aggregations obtained were readjusted in relation to their sizes to the maximum size of the cluster at each level. Using this method only significantly large discontinuities and clusters were considered for further analysis. Aggregations and discontinuities in PL, BL, ST and GS obtained from the cluster analysis of the urban landscape of Nezu and Auckland in each year were illustrated chronologically using bars and gaps.

Graphics were organized as an assemblage of horizontal bars (aggregations) and gaps between bars (discontinuities). The size of each bar is the range between the smallest and the biggest element contained in that aggregation. Gaps are distances between the biggest element of an aggregation and the smallest element of the next aggregation. The degree of grey inside bars characterizes the quantity of elements (BL, PL or ST) included in each aggregation. The information obtained in Weka was exported to excel tables and then these were joined to the database contained in the maps drawn in QGIS (see appendices 11 to 21). A first set of observations was carried out by contrasting results about alterations in the constitution of the structure of green spaces and built environments in Nezu and Auckland. Alterations in the structure of each case study were graphically observed through a set of criteria: number of clusters (richness), the size of each aggregation (range), the number of entities in each cluster (distribution), and the size of gaps between aggregations (difference). 

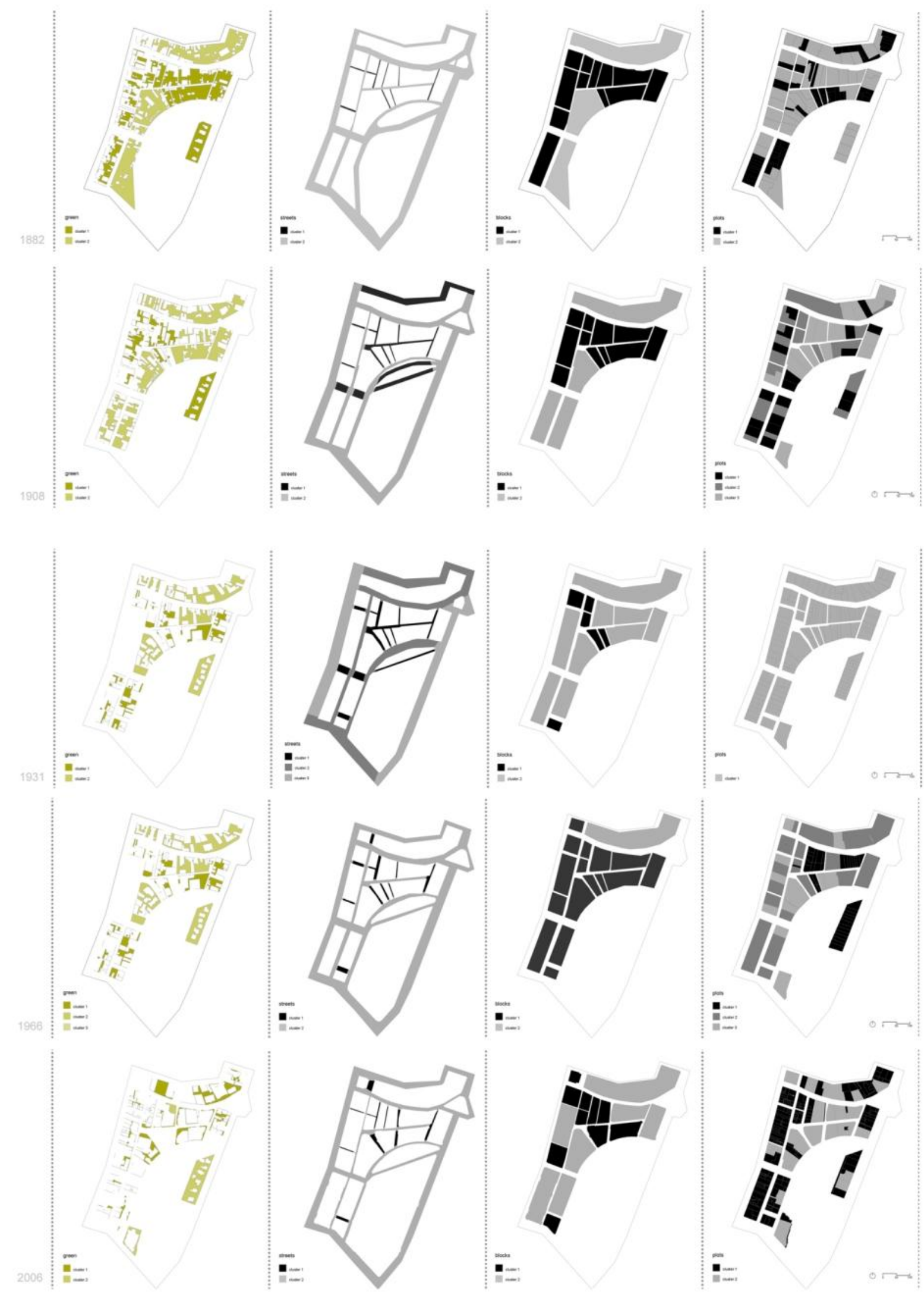

Figure 6-1. The evolution of heterogeneity in the urban landscape of Auckland 


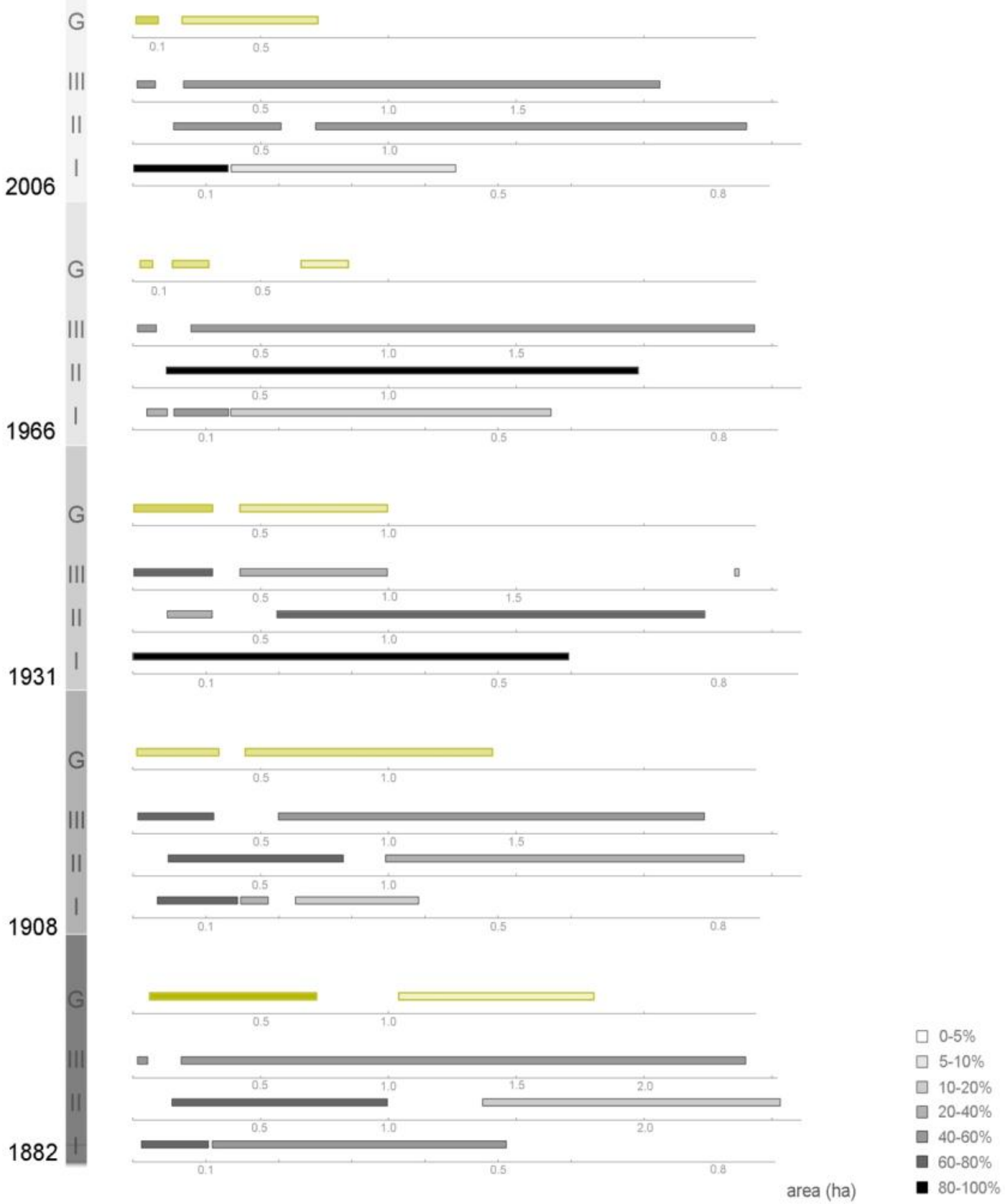

Figure 6-2. Structures of aggregations and discontinuities in GS, ST, BL, PL in Auckland 


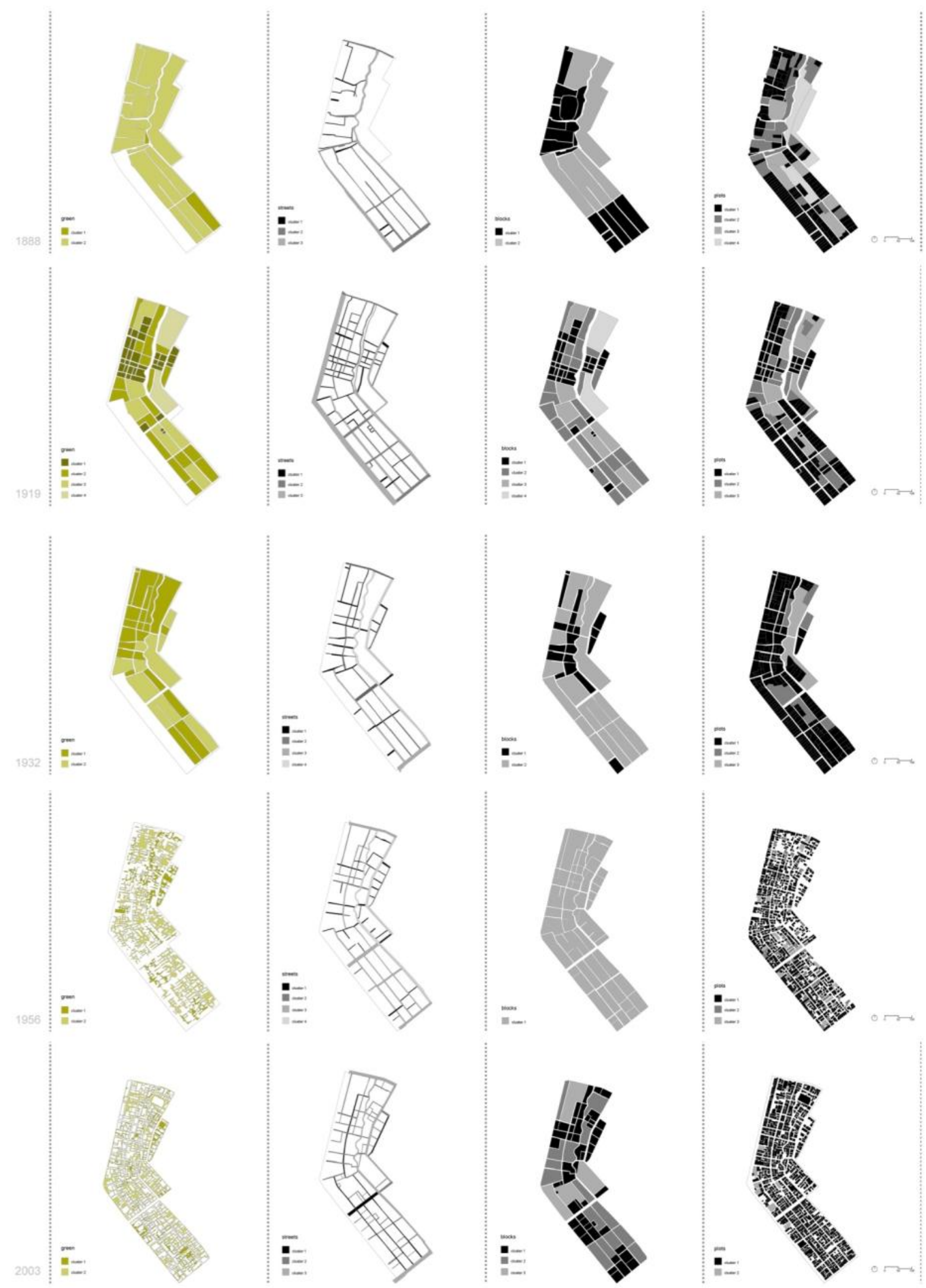

Figure 6-3. The evolution of heterogeneity in the urban landscape of Nezu 


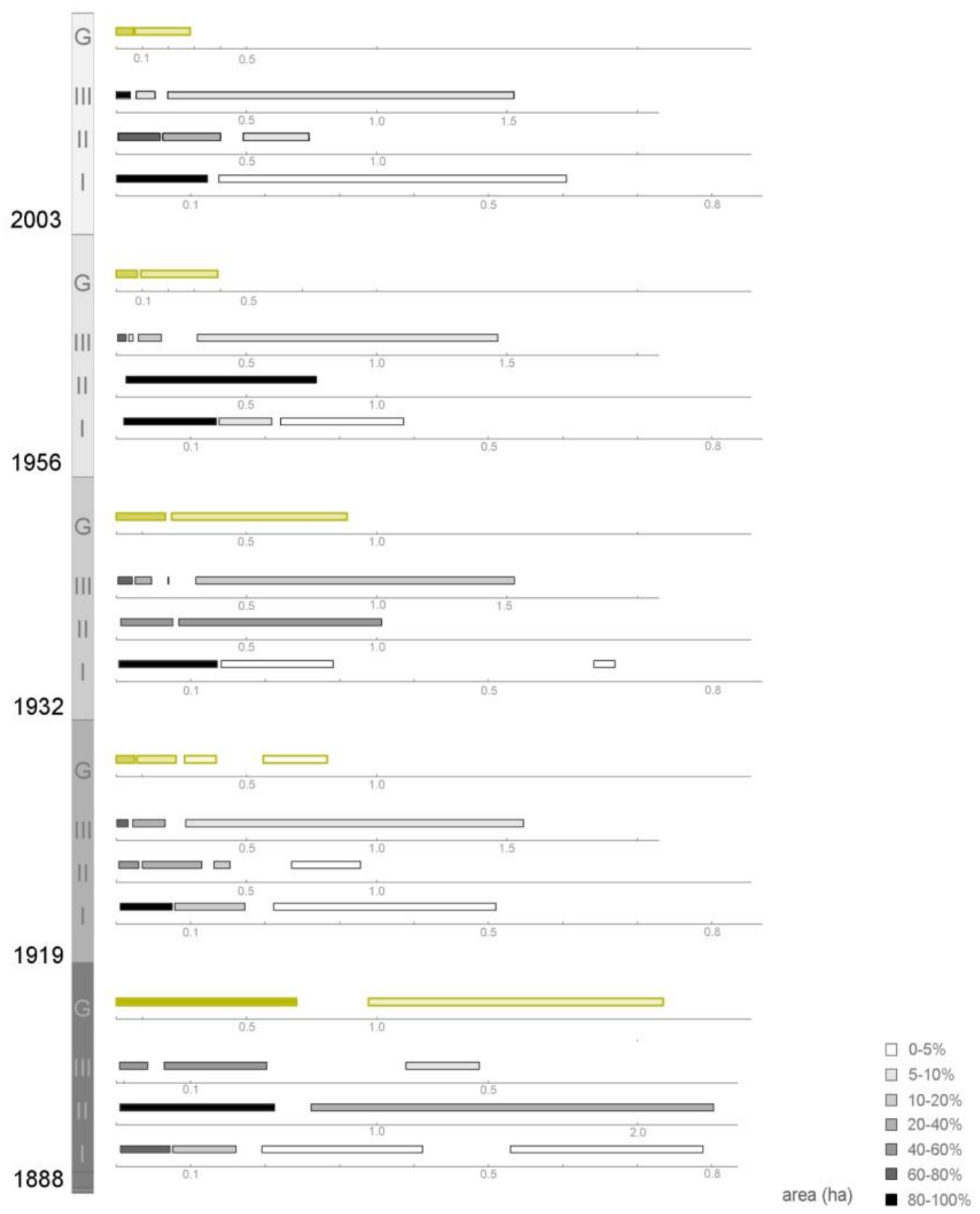

Figure 6-4. Structures of aggregations and discontinuities in GS, ST, BL, PL in Nezu 
Table 6-1. Comparison between diversity and rate of change in GS and BE of Auckland and Nezu

\begin{tabular}{|c|c|c|}
\hline Nezu & Space (diversity) & Time (rate of change) \\
\hline $\begin{array}{l}\text { Green Spaces } \\
\text { (GS) }\end{array}$ & $\begin{array}{l}\text {-Sizes of green spaces are decreasing from } \\
1888 \text { to } 2003 . \\
\text {-The largest number of elements is always } \\
\text { clustered in the aggregations of smallest size } \\
\text { elements. } \\
\text {-In } 1919 \text { the structure has the biggest number } \\
\text { of clusters and average range of them is } \\
\text { smaller than } 1888 \text {. } \\
\text {-The relationship between the range and the } \\
\text { distribution of aggregations maintains the } \\
\text { same proportions from } 1932 \text { to } 2003 \text {. }\end{array}$ & $\begin{array}{l}\text { - Three phases. From } 1888 \text { to } \\
1932 \text {, slow change; from } 1932 \\
\text { to } 1956 \text { fast change; from } \\
1956 \text { to } 2003 \text { slow change. } \\
\text { - The rate of change in the } \\
\text { richness of aggregations also } \\
\text { has two phases: from } 1888 \text { to } \\
1932 \text { fast change and from } \\
1932 \text { to } 2003 \text { slow change. }\end{array}$ \\
\hline $\begin{array}{l}\text { Built } \\
\text { environment } \\
\text { (BE) }\end{array}$ & $\begin{array}{l}\text { - The structure of the streets remain fairly } \\
\text { stable across the years } \\
\text { - The structure was getting less rich from } 1919 \\
\text { to } 2003 \text {. } \\
\text { - Changes in plots and blocks caused the major } \\
\text { disturbances in the structure of the BE. } \\
\text { - From } 1888 \text { to } 1956 \text { the diversity in the range } \\
\text { of aggregations in blocks is clustering around } \\
\text { one size. The distance between aggregations is } \\
\text { decreasing. } \\
\text { - Plots exhibit changes in their distance and } \\
\text { richness from } 1888 \text { to } 2003 \text {. }\end{array}$ & $\begin{array}{l}\text {-All the levels of the structure } \\
\text { followed the same trend with } \\
\text { a breaking point in } 1932 \text {. } \\
\text {-There are two phases at all } \\
\text { levels: a phase of fast change } \\
\text { from } 1888 \text { to } 1932 \text { and a long } \\
\text { phase of slow change till } \\
2003 \text {. }\end{array}$ \\
\hline GS+BE & $\begin{array}{l}\text { Diversity of GS and BE experience similar } \\
\text { changes from } 1888 \text { to } 1956 \text {. However from } \\
1956 \text { to } 2003 \mathrm{GS} \text { are clearly constant while the } \\
\text { structure of BE experienced changes in its } \\
\text { richness and range without changing its } \\
\text { diversity. }\end{array}$ & $\begin{array}{l}\text { Their rates of change show } \\
\text { one cycle in the case of } \mathrm{BE} \\
\text { and more than one in GS. } \\
\text { Phases in GS and BE have } \\
\text { different qualities. GS show } \\
\text { less amplitude of change than } \\
\text { BE. }\end{array}$ \\
\hline \multicolumn{3}{|l|}{ Auckland } \\
\hline $\begin{array}{l}\text { Green Spaces } \\
\text { (GS) }\end{array}$ & $\begin{array}{l}\text { - GS maintain their richness with the exception } \\
\text { of } 1966 . \\
\text { - The range of aggregations is decreasing from } \\
1882 \text { to } 2006 . \\
\text { - Distribution within aggregations is } \\
\text { concentrated in small elements in } 1882,1932 \\
\text { and } 2006 \text {. In } 1908 \text { and } 1966 \text { the distribution is } \\
\text { more even. } \\
\text { - In } 1966 \text { the pattern of one small aggregation } \\
\text { and one bigger aggregation is disrupted into } \\
\text { three small aggregations. } \\
\text { - In } 1966 \text { the structure shows the biggest gap } \\
\text { in its evolution. }\end{array}$ & $\begin{array}{l}\text { There are three phases. The } \\
\text { first one shows a constancy in } \\
\text { its rate of change, from } 1882 \\
\text { to } 1931 \text {; the second phase } \\
\text { from } 1931 \text { to } 1966 \text { shows } \\
\text { slow change and the third } \\
\text { phase, from } 1966 \text { to } 2003 \text {, } \\
\text { exhibits fast change. }\end{array}$ \\
\hline $\begin{array}{l}\text { Built } \\
\text { environment } \\
\text { (BE) }\end{array}$ & $\begin{array}{l}\text { - The range of aggregations does not generally } \\
\text { experience big change with the exception of } \\
1908 \text { when the structure is richer. } \\
\text { - Blocks showed a decrease in richness in } 1966 \\
\text { and plots in } 1931\end{array}$ & $\begin{array}{l}\text { Blocks, plots and streets show } \\
\text { different phases. Streets have } \\
\text { one constant phase, blocks } \\
\text { two phases, slow change from } \\
1882 \text { to } 1931 \text { and fast change }\end{array}$ \\
\hline
\end{tabular}




\begin{tabular}{|l|l|l|}
\hline & $\begin{array}{l}\text { - From 1882 to 1931 the structure of BE } \\
\text { experienced change in the distance between } \\
\text { aggregations. } \\
\text { The position of the gaps is moving across the } \\
\text { structure. }\end{array}$ & $\begin{array}{l}\text { until 2006. Plots show three } \\
\text { phases. }\end{array}$ \\
\hline GS+BE & $\begin{array}{l}\text { - When green spaces are changing BE is not } \\
\text { changing in the same way. From 1882 to 1931 }\end{array}$ & $\begin{array}{l}\text { While GS show slow change } \\
\text { the BE exhibits fast change in } \\
\text { GS keep the distribution and position of gaps } \\
\text { blocks and plots. When fast } \\
\text { chile the same variables behave in the occurs in GS blocks } \\
\text { opposite way in BE. From 1931 to 2006 } \\
\text { relationships are reversed, while GS is } \\
\text { changing BE is not. }\end{array}$ \\
\hline
\end{tabular}

Nezu

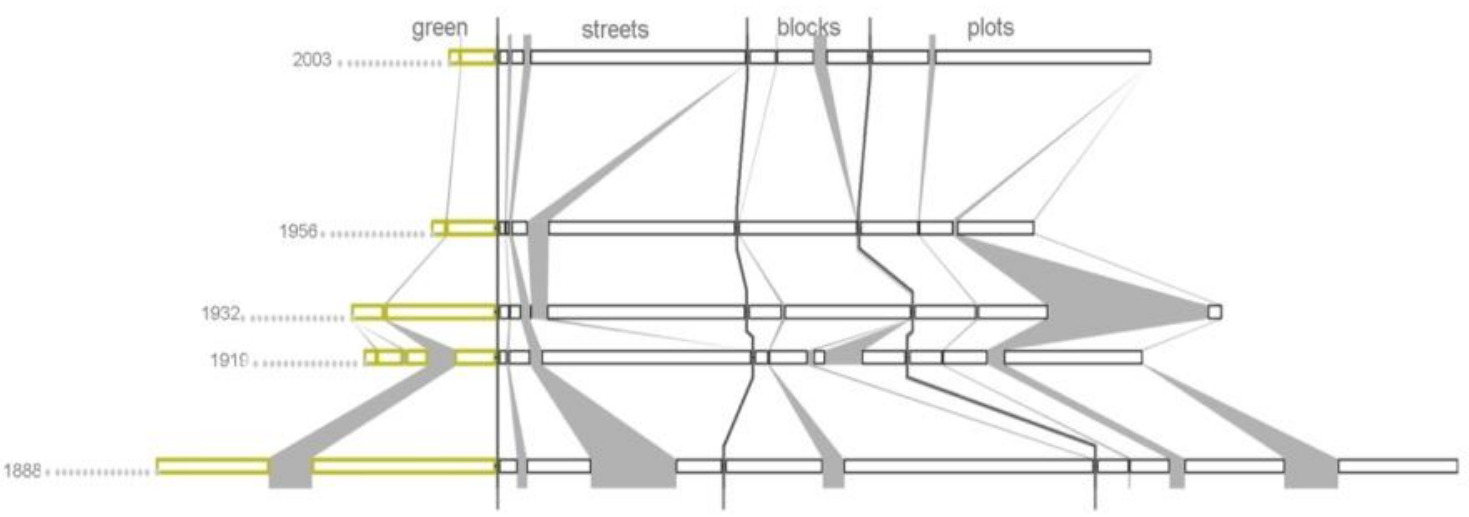

Auckland

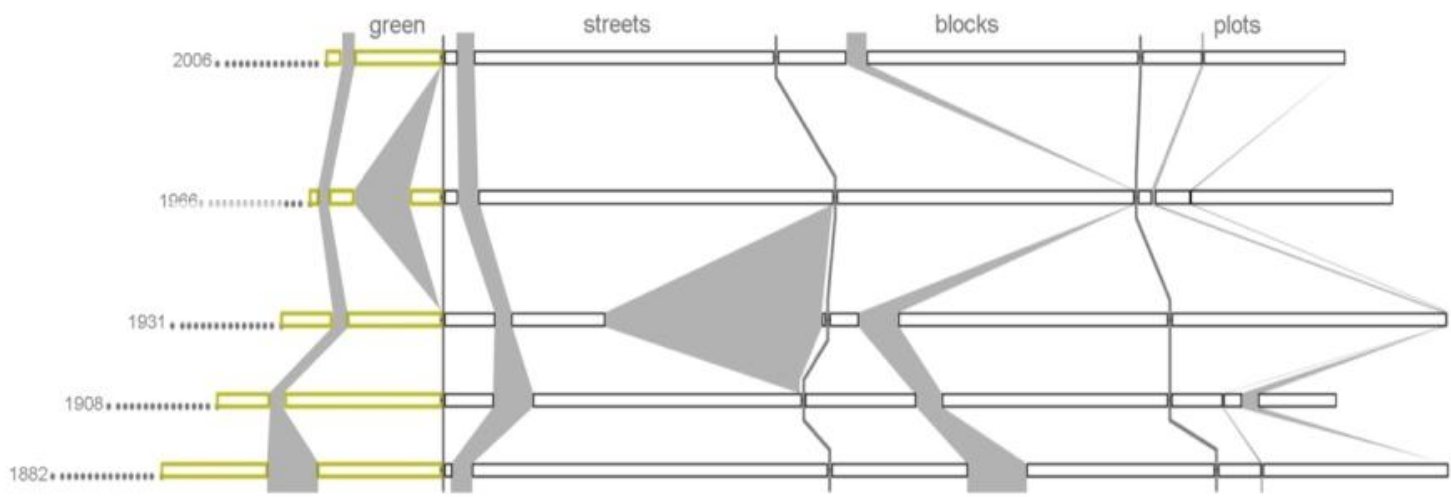

clusters-built environment

clusters-green spaces

discontinuities

Figure 6-5.Comparison between the structure of aggregations and discontinuities in the GS and BE (ST, BL, PL) of Auckland and Nezu 
Table 6-2. Similarities and differences in the behaviour of GS and BE in Auckland and Nezu

\begin{tabular}{|c|c|c|}
\hline & Similarities & Differences \\
\hline $\begin{array}{l}\text { Nezu and } \\
\text { Auckland }\end{array}$ & $\begin{array}{l}\text { - GS contribute to diversity of Nezu and } \\
\text { Auckland. } \\
\text { - Periods with one aggregation are not } \\
\text { repeated. } \\
\text { - GS always exhibit two clusters. } \\
\text { - Range of GS in their first periods is evenly } \\
\text { distributed and the biggest. } \\
\text {-An increase on the richness of GS only } \\
\text { happened once in each structure. } \\
\text { - Structural changes at any level are neither } \\
\text { linear nor continuous. } \\
\text { - Structural changes are more frequent within } \\
\text { levels than in the whole structure. The rate of } \\
\text { change in GS does not follow the rate of } \\
\text { change of BE }\end{array}$ & $\begin{array}{l}\text { - Rate of change in GS in Nezu is } \\
\text { smooth while in Auckland it is } \\
\text { not. } \\
\text { - In Nezu change in GS mirrored } \\
\text { change in BE. In Auckland change } \\
\text { in GS behaves in the opposite way } \\
\text { to BE. } \\
\text {-Number of discontinuities in } \\
\text { Nezu is bigger that in Auckland. } \\
\text {-ST, BL, PL in Auckland are less } \\
\text { rich than in Nezu. } \\
\text {-The range of aggregations in } \\
\text { Nezu is getting smaller while in } \\
\text { Auckland it remains constant. }\end{array}$ \\
\hline
\end{tabular}

\subsection{Quantitative analysis}

The qualitative analysis illustrated in figures 6-4 and 6-5 provides information about the way in which the structure of the urban landscape of Nezu and Auckland has been changing. It gives information visually but does not provide the numbers for a comparative analysis.

BL, PL, ST, GS were considered as different levels in the urban landscapes. Instead of making an analysis of the number of functional groups within each level (PL, BL, ST, GS) the richness of the systems was defined in relationship with the number of aggregations per level. For example, in figure 6-5 it is possible to observed that streets in 2003 in Nezu have three bars while Auckland in 2008 has two bars. These bars correspond with the quantity of groups of similar sizes in each data base. In this research richness was linked with the number of groups of similar sizes (aggregations) within a variable (PL, ST, BL, BF). A major quantity of aggregations in the same level implies more groups of different sizes, therefore a more diverse structure. Consequently in the comparison of the structure of streets between Nezu and Auckland, streets in Nezu are richer in the quantity of aggregations than in Auckland. The full list with the assessment of richness in elements of the urban landscape, at all periods, for Auckland and Nezu, is analysed and presented in tables 6-3 and 6-4.

Diversity was calculated using the Shannon-Wiener diversity index $H^{\mathbf{\prime}}=-\sum p i \ln p i$ where $p i=1 / \log S(\mathrm{~S}=$ total number of elements). Evenness was 
calculated using the formula suggested by Pielou $J=H^{\prime} / H^{\prime} \max =H^{\prime} / \log S$ where $H^{\prime}$ is the Shannon-Wiener diversity. In order to be able to compare the performance of Auckland and Nezu period by period, values of richness, evenness and diversity in plots, blocks, streets and green spaces were averaged. The average performance is presented in table 6-5.

In order to make observations of the evolution of the heterogeneity in Nezu and Auckland a similar approach to the qualitative analysis was followed: assessment of change in quantity (space) and speed (time). Change in the quantity of heterogeneity was analysed as change difference between consecutive periods. Change in the speed of change was implemented by using rate of change in the heterogeneity between periods analysed.

\subsubsection{Analysis of heterogeneity within scale}

The qualitative analysis permits having a quick graphic panorama that illustrates the state of the system, however quantitative appreciations done with this method demand an important investment of time without the certainty of arriving at precise conclusions. These considerations are not related with the quantity of GS but with their evenness, diversity and richness.

Table 6-3. Analysis of the evolution of richness, evenness and diversity in Auckland

\begin{tabular}{|l|l|l|l|l|l|l|l|l|}
\hline years & Auckland BE & A1 & A2 & A3 & Elements & Richness & Evenness & Diversity \\
\hline 2006 & Plots & 179 & 20 & & 199 & 2 & 0.4706 & 0.3262 \\
\hline 2006 & Blocks & 7 & 10 & & 17 & 2 & 0.9774 & 0.6775 \\
\hline 2006 & Streets & 9 & 10 & & 19 & 2 & 0.9980 & 0.6918 \\
\hline 2006 & Green Spaces & 15 & 4 & & 19 & 2 & 0.7425 & 0.5147 \\
\hline 1966 & Plots & 37 & 64 & 10 & 111 & 3 & 0.8197 & 0.9005 \\
\hline 1966 & Blocks & 17 & & & 17 & 1 & 0.0000 & 0.0000 \\
\hline 1966 & Streets & 10 & 10 & & 20 & 2 & 1.0000 & 0.6931 \\
\hline 1966 & Green Spaces & 8 & 8 & 2 & 18 & 3 & 0.8783 & 0.9650 \\
\hline 1931 & Plots & 119 & & & 119 & 1 & 0.0000 & 0.0000 \\
\hline 1931 & Blocks & 6 & 9 & & 15 & 2 & 0.9710 & 0.6730 \\
\hline 1931 & Streets & 11 & 5 & & 16 & 2 & 0.8960 & 0.6211 \\
\hline 1931 & Green Spaces & 12 & 4 & & 16 & 2 & 0.8113 & 0.5623 \\
\hline 1908 & Plots & 50 & 18 & 15 & 83 & 3 & 0.8611 & 0.9460 \\
\hline 1908 & Blocks & 11 & 4 & & 15 & 2 & 0.8366 & 0.5799 \\
\hline 1908 & Streets & 12 & 7 & & 19 & 2 & 0.9495 & 0.6581 \\
\hline 1908 & Green Spaces & 9 & 7 & & 16 & 2 & 0.9887 & 0.6853 \\
\hline
\end{tabular}




\begin{tabular}{|l|l|l|l|l|l|l|l|l|}
1882 & Plots & 64 & 43 & 107 & 2 & 0.9720 & 0.6738 \\
\hline 1882 & Blocks & 12 & 3 & 15 & 2 & 0.7219 & 0.5004 \\
\hline 1882 & Streets & 8 & 10 & 18 & 2 & 0.9911 & 0.6870 \\
\hline 1882 & Green Spaces & 13 & 3 & & 16 & 2 & 0.6962 & 0.4826 \\
\hline
\end{tabular}

Table 6-4. Analysis of the evolution of richness, evenness and diversity in Nezu

\begin{tabular}{|l|l|l|l|l|l|l|l|l|l|}
\hline Years & Nezu BE & A1 & A2 & A3 & A4 & Elements & Richness & Evenness & Diversity \\
\hline 2003 & Plots & 1165 & 15 & & & 1180 & 2 & 0.0983 & 0.0681 \\
\hline 2003 & Blocks & 51 & 14 & 5 & & 70 & 3 & 0.6746 & 0.7411 \\
\hline 2003 & Streets & 53 & 6 & 6 & & 65 & 3 & 0.5519 & 0.6063 \\
\hline 2003 & Green Spaces & 55 & 16 & & & 71 & 2 & 0.7698 & 0.5336 \\
\hline 1956 & Plots & 880 & 52 & 14 & & 946 & 3 & 0.2631 & 0.2891 \\
\hline 1956 & Blocks & 51 & & & & 51 & 1 & 0.0000 & 0.0000 \\
\hline 1956 & Streets & 31 & 4 & 8 & 3 & 46 & 4 & 0.6929 & 0.9606 \\
\hline 1956 & Green Spaces & 59 & 17 & & & 76 & 2 & 0.7668 & 0.5315 \\
\hline 1932 & Plots & 217 & 7 & & & 224 & 2 & 0.2006 & 0.1391 \\
\hline 1932 & Blocks & 18 & 17 & & & 35 & 2 & 0.9994 & 0.6927 \\
\hline 1932 & Streets & 18 & 6 & 2 & 3 & 29 & 4 & 0.7510 & 1.0411 \\
\hline 1932 & Green Spaces & 24 & 11 & & & 35 & 2 & 0.8981 & 0.6225 \\
\hline 1919 & Plots & 163 & 25 & 7 & & 195 & 3 & 0.4848 & 0.5326 \\
\hline 1919 & Blocks & 35 & 24 & 7 & 2 & 68 & 4 & 0.7554 & 1.0472 \\
\hline 1919 & Streets & 36 & 10 & 4 & & 50 & 3 & 0.6922 & 0.7605 \\
\hline 1919 & Green Spaces & 42 & 18 & 6 & 2 & 68 & 4 & 0.6978 & 0.9674 \\
\hline 1888 & Plots & 138 & 27 & 6 & 3 & 174 & 4 & 0.4754 & 0.6591 \\
\hline 1888 & Blocks & 19 & 9 & & & 28 & 2 & 0.9059 & 0.6279 \\
\hline 1888 & Streets & 13 & 8 & 2 & & 23 & 3 & 0.8212 & 0.9022 \\
\hline 1888 & Green Spaces & 21 & 3 & & & 24 & 2 & 0.5436 & 0.3768 \\
\hline
\end{tabular}

Table 6-5. Average performance in heterogeneity in GS, ST, PL, BL of Auckland and Nezu

\begin{tabular}{|l|l|l|l|l|l|l|l|l|l|}
\hline & \multicolumn{9}{|l|}{ Auckland } \\
& Richness & Evenness & Diversity & Mean & & Richness & Evenness & Diversity & Mean \\
\hline GS & 2.200 & 0.823 & 0.642 & 1.222 & GS & 3.400 & 0.702 & 0.854 & 1.652 \\
\hline ST & 2.000 & 0.967 & 0.670 & 1.212 & ST & 2.400 & 0.735 & 0.606 & 1.247 \\
\hline PL & 2.200 & 0.625 & 0.569 & 1.131 & PL & 2.400 & 0.667 & 0.622 & 1.230 \\
\hline BL & 1.800 & 0.701 & 0.486 & 0.996 & BL & 2.800 & 0.304 & 0.338 & 1.147 \\
\hline
\end{tabular}

Nezu has a more heterogeneous built environment (BE) than Auckland. PL, BL, ST and GS in Nezu are richer and more diverse than the same elements in Auckland. Heterogeneity is represented in this case as the mean values that 
resulted from averaging calculations of richness, diversity and evenness in ST, GS, BL and PL at different periods.

The number of aggregations is linked with the number of different groups of sizes in a category or level. For this reason the quantity of aggregations in one structure can be related with its richness. Richer structures are more diverse than even structures; therefore, richness represents a key variable for determining the heterogeneity of the landscape.

In Auckland, GS is the most heterogeneous element of the BE and ST is the most diverse. PL has an equally important richness but its values change critically between periods. In Nezu, ST is the most heterogeneous and richest element of the BE followed by GS. In both cases ST and GS play an important role in the heterogeneity of the BE. One common denominator between ST and GS is that neither of these are elements of the urban landscape that contain areas with building footprints. ST and GS represent non-built spaces. The major diversity and richness of GS and ST observed in Nezu and Auckland highlights the importance of intervals in between built spaces as producers of heterogeneity in the structure of the BE. In this way GS and ST add to the complexity and resilience of the system.

\subsubsection{Analysis of heterogeneity across scales}

The purpose of this part of the analysis is to test how the heterogeneity of the BE and GS are related. Particular attention is paid to the analysis of change in the heterogeneity of BE and GS. The urban Panarchy of Nezu and Auckland should tend to keep both structures stable across time even though some of their elements are lost or changed. Following the theory developed in ecological resilience, more heterogeneous structures should exhibit a higher resilience capacity. Therefore, the urban landscape of Nezu should have a higher resilience capacity and should change less in its structure than the structure of the urban landscape of Auckland.

The analysis uses variables of richness, evenness, diversity and their average in order to characterize the heterogeneity of the elements of the urban landscape of Nezu and Auckland across the scales of BE and GS and also through different periods of time. The structures of the urban landscapes of Auckland and 
Nezu are compared by contrasting changes in the heterogeneity of their built environments with and without green spaces. As a result three parallel analyses are conducted in order to observe the performances of Nezu and Auckland.

\subsubsection{Evolution of the heterogeneity of Nezu and Auckland GS and BE}

Table 6-6. Heterogeneity of Nezu and Auckland BE and GS

\begin{tabular}{|l|l|l|l|l|l|l|l|l|l|}
\hline \multicolumn{4}{|l|}{ Nezu Built environment +Green Spaces } & \multicolumn{4}{l|}{ Auckland Built environment +Green Spaces } \\
\hline & Richness & Evenness & Diversity & Mean & & Richness & Evenness & Diversity & Mean \\
\hline 2003 & 2.5000 & 0.5236 & 0.4873 & 1.1703 & 2006 & 2.0000 & 0.7971 & 0.5525 & 1.1165 \\
\hline 1956 & 2.5000 & 0.4307 & 0.4453 & 1.1253 & 1966 & 2.2500 & 0.6745 & 0.6397 & 1.1881 \\
\hline 1932 & 2.5000 & 0.7123 & 0.6238 & 1.2787 & 1931 & 1.7500 & 0.6696 & 0.4641 & 0.9612 \\
\hline 1919 & 3.5000 & 0.6576 & 0.8269 & 1.6615 & 1908 & 2.2500 & 0.9090 & 0.7173 & 1.2921 \\
\hline 1888 & 2.7500 & 0.6865 & 0.6415 & 1.3593 & 1882 & 2.0000 & 0.8453 & 0.5859 & 1.1437 \\
\hline Mean & 2.7500 & 0.6021 & 0.6050 & 1.3190 & Mean & 2.0500 & 0.7791 & 0.5919 & 1.1403 \\
\hline stdev & 0.4330 & 0.1203 & 0.1502 & 0.2122 & stdev & 0.2092 & 0.1055 & 0.0948 & 0.1204 \\
\hline var & 0.1875 & 0.0145 & 0.0226 & 0.0450 & var & 0.0438 & 0.0111 & 0.0090 & 0.0145 \\
\hline
\end{tabular}

Table 6-7. Heterogeneity of Nezu BE and Auckland BE

\begin{tabular}{|l|l|l|l|l|l|l|l|l|l|l|}
\hline \multicolumn{4}{|l|}{ Nezu Built environment } & \multicolumn{4}{l|}{ Auckland Built environment } \\
\hline & Richness & Evenness & Diversity & Mean & & Richness & Evenness & Diversity & Mean \\
\hline 2003 & 2.6667 & 0.4416 & 0.4718 & 1.1934 & 2006 & 2.0000 & 0.8153 & 0.5651 & 1.1268 \\
\hline 1956 & 2.6667 & 0.3187 & 0.4166 & 1.1340 & 1966 & 2.0000 & 0.6066 & 0.5312 & 1.0459 \\
\hline 1932 & 2.6667 & 0.6503 & 0.6243 & 1.3138 & 1931 & 1.6667 & 0.6223 & 0.4314 & 0.9068 \\
\hline 1919 & 3.3333 & 0.6441 & 0.7801 & 1.5859 & 1908 & 2.3333 & 0.8824 & 0.7280 & 1.3146 \\
\hline 1888 & 3.0000 & 0.7342 & 0.7297 & 1.4880 & 1882 & 2.0000 & 0.8950 & 0.6204 & 1.1718 \\
\hline Mean & 2.8667 & 0.5578 & 0.6045 & 1.3430 & Mean & 2.0000 & 0.7643 & 0.5752 & 1.1132 \\
\hline stdev & 0.2981 & 0.1716 & 0.1580 & 0.1917 & stdev & 0.2357 & 0.1402 & 0.1097 & 0.1511 \\
\hline var & 0.0889 & 0.0295 & 0.0250 & 0.0367 & var & 0.0556 & 0.0197 & 0.0120 & 0.0228 \\
\hline
\end{tabular}

Table 6-8. Heterogeneity of Nezu GS and Auckland GS

\begin{tabular}{|l|l|l|l|l|l|l|l|l|l|}
\hline \multicolumn{9}{|l|}{ Nezu Green Spaces } & \multicolumn{4}{l|}{ Auckland Green Spaces } \\
\hline & Richness & Evenness & Diversity & Mean & & Richness & Evenness & Diversity & Mean \\
\hline 2003 & 2.0000 & 0.7698 & 0.5336 & 1.1011 & 2006 & 2.0000 & 0.7425 & 0.5147 & 1.0857 \\
\hline 1956 & 2.0000 & 0.7668 & 0.5315 & 1.0995 & 1966 & 3.0000 & 0.8783 & 0.9650 & 1.6144 \\
\hline 1932 & 2.0000 & 0.8981 & 0.6225 & 1.1735 & 1931 & 2.0000 & 0.8113 & 0.5623 & 1.1245 \\
\hline 1919 & 4.0000 & 0.6978 & 0.9674 & 1.8884 & 1908 & 2.0000 & 0.9887 & 0.6853 & 1.2247 \\
\hline 1888 & 2.0000 & 0.5436 & 0.3768 & 0.9734 & 1882 & 2.0000 & 0.6962 & 0.4826 & 1.0596 \\
\hline Mean & 2.4000 & 0.7352 & 0.6064 & 1.2472 & Mean & 2.2000 & 0.8234 & 0.6420 & 1.2218 \\
\hline stdev & 0.8944 & 0.1293 & 0.2203 & 0.3656 & stdev & 0.4472 & 0.1153 & 0.1963 & 0.2283 \\
\hline var & 0.8000 & 0.0167 & 0.0485 & 0.1337 & var & 0.2000 & 0.0133 & 0.0385 & 0.0521 \\
\hline
\end{tabular}


The comparative analysis shows that the BE of Nezu is more heterogeneous than the BE of Auckland with and without GS. In GS in both Auckland and Nezu, heterogeneity is quite similar with the difference that richness in Nezu is higher. However the contribution of Auckland GS to the heterogeneity of its urban landscape is more important than in the case of Nezu GS. This situation can be explained because Auckland GS richness and diversity is bigger than the heterogeneity of its BE, therefore it can lift its value.

In Nezu and Auckland, GS contribute to the complexity and heterogeneity of the BE but the level of importance of the contribution is related with the diversity and richness of their BE. In consequence the heterogeneity of a BE will define the level of effectiveness of the contribution GSs can make in an urban landscape. A BE that has a structure of PL, ST and BL that is not rich and diverse enough would need a highly heterogeneous system of GS to become a richer urban landscape.

\subsubsection{Difference in change in the heterogeneity of Nezu and Auckland with and without Green Space}

Table 6-9. Change difference in Nezu and Auckland BE and GS

\begin{tabular}{|c|c|c|c|c|c|c|c|c|c|}
\hline \multicolumn{5}{|c|}{ Nezu Change Difference BE+GS } & \multicolumn{5}{|c|}{ Auckland Change Difference BE+GS } \\
\hline Period & Richness & Evenness & Diversity & Mean & Periods & Richness & Evenness & Diversity & Mean \\
\hline $\begin{array}{l}1956- \\
2003 \\
\end{array}$ & 0.0000 & 0.1774 & 0.0861 & 0.0879 & $\begin{array}{l}1996- \\
2006\end{array}$ & 0.1111 & 0.1538 & 0.1362 & 0.1337 \\
\hline $\begin{array}{l}1932- \\
1956 \\
\end{array}$ & 0.0000 & 0.3953 & 0.2862 & 0.2272 & $\begin{array}{l}1931- \\
2006\end{array}$ & 0.2222 & 0.0073 & 0.2744 & 0.1680 \\
\hline $\begin{array}{l}1919- \\
1932 \\
\end{array}$ & 0.2857 & 0.0768 & 0.2456 & 0.2027 & $\begin{array}{l}1908- \\
1931 \\
\end{array}$ & 0.2222 & 0.2634 & 0.3530 & 0.2795 \\
\hline $\begin{array}{l}1888- \\
1919 \\
\end{array}$ & 0.2143 & 0.0422 & 0.2242 & 0.1602 & $\begin{array}{l}1882- \\
1908 \\
\end{array}$ & 0.1111 & 0.0700 & 0.1832 & 0.1214 \\
\hline Mean & 0.1250 & 0.1729 & 0.2105 & 0.1695 & Mean & 0.1667 & 0.1236 & 0.2367 & 0.1757 \\
\hline stdev & 0.1473 & 0.1589 & 0.0868 & 0.0610 & stdev & 0.0642 & 0.1108 & 0.0965 & 0.0905 \\
\hline var & 0.0217 & 0.0253 & 0.0075 & 0.0037 & var & 0.0041 & 0.0123 & 0.0093 & 0.0052 \\
\hline
\end{tabular}

Table 6-10. Change difference in Nezu and Auckland BE

\begin{tabular}{|l|l|l|l|l|l|l|l|l|l|}
\hline \multicolumn{4}{|l|}{ Nezu Change Difference BE } & \multicolumn{4}{l|}{ Auckland Change Difference BE } \\
\hline $\begin{array}{l}\text { Period } \\
\text { 1956- }\end{array}$ & Richness & Evenness & Diversity & Mean & Periods & Richness & Evenness & Diversity & Mean \\
\hline 2003 & 0.0000 & 0.2783 & 0.1172 & 0.1318 & $\begin{array}{l}1996- \\
2006\end{array}$ & 0.0000 & 0.2561 & 0.0600 & 0.1054 \\
\hline $1932-$ & 0.0000 & 0.5100 & 0.3328 & 0.2809 & $1931-$ & 0.1667 & 0.0253 & 0.1880 & 0.1267 \\
\hline
\end{tabular}




\begin{tabular}{|l|l|l|l|l|l|l|l|l|l|}
1956 & & & & & 2006 & & & & \\
\hline $\begin{array}{l}1919- \\
1932\end{array}$ & 0.2000 & 0.0096 & 0.1997 & 0.1364 & $\begin{array}{l}1908- \\
1931\end{array}$ & 0.2857 & 0.2947 & 0.4075 & 0.3293 \\
\hline $\begin{array}{l}1888- \\
1919\end{array}$ & 0.1000 & 0.1227 & 0.0645 & 0.0957 & $\begin{array}{l}1882- \\
1908\end{array}$ & 0.1429 & 0.0141 & 0.1478 & 0.1016 \\
\hline Mean & 0.0750 & 0.2301 & 0.1785 & 0.1612 & Mean & 0.1488 & 0.1476 & 0.2008 & 0.1657 \\
\hline stdev & 0.0957 & 0.2167 & 0.1169 & 0.0818 & stdev & 0.1172 & 0.1485 & 0.1478 & 0.1378 \\
\hline var & 0.0092 & 0.0469 & 0.0137 & 0.0067 & var & 0.0137 & 0.0221 & 0.0218 & 0.0120 \\
\hline
\end{tabular}

Table 6-11. Change difference in Nezu and Auckland GS

\begin{tabular}{|l|l|l|l|l|l|l|l|l|l|}
\hline \multicolumn{9}{|l|}{ Nezu Change Difference GS } \\
\hline $\begin{array}{l}\text { Period } \\
\text { Richness }\end{array}$ & Evenness & Diversity & Mean & Period & Richness & Evenness & Diversity & Mean \\
\hline $\begin{array}{l}1956- \\
2003\end{array}$ & 0.0000 & 0.0039 & 0.0039 & 0.0026 & $\begin{array}{l}1996- \\
2006\end{array}$ & 0.3333 & 0.1547 & 0.4667 & 0.3182 \\
\hline $\begin{array}{l}1932- \\
1956\end{array}$ & 0.0000 & 0.1461 & 0.1461 & 0.0974 & $\begin{array}{l}1931- \\
2006\end{array}$ & 0.3333 & 0.0764 & 0.4172 & 0.2756 \\
\hline $\begin{array}{l}1919- \\
1932\end{array}$ & 0.5000 & 0.2230 & 0.3565 & 0.3598 & $\begin{array}{l}1908- \\
1931\end{array}$ & 0.0000 & 0.1794 & 0.1794 & 0.1196 \\
\hline $\begin{array}{l}1888- \\
1919\end{array}$ & 0.5000 & 0.2210 & 0.6105 & 0.4439 & $\begin{array}{l}1882- \\
1908\end{array}$ & 0.0000 & 0.2958 & 0.2958 & 0.1972 \\
\hline Mean & 0.2500 & 0.1485 & 0.2793 & 0.2259 & Mean & 0.1667 & 0.1766 & 0.3398 & 0.2277 \\
\hline stdev & 0.2887 & 0.1028 & 0.2641 & 0.2096 & stdev & 0.1925 & 0.0908 & 0.1288 & 0.1373 \\
\hline var & 0.0833 & 0.0106 & 0.0698 & 0.0439 & var & 0.0370 & 0.0083 & 0.0166 & 0.0077 \\
\hline
\end{tabular}

Tables 6.9-6.11 show that the average change difference in richness, evenness and diversity in the structure of the BE and GS of Auckland is bigger than the change difference exhibited by the richness, evenness and diversity in the structure of Nezu (Table 6-6). The structure of the BE of Auckland has changed more than the BE of Nezu (Table 6-7).

It is important to note that the analysis of variations and standard deviations together add information about the quality of change. Variations and standard deviations in the BE+GS (Table 6-6) and also in the BE of Auckland (Table 6-7) are higher than in the BE+GS (Table 6-6) and BE (Table 6-7) of Nezu. However variations and standard deviations of Nezu for GS are bigger than those of Auckland. This situation is linked with change in the richness of each structure. Less variation in the richness of a system can be linked with its more robust urban Panarchy that exhibits more resilience to change by maintaining its stability state. Variations are higher in Auckland when its structure is less heterogeneous than Nezu. BE and BE+GS of Auckland are less diverse than Nezu and perhaps for this reason they vary more than Nezu. Even though the quantity of change of GS in Auckland is bigger than in Nezu, it has changed less in 
richness, therefore it has varied less. This result could reinforce the hypothesis that more heterogeneous structures are more resilient and therefore their adaptation processes to change are softer than those found in less heterogeneous landscapes.

\subsubsection{Rate of change in the heterogeneity of Nezu and Auckland with and without Green Spaces}

Table 6-12. Rate of change in Nezu and Auckland BE and GS

\begin{tabular}{|c|c|c|c|c|c|c|c|c|c|}
\hline \multicolumn{5}{|c|}{ Nezu Rate of change BE+GS } & \multicolumn{5}{|c|}{ Auckland Rate of change BE+GS } \\
\hline & Richness & Evenness & Diversity & Mean & & Richness & Evenness & Diversity & Mean \\
\hline $\begin{array}{l}1956- \\
2003 \\
\end{array}$ & 0.0000 & 0.0020 & 0.0009 & 0.0010 & $\begin{array}{l}\text { 1966- } \\
2006\end{array}$ & 0.0063 & 0.0031 & 0.0022 & 0.0038 \\
\hline $\begin{array}{l}1932- \\
1956\end{array}$ & 0.0000 & 0.0117 & 0.0074 & 0.0064 & $\begin{array}{l}1931- \\
1966\end{array}$ & 0.0143 & 0.0001 & 0.0050 & 0.0065 \\
\hline $\begin{array}{l}1919- \\
1932\end{array}$ & 0.0769 & 0.0042 & 0.0156 & 0.0323 & $\begin{array}{l}1908- \\
1931\end{array}$ & 0.0238 & 0.0114 & 0.0121 & 0.0158 \\
\hline $\begin{array}{l}1888- \\
1919 \\
\end{array}$ & 0.0259 & 0.0010 & 0.0064 & 0.0111 & $\begin{array}{l}1882- \\
1908 \\
\end{array}$ & 0.0096 & 0.0024 & 0.0051 & 0.0057 \\
\hline Mean & 0.0257 & 0.0047 & 0.0076 & 0.0127 & Mean & 0.0135 & 0.0043 & 0.0061 & 0.0079 \\
\hline stdev & 0.0363 & 0.0049 & 0.0061 & 0.0137 & stdev & 0.0076 & 0.0049 & 0.0042 & 0.0056 \\
\hline var & 0.0013 & 0.0000 & 0.0000 & 0.0002 & var & 0.0001 & 0.0000 & 0.0000 & 0.0000 \\
\hline
\end{tabular}

Table 6-13. Rate of change in Nezu and Auckland BE

\begin{tabular}{|c|c|c|c|c|c|c|c|c|c|}
\hline \multicolumn{5}{|c|}{ Nezu Rate of change BE } & \multicolumn{5}{|c|}{ Auckland Rate of change BE } \\
\hline & Richness & Evenness & Diversity & mean & & Richness & Evenness & Diversity & Mean \\
\hline $\begin{array}{l}1956- \\
2003\end{array}$ & 0.0000 & 0.0026 & 0.0012 & 0.0013 & $\begin{array}{l}\text { 1966- } \\
2006\end{array}$ & 0.0000 & 0.0052 & 0.0008 & 0.0020 \\
\hline $\begin{array}{l}1932- \\
1956\end{array}$ & 0.0000 & 0.0138 & 0.0087 & 0.0075 & $\begin{array}{l}1931- \\
1966\end{array}$ & 0.0095 & 0.0005 & 0.0029 & 0.0043 \\
\hline $\begin{array}{l}1919- \\
1932\end{array}$ & 0.0513 & 0.0005 & 0.0120 & 0.0212 & $\begin{array}{l}1908- \\
1931\end{array}$ & 0.0317 & 0.0124 & 0.0141 & 0.0194 \\
\hline $\begin{array}{l}1888- \\
1919 \\
\end{array}$ & 0.0115 & 0.0031 & 0.0017 & 0.0054 & $\begin{array}{l}1882- \\
1908 \\
\end{array}$ & 0.0128 & 0.0005 & 0.0041 & 0.0058 \\
\hline Mean & 0.0157 & 0.0050 & 0.0059 & 0.0089 & Mean & 0.0135 & 0.0046 & 0.0055 & 0.0079 \\
\hline stdev & 0.0243 & 0.0060 & 0.0053 & 0.0087 & stdev & 0.0133 & 0.0056 & 0.0059 & 0.0083 \\
\hline var & 0.0006 & 0.0000 & 0.0000 & 0.0001 & var & 0.0002 & 0.0000 & 0.0000 & 0.0001 \\
\hline
\end{tabular}

Table 6-14. Rate of change in Nezu and Auckland GS

\begin{tabular}{|l|l|l|l|l|l|l|l|l|l|}
\hline \multicolumn{2}{|l|}{ Nezu Rate of change GS } & \multicolumn{4}{l|}{ Auckland Rate of change GS } \\
\hline & Richness & Evenness & Diversity & Mean & & Richness & Evenness & Diversity & Mean \\
\hline $\begin{array}{l}1956- \\
2003\end{array}$ & 0.0000 & 0.0001 & 0.0000 & 0.0000 & $\begin{array}{l}1966- \\
2006\end{array}$ & 0.0250 & 0.0034 & 0.0113 & 0.0132 \\
\hline $\begin{array}{l}1932- \\
1956\end{array}$ & 0.0000 & 0.0055 & 0.0038 & 0.0031 & $\begin{array}{l}1966 \\
195-\end{array}$ & 0.0286 & 0.0019 & 0.0115 & 0.0140 \\
\hline $1919-$ & & & & & $1908-$ & & & & \\
1932 & 0.1538 & 0.0154 & 0.0265 & 0.0653 & 1931 & 0.0000 & 0.0084 & 0.0059 & 0.0048 \\
\hline
\end{tabular}




\begin{tabular}{|l|l|l|l|l|l|l|l|l|l|}
$\begin{array}{l}1888- \\
1919\end{array}$ & 0.0690 & 0.0053 & 0.0204 & 0.0315 & $\begin{array}{l}1882- \\
1908\end{array}$ & 0.0000 & 0.0112 & 0.0078 & 0.0063 \\
\hline Mean & 0.0557 & 0.0066 & 0.0127 & 0.0250 & Mean & 0.0134 & 0.0063 & 0.0091 & 0.0096 \\
\hline stdev & 0.0731 & 0.0064 & 0.0128 & 0.0304 & stdev & 0.0155 & 0.0043 & 0.0027 & 0.0075 \\
\hline var & 0.0053 & 0.0000 & 0.0002 & 0.0009 & var & 0.0002 & 0.0000 & 0.0000 & 0.0000 \\
\hline
\end{tabular}

Tables 6-12, 6-13, and 6-14 show that the average rate of change in richness, evenness and diversity in the structure of the BE and GS of Nezu is bigger than the rate of change exhibited by the richness, evenness and diversity in the structure of Auckland. The structure of Nezu has changed faster than the structure of Auckland. Even though from 1956 to 2003 the urban landscape of Nezu has remained quite stable with a lower rate of change than Auckland, the rate of change of Nezu GS impacts on the whole system raising its rate of change.

Auckland GS and Nezu GS change faster than their respective BE. In the case of Nezu that has a lesser quantity of GS, changes in the GS are driven by changes in the BE. In the case of Auckland, a bigger rate of change in GS matches with a lower rate of change in BE and vice versa.

\subsubsection{Synthesis of the evolution of the heterogeneity of the urban landscapes of Nezu and Auckland}

Table 6-15. Comparison of heterogeneity, rate of change and change difference between Nezu and Auckland

\begin{tabular}{|l|l|l|l|l|l|l|l|l|l|}
\hline & \multicolumn{4}{|l|}{ Heterogeneity } & \multicolumn{3}{l|}{ Rate of change } & \multicolumn{3}{l|}{ Change Difference } \\
\hline & BE+GS & BE & GS & BE+GS & BE & GS & BE+GS & BE & GS \\
\hline Nezu & 1.3190 & 1.3430 & 1.2472 & 0.0127 & 0.0089 & 0.0250 & 0.1695 & 0.1612 & 0.2259 \\
\hline Auckland & 1.1403 & 1.1132 & 1.2218 & 0.0079 & 0.0079 & 0.0096 & 0.1757 & 0.1657 & 0.2277 \\
\hline
\end{tabular}

The structure of the built environment of the urban landscape of Nezu is more heterogeneous and has been changing less but faster than Auckland. The comparative analysis between Nezu and Auckland shows that more heterogeneous urban landscapes tend to experience less structural change in their richness and diversity than less heterogeneous urban landscapes. From an ecological resilience viewpoint, these results support the assumption that more diverse systems have a higher resilience capacity than less diverse systems and 
for this reason they will tend to buffer change more effectively, within and across scales, experiencing less change in their structure and heterogeneity.

The heterogeneity of Nezu is based in the quality of its BE, particularly in the diversity and richness of its ST system, while the heterogeneity of Auckland relies on the diversity and richness of its GS that is bigger than the average heterogeneity of its BE. GS in Auckland and Nezu have changed more in quantity and faster than their respective BEs, and for this reason the heterogeneity of the urban landscape of Auckland has been more affected in terms of change difference. The relationship between the rate of change and the change difference in Nezu exposes its tendency to change quickly without causing major modifications in its own structure due to the complexity and heterogeneity of its $\mathrm{BE}$ that can be more resilient to changes in GS.

\subsection{Results}

Results sustain the hypothesis that more heterogeneous landscapes are more diverse and their structures should tend to change less. These facts prove that assumptions stated in the TDH can be considered to apply when analyzing the resilience of urban landscapes.

The analysis of Nezu and Auckland using a resilient assemblage approach proves that the use of the methodology and method proposed generate quantitative and qualitative information for understanding the resilience of the built environment of urban landscapes in relationship with their complexity and adaptability.

The quantification of discontinuities in the structure of an urban landscape permits an insight into the resilience of an urban landscape. The observation of change in the diversity and richness of two systems is an important step towards the measurement of general and specific resilience in urban landscapes.

A cluster analysis permits the bounding of information in large data bases, a factor that make data management a lot easier and that also allows a different view of a system based on a few key variables. Statistical variables like rate of change, growth, change difference, standard deviation and variance produce key information in the measurement of morphological change. At the 
same time calculations of ecological indices like diversity, evenness, and richness contribute to the quantitative assessment of urban landscapes and to the challenge of measuring the heterogeneity of a landscape in time and space. The method applied in this research is highly focused on creating a parameter to analyse the rhythm of change of evolutionary forces in urban landscapes, their self-organization processes, and interrelationships between elements that structure the urban landscape.

By using the proposed methodology it is possible to realize which group of sizes and specifically what ranges are more persistent and which ones tend to change more frequently. This type of information gives important clues about the morphology of key elements that structure the identity of the built environment and characterize the stability state of the urban landscape. The awareness of what PL, BL, ST sizes are more resistant and resilient to change is useful data that could be incorporated in the design process of new developments and projects.

Green spaces contribute to the complexity and diversity of the urban landscape. The results show that in the case of both Auckland and Tokyo GS behave in an independent way from the levels of the built environment. Namely, changes in diversity of GS do not necessarily follow the same developments in the built environment. The heterogeneity of Auckland GS and Nezu GS changed faster and more in quantity than their respective BE. It can be inferred that they represent another level in the urban landscape. Quantities of GS were shrinking through time but the diversity exhibited by GS was changing in an alternative manner. The contribution of green spaces to overall diversity of the assemblage was linked with the richness of their structures and not necessarily with their quantities. In this manner they contribute to the complexity of the system and to its diversity by adding aggregation and discontinuities to the whole structure.

The richness and diversity of domestic GS is restrained by the heterogeneity of the BE. The diversity produced by domestic GS, at small scales, depends on the diversity of building footprints, plots, and block sizes at the same scale. Parks and other bigger green areas contribute to the total amount of GS but not necessarily to its diversity. Auckland contains more and bigger green public and private spaces than Nezu, but the BE of Auckland is less rich and diverse 
than Nezu, therefore its GS are also less heterogeneous. The insertion of green public spaces in BEs that are more diverse, like Nezu, would be a more effective way to enhance heterogeneity. This result should be a consideration for designers and planners that are interested in the resilience of urban landscapes. Policies and designs that acknowledge the relationship between building footprints, plots size and green spaces is necessary in order to enhance the heterogeneity of GS at small scales.

The heterogeneity of the BE can be enhanced or lessened by the heterogeneity of GS. Auckland GS are more heterogeneous than its BE, therefore GS helps to enhance its richness and diversity. Nezu GS are more heterogeneous than Auckland GS but less rich and diverse than its own BE, and for this reason they lessen the heterogeneity of the entire system. According to the performance of the urban landscapes of Nezu and Auckland, when the GS is less rich and diverse than its correspondent $\mathrm{BE}$, changes in the heterogeneity of the BE should impact on its GS; however, changes in a BE that is less rich than its own GS, will not necessarily impact on the structure of GS. Urban landscapes with important heterogeneity in BE and GS would hold rich and diverse structures that are fairly stable in the face of unpredictable disturbances.

GS contributes to the resilience capacity of the urban landscape for buffering change in its built environment. The analysis of the evolution of Nezu and Auckland shows that alterations in the composition of their GS affected the structure, composition and stability of both built environments. The observations sustain the hypothesis that green spaces have the role of buffering spaces that tend to stabilize the non-linear dynamics of transformations happening in the built environment of urban landscapes. Based on results obtained, it is fair to consider GS as a resilience capital of the built environment. If GS and other un-built (in-between) spaces are part of a resilience capital they are also linked with the production of opportunities for the urban landscape. In this case opportunities mean the necessary space to make the BE and GS more or less heterogeneous and consequently to increase or decrease its resilience capacity. In any case, the structure of the urban landscape, the quantity and quality of its elements and its evolutionary dynamics of change, are all associated with the production of opportunities and novelties. 
The assessment of the evolution of the structure of green spaces can provide important information about the stability of an urban landscape and allow some insight into the resilience behaviour of a built environment. The potential of these findings are linked with the possibility of quantifying the resilience capacity of a socio-ecological landscape by analyzing changes in its built environment and also of using a resilience approach to designing architectural and urban interventions in urban landscapes.

\subsection{Conclusion}

This analysis only shows one aspect of the stability state of the urban landscapes of Auckland and Nezu related with the evolution of their respective town plans, particularly with the dynamics of change of the built environment and its resilience to internal and external disturbances, which it is hoped contribute to assessing the resilience capacity of socio-ecological landscapes. For these reasons the target of the research was not focused on the discovery of morphological patterns or types. However, if this method were to be related with timelines and the identification of events and processes that have an impact on the urban landscape a typo-morphological research could be established.

The analysis of data using more developed statistical and mathematical tools along with a deeper understanding of ecological concepts could also bring a new insight into quantitative and qualitative urban analysis related with resilience.

Resilience thinking becomes useful for dealing with the unpredictable nature of socio-ecological change, through identification of spaces for resilience within urban areas. The understanding of space as a capital asset within cities is a key contribution of this research to the future analysis of the resilience of urban landscapes. Particularly significant is the fact that opportunities in urban landscapes are not only created by new architectural and urban interventions but are also contained in the structure of resilient spaces and in the future evolution produced by the interaction of elements of the same urban landscape. These systems of spaces in a process of becoming are the arena of opportunities and risks, and for this reason they are spaces for resilience. Spaces for resilience are natural resources that can be understood as a socio-ecological service 
produced and consumed by socio-ecological landscapes. The results of this research confirm the idea that the built environment is not just a consumer of socio-ecological services but is also a generator of spaces for resilience. For these reason the built environment becomes an essential element in analysing the resilience of cities (Garcia \& Vale, 2012).

The production of spaces for resilience is linked with the way in which the discrete evolution of a particular built environment occurs. Resilience is an attribute that allows complex systems to be changed in order to be adaptive. For this reason the transformations of the built environment of a socio-ecological landscape are susceptible to being analyzed through the theory of ecological resilience. The increased urbanization of the global population, resource shortages and the idea of one planet living suggest a need to understand how cities truly behave and evolve. The ability of a complex system to adapt to change that comes from internal and external pressures is the essence of creating a system than can sustain itself in the long term.

Discovering different levels of heterogeneity can be useful for defining how much diversity and richness is needed in order to consider an urban landscape heterogeneous enough. At the same time the development of other comparative analysis between different urban landscapes could produce more information about the resilience dynamics of change. 


\section{Conclusions}

\subsection{Introduction}

This chapter highlights contributions made by the thesis research to the analysis of urban landscapes. The first group of results is related to the theoretical evidence achieved by creating a philosophical approach to translating topics from ecology to the analysis of urban landscapes. The criteria for assessment developed from the interdisciplinary set of concepts assembled in the first three chapters showed evidence that the ecological resilience theoretical background could be translated and applied in urban studies. The second group of results is related with the possibilities of applying the ecological resilience methodology to the measurement of change in the built environment of urban landscapes. Due to the complexity of and information needed for the calculations, the methodology was tested in different steps. The possibilities exploited in this research are not the only way of assessing resilience but they represent a set of criteria that prove that is possible to measure resilience in urban landscapes. Each step emphasizes and evaluates different aspects of the methodology in order to analyse the potential and implications of the instrumental theory when it is applied to concrete case studies.

\subsection{Answering the research question}

The main research question was: how is it possible to apply the ecological resilience theoretical framework to the analysis of urban landscapes?

The hypothesis was based on the assumption that the application of ecological resilience to the analysis of urban landscapes could be approached through assessing the dynamics of change in the morphology of built environments.

Through the assessment of the dynamics of change in the morphology of the built environment performed in chapters 4, 5 and 6, in accordance with the theoretical background developed in chapter 2 and 3, theoretical and practical evidence of how to apply the ecological resilience theoretical framework to the analysis of urban landscapes was found. The sets of results, grouped as 
theoretical and practical evidence, contribute to answering the research question and to validating the hypothesis as stated.

The theoretical evidence can be grouped around the idea that the application of ecological resilience to the analysis of urban landscapes is possible by implementing a complex methodological approach that merges ecology, morphogenetics and assemblage theory.

The practical evidence found can be grouped around the idea that the measurement of certain dynamics of change (in the evolution of urban landscapes, in the identity of place, and in the heterogeneity of the built environment) gives information about possible ways of applying the ecological resilience theoretical framework to the assessment of the resilience capacity of urban landscapes. In this way, theoretical and practical conclusions give evidence of how to apply the resilience theoretical framework to urban landscapes.

\subsection{Theoretical evidence: thinking about how to apply ecological resilience to the analysis of urban landscapes}

The development of the ontology and philosophical approach, carried out in the first three chapters, sustains the idea that the translation of key concepts from ecology to urbanism is possible and that this is supported in literature and research done in alternative fields of study. Therefore the criterion for assessment derived from the complex methodological approach of the thesis is a theoretical construct that establishes the starting point for thinking about how to apply ecological resilience to urban landscapes.

A morphogentic understanding clarifies the elements of the built environment that have to be analysed in order to understand the structure and evolution of urban landscapes. The theoretical background of morphogenetics (Whitehand, 1981) and the theoretical framework of ecological resilience and its methodology (Resilience Alliance, 2007a) when merged and implemented together become useful tools with which to analyse the dynamics of change in the built environment. These findings demonstrate that the complex 
methodological approach creates a territory of intersection between morphogenetics and ecological resilience that can increase the understanding of the dynamics of change in urban landscapes.

The implementation of the complex methodological approach to the assessment of change in two built environments using original methods can be used to give evidence of how to apply ecological resilience to the analysis of urban landscapes. Therefore the methods are also part of the results of this thesis.

The application of ecological resilience to urban landscapes does not demonstrate that ecosystems are equal to urban landscapes but only that the complexity of ecosystems and their dynamics of change related to adaptation and resilience can be compared with the complexity and dynamics of change observed in urban landscapes. From this systemic viewpoint, the comparison between the dynamics of change of ecosystems and urban landscapes is founded in the fact that both systems can be analysed as complex adaptive systems. The analysis in chapters 4, 5 and 6 provide evidence that the dynamics of change of urban landscapes show properties like non-linearity, systemic interactions, historical sequence and spatial relationships (Holling \& Goldberg, 1971, p. 226) that are characteristics of complex adaptive systems. Therefore, methodologies created to analyse the resilience dynamics of change in ecosystems should be valid to be applied to the study of urban landscapes.

The groups of concepts used in the construction of the theoretical background of the thesis and the methods proposed could be used as a foundation for the development of an instrumental theory to explain and assess change in the built environment of urban landscapes. Moreover the way of thinking about the resilience of urban landscapes proposed in this thesis can be used, through the implementation of the methodology and methods proposed, to conduct comparative analysis in urban morphology and ecology. 
7.4 Practical evidence: ways of applying ecological resilience to the analysis of urban landscapes

\subsubsection{Ecological resilience can be applied to the analysis of urban landscapes by assessing the dynamics of change in key elements of the built environment}

The analysis of the case study of the east side of Auckland CBD in chapter 4 supports the assumption that adaptive dynamics of change in urban landscapes generate a dynamic hierarchy (Panarchy). Through the quantitative assessment of timelines, a Panarchy can be observed in the way that key elements of the built environment of urban landscapes (blocks, plots, streets and building footprints) are structured, interacts and change. The analysis of the dynamics of change in the morphology of the built environment of Auckland shows that the structure of its urban landscape can be understood and related to spatio-temporal processes of change that are also found in Panarchy. Therefore the study supports the hypothesis that the resilience capacity of urban landscapes can be assessed by analysing changes in the morphology of the built environment. By testing the hypothesis, results exhibit evidence of one possible way of applying the ecological resilience theoretical framework to the analysis of urban landscapes.

The case study in chapter 4 shows that elements of the urban landscape change at different rates and occupy different levels in the hierarchy of which they are a part. The urban system holds discrete scales each one having its own equilibrium point that may differ from other scales. In this way the urban system is shown to have multiple equilibrium points in its structure across scales. At the same time each element of the built environment is changing and altering at its own dynamic of change. This fact demonstrates that within scales, every level has its own equilibrium point that is not fixed but moving in time, pushing each element to work in more than one stability state. The complex structure of the built environment, exposed in the hierarchy of multiple scales and levels that it contains, along with its multiple equilibrium points, manifests the possibility that changes at one scale may or may not affect the stability at larger scales. This fact 
can be used to support assumptions about how adaptability occurs in resilience dynamics. Particularly the point of the hypothesis in which it is stated that transformations in the morphology of the urban landscape at one scale might or might not produce structural changes at larger scales.

Results of the timeline analysis in Auckland also reveal that the built environment of urban landscapes is complex and adaptive. Results also show that the assemblage of methods from ecological resilience and urban morphology can produce important information about the dynamics of change of the urban landscape.

The different rates of change of the complexes of elements analysed in the evolution of the urban landscape of Auckland show no evidence of responding to a single controlling event that affects all scales and processes at the same time and scale. Building footprints, plots, blocks, streets show different responses and are affected, as explained in the previous paragraph, by different contextual events and processes. The stability of a built environment is therefore the product of non-linear responses and the interplay between different processes of change that produce responses that are adaptations at different rates, through multiple scales. Consequently, it is possible to state that the capacity of the built environment for being adaptive to change at broader scales depends on its capacity to buffer change within and across smaller scales. The case study analysed demonstrates that the heterogeneity of the structure of an urban landscape is an essential attribute in assessing its resilience capacity. Considering all these results, it is possible to affirm that the implementation of resilience thinking into the analysis of urban landscapes is possible and can be done by analysing the morphology of the built environment.

The proposal that an urban landscape, and particularly its built environment, is structured like an urban Panarchy implies that its management and interventions have to contemplate the understanding, analysis and evaluation of more than one scale, element and process of the system at the same time, and as part of the same phenomenon, even if the processes studied are contradictory. At the same time, this situation presents a challenge to its government because the urban Panarchy structure shows that the built environment demands a complex understanding of the history and evolution of each system analysed. But most 
importantly, the complexity and self-organizing capacity of the system demonstrates that policies, laws, urban designs, and other formal institutional responses to governing and to controlling the shape of the built environment will always have to deal with a margin of unpredictability, anarchy, and chaos that together and separately play an important role in the resilience and evolution of the urban form of cities.

\subsubsection{Ecological resilience can be applied to the analysis of urban landscapes by comparing changes in key elements of the built environment against persistence or shifts in the identity of place.}

The analysis of Nezu shows that the urban landscape produces patterns of aggregations and discontinuities that can be observed as clusters of Blocks, Plots, and Building Footprint size classes. These findings consolidate the proposal that urban landscapes are complex adaptive systems organized in an urban Panarchy, which is responsible for the production of a heterogeneous landscape.

The presence and emergence of clusters of size classes in the built environment permits abstraction of the urban landscape in order to understand its structure. Observations about changes in that structure give information about the general performance of the system, which allows structural behaviours to be compared against particular processes occurring at focal scales. These comparisons are significant for understanding the complexity of complex adaptive systems like the built environment of Nezu. Moreover it gives the possibility of quantifying change at different scales, a basic necessity for assessing the resilience dynamics of the urban landscape.

The results explicitly show that in order to analyse the complexity of urban landscapes, the dynamics of change in elements of a built environment should be attended to in parallel with the dynamics of change of the structure that contains these elements. The demonstration of this statement can be found in the analysis of Nezu where the rate of change in elements at different scales differs from change in the structure and responses in the urban landscape. These cross scale dynamics can be observed in the urban landscape of Nezu. For example, change in the built environment of Nezu caused by fires or other socio- 
ecological threats did not produce critical change in the identity of the place. Different scales in the urban Panarchy of Nezu buffer change that could happen at a particular scale, for example, at the scale of plots where the loss of building footprints might be important.

The theoretical framework of ecological resilience can be applied to an analysis of the evolution of the built environment, in this case through the analysis of the stability state of the identity of a place. This way of thinking avoids traditionalist approaches to the built environment but concentrates on its structure and how the physical environment of a society, through interrelationships within and across scales, contributes to its identity. From a resilient assemblage approach, the identity of the physical structure of an urban landscape, like the built environment, is a property that results from the complexity and adaptability of urban landscapes. This identity becomes not only the zone of discussion about the development of the character of a system, and opportunities to maintain or to change that character, but also the parameter with which to analyse the way in which a society occupies, uses and exploits a territory.

Investigations about identity of a place can produce information about the state of the socio-ecological landscape that contains it. The assessment of the built environment of an urban landscape also provides information about its non-built environment. However, although this was not formally analysed in this research, it can be theoretically inferred, particularly in the analysis of green spaces in Nezu and Auckland as described in chapter 6. The persistence of the identity of a place demonstrates that certain dynamics of change taking place at different scales in the built environment can contribute to sustaining the stability of the entire system instead of making it collapse. The persistence of the identity of a characteristic urban landscape, like Nezu, proves that the resilience capacity of the structure of a built environment to absorbing change at smaller scales while keeping its bigger scales stable is linked with the persistence of the identity of that urban landscape.

The unity found in the identity of an urban landscape is not related with a uniformity or homogeneity of elements. Contrary to this idea, the persistence of an identity is connected with change, heterogeneity and diversity of elements 
that enhance resilience and give robustness to the structure of the urban landscape. Identity not only depends on the characteristics of the built environment of the place itself, but at a different scale will depend upon the characteristics and interrelationships with the built environment of other places. Identity of the place has its own complexity that is based on relationships between a society, land and its built environment. That is why it is a suitable parameter for expressing the quality of the place as a whole and can be applied to produce a panorama of the resilience capacity of a built environment. Identity of the place also provides information about the socio-ecological system, which is culturally specific. Since identity is traditionally indigenous, it also allows the method to be applied to different places.

The idea of linking the analysis of the identity of place with the stability state of the built environment of urban landscapes in order to assess the resilience of a socio-ecological landscape can form a concrete way of relating the dynamics of a system with a quality of the built environment. The importance of this interdisciplinary collaboration is that the analysis of identity is the way to define a place as possessing different levels of resilience capacity. Since urban and ecological systems have a difference based upon the importance of their elements, identity proves to be a main parameter in its resilience capacity. Within this viewpoint, quantitative measurements are related to the characteristics of a built environment and qualitative attributes are introduced through the study of identity.

\subsubsection{Ecological resilience can be applied to the analysis of urban landscapes by measuring changes in the heterogeneity of the built environment of urban landscapes}

The assessment of relative resilience in urban landscapes by quantifying the heterogeneity, diversity and evenness of the structure of the built and nonbuilt environment, represented as an assemblage of aggregations and discontinuities, produces concrete results and information about the resilience of the socio-ecological system. These results permit assessment of the adaptive 
capacity of an urban landscape by establishing a comparison of the structure of an urban landscape before and after critical events.

The method facilitates the evaluation of different evolutionary processes in discrete urban landscapes, a fact that can benefit the development of comparative studies and form an advance in resilience knowledge in urban landscapes. Finally the methodology shows that the measurement of resilience is not an obscure science, moreover it is possible to obtain precise information.

Results from the comparison between Nezu and Auckland show quantities of elements in a built environment are important, but quality, diversity and organization of these elements are also essential for the resilience capacity of the structure of an urban landscape.

The perception of an urban landscape, which is the base for the creation of various metaphors, analogies and urban discourses, is different from its structure; therefore this perception gives partial and sometimes superficial information about the state of an urban landscape. The fact that in one place it is possible to perceive many green spaces does not imply that the structure of these green spaces can provide diversity while enhancing the heterogeneity and resilience of the system.

These quantitative facts are linked with the identity of a built environment and even more with the perception of heterogeneity in an urban landscape. A negatively perceived chaotic, dispersed and extremely diverse built environment, like a gas station, a hut, and a high-rise building next to a highway, perhaps has a rich and diverse structure that can hold an important resilience capacity to absorb change. From a resilient assemblage approach, this landscape has an identity, a stability state and a resilience capacity that can be enhanced or decreased in relationship with specific or general goals in a development. From a traditional urban design viewpoint it is a mess.

The interpretation of quantitative results about the resilience capacity of a system has to be put in context in order to mean something, otherwise its implications for designers and managers will be minimal. Resilience is an attribute that has to be used and aligned to a broader picture. The theoretical background and results obtained question the possibility of achieving resilient cities as a whole and final state of a system because the resilience of something 
will always be linked with something else that cannot include the totality of the system. Another factor that sustains the impossibility of making a whole city resilient to everything is the fact that necessities change in time and space. An evolutionary approach to resilience is perhaps the most difficult task in understanding the importance of resilience.

The aim of this thesis was not to exploit design possibilities or to suggest policies, mainly because these will depend on emergent necessities and people creating complex questions that deserve this kind of complex analysis. In a metaphorical way it is reasonable to say that the object of this thesis was to show how to build a hammer and the fact that such a hammer is worth something. Users coming after will improve its design and discover new applications for it, accepting that the same hammer can be used to build beautiful houses and to kill people. Such further uses are beyond the scope of this thesis.

\subsection{Weaknesses}

The challenge in the application of ecological resilience to urban landscapes is that the theoretical framework is not a theory, while its methodology is neither too scientific nor too heuristic. Due to these weaknesses the application of ecological resilience in fields other than ecosystems becomes complicated because the lack of a theory makes the implementation of its methodology not rigorous enough for scientists and very complex for general designers and practitioners. These gaps were tackled in this research by developing an epistemological approach where the diversity of consolidated knowledge from different fields can give a support to each hypothesis and at the same time provide clues to and justification for its application to the analysis of urban landscapes.

Even though vulnerabilities in the application of the methodology and in the method used to make measurements were detailed in chapters 5 and 6 , they do not relate to the overview of the problematic but rather to specific issues. Because the practice of the methodology proposed a challenge to the linear way of making observations, there is a problem in analysing parallel and, sometimes, contradictory results as a part of the same research. At the same time the quantity of data is large and calculations have more than one step, increasing the 
risk of making mistakes. Results need to be assessed by using different criteria coming from discrete fields, hence increasing the possibility for misunderstandings. Therefore, results are difficult to read objectively. The design of processes or a protocol to make research in this field will decrease the margin of error and also contribute to identifying where the major problems of the methodology and method are to be found.

Perhaps the biggest weakness and major task for future research in this field is to convince managers, designers, and institutions that it is important to understand how a system works before trying to intervene in it or to predict specific outputs. Therefore one challenge is to link studies in the resilience of urban landscapes with the cost of not being adaptable while keeping away from engineering approaches that try to find silver bullets to very specific issues, such as solving the problems of a whole city. Moreover, it might be interesting to use resilience approaches to increase curiosity about the necessity of knowing more about the way in which urban landscapes and systems work, instead of assuming that they just change.

\subsection{Discussion and opportunities for further research}

Resilience thinking becomes useful for dealing with the unpredictable nature of socio-ecological change, through identification of spaces for resilience within urban areas. The understanding of space as a capital asset within cities is a key contribution of this research to the future analysis of the resilience of urban landscapes. Particularly significant is the fact that opportunities in urban landscapes are not only created by new architectural and urban interventions but are also contained in the structure of resilient spaces and in the future evolution produced by the interaction of elements of the same urban landscape. These systems of spaces are the arena of opportunities and risks, and for this reason they are spaces for resilience. Spaces for resilience are natural resources that can be understood as a socio-ecological service produced and consumed by socio-ecological landscapes. The results of this research confirm the idea that the built environment is not just a consumer of socio-ecological services but is also a generator of spaces for resilience. For these reason the built environment 
becomes an essential element in analysing the resilience of cities (Garcia \& Vale, 2012).

The production of spaces for resilience is linked with the way in which the discrete evolution of a particular built environment occurs. Resilience is an attribute that allows complex systems to be changed in order to be adaptive. For this reason the transformations of the built environment of a socio-ecological landscape are susceptible to being analysed through the theory of ecological resilience. The increased urbanization of the global population and resource shortages, and the idea of one planet living suggest a need to understand how cities really behave and evolve. The ability of a complex system to adapt to change that comes from internal and external pressures is the essence of creating a system than can sustain itself in the long term (B. Walker \& Salt, 2006).

A first suggested research project using the idea of spaces for resilience could be oriented to producing a model that allows the understanding, definition and implications of the resilience of urban landscapes. Therefore the aim of this kind of research would be the production of knowledge about the emergence of alternative systems of spaces which result from change but which give an opportunity for the system to reorganise and to self-organize. The proposition is that the presence in a city of spaces for resilience will enhance the resilience capacity of the whole system. This research could discover what these spaces are and the potential for application of the approach to all urban areas.

The hypothesis is that there is a relationship between the patterns and diversity in the way buildings occupy the urban landscape and the adaptive capacity of the latter to face natural and cultural hazards. Moreover, quantification of the resilience capacity of cities can be approached by applying a resilient assemblage approach to the analysis of urban landscapes, and also by measuring with mathematical models, morphological and functional transformations in the built environment. Only by using this as a base can a predictive model of how an urban landscape might change when affected by a natural or cultural hazard then be constructed. This predictive model could the final goal of this further research. 
Such a model is about strengthening the urban landscape as a whole and its ability to withstand and respond positively to traumatic events, such as earthquakes. What the model could do is to show how likely a city or town could function normally following a hazardous event. A quicker recovery is likely to be a lower cost recovery, both in monetary terms as the local economy can get back to normal quickly, but also in personal, social and health sector terms by reducing the stresses that long term uncertainty about when things will get back to normal impose on the people involved in a hazard event.

A second group of potential investigations comes from merging the resilient assemblage approach and ecological footprint analysis. The subject would be the role that urban redevelopment plays in the resilience of cities. This is because redevelopments can be turning points in urban evolution, either exploiting opportunities that have emerged in the evolution of a city or creating scenarios with lowered resilience. This proposal would look at a critical point in the evolution of an urban landscape, which is its recovery after a collapse. The aim is to see whether urban landscapes that are rebuilt as close to the original as possible, are equally, more or less stable than cities where underlying patterns of elements of the urban landscape have been fundamentally changed in the rebuilding.

The morphogenetic technique could be used to look at socio-cultural impact on the urban fabric through measurement of identity. Material and energy flows can be measured using an ecological footprint approach. The research would first need to establish a pre-event pattern of evolution and then go on to examine the evolution post re-building to look for changes in the urban morphology. The aim would be to compare the responses of different urban configurations to specific crises and to analyse the performances and evolution of these transformed urban landscapes. This research would again facilitate the evaluation of the resilience capacity of built environments to cope with unpredictable change and contribute to the prediction of which kind of urban structures, and physical interventions in these structures, would be more successful in adapting to future unpredictable changes. Building on findings done in this thesis along with research done in ecological footprints (R. Vale \& Vale, 2009), such a project would for the first time simultaneously track the impact on 
natural systems through measurement of the land and material resources that have gone into the rebuilding and subsequent evolution of the case study area. The aim would be to understand more about the resilience capacity of an urban system and what happens when human 'development' interacts with the process of urban evolution. This merging of the disciplines could lead to a greater understanding of how urban redevelopment affects the resilience of the urban environment. 


\section{Bibliography}

Adger, W. N., Hughes, T. P., Folke, C., Carpenter, S. R., \& Rockstrom, J. (2005). SocialEcological Resilience to Coastal Disasters. Science, 309(5737), 1036-1039.

Alberti, M. (2009). Advances in urban ecology: integrating humans and ecological processes in urban ecosystems (1st pbk. ed.). New York: Springer.

Alberti, M., \& Marzluff, J. M. (2004). Ecological resilience in urban ecosystems: Linking urban patterns to human and ecological functions. Urban Ecosystems, 7(3), 241265.

Alberti, M., Marzluff, J. M., Shulenberger, E., Bradley, G., Ryan, C., \& Zumbrunnen, C. (2003). Integrating humans into ecology: opportunities and challenges for studying urban ecosystems. BioScience, 53(12), 1169-1179.

Allan, P., \& Bryant, M. (2011). Resilience as a framework for urbanism and recovery. Journal of Landscape Architecture, 6(2), 34-45.

Allen, C. R., Gunderson, L., \& Johnson, A. (2005). The Use of Discontinuities and Functional Groups to Assess Relative Resilience in Complex Systems. Ecosystems, 8(8), 958-966.

Allen, C. R., Forys, E. A., \& Holling, C. S. (1999). Body Mass Patterns Predict Invasions and Extinctions in Transforming Landscapes. Ecosystems, 2(2), 114-121.

Allen, C. R., \& Holling, C. S. (2010). Novelty, adaptive capacity, and resilience. Ecology and Society 15(3), 15. Retrieved from http://www.ecologyandsociety.org/vol15/iss3/art24/

Allen, C. R., \& Holling, C. S. (2008). Discontinuities in ecosystems and other complex systems. New York: Columbia University Press.

Allen, P. (1997). Cities and regions as self-organizing systems: models of complexity. Australia: Gordon and Breach Science Publishers.

Anonymous (Cartographer). (1988). Kantōdaishinsai-shi: Shashin to chizu to kiroku de miru, 1987 - 1988-nen [Great Kanto Earthquake: View of photos and maps and records,1987 -1988].

ARUP (2012). Visions of a resilient city. Retrieved from http://www.arup.com/Home/Publications/Visions_of_a_Resilient_City.aspx.

Batty, M. (2010). Towards a new science of cities. Building Research \& Information, $38(1), 12$

Bessey, K. M. (2002). Structure and dynamics in an urban landscape: toward a multiscale View. Ecosystems, 5(4), 360-375.

Birch, E., \& Wachter, S. (2006). Rebuilding urban places after disaster: lessons from Hurricane Katrina The city in the twenty-first century (pp. xii, 375). Philadelphia: University of Pennsylvania Press. 
Braudel, F. (1969). Écrits sur l'histoire. Paris: Flammarion.

Braudel, F. (1977). La Méditerranée : l'espace et l'histoire. Paris: Arts et métiers graphiques.

Braudel, F. (1980). On history Chicago: University of Chicago Press.

Bush, G., \& Council., A. C. (1971). Decently and in order; the government of the City of Auckland 1840-1971: the centennial history of the Auckland City Council. Auckland: Collins for the Auckland City Council.

Caniggia, G. (2001). Architectural composition and building typology: interpreting basic building. Firenze: Alinea.

Carpenter, S., Walker, B., Anderies, J. M., \& Abel, N. (2001). From Metaphor to Measurement: Resilience of What to What? Ecosystems, 4(8), 765-781.

Cash, D. W., Adger, W., Berkes, F., Garden, P., Lebel, L., Olsson, P., . . Young, O. (2006). Scale and cross-scale dynamics: governance and information in a multilevel world. Ecology and Society, 11(2), 12. Retrieved from http://www.ecologyandsociety.org/vol11/iss2/art8/

Catedra Manteola, S., Turrillo (Ed.). (2006). Contexto. Buenos Aires: nobuko.

Certeau, M. d. (1984). The practice of everyday life. Berkeley: University of California Press.

Chang, Y., Wilkinson, S., Potangaroa, R., \& Seville, E. (2010). Resourcing challenges for post-disaster housing reconstruction: a comparative analysis. Building Research \& Information, 38(3), 247-264.

Chang, Y., Wilkinson, S., Potangaroa, R., \& Seville, E. (2011). Donor-driven resource procurement for post-disaster reconstruction: Constraints and actions. Habitat International, 35(2), 199-205.

Chen, Y.-Z. (2004). Edgar Morin's paradigm of complexity and Godel's incompleteness theorem. World Futures: Journal of General Evolution, 60(5), 421 - 431.

Clement, G. (2007). Manifiesto del tercer paisaje (M. Pla, Trans. gustavo Gilli ed.). Barcelona: Editorial Gustavo Gilli.

Colding, J. (2007). 'Ecological land-use complementation' for building resilience in urban ecosystems. Landscape and Urban Planning, 81(1-2), 46-55.

Conzen, M. R. G. (1960). Alnwick, Northumberland: A Study in Town-Plan Analysis. Transactions and Papers (Institute of British Geographers)(27), iii-122.

Conzen, M. R. G. (1981). The morphology of towns in Britain during the industrial era (N. K. Conzen \& M. P. Conzen, Trans.). In W. J. W. R. (Ed.), The Urban landscape: historical development and management. Papers by M.R.G. Conzen (pp. vii, 166). London: New York: Academic Press.

Council, A. R. C., \& team, S. a. E. R. a. M. (2010). A brief history of Acukland's urban form (Vol. 1, pp. 27). Auckland: Auckland Regional City Council. 
Curk, T., Demšar, T., Xu, Q., Leban, G., Petrovič, U., Bratko, I., . . Zupan, B. (2005). Microarray data mining with visual programming. Bioinformatics, 21(3), 396398.

Daniels, I. (2010). The Japanese house : material culture in the modern home. Oxford: Berg.

De Landa, M. (2006). A new philosophy of society : assemblage theory and social complexity. London; New York: Continuum.

Deleuze, G., \& Guattari, F. (1987). A thousand plateaus: capitalism and schizophrenia. Minneapolis: University of Minnesota Press.

Deleuze, G. (1994). Difference and repetition. New York: Columbia University Press.

Dovey, K. (2010). Becoming places: urbanism/architecture/identity/power. London; New York: Routledge.

Elmqvist, T., Colding, J., Barthel, S., BorgstrÖM, S., Duit, A., Lundberg, J., . . B Bengtsson, J. (2004). The Dynamics of Social-Ecological Systems in Urban Landscapes: Stockholm and the National Urban Park, Sweden. Annals of the New York Academy of Sciences, 1023(1), 308-322.

Evan, D. G. F., \& Warren, M. (2004). Identifying the secure city: research to establish a preliminary framework Canadian Journal of Urban Research, 13(1), 89-99.

Fleischhauer, M. (2008). The Role of Spatial Planning in Strengthening Urban Resilience. In H. Passman \& I. Kirillov (Eds.), Resilience of Cities to Terrorist and other Threats: learning from 9/11 and further research issues (pp. 273-298): Springler.

Folke, C., Carpenter, S., Walker, B., Scheffer, M., Elmqvist, T., Gunderson, L., \& Holling, C. S. (2004). Regime shifts, resilience, and biodiversity in ecosystem management. [Review]. Annual Review of Ecology Evolution and Systematics, 35, 557-581.

Frazer, J. (1995). An evolutionary architecture. London: Architectural Association.

Gallopín, G. C. (2006). Linkages between vulnerability, resilience, and adaptive capacity. Global Environmental Change, 16(3), 293-303.

Garcia, E., Muminovic, M., Vale, B., \& Radovic, D. (2012). The resilience of an assembled identity. Paper presented at the Place making and identity: rethinking urban approaches to built environement, Jakaarta.

Garcia, E., \& Vale, B. (2012). Spaces for urban resilience. Paper presented at the 1st International Conference on Urban Sustainability and Resilience, London.

Garcia, E., Vale, B., \& Allan, P. (2011). Before multi-hazards: a methodology to analyze the resilience of urban landscapes. Paper presented at the 7 th APRU Research Symposium on Multi-hazards around the Pacific Rim. Physical and human dimensions of natural hazards: from research to practice, Auckland.

Garmestani, A. S., Allen, C. R., Mittelstaedt, J., Stow, C., \& Ward, W. (2006). Firm size diversity, functional richness, and resilience. Environment and Development Economics, 11(04), 533-551. 
Garmestani, A. S., Allen, C. R., \& Bessey, K. M. (2005). Time-series analysis of clusters in city size distributions. Urban Studies, 42(9), 1507-1515.

Garmestani, A. S., Allen, C. R., \& Gallagher, C. M. (2008). Power laws, discontinuities and regional city size distributions. Journal of Economic Behavior \& Organization, 68(1), 209-216.

Gaston, K., Warren, P., Thompson, K., \& Smith, R. (2005). Urban Domestic Gardens (IV): The Extent of the Resource and its Associated Features. Biodiversity \& Conservation, 14(14), 3327-3349.

Geddes, P. (1915). Cities in evolution; an introduction to the town planning movement and to the study of civic. London: Williams \& Norgate.

Genkai-Kato, M. (2007). Regime shifts: catastrophic responses of ecosystems to human impacts. Ecological Research, 22(2), 214-219.

Gibson, C., Ostrom, E., \& Ahn, T. (2000). The concept of scale and the human dimensions of global change: a survey. Ecological Economics, 32(2), 217-239.

Grimm, N., G., M., Pickett, S. T. A., \& Redman, C. (2000). Integrated approaches to longterm studies of urban ecological systems. Bioscience, 50(7), 571-584.

Gunderson, L. H., Garmestani, A. S., \& Allen, C. R. (2009). Panarchy: Discontinuities Reveal Similarities in the Dynamic System Structure of Ecological and Social Systems. Ecology and Society, 14(1), 12. Retrieved from http://www.ecologyandsociety.org/vol14/iss1/art15/

Gunderson, L., H. (2000). Ecological resilience in theory and application. Annual Review of Ecology and Systematics, 31, 425-439.

Gunderson, L. H., Allen, C. R., \& Holling, C. S. (Eds.). (2009). Foundations of ecological resilience. Washington: Island Press.

Gunderson, L. H., \& Holling, C. S. (2002). Panarchy: understanding transformations in human and natural systems. Washington, DC: Island Press.

Haigh, R., \& Amaratunga, D. (2010). An integrative review of the built environment discipline's role in the development of society's resilience to disasters. International journal of disaster resilience in the built environment, 1(1), 11-24

Hall, M., Frank, E., Holmes, G., Pfahringer, B., Reutemann, P., \& Witten, I. H. (2009). The Weka data mining software: an update. SIGKDD Explorations, 11(1) 10-18.

Healey, P. (2007). Urban complexity and spatial strategies: towards a relational planning for our times. London: Routledge.

Hexter, J. H. (1979). On historians: reappraisals of some of the makers of modern history. Cambridge, Mass.: Harvard University Press.

Higueras, E. (2006). Urbanismo bioclimatico (Vol. 1). Barcelona: Gustavo Gili. 
Hodgson, T. E. R. (1990). Colonial capital: Wellington, 1865 -1910 Auckland, N.Z.: Random Century.

Holland, A. S. B. (1980). Complex function theory. New York: Elsevier North Holland.

Holland, J. H. (1992). Adaptation in natural and artificial systems: an introductory analysis with applications to biology, control, and artificial intelligence (1st MIT Press ed.). Cambridge, Mass.: MIT Press.

Holling, C. S. (1973). Resilience and Stability of Ecological Systems. Annual Review of Ecology and Systematics, 4(1), 1-23.

Holling, C. S. (1987). Simplifying the complex: The paradigms of ecological function and structure. European Journal of Operational Research, 30(2), 139-146.

Holling, C. S. (1992). Cross-Scale Morphology, Geometry, and Dynamics of Ecosystems. Ecological Monographs, 62(4), 447-502.

Holling, C. S. (2004). From complex regions to complex worlds. Ecology and Society, 9(1), 10. Retrieved from http://www.ecologyandsociety.org/vol9/iss1/art11/

Holling, C. S., \& Goldberg, M. A. (1971). Ecology and Planning. Journal of the American Institute of Planners, 37(4), 221-230.

Holling, C. S., Walker, B., Carpenter, S. R., \& Kinzig, A. (2004). Resilience, adaptability and transformability in social-ecological systems. Ecology and Society, 9(2), 10. Retrieved from http://www.ecologyandsociety.org/vol9/iss2/art5/

Hornborg, A. (2009). Zero-Sum World: Challenges in Conceptualizing Environmental Load Displacement and Ecologically Unequal Exchange in the World-System. International Journal of Comparative Sociology, 50(3-4), 237-262.

Hornik, K. (2012). The R FAQ, from http://CRAN.R-project.org/doc/FAQ/R-FAQ.html

Jerneck, A., \& Olsson, L. (2008). Adaptation and the poor: development, resilience and transition. Climate Policy, 8(2), 170-182.

Jinnai, H. (1995). Tokyo, a spatial anthropology. Berkeley: University of California Press.

Kauffman, S. (1993). The origins of order: self-organization and selection in evolution. New York: Oxford University Press.

King, M. (2003). The Penguin history of New Zealand. Auckland, N.Z.: Penguin Books.

Kitayama, K., Tsukamoto, Y., \& Nishizawa, R. (2010). Tokyo metabolizing [Tokyo metaboraijingu]. Tokyo, Japan: TOTO Pub.

Klein, R. J. T., Nicholls, R. J., \& Thomalla, F. (2003). Resilience to natural hazards: How useful is this concept? Global Environmental Change Part B: Environmental Hazards, 5(1-2), 35-45.

Kropf, K. (1993). The definiton of built form in urban morphology. Doctor of Philosophy, University of Birmingham, Birmingham. 
Krugman, P. (1996). The self-organizing economy. Cambridge, Mass., USA: Blackwell Publishers.

Larkham, P. J., \& Slater, T. R. (1995). After Alnwick: Studying Urban Form in the 1990s. Area, 27(2), 185-186.

Leal del Castillo, G. (2004). Introduccion al ecourbanismo. Bogota: Ecoe ediciones.

Lévesque, L. (2009). Towards an interstitial approach to urban landscape. Territorio (48), 77-82.

Levin, S. A. (1998). Ecosystems and the Biosphere as Complex Adaptive Systems. Ecosystems, 1(5), 431-436.

Levin, S. A. (2005). Self-organization and the emergence of complexity in ecological systems. BioScience, 55(12), 1075-1079.

Lewin, R. (1999). Complexity: life at the edge of chaos (2nd ed.). Chicago, Ill: University of Chicago Press.

Luccarelli, M. (1995). Lewis Mumford and the ecological region: the politics of planning. New York: Guilford Press.

map, E. C. d. c. (Cartographer). (1990). Edo- Tokyo shigai chizu shuusei 1887 nen -1959 nen [ Historical maps of Edo to Tokyo from 1887-1959].

McClain, J. L., Merriman, J. M., \& Ugawa, K. (1994). Edo and Paris: urban life and the state in the early modern era. Ithaca: Cornell University Press.

McGlade, J., McIntosh, B. S., \& Jeffrey, P. (2008). Landscape Sensitivity, Resilience and Sustainable Watershed Management. In P. Koundouri (Ed.), Coping with Water Deficiency (pp. 113-134): Springer.

McLauchlan, G. (2005). A short short history of New Zealand. Auckland, N.Z.: Penguin.

McLauchlan, G. (2008). The life and times of Auckland: the colourful story of a city. Rosedale, N.Z.: Penguin Books.

Milizia, F., \& Cipriani, G. (1785). Principi di architettura civile. Bassano: A spese Remondini di Venezia.

Millennium Ecosystem, A. (2005). Ecosystems and human well-being. Washington, D.C: Island Press.

Moisset, I., \& Paris, O. (Eds.). (2005). Hipotesis de paisaje (I+P editorial ed. Vol. 3-4). Cordoba: I+P editorial.

Morin, E. (2001). Seven complex lessons in education for the future. Paris: UNESCO.

Moudon, A. V. (1997). Urban morphology as an emerging interdisciplinary field. Urban morphology, 1(1), 3-10. 
Muminovic, M., Garcia, E., Vale, B., \& Radovic, D. (2012a). The resilience of the identity of shitamachi in Tokyo. Paper presented at the Place making and identity: rethinking urban approahces to built environment, Jakaarta.

Muminovic, M., Garcia, E., Vale, B., \& Radovic, D. (2012b). Resilient assemblages: the complex identity of Nezu in Tokyo. Paper presented at the New Urban Configurations, Delft University of Technology.

Nakagawa, T. (2005). The Japanese house: in space, memory and language (1st English ed.). Tokyo: International House of Japan.

Nakajima, K. (1996). Uneasy rooms, The concept of Space in Modern Japan. World Policy Journal, 13(4), 67-72.

Oliver, W. H. W., B.R. (Ed.). (1981). The Oxford history of New Zealand. Oxford: Wellington; New York: Clarendon Press; Oxford University Press.

Padua, A. (2010). As bases teoricas da historia ambiental. Estudos Avancados, 24(68), 20.

Pesci, R. (2007). Ambitectura. Hacia un tratado de arquitectura ciudad y ambiente (Vol. 1). La Plata, Argentina: Ediciones al margen.

Peterson, G., Allen, C. R., \& Holling, C. S. (1998). Ecological Resilience, Biodiversity, and Scale. Ecosystems, 1(1), 6-18.

Pickett, S. T. A., Burch, W. R., Dalton, S. E., Foresman, T. W., Grove, J. M., \& Rowntree, R. (1997). A conceptual framework for the study of human ecosystems in urban areas. Urban Ecosystems, 1(4), 185-199.

Pickett, S. T. A., Cadenasso, M. L., Grove, J., Nilon, C., Pouyat, R., Zipperer, W., \& Costanza, R. (2001). Urban ecological systems: linking terrestrial ecological, physical, and socioeconomic components of metropolitan areas. Annual Review of Ecology and Systematics, 32, 127-157.

Pimm, S. L. (1984). The complexity and stability of ecosystems. Nature, 307(5949), 321326.

Radovic, D. (2008). Another Tokyo. Tokyo: University of Tokyo cSUR and ichii Shobou.

Rappaport, R. (1977). Maladaptation in social systems. In J. Friedman \& J. Rowlands (Eds.), The evolution of social systems: proceedings of a meeting of the Research Seminar in Archaeology and Related Subjects held at the Institute of Archaeology, London University (pp. 49-71). Chicago: Duckworth.

Redman, C., \& Kinzig, A. P. (2003). Resilience of past landscapes: resilience theory, society, and the longue durée. Conservation ecology, 7(1), 14.

Resiliance Alliance (2007a). Assessing and managing resilience in social-ecological systems: A practitioners workbook. Volume 1, version 1.0. Retrieved from http://www.resalliance.org/3871.php

Resiliance Alliance (2007b). Assessing and managing resilience in social-ecological systems: A practitioners workbook. Supplementary notes to the practitioners 
workbook, Volume 2, version 1. Retrieved from http://www.resalliance.org/3871.php

Sarquis, J. (2003a). Itinerarios del proyecto: Ficcion de lo real (Vol. 2). Buenos Aires: Nobuko.

Sarquis, J. (2003b). Itinerarios del proyecto: Ficcion epistemologica (Vol. 1). Buenos Aires: Nobuko.

Schumpeter, J. A. (1994). Capitalism, Socialism and Democracy. London: Taylor \& Francis.

Section, A. C. D. (1969). Auckland's historical background: its relation to central city planning. Auckland: Auckland City Council, Dept. of Works and Services.

Seidensticker, E. (1983). Low city, high city: Tokyo from Edo to the earthquake (1st ed.). New York: Knopf.

Shelton, B. (1999). Learning from the Japanese city: West meets East in urban design. London; New York: E \& FN Spon.

Slater, T. R. (Ed.). (1990). The Built form of western cities: essays for M.R.G. Conzen on the occasion of his 80th birthday Leicester. New York: Leicester University Press.

Solà-Morales i Rubió, M. (1995). Terrain vagues. In C. Davidson (Ed.), Anyplace (Vol. 1, pp. 271). New York: London: MIT Press.

Sorensen, A. (2002). The making of urban Japan: cities and planning from Edo to the twenty-first century. London; New York: Routledge.

Tainter, J. A. (1988). The collapse of complex societies. Cambridge, Cambridgeshire; New York: Cambridge University Press.

Tainter, J. A. (1995). Sustainability of complex societies. Futures, 27(4), 397-407.

Tainter, J. A. (2000). Problem Solving: Complexity, History, Sustainability. Population \& Environment, 22(1), 3-41.

Turner, N., Davidson-Hunt, I., \& O'Flaherty, M. (2003). Living on the Edge: Ecological and Cultural Edges as Sources of Diversity for Social-Ecological Resilience. Human Ecology, 31(3), 439-461.

Tzoulas, K., Korpela, K., Venn, S., Yli-Pelkonen, V., Kaźmierczak, A., Niemela, J., \& James, P. (2007). Promoting ecosystem and human health in urban areas using Green Infrastructure: A literature review. Landscape and Urban Planning, 81(3), 167178.

Vale, L., \& Campanella, T. (2005). The resilient city: how modern cities recover from disaster. New York: Oxford University Press.

Vale, R., \& Vale, B. (2009). Time to eat the dog: the real guide to sustainable living. London: Thames \& Hudson.

van Lieshout M., Dewulf, N., \& Aarts, A. (2011). Do scale frames matter? Scale frame mismatches in the decision making process about a "mega farm" in a small Dutch 
village. Ecology and Society, 16(1), 28. Retrieved from http://www.ecologyandsociety.org/vol16/iss1/art38/

Walker, B. (2007). Module: (1.5) Specified and General Resilience (Document). Retrieved from http://wiki.resalliance.org/index.php/1.5_Specified_and_General_Resilience

Walker, B., \& Meyers, J. A. (2004). Thresholds in ecological and social-ecological systems: a developing database. Ecology and Society, 9(2) 16. Retrieved from http://www.ecologyandsociety.org/vol9/iss2/art3

Walker, B., \& Salt, D. (2006). Resilience thinking:sustaining ecosystems and people in a changing world. Washington, DC: Island Press.

Walker, L. R. (1999). Ecosystems of disturbed ground (1st ed.). Amsterdam; New York: Elsevier.

Wegener, M., Gnad, F., \& Vannahme, M. (1986). The time scale of urban change. In B. Hutchinson \& M. Batty (Eds.), Advances in Urban System Modelling (pp. 175-197). Amsterdam: North Holland.

Whitehand, J. W. R. (2007). Conzenian urban morphology and urban landscapes. Paper presented at the Proceedings, 6th International Space Syntax Symposium, Istanbul.

Whitehand, J. W. R. (1967). Fringe Belts: A Neglected Aspect of Urban Geography. Transactions of the Institute of British Geographers(41), 223-233.

Whitehand, J. W. R. (1977). The Basis for an Historico-Geographical Theory of Urban Form. Transactions of the Institute of British Geographers, 2(3), 400-416.

Whitehand, J. W. R. (1981). Background to the urban morphogenetic tradition. In W. J. W. R. (Ed.), The Urban landscape: historical development and management. Papers by M.R.G. Conzen (Vol. 13, pp. vii, 166). London: New York: Academic Press.

Witten, I. H., Frank, E., \& Hall, M. A. (2011). Data mining: practical machine learning tools and techniques (3rd ed.). Burlington, MA: Morgan Kaufmann.

Wu, J., \& Li, H. (2006). Concepts of scale and scaling. In J. Wu, K. B. Jones, H. Li \& O. L. Loucks (Eds.), Scaling and uncertainty analysis in ecology: methods and applications. (pp. 13). Dordrecht: Springer. 
9. Appendices 


\section{Appendix 1: Timeline for Auckland (chapter 4)}

\begin{tabular}{|c|c|c|c|}
\hline Period & Structuring Processes & Structuring variables & Morphological Change \\
\hline \multirow{3}{*}{$\begin{array}{l}\text { 1840-1859: } \\
\text { Inaugural years } \\
\text { Population } \\
2,895 \\
\text { Built area } \\
33.2 \text { ha }\end{array}$} & $\begin{array}{l}\text { 1840.Founded as New } \\
\text { Zealand's capital. }\end{array}$ & $\begin{array}{l}\text { 1841. Mathew's Original } \\
\text { plan }\end{array}$ & $\begin{array}{l}\text { Grid Pattern. } \\
\text { 1841. British fort on Point } \\
\text { Britomart. }\end{array}$ \\
\hline & $\begin{array}{l}\text { Township around } \\
\text { Commercial Bay }\end{array}$ & $\begin{array}{l}\text { Settlers around } \\
\text { Commercial Bay }\end{array}$ & $\begin{array}{l}\text { Queen Street and Shortland } \\
\text { Street. }\end{array}$ \\
\hline & $\begin{array}{l}\text { Freemans Bay and } \\
\text { Mechanics Bay }\end{array}$ & & $\begin{array}{l}\text { 1859. Land reclamation. From } \\
\text { Shortland Street to Franklin } \\
\text { Road. }\end{array}$ \\
\hline \multirow{4}{*}{$\begin{array}{l}\text { 1860-1879: Land } \\
\text { wars and } \\
\text { development of rail } \\
\text { lines. } \\
\text { Population } \\
\text { 12,423 (1864) } \\
\text { Built area } \\
565 \text { ha (1871) }\end{array}$} & $\begin{array}{l}\text { 1860. Waikato Land } \\
\text { wars }\end{array}$ & $\begin{array}{l}\text { Enlargement of city's } \\
\text { garrison }\end{array}$ & $\begin{array}{l}\text { Construction of Great South } \\
\text { Road and a chain of military } \\
\text { redoubts }\end{array}$ \\
\hline & $\begin{array}{l}\text { 1864.Development of } \\
\text { the southern region } \\
\text { after war. }\end{array}$ & $\begin{array}{l}\text { Electric telegraph, regular } \\
\text { horse bus }\end{array}$ & \multirow[t]{3}{*}{$\begin{array}{l}\text { Satellite townships. Settlement } \\
\text { continued south towards Mt } \\
\text { Eden, Otahuhu and Panmure. }\end{array}$} \\
\hline & $\begin{array}{l}\text { 1865. Loss of capital } \\
\text { status. Economic } \\
\text { crisis. }\end{array}$ & $\begin{array}{l}\text { A cottage and an acre of } \\
\text { land per soldier. }\end{array}$ & \\
\hline & $\begin{array}{l}\text { 1870. Discovery of } \\
\text { gold at Thames. }\end{array}$ & $\begin{array}{l}\text { 1869. Piped water to town. } \\
\text { 1870. Railway links }\end{array}$ & \\
\hline \multirow{3}{*}{$\begin{array}{l}\text { 1880-1899: } \\
\text { Economic expansion } \\
\text { Population } \\
57,616(1896)\end{array}$} & $\begin{array}{l}\text { Growth in commercial } \\
\text { services. } \\
\text { Large manufacturing. }\end{array}$ & $\begin{array}{l}\text { Working class settlements } \\
\text { on the town fringes around } \\
\text { industries and railway }\end{array}$ & $\begin{array}{l}\text { Residential growth in } \\
\text { Birkenhead and New Lynn. } \\
\text { Workshops in New Market. }\end{array}$ \\
\hline & \multirow[t]{2}{*}{$\begin{array}{l}\text { Development of } \\
\text { public transport. }\end{array}$} & Rail links & $\begin{array}{l}\text { Settlements beside the line at } \\
\text { Henderson, New Lynn and } \\
\text { Glen Eden. }\end{array}$ \\
\hline & & 1881. Ferries & $\begin{array}{l}\text { Suburban growth in } \\
\text { Devonport, Takapuna, } \\
\text { Northcote and Birkenhead. }\end{array}$ \\
\hline \multirow{2}{*}{$\begin{array}{l}\text { 1900-1929: Turning } \\
\text { into a city } \\
\text { Population } \\
\text { 133,712 (1916) } \\
\text { Built area } \\
5039 \text { ha }\end{array}$} & Dairy farming & $\begin{array}{l}\text { Dairying expanded } \\
\text { throughout the periphery. }\end{array}$ & $\begin{array}{l}\text { Urban form change } \\
\text { dramatically. }\end{array}$ \\
\hline & $\begin{array}{l}\text { Middle-class families } \\
\text { left inner city }\end{array}$ & $\begin{array}{l}\text { Electric tramway, } \\
\text { motorcars and buses } \\
\text { started to emerge. }\end{array}$ & $\begin{array}{l}\text { Growth of Epsom, Remuera } \\
\text { and the North Shore. Middle } \\
\text { class went to Mount Albert. }\end{array}$ \\
\hline \multirow[t]{2}{*}{$\begin{array}{l}\text { 1930-1949: } \\
\text { Emergence of State } \\
\text { housing provision } \\
\text { Population } \\
251,667(1945) \\
\text { Built area 13,642 ha } \\
(1945)\end{array}$} & $\begin{array}{l}\text { Depression of 1930s } \\
\text { slowed down } \\
\text { population growth } \\
\text { and reduced privates } \\
\text { initiatives }\end{array}$ & $\begin{array}{l}\text { State intervention in the } \\
\text { provisioning of housing. } \\
\text { Car ownership made } \\
\text { accessibility easier to go in } \\
\text { and out of Auckland. } \\
\text { Completion of Tamaki } \\
\text { Drive }\end{array}$ & $\begin{array}{l}\text { Single units suburban homes } \\
\text { for families. } \\
\text { Building of inner-city } \\
\text { apartment blocks in the 1940s. } \\
\text { Flats on Symonds Street and } \\
\text { Greys Avenue. }\end{array}$ \\
\hline & $\begin{array}{l}\text { Renewal and growth. } \\
\text { Restrictions on } \\
\text { imports. }\end{array}$ & $\begin{array}{l}\text { Local manufacturing } \\
\text { Development. } \\
\text { Hydroelectric stations } \\
\text { along Waikato river. } \\
\end{array}$ & $\begin{array}{l}\text { Industrial area around } \\
\text { Penrose, Otahuhu and Mt } \\
\text { Wellington. }\end{array}$ \\
\hline \multirow{2}{*}{$\begin{array}{l}\text { 1950-1969 Major } \\
\text { decisions } \\
\text { Population } \\
\text { 535,167 (1966) } \\
\text { Built area 26,793 ha }\end{array}$} & $\begin{array}{l}\text { Regional planning. } \\
\text { Development of } \\
\text { motorway system. }\end{array}$ & $\begin{array}{l}\text { The 'Master } \\
\text { Transportation Plan' for } \\
\text { Metropolitan Auckland } \\
\text { (1955) }\end{array}$ & $\begin{array}{l}\text { Disperse city. Significant } \\
\text { suburban development } \\
\text { Detached houses on large lots. } \\
\text { Decline of central city. }\end{array}$ \\
\hline & & Harbour Bridge in 1959. & $\begin{array}{l}\text { New suburbs emerged on the } \\
\text { North Shore }\end{array}$ \\
\hline
\end{tabular}




\begin{tabular}{|c|c|c|c|}
\hline (1964) & $\begin{array}{l}\text { Plans to consolidate } \\
\text { urban growth. }\end{array}$ & $\begin{array}{l}\text { 1961. Regional } \\
\text { Development Plan } \\
\text { 1967. Regional Master } \\
\text { Plan }\end{array}$ & \\
\hline \multirow{2}{*}{$\begin{array}{l}\text { 1970-1979: } \\
\text { Continued outward } \\
\text { growth } \\
\text { Population } \\
707,607(1976) \\
\text { Built area } 37,000 \text { ha } \\
(1975)\end{array}$} & $\begin{array}{l}\text { Further development } \\
\text { of Motorway system. }\end{array}$ & $\begin{array}{l}\text { Work on Central Junction. } \\
\text { State housing }\end{array}$ & $\begin{array}{l}\text { New housing developments in } \\
\text { East Tamaki, also experienced } \\
\text { considerable growth }\end{array}$ \\
\hline & $\begin{array}{l}\text { Growing awareness } \\
\text { about urban sprawl. }\end{array}$ & $\begin{array}{l}\text { 1974. Regional Planning } \\
\text { Scheme for Auckland. } \\
\text { 1975. Alternatives for } \\
\text { Future Regional Growth. }\end{array}$ & \\
\hline \multirow[t]{2}{*}{$\begin{array}{l}\text { 1980-1989: } \\
\text { Intensification } \\
\text { through infill } \\
\text { housing. } \\
\text { Population } \\
754,845(1986) \\
\text { Built area 40,022 ha } \\
\text { (1987) }\end{array}$} & $\begin{array}{l}\text { Broad economic } \\
\text { deregulation, changes } \\
\text { to the welfare system, } \\
\text { local government } \\
\text { reform, recession due } \\
\text { to market crash of } \\
\text { 1987, and population } \\
\text { growth. }\end{array}$ & $\begin{array}{l}\text { Growth in housing } \\
\text { emerged from infill as well } \\
\text { as the development of } \\
\text { available land. }\end{array}$ & $\begin{array}{l}\text { Dominance of flats in } \\
\text { Remuera, Kohimarama, } \\
\text { Mission Bay and St Heliers } \\
\text { High growth also in the Wiri } \\
\text { area. }\end{array}$ \\
\hline & $\begin{array}{l}\text { Growth for industries } \\
\text { located in the CBD, } \\
\text { like insurance and } \\
\text { finance. Changing } \\
\text { lifestyle patterns and } \\
\text { household } \\
\text { composition }\end{array}$ & $\begin{array}{l}\text { Demand for inner city } \\
\text { apartments }\end{array}$ & $\begin{array}{l}\text { Many converted their office } \\
\text { blocks into apartments, and } \\
\text { empty lots where office } \\
\text { buildings were planned } \\
\text { became open-air car parks }\end{array}$ \\
\hline \multirow[t]{2}{*}{$\begin{array}{l}\text { 1990-1999: } \\
\text { Strategies for growth } \\
\text { Population } \\
997,940(1996) \\
\text { Built area } \\
?\end{array}$} & $\begin{array}{l}\text { Change in national } \\
\text { immigration policy } \\
\text { that allowed new } \\
\text { migrants to enter } \\
\text { New Zealand }\end{array}$ & $\begin{array}{l}\text { While the outer suburbs } \\
\text { absorbed a large } \\
\text { proportion of growth in } \\
\text { the 1990s, the inner areas } \\
\text { and the CBD also } \\
\text { accommodated a growing } \\
\text { number of new residential } \\
\text { developments. }\end{array}$ & $\begin{array}{l}\text { Continuation of expansion } \\
\text { around urban edge } \\
\text { particularly in south east at } \\
\text { Dannemora, Botany Downs } \\
\text { and East Tamaki } \\
\text { Revitalization of the Central } \\
\text { Business District }\end{array}$ \\
\hline & $\begin{array}{l}\text { Pressures intensified } \\
\text { on transport, housing } \\
\text { and infrastructure }\end{array}$ & $\begin{array}{l}\text { Regional Growth Forum } \\
\text { created, development of } \\
\text { the Regional Growth } \\
\text { Strategy and delineation of } \\
\text { Metropolitan Urban Limit. }\end{array}$ & $\begin{array}{l}\text { Compact urban form with } \\
\text { growth within existing } \\
\text { metropolitan area. Varieties of } \\
\text { housing and mixed use } \\
\text { activities. Limited expansion } \\
\text { on green areas. Protection of } \\
\text { the coast. }\end{array}$ \\
\hline \multirow[t]{2}{*}{ 2000-present } & $\begin{array}{l}1.2 \text { million people } \\
\text { with a } 90 \% \text { living in } \\
\text { urban areas. }\end{array}$ & $\begin{array}{l}\text { Significant investment in } \\
\text { public transport } \\
\text { infrastructure. }\end{array}$ & $\begin{array}{l}\text { Intensification through infill } \\
\text { and redevelopment }\end{array}$ \\
\hline & & $\begin{array}{l}\text { Inner city resident } \\
\text { population growth } 500 \% \text {. }\end{array}$ & $\begin{array}{l}\text { Apartment building } \\
\text { development in the CBD. } \\
\text { Low-density development at } \\
\text { the periphery. New suburbs. }\end{array}$ \\
\hline
\end{tabular}


Appendix 2: Maps of blocks in the urban landscape of Auckland CBD

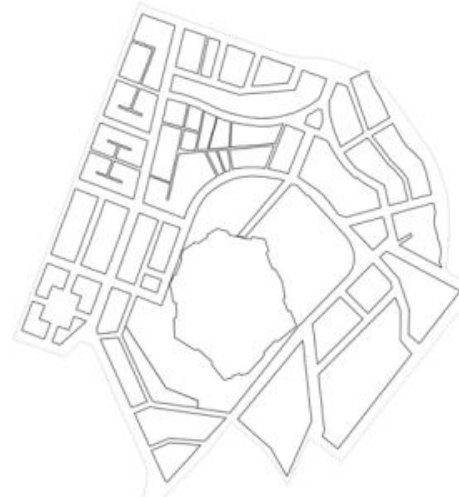

1866

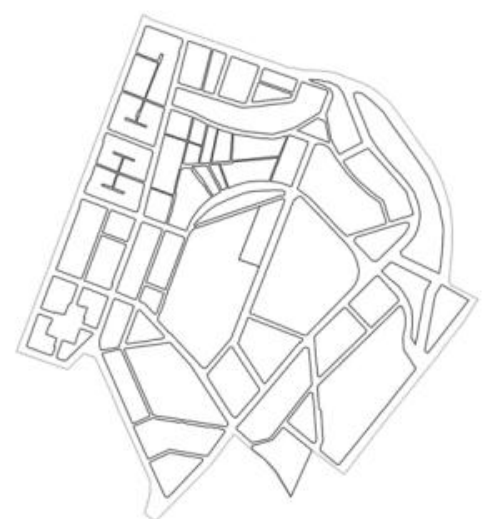

1924

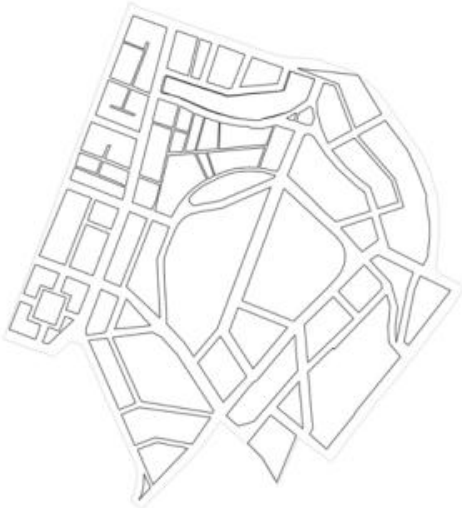

1882

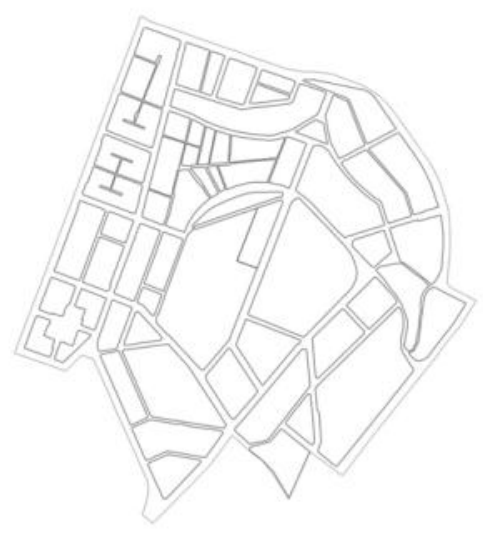

1908
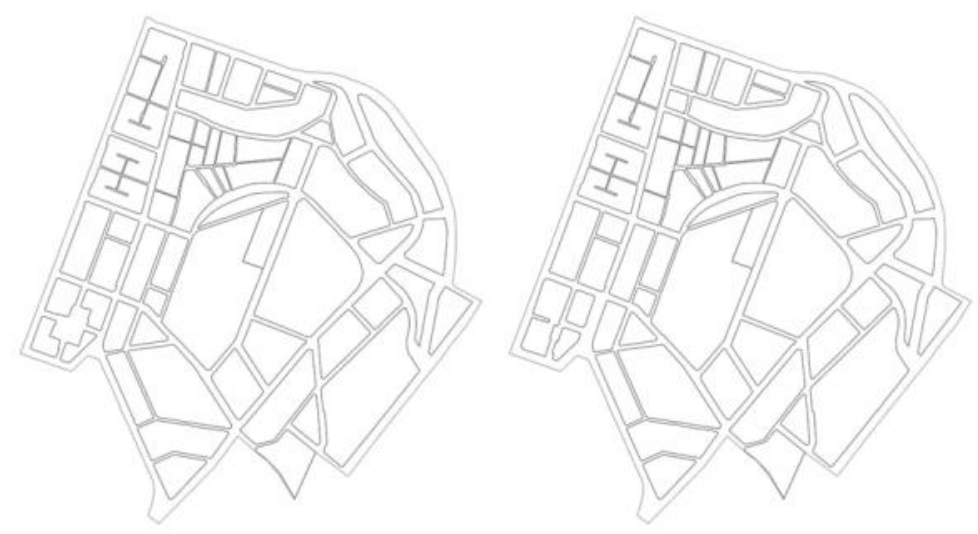

1931

1948
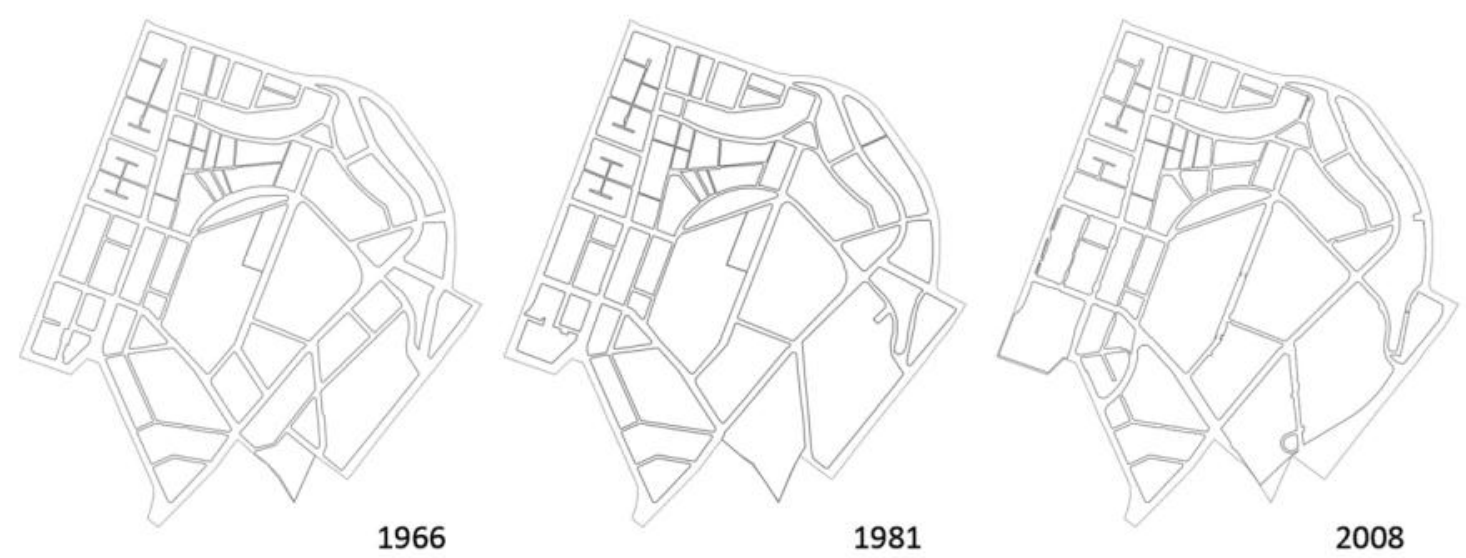
Appendix 3: Maps of plots in the urban landscape of Auckland CBD

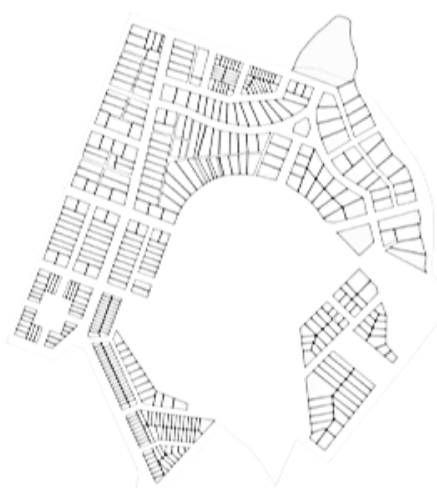

1866

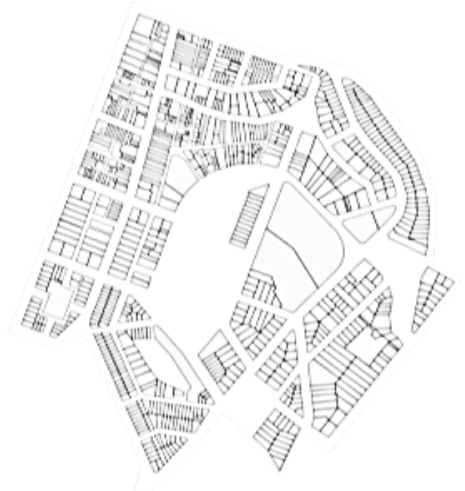

1924

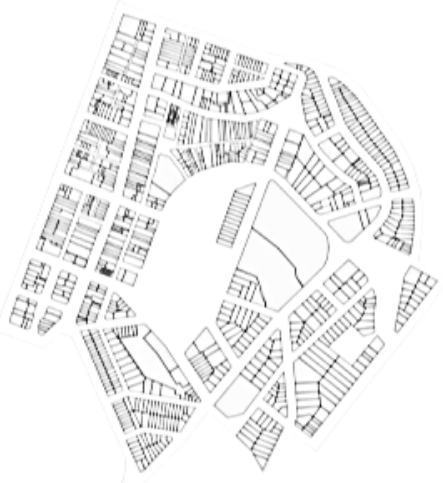

1931
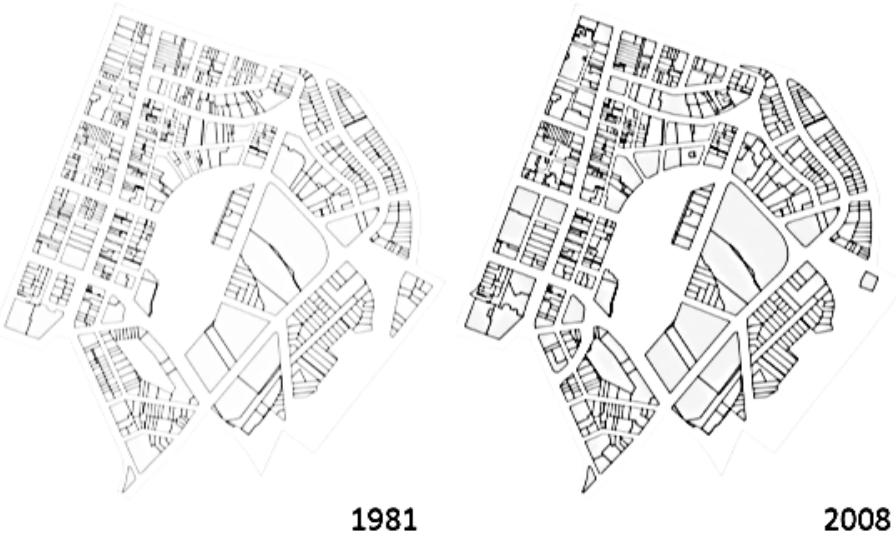


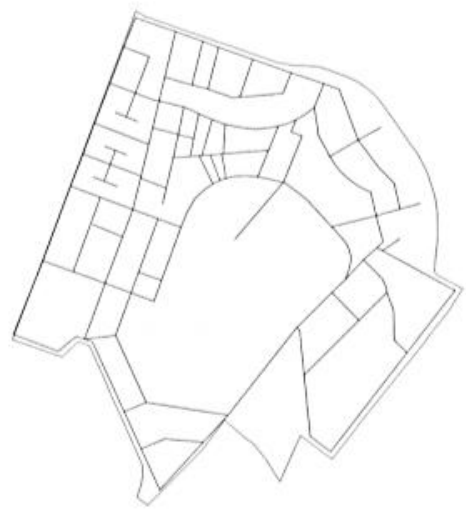

1866

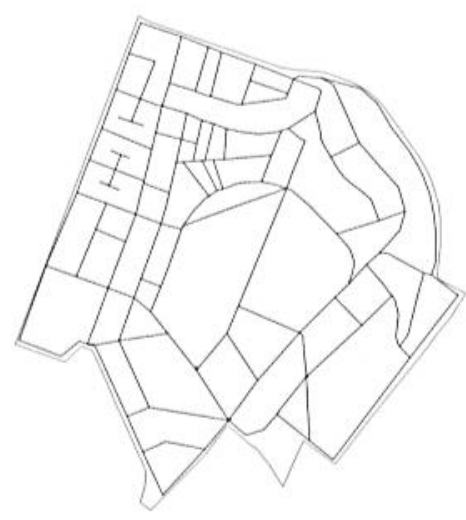

1924

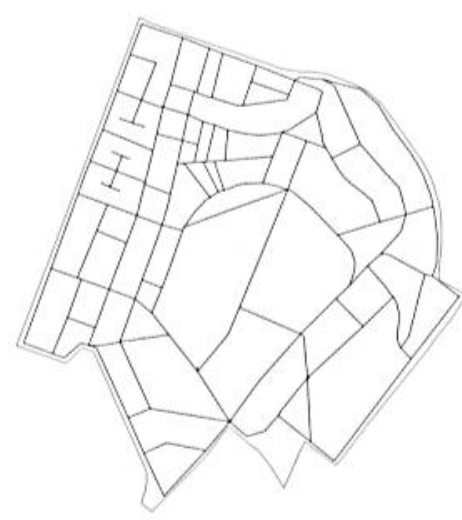

1966

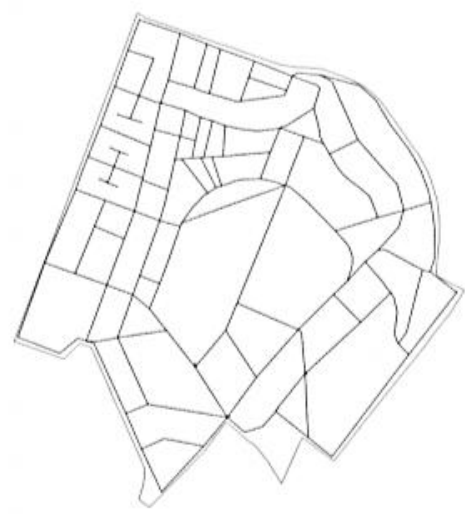

1931

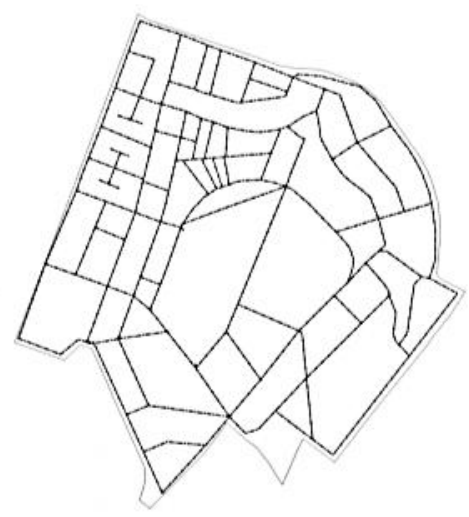

1908

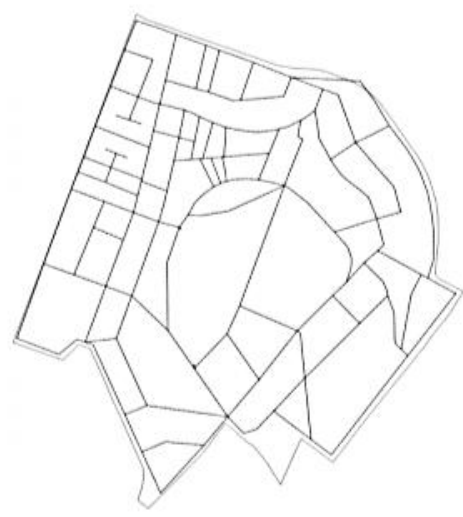

1882

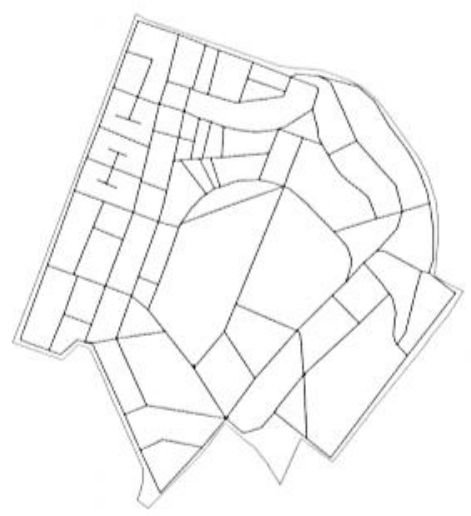

1948

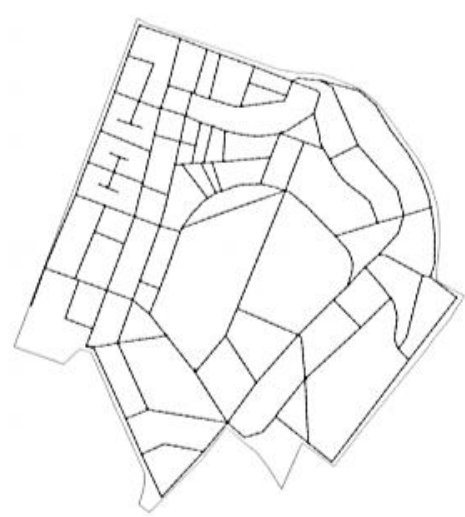

1981

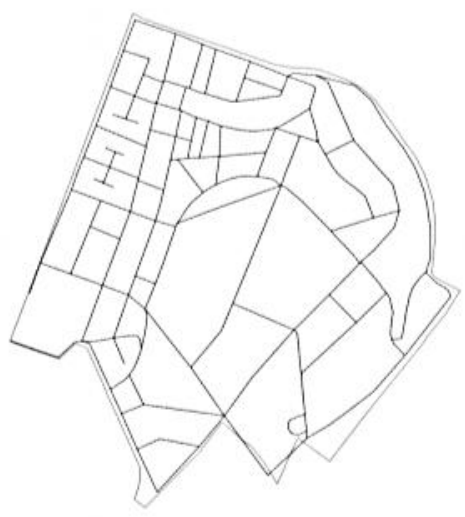

2008 
Appendix 5: Maps of building footprints in Auckland CBD (chapter 4)
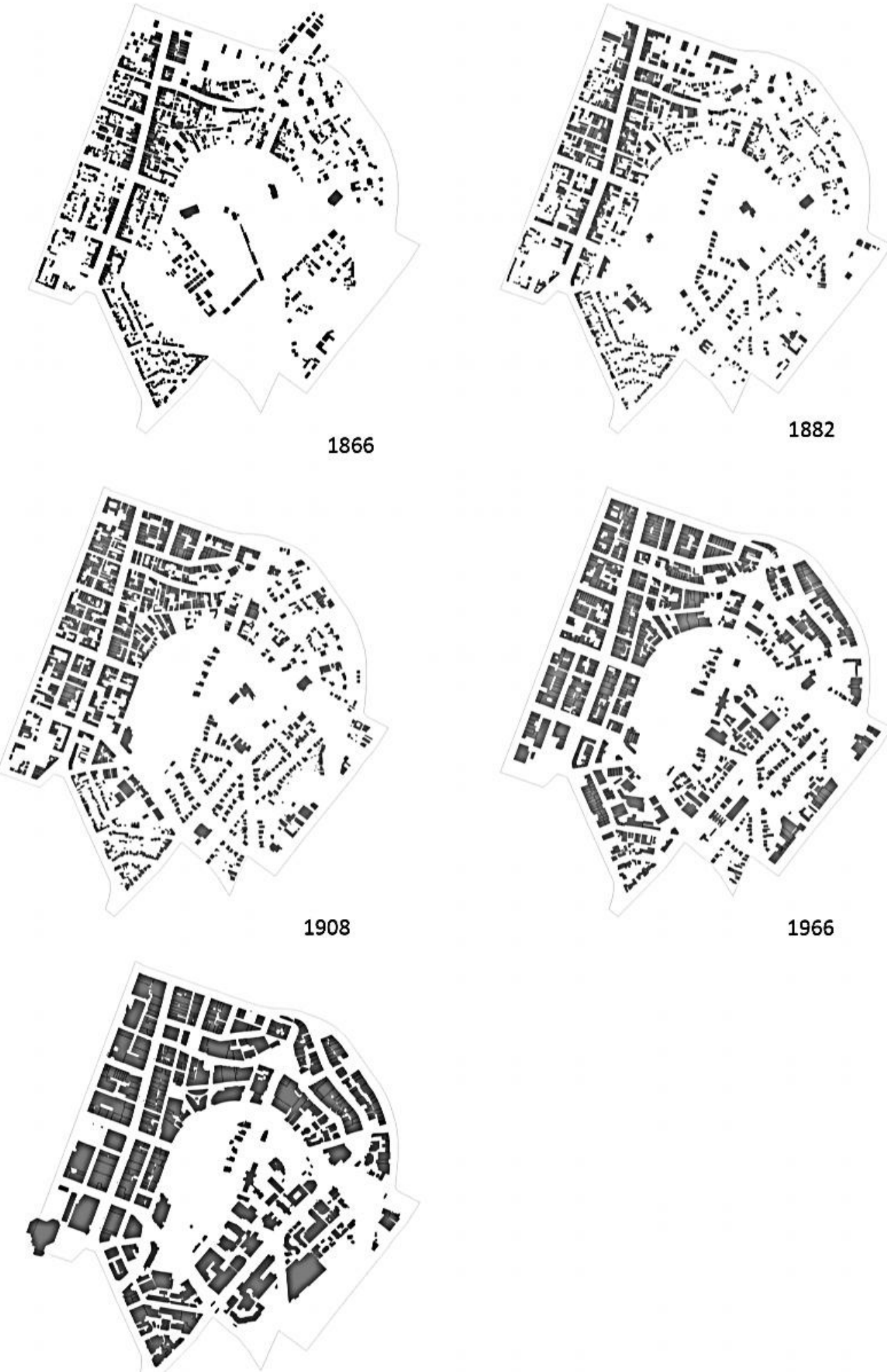

2008 


\section{Appendix 6: areas and number of features per BF, PL, BL, ST (chapter 4)}

\begin{tabular}{|c|c|c|c|c|c|c|c|c|c|c|}
\hline & & 1866 & 1882 & 1908 & 1924 & 1931 & 1948 & 1966 & 1981 & 2008 \\
\hline \multirow{4}{*}{$\begin{array}{l}\text { Building } \\
\text { footprint }\end{array}$} & Mean & 94.45 & 110.12 & 168.71 & 216.49 & 220.32 & 282.71 & 341.50 & 504.91 & 799.42 \\
\hline & StdDev & 100.97 & 126.68 & 169.71 & 212.34 & 215.67 & 269.85 & 324.55 & 634.16 & 1191.07 \\
\hline & Sum & 126757.49 & 151746.00 & 223875.00 & 244650.60 & 246069.57 & 268904.83 & 299495.00 & 326000.31 & 373328.76 \\
\hline & $\mathbf{N}$ & 1342.00 & 1378.00 & 1327.00 & 1450.00 & 1458.00 & 1593.00 & 877.00 & 730.00 & 467.00 \\
\hline \multirow[t]{4}{*}{ Plots } & Mean & 937.15 & 980.39 & 824.00 & 585.85 & 558.50 & 609.01 & 660.84 & 657.02 & 816.19 \\
\hline & StdDev & 1172.35 & 2211.57 & 2033.49 & 1299.76 & 1291.34 & 1318.39 & 1570.18 & 1512.01 & 1727.04 \\
\hline & Sum & 428279.0 & 584311.0 & 581745.0 & 546598.0 & 542866.0 & 564554.0 & 586161.9 & 540729.0 & 542764.0 \\
\hline & $\mathrm{N}$ & 457.00 & 596.00 & 706.00 & 933.00 & 972.00 & 927.00 & 887.00 & 823.00 & 665.00 \\
\hline \multirow[t]{4}{*}{ Blocks } & Mean & 15309.92 & 10963.67 & 12189.68 & 12213.77 & 11994.03 & 12022.36 & 12022.36 & 13610.30 & 14523.50 \\
\hline & StdDev & 31945.23 & 12325.95 & 12762.77 & 13195.31 & 12873.30 & 12825.75 & 12825.75 & 15703.50 & 17414.22 \\
\hline & Sum & 780806.00 & 690711.00 & 755760.00 & 732826.00 & 731636.00 & 733364.00 & 733364.00 & 762176.83 & 784268.84 \\
\hline & $\mathbf{N}$ & 51.00 & 63.00 & 62.00 & 60.00 & 61.00 & 61.00 & 61.00 & 56.00 & 54.00 \\
\hline \multirow[t]{4}{*}{ Streets } & Mean & 254.66 & 279.30 & 267.37 & 272.57 & 267.98 & 276.20 & 276.20 & 264.61 & 254.48 \\
\hline & StdDev & 206.19 & 215.37 & 212.98 & 212.39 & 217.03 & 223.98 & 223.98 & 220.31 & 245.63 \\
\hline & Sum & 14261.00 & 17037.00 & 17379.00 & 17717.00 & 16615.00 & 17677.00 & 17677.00 & 16406.00 & 16032.00 \\
\hline & $\mathrm{N}$ & 56.00 & 61.00 & 65.00 & 65.00 & 62.00 & 64.00 & 64.00 & 62.00 & 63.00 \\
\hline
\end{tabular}


Appendix 7: Key map with name of elements of the built environment (chapter 5)

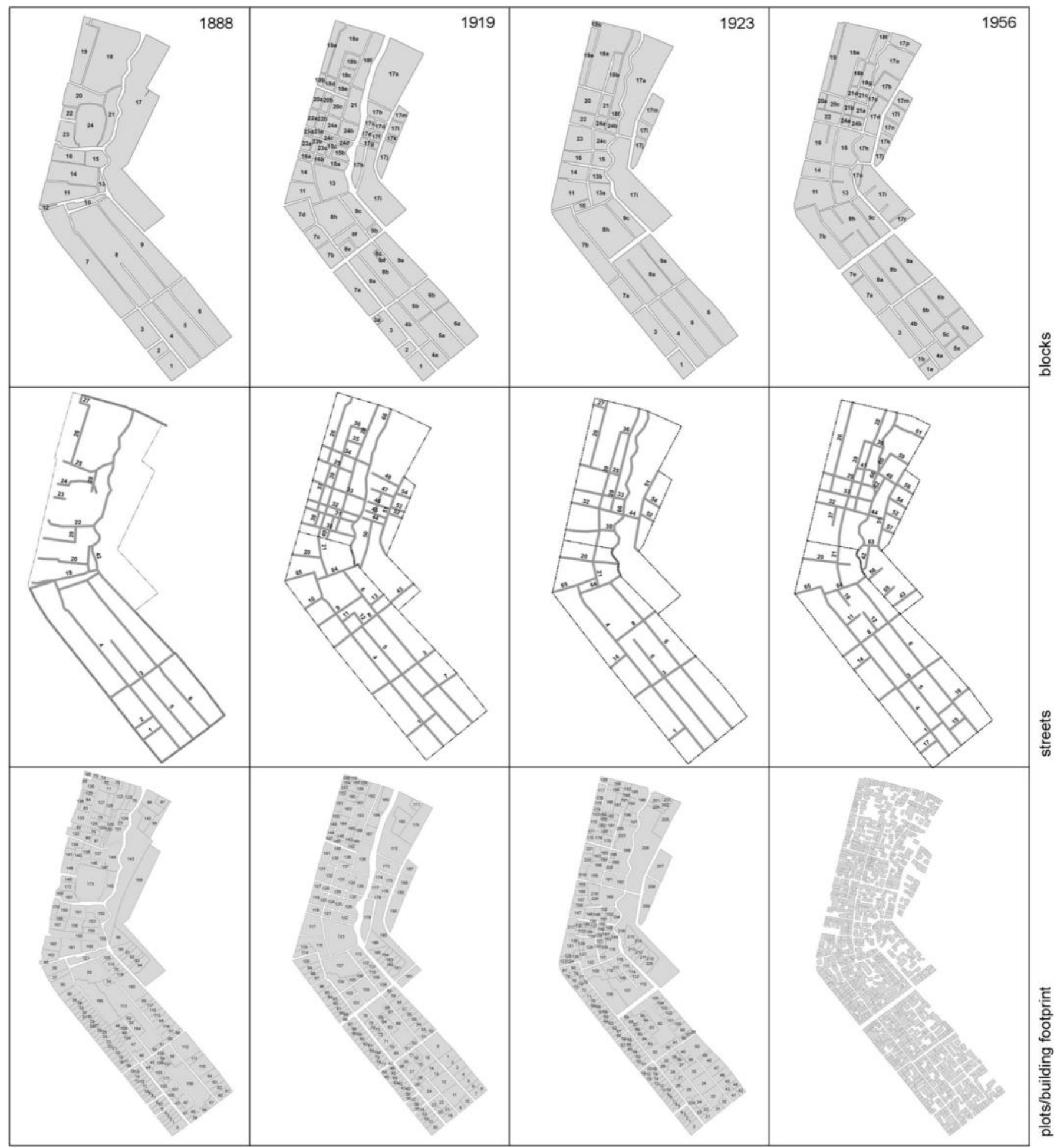

(1) $\longdiv { 1 0 0 \quad 2 0 0 }$ 
1. Appendix 8: Degree of change in blocks in Nezu (chapter 5) 
2. Appendix 9: Degree of change in plots in Nezu (chapter 5) 
3. Appendix 10: Degree of change in streets in Nezu (chapter5) 
4. Appendix 11: Areas and clusters in Nezu 1888 (chapter 5 and 6)
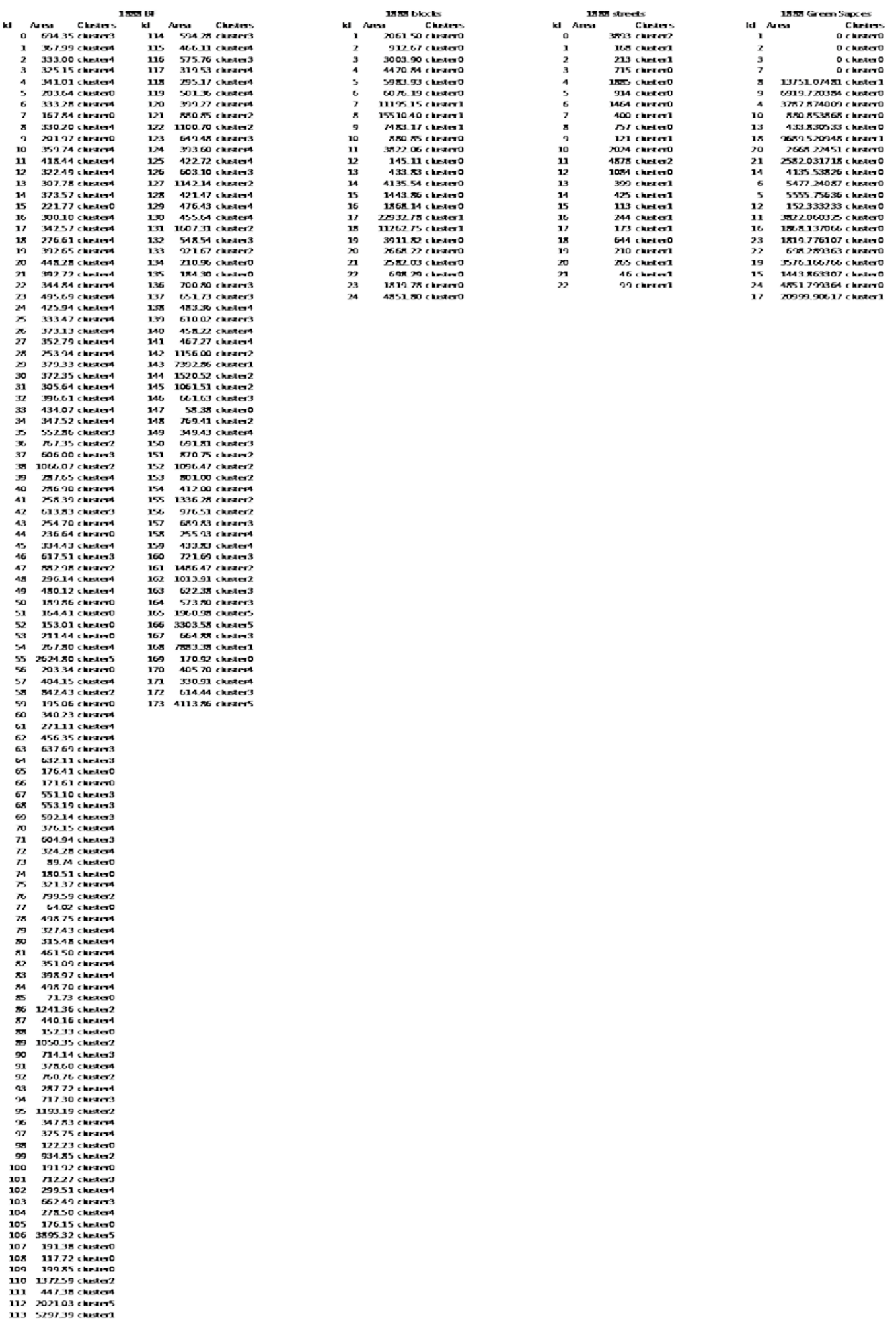


\section{Appendix 12: Areas and clusters in Nezu 1919 (chapter 5 and 6)}
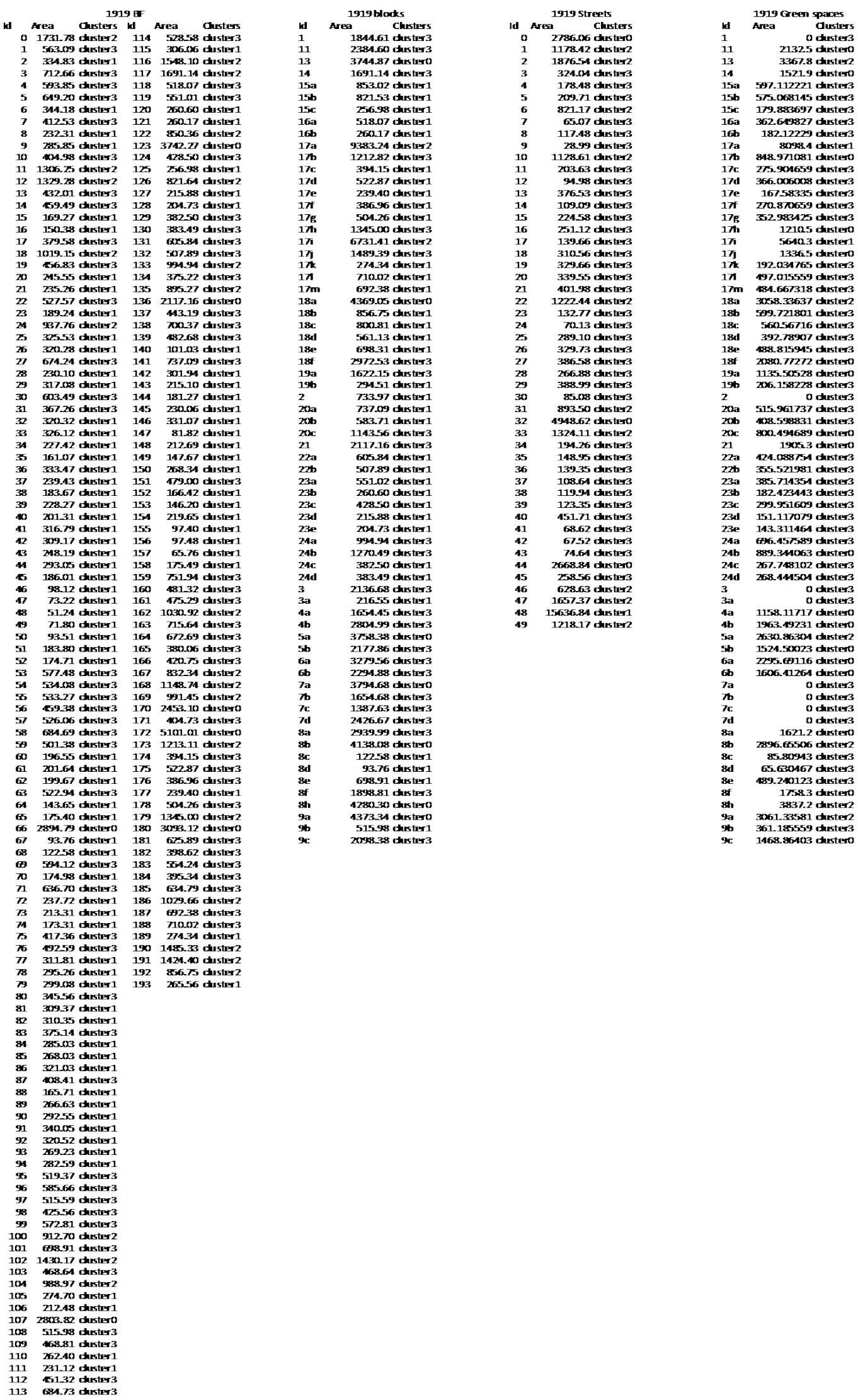
6. Appendix 13: Areas and clusters in Nezu 1932 (chapter 5 and 6)
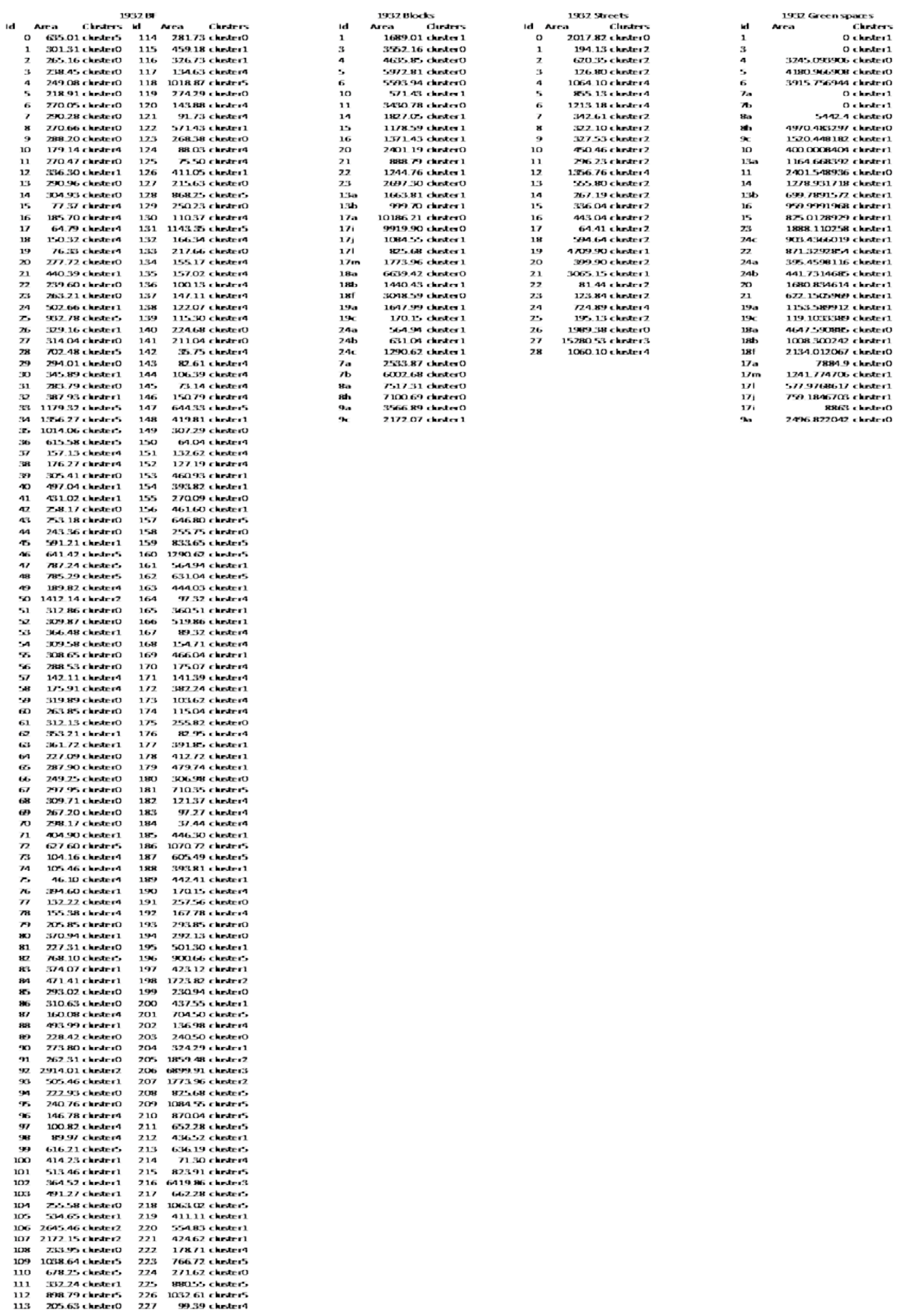


\section{Appendix 14: Areas and clusters in Nezu 1956 (chapter 5 and 6)}

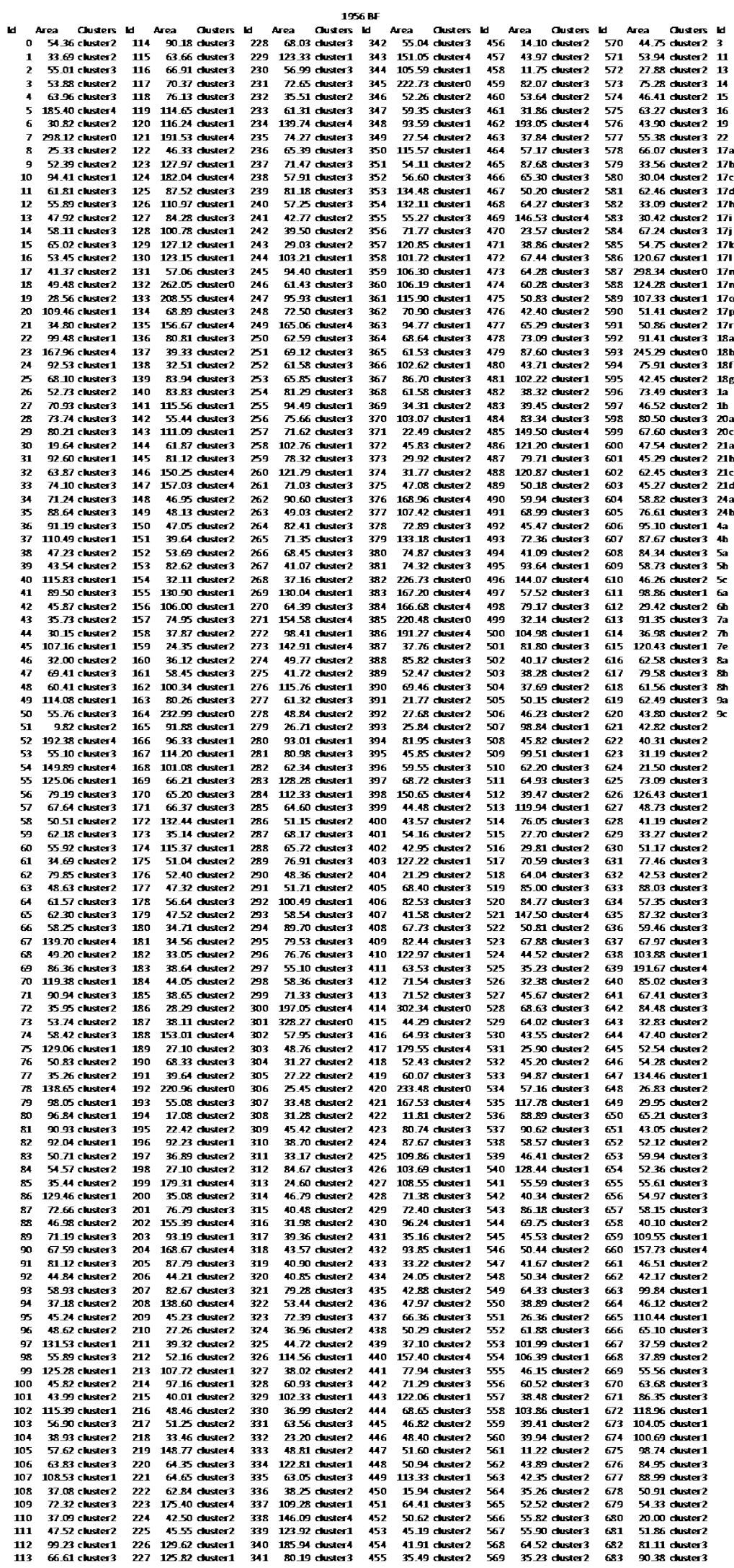

\begin{tabular}{|c|c|c|c|c|c|c|c|}
\hline & & & $95 x$ & & & & \\
\hline & & & & & & & \\
\hline & chustero & & & chusters & & 138.448 & instero \\
\hline & chersoro & 1 & 9.85 & idusters & & 159392621 & instero \\
\hline & chestero & 2 & 113291 & chusters & & $476247476 \mathrm{c}$ & dustero \\
\hline & chestero 0 & & $57.79 \mathrm{c}$ & & & & \\
\hline & chestero & 4 & 305.64 & chustero & & 943.7938730 & destert \\
\hline & chustero & 5 & 9144 & & & $6532165 / 3$ & \\
\hline 19 & chustero & 6 & $10382 \mathrm{c}$ & chuster 3 & & 7763817850 & chustero \\
\hline 182 & custeri & 7 & 114.84 & chuster 3 & & 376.707924 & \\
\hline 6258 & chustero & 8 & 851.01 & dusters & & & chestero \\
\hline 350.35 & chestero & 9 & 7818 & chuster 3 & & & chster1 \\
\hline 39308 & chestert & 10 & 1528.68 & chusters & 11 & 1504.56943 & dustert \\
\hline 193695 & chistero & 11 & 10188 & duster 3 & & & dustert \\
\hline 19225 & chustero & 12 & 7.65 & chuster 3 & & 1400.51973 & chstert \\
\hline 565100 & custero & 13 & 124.65 & chuster 3 & & $59 \mathrm{c}$ & dustert \\
\hline 51099 & chustert & 14 & 264.43 & chustero & & & chstero \\
\hline 69662 & chestert & 15 & 113.49 & chuster 3 & 16 & & chstert \\
\hline 103333 & chester1 & 16 & 20459 & chusterto & & & chuster? \\
\hline 96859 & chestert & 17 & 349.46 & & & & chestero \\
\hline 794.78 & choster1 & 18 & 1226.89 & chusters & & & chestert \\
\hline 61814 & chusteri & 19 & 107.45 & & & & \\
\hline 153719 & dustero & 20 & 607.65 & chuster1 & 21 & & anstero \\
\hline 85757 & chuster1 & 21 & 180.42 & chustero & 22 & & chustert \\
\hline 768846 & chustero & 22 & 367.62 & chustero & 23 & $15 \mathrm{c}$ & chuster1 \\
\hline 790.08 & chuster1 & 23 & 481.01 & chuster1 & 24 & & chestero \\
\hline 125120 & chuster1 & 24 & 637360 & chuster1 & 25 & & chustero \\
\hline 1107.00 & chustert & 25 & 20.55 & Chusterto & 26 & $\cos 3 \mathrm{c}$ & desterto \\
\hline 0.02 & chuster1 & 26 & 493314 & chusterst & 27 & & 1 destero \\
\hline & chuster1 & 27 & 85.82 & chuster 3 & 28 & 335.411089 & chustero \\
\hline 47861 & custern & 28 & 1465100 & custer? & 29 & 102004855 & custero \\
\hline 119085 & & 29 & 1245.87 & & & 267.738415 & chuster 0 \\
\hline $\begin{array}{l}88872 \\
45844\end{array}$ & $\begin{array}{l}\text { chistent } \\
\text { custert }\end{array}$ & $\begin{array}{l}30 \\
31\end{array}$ & $\begin{array}{l}152298 \\
311323\end{array}$ & $\begin{array}{l}\text { cisstens } \\
\text { custert }\end{array}$ & $\begin{array}{l}31 \\
32\end{array}$ & $\begin{array}{r}465555090 \\
316674087\end{array}$ & 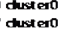 \\
\hline 63229 & choster & 32 & 215.29 & idustero & 33 & 614166805 & instero \\
\hline 40623 & dester & 33 & 50052 & chuster1 & 34 & 528810578 & distero \\
\hline 374.12 & custeri & 34 & 30173 & divstero & 35 & 176685356 & dustert \\
\hline 710 & chsten & 35 & 153.40 & duster 3 & 36 & & dustert \\
\hline 151869 & custeri & 36 & 10881 & chuster 3 & 37 & 1887 & chustert \\
\hline 2080.81 & chustero & 37 & 101.67 & chuster 3 & 38 & 6924 & chustero \\
\hline 126494 & custert & 38 & 13717 & chuster 3 & 39 & & \\
\hline 331785 & cisstero & 39 & 5959 & & 40 & & \\
\hline 1454.45 & ciuster1 & 40 & 108.70 & chuster 3 & 41 & & chestero \\
\hline 281 & chestero & 41 & 113.16 & 3 & 42 & & \\
\hline 316697 & chustero & 42 & 74.87 & chuster 3 & 43 & & dustero \\
\hline 2830.67 & chestero & 43 & 6954 & chster 3 & 44 & & \\
\hline 518159 & chustero & 44 & 230025 & chustero & 45 & 50 & chustero \\
\hline 103631 & Cinstent & 45 & & & 46 & $4 \mathrm{c}$ & \\
\hline 332380 & chustero & & & & 47 & $39 c$ & anstero \\
\hline 409241 & chestero & & & & 48 & 20 & \\
\hline $\begin{array}{l}784.09 \\
26251\end{array}$ & Custero & & & & 49 & $390209142 \mathrm{C}$ & chester? \\
\hline & & & & & & 177.7388120 & \\
\hline & & & & & & & dustero \\
\hline & & & & & 32 & & \\
\hline & & & & & 92 & & \\
\hline & & & & & 93 & $116 \mathrm{c}$ & cherter 3 \\
\hline & & & & & 94 & $967 \mathrm{c}$ & \\
\hline & & & & & 95 & $552 c$ & \\
\hline & & & & & 96 & $853 \mathrm{c}$ & duster 3 \\
\hline & & & & & 97 & $3 \pi$ & chister 3 \\
\hline & & & & & 98 & & \\
\hline & & & & & 99 & & \\
\hline & & & & & 100 & & \\
\hline & & & & & 101 & 241 & an \\
\hline & & & & & 102 & & \\
\hline & & & & & 103 & $0.688561 \mathrm{c}$ & do \\
\hline & & & & & 104 & $0.834204 \mathrm{C}$ & \\
\hline & & & & & 105 & $4152751 \mathrm{c}$ & anster 3 \\
\hline & & & & & 106 & $4.31328 \mathrm{C}$ & chuster 3 \\
\hline & & & & & 107 & $2879675 \mathrm{C}$ & chuster 3 \\
\hline & & & & & 108 & 2168287 & inster 3 \\
\hline & & & & & 109 & $0.835881 \mathrm{C}$ & \\
\hline & & & & & 110 & $0.487383 \mathrm{C}$ & duster 3 \\
\hline & & & & & & & \\
\hline & & & & & & & \\
\hline & & & & & 114 & 1528783 c & distens \\
\hline
\end{tabular}




\section{Appendix 15: BF Areas and clusters in Nezu 2003 (chapter 5 and 6)}

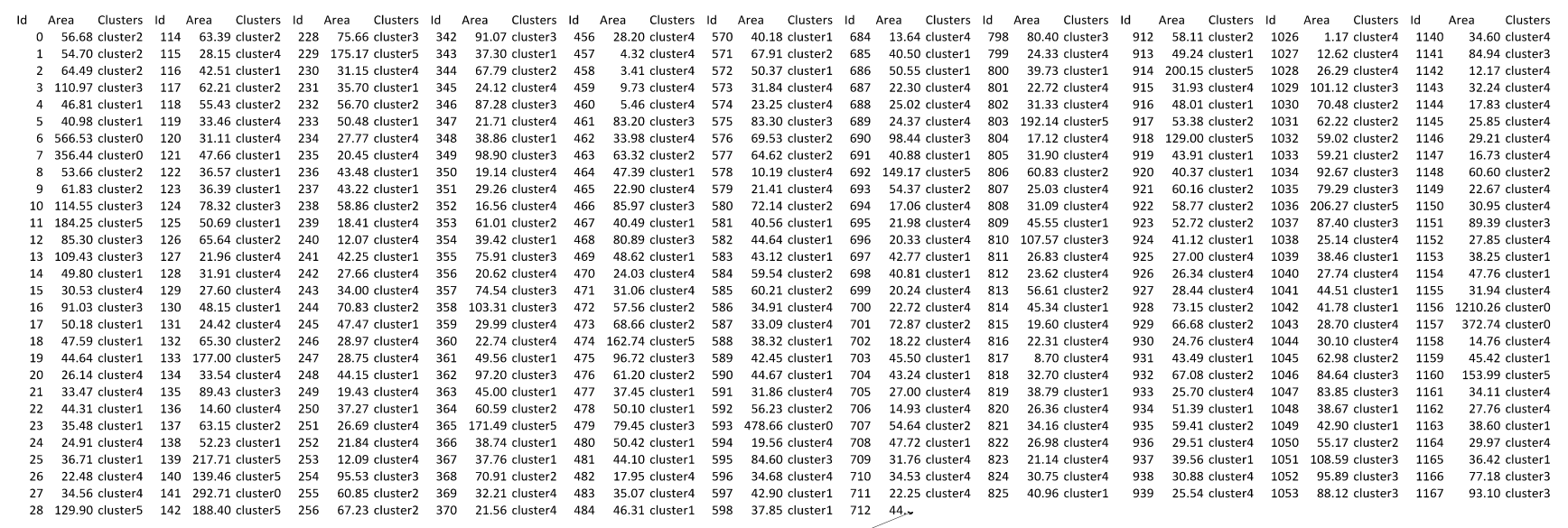


9. Appendix 16: Areas and clusters in Nezu 2003 (chapter 5 and 6)

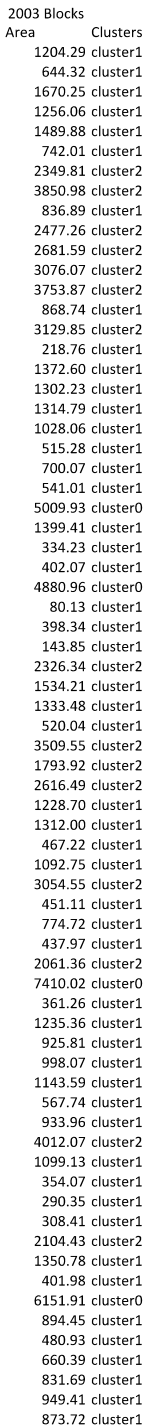

\begin{tabular}{|c|c|}
\hline & \\
\hline & \\
\hline+2 & 175.36 cluster 0 \\
\hline & 77.66 clust \\
\hline 3 & 30.13 cluster0 \\
\hline & 458.73 cluster 0 \\
\hline 3 & 56.17 cluster 0 \\
\hline & 48.67 clustero \\
\hline & 107.58 clustero \\
\hline & 65.09 cluster0 \\
\hline & 42.05 clusterc \\
\hline & 391.11 clusterc \\
\hline & 218.44 cluster \\
\hline 12 & 43.91 cluster \\
\hline 13 & 58.50 cluster \\
\hline 14 & 281.61 clus \\
\hline 15 & 43.04 cluster \\
\hline 16 & 122.15 cluster0 \\
\hline & 82.38 cluster 0 \\
\hline 18 & 116.38 clustero \\
\hline 19 & 92.49 cluster0 \\
\hline 20 & 31.09 cluster 0 \\
\hline 21 & 24.96 cluster \\
\hline & 1216.19 cluster \\
\hline & 2611.60 cluster \\
\hline & 70.94 cluster \\
\hline 25 & 768.41 cluster: \\
\hline & 1495.86 cluster \\
\hline 27 & 1400.39 cluster3 \\
\hline 28 & 2252.85 cluster2 2 \\
\hline 29 & 234.70 cluster 0 \\
\hline 30 & 16.03 cluster0 \\
\hline 31 & 170.46 cluster0 \\
\hline 32 & 172.48 cluster \\
\hline 33 & 1348.43 cluster \\
\hline 34 & 285.14 cluster \\
\hline & 46.32 cluster \\
\hline & 280.52 cluster \\
\hline & $432.08 \mathrm{cl}$ \\
\hline 38 & $463.00 \mathrm{cl}$ \\
\hline 39 & $258.62 \mathrm{cl}$ \\
\hline 40 & 309.20 cluster \\
\hline 41 & 306.39 cluster \\
\hline 42 & 450.74 cluster \\
\hline 43 & 145.41 cluster \\
\hline 44 & 523.36 cluster \\
\hline 45 & 207.48 cluster \\
\hline 46 & 34.86 cluster \\
\hline 47 & 78.14 cluster \\
\hline & $66.85 \mathrm{clu}$ \\
\hline & 28.77 \\
\hline 50 & 187.36 \\
\hline 51 & 59.0 \\
\hline 52 & 55.76 \\
\hline 53 & $56.37 \mathrm{cl}$ \\
\hline 54 & 758.59 clus \\
\hline 55 & $197.41 \mathrm{clus}$ \\
\hline 5 & 119.85 clus \\
\hline $57-2>$ & 126.60 clus \\
\hline 58 & 70.44 clus \\
\hline-0 & 46.26 cluster \\
\hline & $15275.48 \mathrm{c}$ \\
\hline & 432. \\
\hline & 1977. \\
\hline & 4054. \\
\hline & $222.94 \mathrm{c}$ \\
\hline
\end{tabular}

\begin{tabular}{|c|c|c|}
\hline \multicolumn{3}{|c|}{ Id Area } \\
\hline & 337.975 & 5 clustero \\
\hline & 186.19 & \\
\hline & 429.83 & 3 clustero \\
\hline & 304.18755 & clustero \\
\hline & 626.14 & 4 clustero \\
\hline & & \\
\hline & 646.575 & \\
\hline & 1400.805 & cluster1 \\
\hline & 505.49 & clustero \\
\hline & 971.60625 & cluster1 \\
\hline & 871.22 & 2 cluster1 \\
\hline & 1186.65 & 5 cluster1 \\
\hline & 1446.375 & cluster1 \\
\hline & 265.58 & 8 clustero \\
\hline & 937.615 & \\
\hline & & \\
\hline & 349.95 & \\
\hline & 314.265 & 5 clustero \\
\hline & 610.66 & 5 clustero \\
\hline & 287.705 & 5 clustero \\
\hline & 134.7 & 7 clustero \\
\hline & 194.995 & 5 clustero \\
\hline & 134.38 & 3 clustero \\
\hline & 2109.325 & 5 cluster 1 \\
\hline & 409.94 & \\
\hline & 85.17 & \\
\hline & 85.405 & cluster0 \\
\hline & 2137.37793 & 3 cluster1 \\
\hline & 22.205 & 5 clustero \\
\hline & 118.87 & clustero \\
\hline & 73.505 & 5 clustero \\
\hline & 772.505 & 5 cluster1 \\
\hline & 494.71839 & clustero \\
\hline & 524.39518 & \\
\hline & & 3 clustero \\
\hline & 1474.895 & 5 cluster1 \\
\hline & $\begin{array}{r}147.095 \\
499.54\end{array}$ & 4 clustero \\
\hline & 707.52 & 2 cluster1 \\
\hline & 234.39 & cluster0 \\
\hline & 393.295 & 5 clustero \\
\hline & & 2 clustero \\
\hline & 290.18 & 8 clustero \\
\hline & 1072.045 & 5 cluster1 \\
\hline & 118.395 & 5 clustero \\
\hline & 288.295 & 5 clustero \\
\hline & 49.655 & 5 clustero \\
\hline & $\begin{array}{r}49.655 \\
340.519955\end{array}$ & $\begin{array}{l}\text { sllustero } \\
\text { clustero }\end{array}$ \\
\hline & 2842.45372 & 2 cluster 1 \\
\hline & $\begin{array}{r}042.35 \\
63.31895\end{array}$ & 5 clustero \\
\hline & 343.39 & clustero \\
\hline & 260.273395 & 5 clustero \\
\hline & 245.465555 & clustero \\
\hline & 389.91 & \\
\hline & 178.46 & \\
\hline & 255.28 & 8 clustero \\
\hline & 1517.455 & 5 cluster1 \\
\hline & 421.453485 & 5 clustero \\
\hline & 142.879785 & 5 clustero \\
\hline & 144.15 & 5 clustero \\
\hline & 125.49 & clustero \\
\hline & 785.505 & 5 cluster1 \\
\hline & 399.07419 & clustero \\
\hline & 133.075 & 5 clustero \\
\hline & 2720.38 & 8 cluster1 \\
\hline & 405.42 & 2 clustero \\
\hline & 186.53 & 3 clustero \\
\hline & 247.475 & 5 clustero \\
\hline & 374.01 & 1 clustero \\
\hline & 1 & 5 clustero \\
\hline & & . \\
\hline & 02803 & clustero \\
\hline
\end{tabular}




\section{Appendix 17: Areas and clusters in Auckland 1882 (chapter 6)}

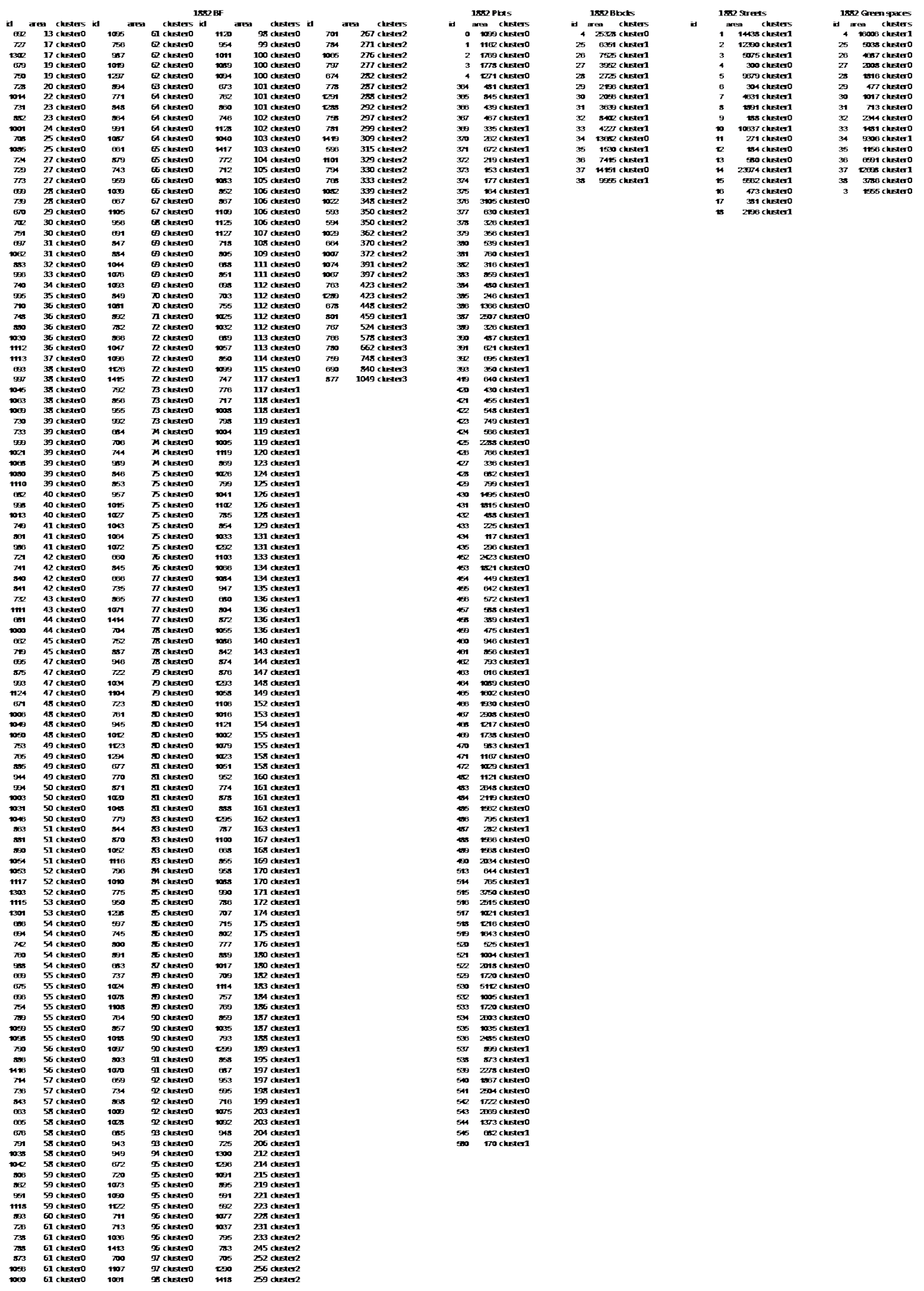




\section{Appendix 18: Areas and clusters in Auckland 1908 (chapter 6)}

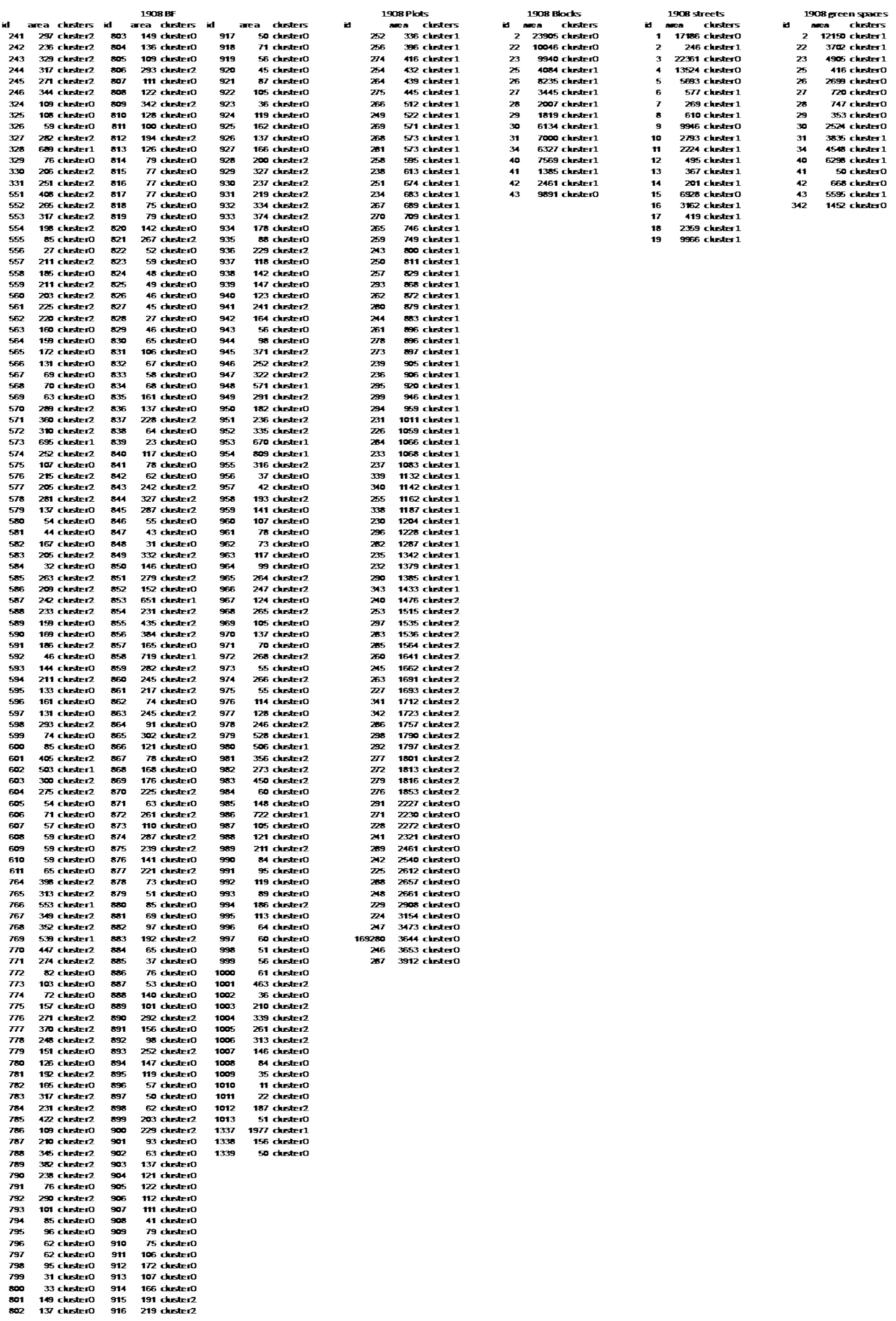


12.Appendix 19: Areas and clusters in Auckland 1931 (chapter 6)

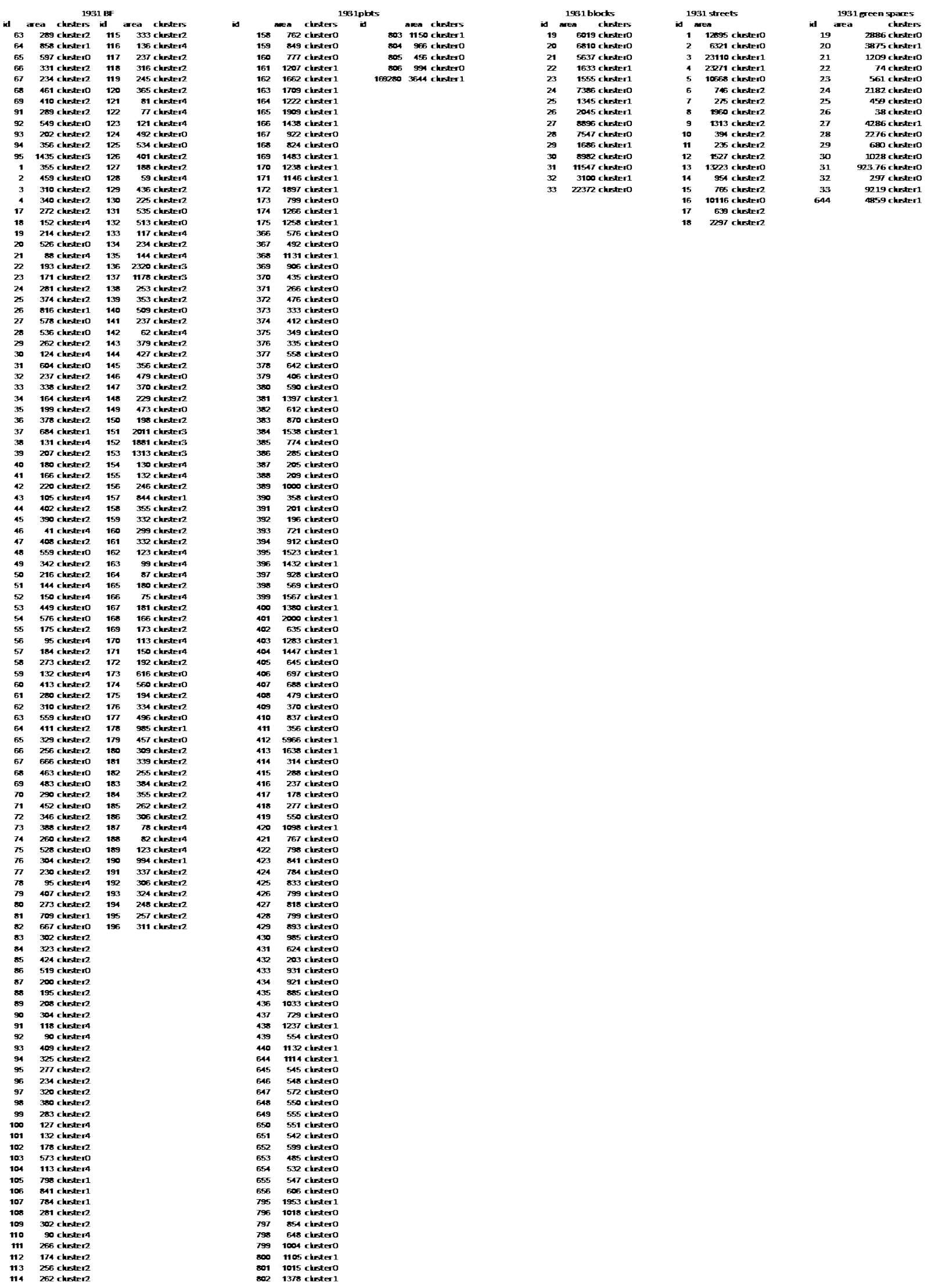


13.Appendix 20: Areas and clusters in Auckland 1966 (chapter 6)
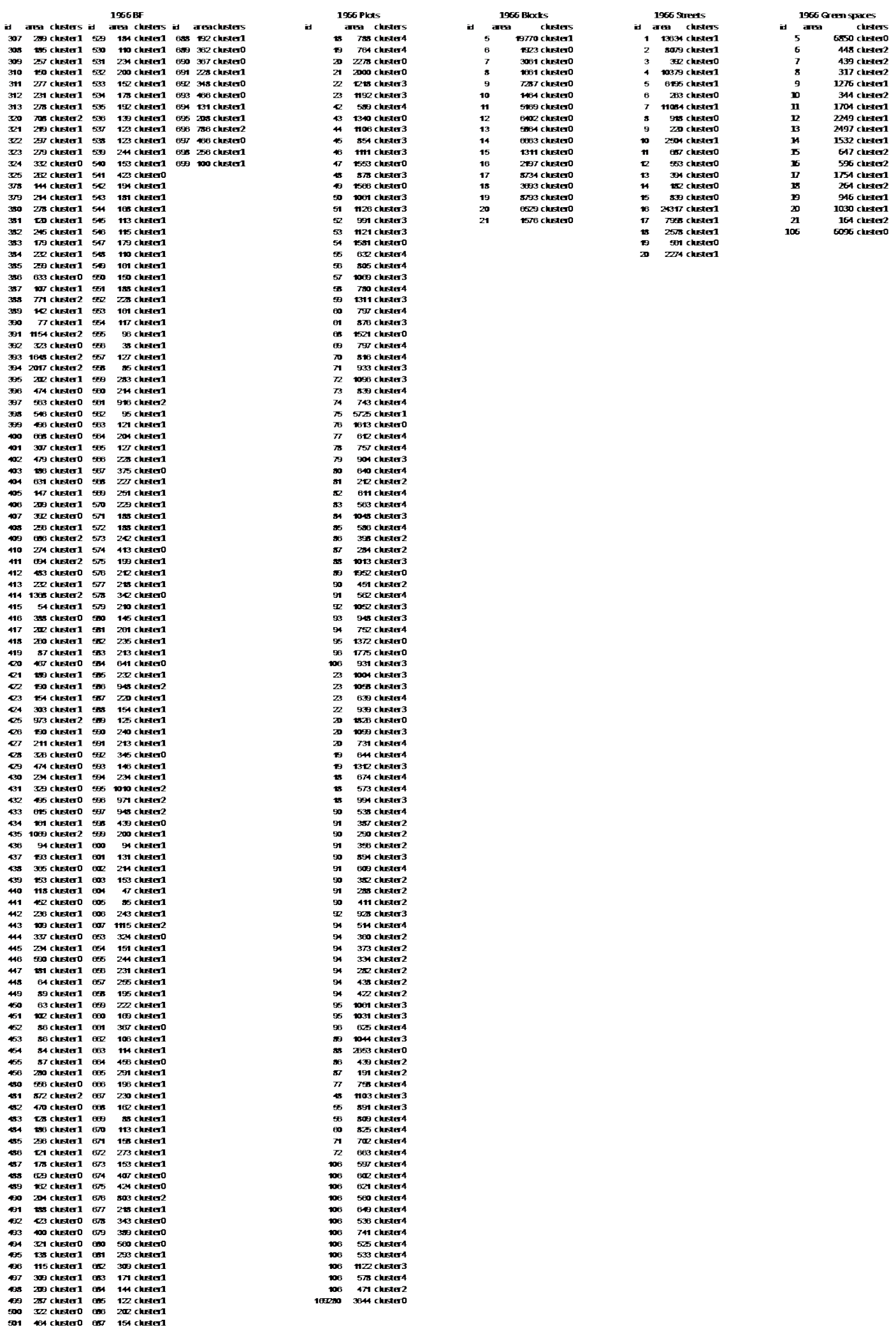

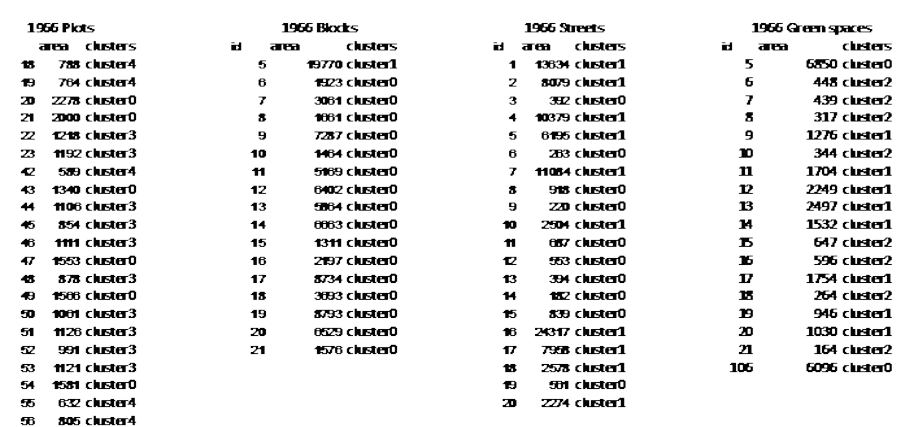


14.Appendix 21: Areas and clusters in Auckland 2008 (chapter 6)
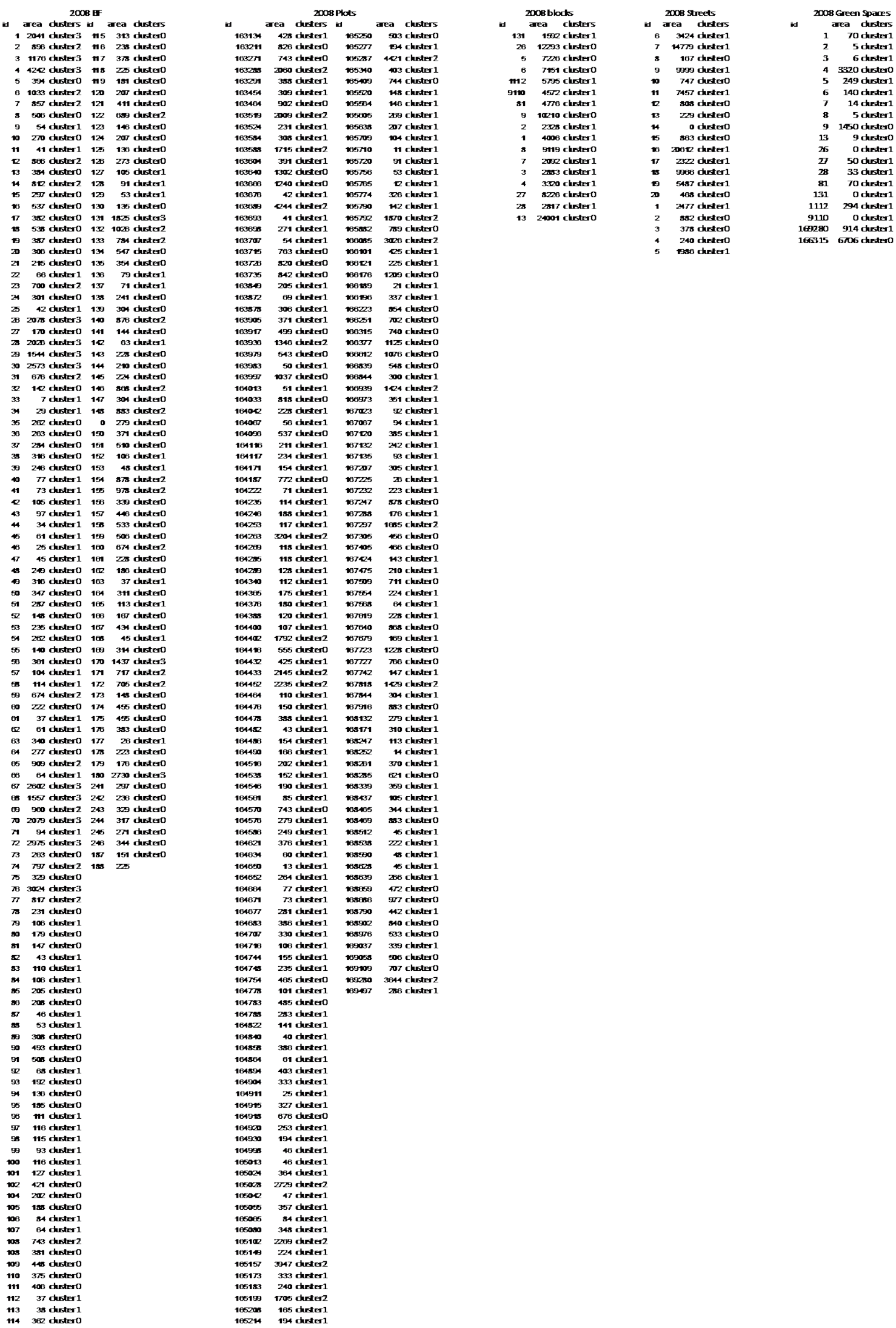